Construction and Regularity of Solutions to Stochastic Partial Differential Equations with Irregular Drift Coefficients

by

Torstein Nilssen

Dissertation presented for the Degree of

Philosophiae Doctor

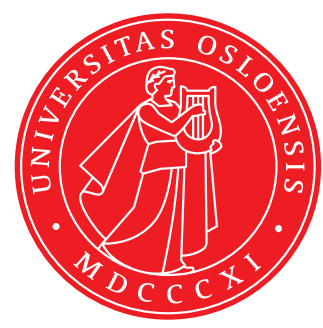

Department of Mathematics

University of Oslo 
(C) Torstein Nilssen, 2013

Series of dissertations submitted to the

Faculty of Mathematics and Natural Sciences, University of Oslo No. 1436

ISSN 1501-7710

All rights reserved. No part of this publication may be

reproduced or transmitted, in any form or by any means, without permission.

Cover: Inger Sandved Anfinsen.

Printed in Norway: AIT Oslo AS.

Produced in co-operation with Akademika Publishing.

The thesis is produced by Akademika Publishing merely in connection with the thesis defence. Kindly direct all inquiries regarding the thesis to the copyright holder or the unit which grants the doctorate. 



\section{Acknowledgments}

This thesis has been funded by Center of Mathematics for Applications (CMA) and the Mathematical Institute both at the University of Oslo. During four years I have had the pleasure of being a part of the Stochastic Analysis group which has given me the opportunity to meet and work with a number of inspiring people.

First and foremost, I would like to thank my primary supervisor, Frank Proske. It is difficult to explain how important his guidance has been for me. For me, there could not be a better supervisor. Our hundreds of talks over hundreds of cups of coffee has always left me motivated and inspired.

I would also like to thank all the people at the CMA for creating such an inspiring environment. In particular I want to thank David Baños, Giulia di Nunno, Sven Haadem, Erlend Storrøsten and Bernt Øksendal for valuable discussions as well as my coauthors Franco Flandoli, Olivier Menokeu Pamen, Thilo Meyer-Brandis, Salah Mohammed and Tusheng Zhang. Also the administrative staff, in particular Biljana Dragisic, Robin Jacobsen and Elisabeth Seland deserves a thank you for making bureaucracy a less painful experience.

Between January and July 2013 I spent my time at Humboldt University of Berlin. I would like to thank Peter Imkeller and his research group for including me in Berlin. In particular, I am grateful to Nicolas Perkowski for interesting discussions.

Finally, I would like to thank the most important person in my life, Ellen, for always being loving and supportive.

Oslo, September 2013

Torstein Nilssen 


\section{Contents}

1 Preface 5

2 A Variational Approach to the construction and Malliavin Differentiability $\begin{array}{lr}\text { of Strong solutions of SDE's } & 9\end{array}$

2.1 Introduction . . . . . . . . . . . . . . . . . . . . . . 10

2.2 Framework . . . . . . . . . . . . . . . . . . . . 11

2.2.1 Basic Facts of Gaussian White Noise Theory . . . . . . . . . . . . 11

2.2.2 Basic elements of Malliavin Calculus . . . . . . . . . . . . . . . . 14

2.3 Main results . . . . . . . . . . . . . . . . . . . . . . . 15

2.4 Applications . . . . . . . . . . . . . . . . . . . . . . . . . 31

2.4.1 Sobolev differentiability of strong solutions of SDE's . . . . . . . . . 31

2.4.2 Stochastic representation of spatial derivatives of solutions to Kol-

mogorov equations . . . . . . . . . . . . . . 34

2.4.3 Well-posedness of stochastic transport equations with singular coefficients 36

2.5 Appendix . . . . . . . . . . . . . . . . . . . 38

3 Sobolev Differentiable Stochastic Flows for SDE's with Singluar Coefficients: Applications to the Transport Equation 43

3.1 Introduction . . . . . . . . . . . . . . . . . . . . . . 44

3.2 Existence of a Sobolev Differentiable Stochastic Flow . . . . . . . . . . . . . . 46

3.3 Application to the Stochastic Transport Equation . . . . . . . . . . . . . . . . 65

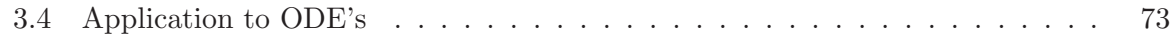

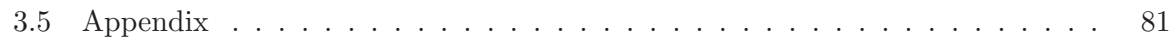

4 Regularity of Strong Solutions of one-dimensional SDE's with discontinu$\begin{array}{lr}\text { ous and unbounded drift } & 86\end{array}$

4.1 Introduction . . . . . . . . . . . . . . . . . . . . . . 87

4.2 Framework . . . . . . . . . . . . . . . . . . . . 88

4.2.1 Basic Facts of Gaussian White Noise Theory . . . . . . . . . . . 88

4.2 .2 Basic elements of Malliavin Calculus . . . . . . . . . . . . . . 90

4.3 Existence and Uniqueness of the Flow . . . . . . . . . . . . . . . . . 90

4.4 Regularity for step functions . . . . . . . . . . . . . . . . . . . . . 103

4.5 Stochastic Transport Equation . . . . . . . . . . . . . . . . . . 106

4.6 Appendix . . . . . . . . . . . . . . . . . . . . . . . . . . . . 107

5 Malliavin differentiability and strong solutions for a class of SDE in Hilbert

$\begin{array}{ll}\text { spaces } & 110\end{array}$

5.1 Introduction . . . . . . . . . . . . . . . . . . . . . . . . . 111

5.1 .1 Notations and assumptions . . . . . . . . . . . . . . . . . . 111

5.2 Idea of the method . . . . . . . . . . . . . . . . . . . . . . . . . . . . . . . . . . . . 112

$5.3 \quad H$-valued Ornstein-Uhlenbeck semigroup . . . . . . . . . . . . . . . . . . . . 113

5.4 Non homogenuous Kolmogorov equation . . . . . . . . . . . . . . . 117 
$5.5 \quad$ Malliavin Differentiability . . . . . . . . . . . . . . . . . . 120

5.5 .1 Strong Uniqueness . . . . . . . . . . . . . . . . . . . 120

5.5 .2 Malliavin Differentiability . . . . . . . . . . . . . . . . . . 123

5.6 Appendix . . . . . . . . . . . . . . . . . . . . . . . . . . . . 148 


\section{Preface}

This thesis consists of four papers produced in the course of the last four years. The purpose of this introduction is to show the connection between the papers and briefly explain the contents without using much technical detail. The papers are presented chronologically with respect to when they were finished.

To put it simple, this thesis deals with the regularization effect of noise on differential equations with 'bad' coefficients.

The discovery of differential equations is arguably the most revolutionary moment in the history of mathematics. A very general, first order Ordinary Differential Equation (ODE) can be written

$$
\frac{d X_{t}}{d t}=b\left(X_{t}\right), X_{0}=x
$$

where $b$ is a given function and we are searching for a differentiable function, $t \mapsto X_{t}$ satisfying the above relation. The most widespread assumption on $b$ one uses to study ODE's is the Lipschitz-continuity, namely

$$
|b(x)-b(y)| \leq L|x-y|
$$

where $L$ is a given constant. Under this assumption it is well known that there exists a unique solution to the equation (1.1). If the Lipschitz condition is not satisfied, existence and/or uniqueness may be violated in (1.1). Consider for example $b(t)=2 \operatorname{sign}(t) \sqrt{|t|}$ and $x=0$. It is then easy to verify that $X_{t}=0$ for all $t$ is a solution. Furthermore $X_{t}^{ \pm}= \pm t^{2}$ also solves the problem. In general, proving existence of a solution to (1.1) is also a challenging problem.

It is an interesting fact that adding noise to the equation has a regularizing effect. Namely, if once replaces (1.1) with its corresponding Stochastic Differential Equation (SDE)

$$
d X_{t}=b\left(X_{t}\right) d t+d B_{t}, X_{0}=x
$$

where $B=\left(B_{t}\right)_{t>0}$ is a Brownian motion, existence and uniqueness holds (in the stochastic sense) as long as $b$ is bounded and measurable. This means that we can allow for discontinuous coefficients, which clearly violates the Lipschitz condition.

This result was first discovered by Zvonkin in [52] for the one-dimensional case and later generalized by Veretennikov in [49] to multiple dimensions. Both of these proofs utilizes the Yamada-Watanabe theorem, i.e. they show that there is strong uniqueness in (1.2) in connection with weak existence which gives the result.

This thesis tries to reach further than existence and uniqueness in the sense of regularity. Indeed, using a different technique, not relying on the Yamada-Watanabe theorem, we have studied the Malliavin differentiability and the stochastic flow of (1.2) when $b$ is bounded and measurable. This is quite counter intuitive, as the common trend in the study of differential equations and dynamical systems is that the solutions 'inherits' the regularity from the driving coefficients. It is important to remark that this technique proves existence and uniqueness as well, i.e. it 'stands alone' in the sense that it does not rely on the results by Zvonkin or Veretennikov. This gives us reason to believe that the method presented in this thesis is in some sense more closely linked to the true nature of these equations.

In more detail, the content of the thesis is as follows: 


\section{Chapter 2}

In this chapter we present the mentioned new method for proving existence and uniqueness of a solution to (1.2) when $b$ is just bounded and measurable. As a by-product we get that the solution is Malliavin differentiable.

More precisely, we approximate a given function $b$ by a sequence of smooth functions $\left\{b_{n}\right\}$, uniformly bounded in $L^{\infty}$. It is then well known that the corresponding solution, $X^{n}=\left(X_{t}^{n}\right)_{t \geq 0}$ to (1.2) is Malliavin differentiable and its Malliavin derivative satisfies

$$
D_{s} X_{t}^{n}=I_{d \times d}+\int_{s}^{t} b_{n}^{\prime}\left(u, X_{u}^{n}\right) D_{s} X_{u}^{n} d u
$$

which can be regarded as linear ODE with a random coefficient. We then use Picard iteration to write

$$
D_{s} X_{t}^{n}=I_{d \times d}+\sum_{k \geq 1} \int_{s<u_{1}<\ldots u_{k}<t} b_{n}^{\prime}\left(u_{1}, X_{u_{1}}\right) \ldots b_{n}^{\prime}\left(u_{k}, X_{u_{k}}\right) d u_{1} \ldots d u_{k} .
$$

The trick is then to find a bound on $D_{s} X_{t}^{n}$ which does not depend on the size of $b_{n}^{\prime}$, but rather $\left\|b_{n}\right\|_{L^{\infty}}$. Using a compactness criterion in [6] one can show convergence of $\left\{X_{t}^{n}\right\}$ in the strong topology of $L^{2}(\Omega)$. Then, using an explicit representation formula for the solution developed in [3] we can show that $X_{t}^{n}$ is converging to a process $X=\left(X_{t}\right)_{t \geq 0}$ which solves (1.2). Since the norm of $D_{s} X_{t}^{n}$ is uniformly bounded, this is enough to guarantee Malliavin differentiability of $X_{t}$.

\section{Chapter 3}

In this chapter we consider the same equation (1.2) with a bounded and measurable $b$, but to emphasize its dependence on the initial condition, $x$, we write $X_{t}^{x}$. In this chapter we show that the mapping $x \mapsto X_{t}^{x}$ is weakly differentiable.

Using the same approximation of $b$ by $\left\{b_{n}\right\}_{n \geq 1}$, we note that the corresponding solution $X_{t}^{n, x}$ is differentiable in $x$ and that the derivative satisfies

$$
\frac{\partial}{\partial x} X_{t}^{n, x}=I_{d \times d}+\int_{0}^{t} b_{n}^{\prime}\left(u, X_{u}^{n}\right) \frac{\partial}{\partial x} X_{u}^{n, x} d u
$$

which is the same equation as (1.3) when $s=0$.

Similarly as in Chapter 2 we find a bound of the norm of $\frac{\partial}{\partial x} X_{t}^{n, x}$ depending only on $\left\|b_{n}\right\|_{L^{\infty}}$. Using weak compactness in connection with the strong compactness from Chapter 2 we can conclude that the mapping $x \mapsto X_{t}^{x}$ is weakly differentiable, almost surely.

This fact is applied to study the Stochastic Transport Equation,

$$
\begin{aligned}
\partial_{t} u(t, x)+b(t, x) \partial_{x} u(t, x)+\partial_{x} u(t, x) \circ d B_{t} & =0 \\
u(0, x) & =u_{0}(x) .
\end{aligned}
$$

Notice that, since $b$ is not differentiable, the usual trick of integrating against a testfunction and using integration by parts does not make sense for the product $b(t, x) \partial_{x} u(t, x)$. This chapter shows, however, that the solution is weakly differentiable so that one does not need to use integration by parts on this term, and this leads to a meaningful definition of a weak solution even for discontinuous $b$. Indeed, the solution to (1.5) is given by $u(t, x)=$ $u_{0}\left(\phi_{t}^{-1}(x)\right)$ where $\phi_{t}(x)$ is the flow map associated with (1.2). This relation is easily checked 
by using the usual method of characteristic for (1.5) in connection with the Itô-Ventzell formula.

\section{Chapter 4}

This chapter deals with the special case of one-dimensional equation and autonomous $b$. However, it opens up for the study of an unbounded coefficient, where existence and uniqueness is not covered by Zvonking or Veretennikov.

More precisely, we consider a sub-linear coefficient, $|b(x)| \leq C(1+|x|)$, which is enough to guarantee weak existence of a solution which does not have a finite blow-up time, see [2].

The advantage of one-dimension lies in the fact that in this case (1.3) can be solved explicitly by

$$
D_{s} X_{t}^{n, x}=\exp \left\{\int_{s}^{t} b_{n}^{\prime}\left(X_{u}^{n, x}\right) d u\right\} .
$$

The trick is this time is to use Itô's formula to get rid of the derivative of $b_{n}$. Namely, let $\tilde{b}_{n}$ be an anti-derivative of $b_{n}$ and use Itô's formula to write

$$
\tilde{b}_{n}\left(X_{t}^{n, x}\right)=\tilde{b}_{n}\left(X_{s}^{n, x}\right)+\int_{s}^{t} b_{n}\left(X_{u}^{n, x}\right) d X_{u}^{n, x}+\frac{1}{2} \int_{s}^{t} b_{n}^{\prime}\left(X_{u}^{n, x}\right) d u .
$$

We can then find an explicit formula for the spatial derivative of the flow of (1.2), which does not use the derivative of the coefficient but rather a local time integral:

$$
\frac{\partial}{\partial x} X_{t}^{x}=\exp \left\{-\int_{\mathbb{R}} b(y) d L_{t}^{y}\left(X_{\cdot}^{x}\right)\right\} .
$$

Using this representation it follows in a straightforward (but technical) application of Kolmogorov's continuity lemma that we get a continuous derivative of $x \mapsto X_{t}^{x}$ when $b$ is a step function.

\section{Chapter 5}

In the final chapter we consider a Hilbert-space valued SDE,

$$
d X_{t}=\left(A X_{t}+B\left(t, X_{t}\right)\right) d t+\sqrt{Q} d W_{t}, X_{0}=x \in H
$$

where the coefficient $B$ is Hölder continuous, i.e.

$$
|B(t, x)-B(t, y)| \leq C|x-y|^{\alpha}
$$

for some $\alpha \in(0,1)$. Here, $A$ is a linear (discontinuous) operator, $W$ is a cylindrical Brownian motion on $H$ and $Q$ a non-negative selfadjoint bounded operator on $H$. We show existence and uniqueness of a solution to this equation which is Malliavin differentiable. Notice that the assumption of Hölder continuity is substantially weaker than Lipschitz continuity.

The proof of this assertion combines the method introduced in Chapter 2 with estimates on the solution of the following infinite-dimensional PDE for every $n \in \mathbb{N}$

$$
\begin{aligned}
\frac{\partial U_{n}}{\partial t}+\frac{1}{2} \operatorname{Tr}\left(D^{2} U_{n} Q\right)+\left\langle A x, D U_{n}\right\rangle+\left\langle B, D U_{n}\right\rangle & =B_{n} \\
U_{n}(T, x) & =0
\end{aligned}
$$


where $B_{n}$ is the $n$-th component of $B$. The presense of $D^{2}$ has a regularizing effect in the sense that if $B$ is Hölder continuous, the solution to (1.7) is twice differentiable. Letting $U(t, x)=\sum_{n \in \mathbb{N}} U_{n}(t, x) e_{n}$ we can apply Itô's formula to $U$ and get

$$
d U\left(t, X_{t}\right)=B\left(t, X_{t}\right) d t+D U\left(t, X_{t}\right) \sqrt{Q} d W_{t} .
$$

Then we can rewrite (1.6) as

$$
\begin{gathered}
X_{t}=e^{t A}(x-U(0, x))+U\left(t, X_{t}\right)+\int_{0}^{t} A e^{(t-s) A} U\left(s, X_{s}\right) d s \\
\quad+\int_{0}^{t} e^{(t-s) A} Q^{1 / 2} d W_{s}-\int_{0}^{t} e^{(t-s) A} D U\left(s, X_{s}\right) Q^{1 / 2} d W_{s} .
\end{gathered}
$$

In this new formulation all the right hand side terms have good regularity properties.

\section{References}

[1] G. Da Prato, P. Malliavin, D. Nualart, Compact families of Wiener functionals. C. R. Acad. Sci. Paris, Sr. I 315 (1992), 1287-1291.

[2] Karatzas I., Shreve, S.E.: Brownian Motion and Stochastic Calculus, Springer-Verlag (1988).

[3] A. Lanconelli, F. Proske, On explicit strong solutions of Itô-SDE's and the Donsker delta function of a diffusion. Infin. Dimen. Anal. Quant. Prob. related Topics, 7 (3) (2004).

[4] A.Y. Veretennikov, On the strong solutions of stochastic differential equations. Theory Probab. Appl. 24 (1979), 354-366.

[5] A.K. Zvonkin, A transformation of the state space of a diffusion process that removes the drift. Math.USSR (Sbornik) 22 (1974), 129-149. 


\title{
2 A Variational Approach to the construction and Malliavin Differentiability of Strong solutions of SDE's
}

\author{
Olivier Menoukeu-Pamen, Thilo Meyer-Bandis, Torstein Nilssen, \\ Frank Proske and Tusheng Zang \\ Published in Mathematische Annalen, \\ Vol. 357, Issue 2, pp 761-799, 2013
}

\begin{abstract}
In this article we develop a new approach to construct solutions of stochastic equations with merely measurable drift coefficients. We aim at demonstrating the principles of our technique by analyzing strong solutions of stochastic differential equations driven by Brownian motion. An important and rather surprising consequence of our method which is based on Malliavin calculus is that the solutions derived by A. Y. Veretennikov [49] for Brownian motion with bounded and measurable drift in $\mathbb{R}^{d}$ are Malliavin differentiable. Further, a strength of our approach, which doesn't rely on a pathwise uniqueness argument, is that it can be transferred and applied to the analysis of various other types of stochastic equations: We prove Sobolev differentiability of stochastic differential equations in their initial conditions. The latter result is used to derive a Bismut-Elworthy-Li formula [10] for spatial derivatives of solutions to the Kolmogorov equation under weak conditions on the drift coefficient. Another application of our technique is the construction of unique solutions of the stochastic transport equation with irregular drift coefficients. Moreover, it is conceivable that our approach is also applicable to the construction of solutions of stochastic evolution equations on Hilbert spaces.
\end{abstract}




\section{$2.1 \quad$ Introduction}

In this paper we are mainly interested to study the following stochastic differential equation (SDE) given by

$$
d X_{t}=b\left(t, X_{t}\right) d t+d B_{t}, \quad 0 \leq t \leq T, \quad X_{0}=x \in \mathbb{R}^{d},
$$

where the drift coefficient $b:[0, T] \times \mathbb{R}^{d} \longrightarrow \mathbb{R}^{d}$ is a Borel measurable function and $B_{t}$ is a $d$-dimensional Brownian motion on a probability space $(\Omega, \mathcal{F}, \pi)$. We denote by $\mathcal{F}_{t}$ the augmented filtration generated by $B_{t}$.

If $b$ in (2.1) is of linear growth and (globally) fulfills a Lipschitz condition it is well known that there exists a unique global strong solution to the SDE (2.1). More precisely, there exists a continuous $\mathcal{F}_{t}$-adapted process $X_{t}$ solving (2.1) such that

$$
E\left[\int_{0}^{T} X_{t}^{2} d t\right]<\infty .
$$

Important applications, however, of SDE's of the type (2.1) to physics or stochastic control theory show that Lipschitz continuity imposed on the drift coefficient $b$ is a rather severe restriction. For example, in statistical mechanics, where one is interested in solutions of (2.1) as functionals of the driving noise (i.e. strong solutions) to model interacting infinite particle systems, the drift $b$ is typically discontinuous or singular. See e.g. [21] and the references therein.

Strong solutions of SDE's with non-Lipschitz coefficients have been investigated by many authors in the past decades. To begin with we mention the work of Zvonkin [52], where the author obtains unique strong solutions of (2.1) in the one-dimensional case, when $b$ is merely bounded and measurable. The latter result can be regarded as a milestone in the theory of SDE's. Subsequently, this result was generalized by Veretennikov [49] to the multidimensional case. The tools used by these authors to derive strong solutions are based on estimates of solutions of parabolic partial differential equations and a pathwise uniqueness argument.

Other important and more recent results in this direction based on a pathwise uniqueness argument (in connection with other techniques due to Portenko [36] or the Skorohod embedding) can be e.g. found in Krylov, Röckner [21], Gyöngy, Krylov [16] or Gyöngy, Martínez [17]. We also refer to [11], where the authors employ a modified version of Gronwall's Lemma. In this context we shall also point out the paper of Davie [7], who even establishes uniqueness of strong solutions of (2.1) for almost all Brownian paths in the case of bounded and measurable drift coefficients.

In this paper we further develop the new approach devised in [31] to construct strong solutions of SDE's with irregular drift coefficients which additionally yields the important insight that these solutions are Malliavin differentiable. See also [29] and [38]. More precisely, we derive the results in [31] without assuming a certain symmetry condition [31, Definition 3] on the drift $b$ in (2.1), which severely restricts the class of SDE's to be studied. In particular, one of our main results is the extension of [31, Theorem 4] on the Malliavin differentiability of solutions of (2.1) for merely bounded Borel functions $b$ from the one-dimensional to the multidimensional case.

Our approach is mainly based on Malliavin calculus. To be more precise, our technique relies on a compactness criterion based on Malliavin calculus and an approximation argument for certain generalized processes in the Hida distribution space which we directly verify to be strong solutions of (2.1). We remark that our construction method is different from the above mentioned authors' ones. The technique proposed in this paper is not based on a pathwise 
uniqueness argument (or the Yamada-Watanabe theorem). In fact we tackle the construction problem from the "opposite" direction and prove that strong existence in connection with uniqueness in law of solutions of SDE's enforces strong uniqueness.

The additional information that strong solutions of SDE's with merely measurable drift coefficients are Malliavin differentiable has important and interesting implications. Further, one major strength of our approach is that it exhibits great flexibility to be applied and generalized to the analysis of various important aspects of solutions of a broader range of stochastic equations with irregular coefficients besides finite dimensional SDE's. In the last section of this paper we illustrate this by first showing how our techniques imply Sobolev differentiability of the strong solution $X_{t}$ of SDE (2.1) in the initial condition $x$. Together with the Malliavin differentiability of $X_{t}$ this is then used to derive a useful stochastic representation of spatial derivatives of solutions to the Kolmogorov equation - known as Bismut-Elworthy$\mathrm{Li}$ formula - which does not involve derivatives of the initial condition of the Kolmogorov equation. Secondly, we present the applicability of our techniques and results to the problem of well-posedness of stochastic transport equations with singular coefficients (see also [32]).

The paper is organized as follows: In Section 2.2 we recall basic concepts of Malliavin calculus and Gaussian white noise theory. Section 2.3 is devoted to the study of the SDE (2.1). The main results of the paper are Theorem 2.3, Lemma 2.5, Corollary 2.6, and Theorem 2.17. Section 2.4 concludes by considering the above mentioned applications of techniques and results of the previous sections.

\section{$2.2 \quad$ Framework}

In this section we recall some facts from Gaussian white noise analysis and Malliavin calculus, which we aim at employing in Section 2.3 to construct strong solutions of SDE's. See [8, 35, 23] for more information on white noise theory. As for Malliavin calculus the reader is referred to $[34,25,26,8]$.

\subsubsection{Basic Facts of Gaussian White Noise Theory}

A building block of our proof for the constuction of strong solutions (see Section 2.3) is based on a generalized stochastic process in the Hida distribution space which we verify to be a SDE solution. In the following, we shall give the definition of this space which goes back to T. Hida (see [8]).

From now on we fix a time horizon $0<T<\infty$. Consider a (positive) self-adjoint operator $A$ on $L^{2}([0, T])$ with $\operatorname{Spec}(A)>1$. Let us require that $A^{-r}$ is of Hilbert-Schmidt type for some $r>0$. Denote by $\left\{e_{j}\right\}_{j \geq 0}$ a complete orthonormal basis of $L^{2}([0, T])$ in $\operatorname{Dom}(A)$ and let $\lambda_{j}>0, j \geq 0$ be the eigenvalues of $A$ such that

$$
1<\lambda_{0} \leq \lambda_{1} \leq \ldots \longrightarrow \infty .
$$

Let us assume that each basis element $e_{j}$ is a continuous function on $[0, T]$. Further let $O_{\lambda}, \lambda \in \Gamma$, be an open covering of $[0, T]$ such that

$$
\sup _{j \geq 0} \lambda_{j}^{-\alpha(\lambda)} \sup _{t \in O_{\lambda}}\left|e_{j}(t)\right|<\infty
$$

for $\alpha(\lambda) \geq 0$. 
In what follows let $\mathcal{S}([0, T])$ denote the standard countably Hilbertian space constructed from $\left(L^{2}([0, T]), A\right)$. See [35]. Then $\mathcal{S}([0, T])$ is a nuclear subspace of $L^{2}([0, T])$. We denote by $\mathcal{S}^{\prime}([0, T])$ the corresponding conuclear space, that is the topological dual of $\mathcal{S}([0, T])$. Then the Bochner-Minlos theorem provides the existence of a unique probability measure $\pi$ on $\mathcal{B}\left(\mathcal{S}^{\prime}([0, T])\right)$ (Borel $\sigma$-algebra of $\left.\mathcal{S}^{\prime}([0, T])\right)$ such that

$$
\int_{\mathcal{S}^{\prime}([0, T])} e^{i\langle\omega, \phi\rangle} \pi(d \omega)=e^{-\frac{1}{2}\|\phi\|_{L^{2}([0, T])}^{2}}
$$

holds for all $\phi \in \mathcal{S}([0, T])$, where $\langle\omega, \phi\rangle$ is the action of $\omega \in \mathcal{S}^{\prime}([0, T])$ on $\phi \in \mathcal{S}([0, T])$. Set

$$
\Omega_{i}=\mathcal{S}^{\prime}([0, T]), \quad \mathcal{F}_{i}=\mathcal{B}\left(\mathcal{S}^{\prime}([0, T])\right), \quad \mu_{i}=\pi,
$$

for $i=1, \ldots, d$. Then the product measure

$$
\mu=\underset{i=1}{\stackrel{d}{\times}} \mu_{i}
$$

on the measurable space

$$
(\Omega, \mathcal{F}):=\left(\prod_{i=1}^{d} \Omega_{i}, \stackrel{d}{\otimes} \mathcal{F}_{i=1}\right)
$$

is referred to as $d$-dimensional white noise probability measure.

Consider the Doleans-Dade exponential

$$
\widetilde{e}(\phi, \omega)=\exp \left(\langle\omega, \phi\rangle-\frac{1}{2}\|\phi\|_{L^{2}\left([0, T] ; \mathbb{R}^{d}\right)}^{2}\right),
$$

for $\omega=\left(\omega_{1}, \ldots, \omega_{d}\right) \in\left(\mathcal{S}^{\prime}([0, T])\right)^{d}$ and $\phi=\left(\phi^{(1)}, \ldots, \phi^{(d)}\right) \in(\mathcal{S}([0, T]))^{d}$, where $\langle\omega, \phi\rangle:=$ $\sum_{i=1}^{d}\left\langle\omega_{i}, \phi_{i}\right\rangle$.

In the following let $\left((\mathcal{S}([0, T]))^{d}\right)^{\widehat{\otimes} n}$ be the $n$-th completed symmetric tensor product of $(\mathcal{S}([0, T]))^{d}$ with itself. One verifies that $\widetilde{e}(\phi, \omega)$ is holomorphic in $\phi$ around zero. Hence there exist generalized Hermite polynomials $H_{n}(\omega) \in\left(\left((\mathcal{S}([0, T]))^{d}\right)^{\widehat{\otimes} n}\right)^{\prime}$ such that

$$
\widetilde{e}(\phi, \omega)=\sum_{n \geq 0} \frac{1}{n !}\left\langle H_{n}(\omega), \phi^{\otimes n}\right\rangle
$$

for $\phi$ in a certain neighbourhood of zero in $(\mathcal{S}([0, T]))^{d}$. It can be shown that

$$
\left\{\left\langle H_{n}(\omega), \phi^{(n)}\right\rangle: \phi^{(n)} \in\left((\mathcal{S}([0, T]))^{d}\right)^{\widehat{\otimes} n}, n \in \mathbb{N}_{0}\right\}
$$

is a total set of $L^{2}(\mu)$. Further one finds that the orthogonality relation

$$
\int_{\mathcal{S}^{\prime}}\left\langle H_{n}(\omega), \phi^{(n)}\right\rangle\left\langle H_{m}(\omega), \psi^{(m)}\right\rangle \mu(d \omega)=\delta_{n, m} n !\left(\phi^{(n)}, \psi^{(n)}\right)_{L^{2}\left([0, T]^{n} ;\left(\mathbb{R}^{d}\right)^{\otimes n}\right)}
$$

is valid for all $n, m \in \mathbb{N}_{0}, \phi^{(n)} \in\left((\mathcal{S}([0, T]))^{d}\right)^{\widehat{\otimes} n}, \psi^{(m)} \in\left((\mathcal{S}([0, T]))^{d}\right)^{\widehat{\otimes} m}$ where

$$
\delta_{n, m}=\left\{\begin{array}{cc}
1 & \text { if } n=m \\
0 & \text { else }
\end{array} .\right.
$$


Define $\widehat{L}^{2}\left([0, T]^{n} ;\left(\mathbb{R}^{d}\right)^{\otimes n}\right)$ as the space of square integrable symmetric functions $f\left(x_{1}, \ldots, x_{n}\right)$ with values in $\left(\mathbb{R}^{d}\right)^{\otimes n}$. Then the orthogonality relation (2.6) implies that the mappings

$$
\phi^{(n)} \longmapsto\left\langle H_{n}(\omega), \phi^{(n)}\right\rangle
$$

from $\left(\mathcal{S}([0, T])^{d}\right)^{\widehat{\otimes} n}$ to $L^{2}(\mu)$ possess unique continuous extensions

$$
I_{n}: \widehat{L}^{2}\left([0, T]^{n} ;\left(\mathbb{R}^{d}\right)^{\otimes n}\right) \longrightarrow L^{2}(\mu)
$$

for all $n \in \mathbb{N}$. We remark that $I_{n}\left(\phi^{(n)}\right)$ can be viewed as an $n$-fold iterated Itô integral of $\phi^{(n)} \in \widehat{L}^{2}\left([0, T]^{n} ;\left(\mathbb{R}^{d}\right)^{\otimes n}\right)$ with respect to a $d$-dimensional Wiener process

$$
B_{t}=\left(B_{t}^{(1)}, \ldots, B_{t}^{(d)}\right)
$$

on the white noise space

$$
(\Omega, \mathcal{F}, \mu)
$$

It turns out that square integrable functionals of $B_{t}$ admit a Wiener-Itô chaos representation which can be regarded as an infinite-dimensional Taylor expansion, that is

$$
L^{2}(\mu)=\bigoplus_{n \geq 0} I_{n}\left(\widehat{L}^{2}\left([0, T]^{n} ;\left(\mathbb{R}^{d}\right)^{\otimes n}\right)\right)
$$

We construct the Hida stochastic test function and distribution space by using the WienerItô chaos decomposition (2.9). For this purpose let

$$
A^{d}:=(A, \ldots, A),
$$

where $A$ was the operator introduced in the beginning of the section. We define the Hida stochastic test function space $(\mathcal{S})$ via a second quantization argument, that is we introduce $(\mathcal{S})$ as the space of all $f=\sum_{n \geq 0}\left\langle H_{n}(\cdot), \phi^{(n)}\right\rangle \in L^{2}(\mu)$ such that

$$
\|f\|_{0, p}^{2}:=\sum_{n \geq 0} n !\left\|\left(\left(A^{d}\right)^{\otimes n}\right)^{p} \phi^{(n)}\right\|_{L^{2}\left([0, T]^{n} ;\left(\mathbb{R}^{d}\right)^{\otimes n}\right)}^{2}<\infty
$$

for all $p \geq 0$. It turns out that the space $(\mathcal{S})$ is a nuclear Fréchet algebra with respect to multiplication of functions and its topology is given by the seminorms $\|\cdot\|_{0, p}, p \geq 0$. Further one observes that

$$
\widetilde{e}(\phi, \omega) \in(\mathcal{S})
$$

for all $\phi \in(\mathcal{S}([0, T]))^{d}$.

In the sequel we refer to the topological dual of $(\mathcal{S})$ as Hida stochastic distribution space $(\mathcal{S})^{*}$. Thus we have constructed the Gel'fand triple

$$
(\mathcal{S}) \hookrightarrow L^{2}(\mu) \hookrightarrow(\mathcal{S})^{*} .
$$

The Hida distribution space $(\mathcal{S})^{*}$ exhibits the crucial property that it contains the white noise of the coordinates of the $d$-dimensional Wiener process $B_{t}$, that is the time derivatives

$$
W_{t}^{i}:=\frac{d}{d t} B_{t}^{i}, i=1, \ldots, d,
$$


belong to $(\mathcal{S})^{*}$.

We shall also recall the definition of the $S$-transform which is an important tool to characterize elements of the Hida test function and distribution space. See [37]. The $S$-transform of a $\Phi \in(\mathcal{S})^{*}$, denoted by $S(\Phi)$, is defined by the dual pairing

$$
S(\Phi)(\phi)=\langle\Phi, \widetilde{e}(\phi, \omega)\rangle
$$

for $\phi \in\left(\mathcal{S}_{\mathbb{C}}([0, T])\right)^{d}$. Here $\mathcal{S}_{\mathbb{C}}([0, T])$ the complexification of $\mathcal{S}([0, T])$. We mention that the $S$-transform is a monomorphism from $(\mathcal{S})^{*}$ to $\mathbb{C}$. In particular, if

$$
S(\Phi)=S(\Psi) \text { for } \Phi, \Psi \in(\mathcal{S})^{*}
$$

then

$$
\Phi=\Psi .
$$

One checks that

$$
S\left(W_{t}^{i}\right)(\phi)=\phi^{i}(t), i=1, \ldots, d
$$

for $\phi=\left(\phi^{(1)}, \ldots, \phi^{(d)}\right) \in\left(\mathcal{S}_{\mathbb{C}}([0, T])\right)^{d}$.

Finally, we need the important concept of the Wick or Wick-Grassmann product, which we want to use in Section 2.3 to represent solutions of SDE's. The Wick product can be regarded as a tensor algebra multiplication on the Fock space and can be defined as follows: The Wick product of two distributions $\Phi, \Psi \in(\mathcal{S})^{*}$, denoted by $\Phi \diamond \Psi$, is the unique element in $(\mathcal{S})^{*}$ such that

$$
S(\Phi \diamond \Psi)(\phi)=S(\Phi)(\phi) S(\Psi)(\phi)
$$

for all $\phi \in\left(\mathcal{S}_{\mathbb{C}}([0, T])\right)^{d}$. As an example we find that

$$
\left\langle H_{n}(\omega), \phi^{(n)}\right\rangle \diamond\left\langle H_{m}(\omega), \psi^{(m)}\right\rangle=\left\langle H_{n+m}(\omega), \phi^{(n)} \widehat{\otimes} \psi^{(m)}\right\rangle
$$

for $\phi^{(n)} \in\left((\mathcal{S}([0, T]))^{d}\right)^{\widehat{\otimes} n}$ and $\psi^{(m)} \in\left((\mathcal{S}([0, T]))^{d}\right)^{\widehat{\otimes} m}$. The latter in connection with $(2.4)$ shows that

$$
\widetilde{e}(\phi, \omega)=\exp ^{\diamond}(\langle\omega, \phi\rangle)
$$

for $\phi \in(\mathcal{S}([0, T]))^{d}$. Here the Wick exponential $\exp ^{\diamond}(X)$ of a $X \in(\mathcal{S})^{*}$ is defined as

$$
\exp ^{\diamond}(X)=\sum_{n \geq 0} \frac{1}{n !} X^{\diamond n}
$$

where $X^{\diamond n}=X \diamond \ldots \diamond X$, if the sum on the right hand side converges in $(\mathcal{S})^{*}$.

\subsubsection{Basic elements of Malliavin Calculus}

In this Section we briefly elaborate a framework for Malliavin calculus.

Without loss of generality we consider the case $d=1$. Let $F \in L^{2}(\mu)$. Then it follows from (2.9) that

$$
F=\sum_{n \geq 0}\left\langle H_{n}(\cdot), \phi^{(n)}\right\rangle
$$

for unique $\phi^{(n)} \in \widehat{L}^{2}\left([0, T]^{n}\right)$. Assume that

$$
\sum_{n \geq 1} n n !\left\|\phi^{(n)}\right\|_{L^{2}\left([0, T]^{n}\right)}^{2}<\infty .
$$


Then the Malliavin derivative $D_{t}$ of $F$ in the direction of $B_{t}$ is defined by

$$
D_{t} F=\sum_{n \geq 1} n\left\langle H_{n-1}(\cdot), \phi^{(n)}(\cdot, t)\right\rangle .
$$

We introduce the stochastic Sobolev space $\mathbb{D}_{1,2}$ as the space of all $F \in L^{2}(\mu)$ such that $(2.21)$ is fulfilled. The Malliavin derivative $D$. is a linear operator from $\mathbb{D}_{1,2}$ to $L^{2}(\lambda \times \mu)$, where $\lambda$ denotes the Lebesgue measure. We mention that $\mathbb{D}_{1,2}$ is a Hilbert space with the norm $\|\cdot\|_{1,2}$ given by

$$
\|F\|_{1,2}^{2}:=\|F\|_{L^{2}(\mu)}^{2}+\|D \cdot F\|_{L^{2}([0, T] \times \Omega, \lambda \times \mu)}^{2} .
$$

We obtain the following chain of continuous inclusions:

$$
(\mathcal{S}) \hookrightarrow \mathbb{D}_{1,2} \hookrightarrow L^{2}(\mu) \hookrightarrow \mathbb{D}_{-1,2} \hookrightarrow(\mathcal{S})^{*},
$$

where $\mathbb{D}_{-1,2}$ is the dual of $\mathbb{D}_{1,2}$.

\subsection{Main results}

In this section, we want to further develop the ideas introduced in [31] to derive Malliavin differentiable strong solutions of stochastic differential equations with discontinuous coefficients. More precisely, we aim at analyzing the SDE's of the form

$$
d X_{t}=b\left(t, X_{t}\right) d t+d B_{t}, 0 \leq t \leq 1, \quad X_{0}=x \in \mathbb{R}^{d},
$$

where the drift coefficient $b:[0, T] \times \mathbb{R}^{d} \longrightarrow \mathbb{R}^{d}$ is a Borel measurable function and $B_{t}$ is a $d$-dimensional Brownian motion with respect to the stochastic basis

$$
(\Omega, \mathcal{F}, \mu),\left\{\mathcal{F}_{t}\right\}_{0 \leq t \leq T}
$$

for the $\mu$-augmented filtration $\left\{\mathcal{F}_{t}\right\}_{0 \leq t \leq T}$ generated by $B_{t}$. At the end of this section we shall also apply our technique to equations with more general diffusions coefficients (Theorem $3.16)$.

Our method to construct strong solution is actually motivated by the following observation in [24] and [29] (see also [30]).

Proposition 2.1 Suppose that the drift coefficient $b:[0, T] \times \mathbb{R}^{d} \longrightarrow \mathbb{R}^{d}$ in (2.25) is bounded and Lipschitz continuous. Then the unique strong solution $X_{t}=\left(X_{t}^{1}, \ldots, X_{t}^{d}\right)$ of (2.25) allows for the explicit representation

$$
\varphi\left(t, X_{t}^{i}(\omega)\right)=E_{\widetilde{\mu}}\left[\varphi\left(t, \widetilde{B}_{t}^{i}(\widetilde{\omega})\right) \mathcal{E}_{T}^{\diamond}(b)\right]
$$

for all $\varphi:[0, T] \times \mathbb{R} \longrightarrow \mathbb{R}$ such that $\varphi\left(t, B_{t}^{i}\right) \in L^{2}(\mu)$ for all $0 \leq t \leq T, i=1, \ldots, d$,. The object $\mathcal{E}_{T}^{\diamond}(b)$ is given by

$$
\begin{aligned}
\mathcal{E}_{T}^{\diamond}(b)(\omega, \widetilde{\omega}):=\exp ^{\diamond} & \left(\sum_{j=1}^{d} \int_{0}^{T}\left(W_{s}^{j}(\omega)+b^{j}\left(s, \widetilde{B}_{s}(\widetilde{\omega})\right)\right) d \widetilde{B}_{s}^{j}(\widetilde{\omega})\right. \\
& \left.-\frac{1}{2} \int_{0}^{T}\left(W_{s}^{j}(\omega)+b^{j}\left(s, \widetilde{B}_{s}(\widetilde{\omega})\right)\right)^{\diamond 2} d s\right) .
\end{aligned}
$$


Here $(\widetilde{\Omega}, \widetilde{\mathcal{F}}, \widetilde{\mu}),\left(\widetilde{B}_{t}\right)_{t \geq 0}$ is a copy of the quadruple $(\Omega, \mathcal{F}, \mu),\left(B_{t}\right)_{t \geq 0}$ in (2.26). Further $E_{\widetilde{\mu}}$ denotes a Pettis integral of random elements $\Phi: \widetilde{\Omega} \longrightarrow(\mathcal{S})^{*}$ with respect to the measure $\widetilde{\mu}$. The Wick product $\diamond$ in the Wick exponential of (2.28) is taken with respect to $\mu$ and $W_{t}^{j}$ is the white noise of $B_{t}^{j}$ in the Hida space $(\mathcal{S})^{*}$ (see (2.13)). The stochastic integrals $\int_{0}^{T} \phi(t, \widetilde{\omega}) d \widetilde{B}_{s}^{j}(\widetilde{\omega})$ in (2.28) are defined for predictable integrands $\phi$ with values in the conuclear space $(\mathcal{S})^{*}$. See [19] for definitions. The other integral type in (2.28) is to be understood in the sense of Pettis.

Remark 2.2 Let $0=t_{1}^{n}<t_{2}^{n}<\ldots<t_{m_{n}}^{n}=T$ be a sequence of partitions of the interval $[0, T]$ with $\max _{i=1}^{m_{n}-1}\left|t_{i+1}^{n}-t_{i}^{n}\right| \longrightarrow 0$. Then the stochastic integral of the white noise $W^{j}$ can be approximated as follows:

$$
\int_{0}^{T} W_{s}^{j}(\omega) d \widetilde{B}_{s}^{j}(\widetilde{\omega})=\lim _{n \longrightarrow \infty} \sum_{i=1}^{m_{n}}\left(\widetilde{B}_{t_{i+1}^{n}}^{j}(\widetilde{\omega})-\widetilde{B}_{t_{i}^{n}}^{j}(\widetilde{\omega})\right) W_{t_{i}^{n}}^{j}(\omega)
$$

in $L^{2}\left(\lambda \times \widetilde{\mu} ;(\mathcal{S})^{*}\right)$. For more information about stochastic integration on conuclear spaces the reader may consult [19].

In the sequel we shall use the notation $Y_{t}^{i, b}$ for the expectation on the right hand side of (2.27) for $\varphi(t, x)=x$, that is

$$
Y_{t}^{i, b}:=E_{\widetilde{\mu}}\left[\widetilde{B}_{t}^{(i)} \mathcal{E}_{T}^{\diamond}(b)\right]
$$

for $i=1, \ldots, d$. We set

$$
Y_{t}^{b}=\left(Y_{t}^{1, b}, \ldots, Y_{t}^{d, b}\right) .
$$

The form of Formula (2.27) in Proposition 2.1 actually suggests that the expectation on the right hand side or $Y_{t}^{b}$ in (2.29) may also represent solutions of (2.25) for merely measurable drift coefficients $b$. The latter naturally leads to the following question: Can one specify conditions on $b$ under which one succeeds to directly verify the generalized process $Y_{t}^{b}$ to be a (strong) solution of (2.25)? This question was successfully treated for the onedimensional case using a comparison argument in [29] and for the multidimensional case under a rather strong symmetry condition on the drift $b$ using Malliavin calculus in [31]. In this paper we considerably improve the results given in [31] by removing the symmetry condition on $b$. Our main result in this paper is the following theorem:

Theorem 2.3 Suppose that the drift coefficient $b:[0,1] \times \mathbb{R}^{d} \rightarrow \mathbb{R}^{d}$ in $(2.25)$ is a bounded Borel-measurable function. Then there exists a unique global strong solution $X$ to Equation (2.25) such that $X_{t}$ is Malliavin differentiable for all $0 \leq t \leq 1$.

Remark 2.4 In the one-dimensional case the existence and uniqueness of strong solutions to (2.25) for bounded and measurable drift coefficients was first obtained by Zvonkin in his celebrated paper [52]. The extension to the multi-dimensional case was given by [49]. We point out that our solution technique grants the important additional insight that such solutions are Malliavin differentiable. We remark that Theorem 2.3 is a generalization of [30, Theorem 5] from the one-dimensional to the multi-dimensional case. Let us also mention that we considerably improve the technique initiated in [31] (see also [29] and [38]) by removing a certain symmetry condition on the drift coefficients in (2.25) (see [30, Definition 3]), which 
severely limits the class of SDE's to be analyzed. The removal of the latter condition, however, may actually pave the way for the construction of strong solutions of discontinuous infinite dimensional stochastic equations of the type (2.62) or SPDE's. See [28]. We point out that the methods of the authors mentioned in the introduction fail in this case.

To prove Theorem 2.3 we follow a procedure consisting of two steps (compare [31]). In the first step, we show for a sequence of uniformly bounded, smooth coefficients $b_{n}:[0,1] \times \mathbb{R}^{d} \rightarrow$ $\mathbb{R}^{d}, n \geq 1$, with compact support that for each $0 \leq t \leq 1$ the sequence of corresponding strong solutions $X_{n, t}=Y_{t}^{b_{n}}, n \geq 1$, is relatively compact in $L^{2}\left(\mu ; \mathbb{R}^{d}\right)$ (Corollary 2.6). The main tool to prove compactness is the bound in Lemma 2.5 in connection with a compactness criteria in terms of Malliavin derivatives obtained in [6] (see Appendix 2.5). This step is one of the main contribution of this paper.

Given a merely measurable and bounded drift coefficient $b$, we then show in the second step that $Y_{t}^{b}, 0 \leq t \leq 1$ is a generalized process in the Hida distribution space, and we apply the $S$-transform 2.14 to prove that for a given sequence of a.e. approximating, uniformly bounded, smooth coefficients $b_{n}$ with compact support a subsequence of the corresponding strong solutions $X_{n_{j}, t}=Y_{t}^{b_{n_{j}}}$ fulfills

$$
Y_{t}^{b_{n_{j}}} \rightarrow Y_{t}^{b}
$$

in $L^{2}\left(\mu ; \mathbb{R}^{d}\right)$ for $0 \leq t \leq 1$ (Lemma 2.14). Using a certain transformation property for $Y_{t}^{b}$ (Lemma 2.16) we directly verify $Y_{t}^{b}$ as a solution to (2.25) which in addition is Malliavin differentiable.

We now turn to the first step of our procedure. The successful completion of the first step relies on the following essential lemma:

Lemma 2.5 Let $b:[0,1] \times \mathbb{R}^{d} \rightarrow \mathbb{R}^{d}$ be a smooth function with compact support. Then the corresponding strong solution $X$ in (2.25) fulfills

$$
E\left[\left\|D_{t} X_{s}-D_{t^{\prime}} X_{s}\right\|^{2}\right] \leq C_{d}\left(\|b\|_{\infty}\right)\left|t-t^{\prime}\right|^{\alpha}
$$

for $0 \leq t^{\prime} \leq t \leq 1, \alpha=\alpha(s)>0$ and

$$
\sup _{0 \leq t \leq 1} E\left[\left\|D_{t} X_{s}\right\|^{2}\right] \leq C_{d}\left(\|b\|_{\infty}\right)
$$

where $C_{d}:[0, \infty) \rightarrow[0, \infty)$ is an increasing, continuous function, $\|\cdot\|$ a matrix-norm on $\mathbb{R}^{d \times d}$ and $\|\cdot\|_{\infty}$ the supremum norm.

From Lemma 2.5 together with Corollary 2.30 we immediately obtain the main result of step one of our procedure:

Corollary 2.6 Let $b_{n}:[0,1] \times \mathbb{R}^{d} \rightarrow \mathbb{R}^{d}, n \geq 1$, be a sequence of uniformly bounded, smooth coefficients with compact support. Then for each $0 \leq t \leq 1$ the sequence of corresponding strong solutions $X_{n, t}=Y_{t}^{b_{n}}, n \geq 1$, is relatively compact in $L^{2}\left(\mu ; \mathbb{R}^{d}\right)$.

In order to prove Lemma 2.5 we need the following estimate, which can be considered a generalization of a bound given in [7, Proposition 2.2]: 
Proposition 2.7 Let $B$ be a d-dimensional Brownian Motion starting from the origin and $b_{1}, \ldots, b_{n}$ be compactly supported continuously differentiable functions $b_{i}:[0,1] \times \mathbb{R}^{d} \rightarrow \mathbb{R}$ for $i=1,2, \ldots n$. Let $\alpha_{i} \in\{0,1\}^{d}$ be a multiindex such that $\left|\alpha_{i}\right|=1$ for $i=1,2, \ldots, n$. Then there exists a universal constant $C$ (independent of $\left\{b_{i}\right\}_{i}, n$, and $\left\{\alpha_{i}\right\}_{i}$ ) such that

$$
\left|E\left[\int_{t_{0}<t_{1}<\cdots<t_{n}<t}\left(\prod_{i=1}^{n} D^{\alpha_{i}} b_{i}\left(t_{i}, B\left(t_{i}\right)\right)\right) d t_{1} \ldots d t_{n}\right]\right| \leq \frac{C^{n} \prod_{i=1}^{n}\left\|b_{i}\right\|_{\infty}\left(t-t_{0}\right)^{n / 2}}{\Gamma\left(\frac{n}{2}+1\right)}
$$

where $\Gamma$ is the Gamma-function. Here $D^{\alpha_{i}}$ denotes the partial derivative with respect to the $j^{\prime}$ th space variable, where $j$ is the position of the 1 in $\alpha_{i}$.

Proof. Without loss of generality, assume that $\left\|b_{i}\right\|_{\infty} \leq 1$ for $i=1,2 \ldots, n$. Denote by $z=\left(z^{(1)}, \ldots z^{(d)}\right)$ a generic element of $\mathbb{R}^{d}$ and by $\|\cdot\|$ the usual Euclidian norm. With $P(t, z)=(2 \pi t)^{-d / 2} e^{-\|z\|^{2} / 2 t}$, write the left hand side in (2.30) as

$$
\left|\int_{t_{0}<t_{1}<\cdots<t_{n}<t} \int_{\mathbb{R}^{d n}} \prod_{i=1}^{n} D^{\alpha_{i}} b_{i}\left(t_{i}, z_{i}\right) P\left(t_{i}-t_{i-1}, z_{i}-z_{i-1}\right) d z_{1} \ldots d z_{n} d t_{1} \ldots d t_{n}\right| .
$$

Introduce the notation

$$
J_{n}^{\alpha}\left(t_{0}, t, z_{0}\right)=\int_{t_{0}<t_{1}<\cdots<t_{n}<t} \int_{\mathbb{R}^{d n}} \prod_{i=1}^{n} D^{\alpha_{i}} b_{i}\left(t_{i}, z_{i}\right) P\left(t_{i}-t_{i-1}, z_{i}-z_{i-1}\right) d z_{1} \ldots d z_{n} d t_{1} \ldots d t_{n}
$$

where $\alpha=\left(\alpha_{1}, \ldots \alpha_{n}\right) \in\{0,1\}^{n d}$. We shall show that $\left|J_{n}^{\alpha}\left(t_{0}, t, 0\right)\right| \leq C^{n}\left(t-t_{0}\right)^{n / 2} / \Gamma(n / 2+1)$, thus proving the proposition.

To do this, we will use integration by parts to shift the derivatives onto the Gaussian kernel. This will be done by introducing the alphabet

$$
\mathcal{A}(\alpha)=\left\{P, D^{\alpha_{1}} P, \ldots, D^{\alpha_{n}} P, D^{\alpha_{1}} D^{\alpha_{2}} P, \ldots D^{\alpha_{n-1}} D^{\alpha_{n}} P\right\}
$$

where $D^{\alpha_{i}}, D^{\alpha_{i}} D^{\alpha_{i+1}}$ denotes the derivatives in $z$ on $P(t, z)$.

Take a string $S=S_{1} \cdots S_{n}$ in $\mathcal{A}(\alpha)$ and define

$$
I_{S}^{\alpha}\left(t_{0}, t, z_{0}\right)=\int_{t_{0}<\cdots<t_{n}<t} \int_{\mathbb{R}^{d n}} \prod_{i=1}^{n} b_{i}\left(t_{i}, z_{i}\right) S_{i}\left(t_{i}-t_{i-1}, z_{i}-z_{i-1}\right) d z_{1} \ldots d z_{n} d t_{1} \ldots d t_{n} .
$$

We will only need a special type of strings, and we say that a string is allowed if, when all the $D^{\alpha_{i}} P$ 's are removed from the string, a string of the form $P \cdot D^{\alpha_{s}} D^{\alpha_{s+1}} P \cdot P \cdot D^{\alpha_{s+1}} D^{\alpha_{s+2}} P \cdots P$. $D^{\alpha_{r}} D^{\alpha_{r+1}} P$ for $s \geq 1, r \leq n-1$ remains. Also, we will require that the first derivatives $D^{\alpha_{i}} P$ are written in an increasing order with respect to $i$.

Before we proceed with the proof of Proposition 2.7 we will need some intermediate results.

Lemma 2.8 We can write

$$
J_{n}^{\alpha}\left(t_{0}, t, z_{0}\right)=\sum_{j=1}^{2^{n-1}} \epsilon_{j} I_{S^{j}}^{\alpha}\left(t_{0}, t, z_{0}\right)
$$

where each $\epsilon_{j}$ is either -1 or 1 and each $S^{j}$ is an allowed string in $\mathcal{A}(\alpha)$. 
Proof. The equation obviously holds for $n=1$. Assume the equation holds for $n \geq 1$, and let $b_{0}$ be another function satisfying the requirements of the proposition. Likewise with $\alpha_{0}$. Then

$$
\begin{aligned}
J_{n+1}^{\left(\alpha_{0}, \alpha\right)}\left(t_{0}, t, z_{0}\right)= & \int_{t_{0}}^{t} \int_{\mathbb{R}^{d}} D^{\alpha_{0}} b_{0}\left(t_{1}, z_{1}\right) P\left(t_{1}-t_{0}, z_{1}-z_{0}\right) J_{n}^{\alpha}\left(t_{1}, t, z_{1}\right) d z_{1} d t_{1} \\
= & -\int_{t_{0}}^{t} \int_{\mathbb{R}^{d}} b_{0}\left(t_{1}, z_{1}\right) D^{\alpha_{0}} P\left(t_{1}-t_{0}, z_{1}-z_{0}\right) J_{n}^{\alpha}\left(t_{1}, t, z_{1}\right) d z_{1} d t_{1} \\
& -\int_{t_{0}}^{t} \int_{\mathbb{R}^{d}} b_{0}\left(t_{1}, z_{1}\right) P\left(t_{1}-t_{0}, z_{1}-z_{0}\right) D^{\alpha_{0}} J_{n}^{\alpha}\left(t_{1}, t, z_{1}\right) d z_{1} d t_{1} .
\end{aligned}
$$

Notice that

$$
D^{\alpha_{0}} I_{S}^{\alpha}\left(t_{1}, t, z_{1}\right)=-I_{\tilde{S}}^{\left(\alpha_{0}, \alpha\right)}\left(t_{1}, t, z_{1}\right)
$$

where

$$
\tilde{S}= \begin{cases}D^{\alpha_{0}} P \cdot S_{2} \cdots S_{n} & \text { if } S=P \cdot S_{2} \cdots S_{n} \\ D^{\alpha_{0}} D^{\alpha_{1}} P \cdot S_{2} \cdots S_{n} & \text { if } S=D^{\alpha_{1}} P \cdot S_{2} \cdots S_{n}\end{cases}
$$

Here, $\tilde{S}$ is not an allowed string in $\mathcal{A}(\alpha)$. So from the induction hyptothesis $D^{\alpha_{0}} J_{n}^{\alpha}\left(t_{0}, t, z_{0}\right)=$ $\sum_{j=1}^{2^{n-1}}-\epsilon_{j} I_{\tilde{S}}^{\left(\alpha_{0}, \alpha\right)}\left(t_{0}, t, z_{0}\right)$ this gives

$$
J_{n+1}^{\left(\alpha_{0}, \alpha\right)}=\sum_{j=1}^{2^{n-1}}-\epsilon_{j} I_{D^{\alpha} 0 P \cdot S^{j}}^{\left(\alpha_{0}, \alpha\right)}+\sum_{j=1}^{2^{n-1}} \epsilon_{j} I_{P \cdot \tilde{S}^{j}} .
$$

It is easily checked that when $S^{j}$ is an allowed string in $\mathcal{A}(\alpha)$, both $D^{\alpha_{0}} P \cdot S^{j}$ and $P \cdot \tilde{S}^{j}$ are allowed strings in $\mathcal{A}\left(\alpha_{0}, \alpha\right)$.

For the rest of the proof of Proposition 2.7 we will bound $I_{S}^{\alpha}$ when $S$ is an allowed string, and the result will follow from the above representation.

Lemma 2.9 Let $\phi, h:[0,1] \times \mathbb{R}^{d} \rightarrow \mathbb{R}$ be measurable functions such that $|\phi(s, z)| \leq e^{-\|z\|^{2} / 3 s}$ and $\|h\|_{\infty} \leq 1$. Also let $\alpha, \beta \in\{0,1\}^{d}$ be multiindices such that $|\alpha|=|\beta|=1$. Then there exists a universal constant $C$ (independent of $\phi, h, \alpha$ and $\beta$ ) such that

$$
\left|\int_{1 / 2}^{1} \int_{t / 2}^{t} \int_{\mathbb{R}^{d}} \int_{\mathbb{R}^{d}} \phi(s, z) h(t, y) D^{\alpha} D^{\beta} P(t-s, y-z) d y d z d s d t\right| \leq C .
$$

Proof. Let $l, m \in \mathbb{Z}^{d}$ and denote $[l, l+1):=\left[l^{(1)}, l^{(1)}+1\right) \times \cdots \times\left[l^{(d)}, l^{(d)}+1\right)$ and similarly for $[m, m+1)$. Define $\phi_{l}(s, z)=\phi(s, z) 1_{[l, l+1)}(z)$ and $h_{m}(t, y)=h(t, y) 1_{[m, m+1)}(y)$.

Denote the above integral by $I$, and $I_{l, m}$ the integral when $\phi, h$ is replaced by $\phi_{l}, h_{m}$. Then we can write $I=\sum_{l, m \in \mathbb{Z}^{d}} I_{l, m}$. Below we let $C$ be a generic constant that may vary from line to line.

Assume $\|l-m\|_{\infty}:=\max _{i}\left|l^{(i)}-m^{(i)}\right| \geq 2$. For $z \in[l, l+1)$ and $y \in[m, m+1)$ we have $\|z-y\| \geq\|l-m\|_{\infty}-1$. If $\alpha \neq \beta$ we have that

$$
D^{\alpha} D^{\beta} P(t-s, z-y)=\frac{\left(z^{(i)}-y^{(i)}\right)\left(z^{(j)}-y^{(j)}\right)}{(t-s)^{2}} P(t-s, y-z)
$$


for a suitable choice of $i, j$. Then we can find $C$ such that

$$
\left|D^{\alpha} D^{\beta} P(t-s, z-y)\right| \leq C e^{-\left(\|l-m\|_{\infty}-2\right)^{2} / 4} .
$$

If $\alpha=\beta$, we have

$$
\left(D^{\alpha}\right)^{2} P(t-s, y-z)=\left(\frac{\left(y^{(i)}-z^{(i)}\right)^{2}}{t-s}-1\right) \frac{P(t-s, y-z)}{t-s}
$$

and similarily we find $C$ such that

$$
\left|\left(D^{\alpha}\right)^{2} P(t-s, y-z)\right| \leq C e^{-\left(\|l-m\|_{\infty}-2\right)^{2} / 4} .
$$

In both cases we have $\left|I_{l, m}\right| \leq C e^{-\|l\|^{2} / 8} e^{-\left(\|l-m\|_{\infty}-2\right)^{2} / 4}$ and it follows that

$$
\sum_{\|l-m\|_{\infty} \geq 2}\left|I_{l, m}\right| \leq C
$$

Assume $\|l-m\|_{\infty} \leq 1$ and let $\hat{\phi}_{l}(s, u)$ and $\hat{h}_{m}(t, u)$ be the Fourier transform in the second variable, defined by

$$
\hat{h}_{m}(t, u):=(2 \pi)^{-d / 2} \int_{\mathbb{R}^{d}} h(t, x) e^{-i(u, x)} d x
$$

and similar for $\hat{\phi}_{l}(s, u)$. By the Plancherel theorem we have that

$$
\int_{\mathbb{R}^{d}} \hat{\phi}_{l}(s, u)^{2} d u=\int_{\mathbb{R}^{d}} \phi_{l}(s, z)^{2} d z \leq C e^{-\|l\|^{2} / 6}
$$

for all $s \in[0,1]$ and

$$
\int_{\mathbb{R}^{d}} \hat{h}_{m}(t, u)^{2} d u=\int_{\mathbb{R}^{d}} h_{m}(t, y)^{2} d y \leq 1 .
$$

We can write

$$
I_{l, m}=\int_{1 / 2}^{1} \int_{t / 2}^{t} \int_{\mathbb{R}^{d}} \hat{\phi}_{l}(s, u) \hat{h}_{m}(t,-u) u^{(i)} u^{(j)}(t-s) e^{-(t-s)\|u\|^{2} / 2} d u d s d t .
$$

To see this, start with the right hand side. Then we have by Fubini

$$
\begin{aligned}
& \int_{\mathbb{R}^{d}} \hat{h}_{m}(t,-u) \hat{\phi}_{l}(s, u) u^{i} u^{j}(t-s) e^{-(t-s)\|u\|^{2} / 2} d u \\
& =(2 \pi)^{-d} \int_{\mathbb{R}^{d}} \int_{\mathbb{R}^{d}} \int_{\mathbb{R}^{d}} h_{m}(t, x) e^{i(u, x)} \phi_{l}(s, y) e^{-i(u, y)} u^{i} u^{j}(t-s) e^{-(t-s)\|u\|^{2} / 2} d u d x d y= \\
& =\int_{\mathbb{R}^{d}} \int_{\mathbb{R}^{d}} h_{m}(t, x) \phi_{l}(s, y)(t-s)\left[(2 \pi)^{-d} \int_{\mathbb{R}^{d}} e^{i(u, x-y)} u^{i} u^{j} e^{-(t-s)\|u\|^{2} / 2} d u\right] d x d y .
\end{aligned}
$$


Now look at the expression in the square brackets. Substitute $v=\sqrt{t-s} u$ to get

$$
\begin{aligned}
& (2 \pi)^{-d} \int_{\mathbb{R}^{d}} e^{i(u, x-y)} u^{i} u^{j} e^{-(t-s)\|u\|^{2} / 2} d u \\
& =(2 \pi)^{-d}(t-s)^{-d / 2} \int_{\mathbb{R}^{d}} e^{i\left(\frac{v}{\sqrt{t-s}}, x-y\right)} \frac{v^{i}}{\sqrt{t-s}} \frac{v^{j}}{\sqrt{t-s}} e^{-\|v\|^{2} / 2} d v \\
& =(2 \pi)^{-d}(t-s)^{-d / 2}(t-s)^{-1} \int_{\mathbb{R}^{d}} e^{i\left(v, \frac{x-y}{\sqrt{t-s}}\right)} v^{i} v^{j} e^{-\|v\|^{2} / 2} d v .
\end{aligned}
$$

Now put $f(v)=e^{-\|v\|^{2} / 2}$ and $p(v)=v^{(i)} v^{(j)}$. From properties of the Fourier transform we know that $\widehat{p f}=D^{\alpha} D^{\beta} \hat{f}$ and $\hat{f}=f$. This gives that the above expression is equal to

$$
(2 \pi)^{-d / 2}(t-s)^{-d / 2}(t-s)^{-1} D^{\alpha} D^{\beta} f\left(\frac{x-y}{\sqrt{t-s}}\right)=(t-s)^{-1} D^{\alpha} D^{\beta} P(t-s, x-y) .
$$

This gives the equation (2.31).

Applying $a b \leq \frac{1}{2} a^{2} c+\frac{1}{2} b^{2} c^{-1}$ with $a=\hat{\phi}_{l}(s, u) u^{(i)}, b=\hat{h}_{m}(t,-u) u^{(j)}$ and $c=e^{\|l\|^{2} / 12}$ we get

$$
\begin{aligned}
\left|I_{l, m}\right| & \leq \frac{1}{2} \int_{1 / 2}^{1} \int_{t / 2}^{t} \int_{\mathbb{R}^{d}} \hat{\phi}_{l}(s, u)^{2}\left(u^{(i)}\right)^{2} e^{\|l\|^{2} / 12} e^{-(t-s)\|u\|^{2} / 2} d u d s d t \\
& +\frac{1}{2} \int_{1 / 2}^{1} \int_{t / 2}^{t} \int_{\mathbb{R}^{d}} \hat{h}_{m}(t,-u)^{2}\left(u^{(j)}\right)^{2} e^{-\|l\|^{2} / 12} e^{-(t-s)\|u\|^{2} / 2} d u d s d t \\
& \leq \frac{1}{2} \int_{1 / 2}^{1} \int_{t / 2}^{t} \int_{\mathbb{R}^{d}} \hat{\phi}_{l}(s, u)^{2}\|u\|^{2} e^{\|l\|^{2} / 12} e^{-(t-s)\|u\|^{2} / 2} d u d s d t \\
& +\frac{1}{2} \int_{1 / 2}^{1} \int_{t / 2}^{t} \int_{\mathbb{R}^{d}} \hat{h}_{m}(t,-u)^{2}\|u\|^{2} e^{-\|l\|^{2} / 12} e^{-(t-s)\|u\|^{2} / 2} d u d s d t .
\end{aligned}
$$

For the first term, integrate first with respect to $t$ in order to get

$$
\int_{1 / 2}^{1} \int_{t / 2}^{t} \int_{\mathbb{R}^{d}} \hat{\phi}_{l}(s, u)^{2}\|u\|^{2} e^{\|l\|^{2} / 12} e^{-(t-s)\|u\|^{2} / 2} d u d s d t \leq C e^{-\|l\|^{2} / 12}
$$

and for the second term, integrate with respect to $s$ first to get

$$
\int_{1 / 2}^{1} \int_{t / 2}^{t} \int_{\mathbb{R}^{d}} \hat{h}_{m}(t,-u)^{2}\|u\|^{2} e^{-\|l\|^{2} / 12} e^{-(t-s)\|u\|^{2} / 2} d u d s d t \leq C e^{-\|l\|^{2} / 12}
$$

which gives $\left|I_{l, m}\right| \leq C e^{-\|l\|^{2} / 12}$ and hence

$$
\sum_{\|l-m\|_{\infty} \leq 1}\left|I_{l, m}\right| \leq C
$$

Corollary 2.10 There exists an absolute constant $C$ such that for measurable functions $g$ and $h$ bounded by 1

$$
\left|\int_{1 / 2}^{1} \int_{t / 2}^{t} \int_{\mathbb{R}^{d}} \int_{\mathbb{R}^{d}} g(s, z) P(s, z) h(t, y) D^{\alpha} D^{\beta} P(t-s, y-z) d y d z d s d t\right| \leq C
$$


and

$$
\left|\int_{1 / 2}^{1} \int_{t / 2}^{t} \int_{\mathbb{R}^{d}} \int_{\mathbb{R}^{d}} g(s, z) D^{\gamma} P(s, z) h(t, y) D^{\alpha} D^{\beta} P(t-s, y-z) d y d z d s d t\right| \leq C .
$$

Notice that we have $\int_{\mathbb{R}^{d}} P(t, z) d z=1$ and that

$$
\begin{gathered}
\int_{\mathbb{R}^{d}}\left|D^{\alpha} P(t, z)\right| d z \leq C t^{-1 / 2}, \\
\int_{\mathbb{R}^{d}}\left|D^{\alpha} D^{\beta} P(t, z)\right| d z \leq C t^{-1} .
\end{gathered}
$$

Lemma 2.11 There is an absolute constant $C$ such that for every Borel-measurable functions $g$ and $h$ bounded by 1 , and $r \geq 0$

$$
\begin{array}{r}
\left|\int_{t_{0}}^{t} \int_{t_{0}}^{t_{1}} \int_{\mathbb{R}^{d}} \int_{\mathbb{R}^{d}} g\left(t_{2}, z\right) P\left(t_{2}-t_{0}, z\right) h\left(t_{1}, y\right) D^{\alpha} D^{\beta} P\left(t_{1}-t_{2}, y-z\right)\left(t-t_{1}\right)^{r} d y d z d t_{2} d t_{1}\right| \\
\leq C(1+r)^{-1}\left(t-t_{0}\right)^{r+1}
\end{array}
$$

and

$$
\begin{array}{r}
\left|\int_{t_{0}}^{t} \int_{t_{0}}^{t_{1}} \int_{\mathbb{R}^{d}} \int_{\mathbb{R}^{d}} g\left(t_{2}, z\right) D^{\gamma} E\left(t_{2}-t_{0}, z\right) h\left(t_{1}, y\right) D^{\alpha} D^{\beta} P\left(t_{1}-t_{2}, y-z\right)\left(t-t_{1}\right)^{r} d y d z d t_{2} d t_{1}\right| \\
\leq C(1+r)^{-1 / 2}\left(t-t_{0}\right)^{r+1 / 2} .
\end{array}
$$

Proof. We begin by proving the estimate for $t=1, t_{0}=0$. From Corollary 2.10 we have that for each $k \geq 0$

$$
\begin{array}{r}
\left|\int_{2^{-k-1}}^{2^{-k}} \int_{t / 2}^{t} \int_{\mathbb{R}^{d}} \int_{\mathbb{R}^{d}} g(s, z) P(s, z) h(t, y) D^{\alpha} D^{\beta} P(t-s, y-z)(1-t)^{r} d y d z d s d t\right| \\
\leq C\left(1-2^{-k-1}\right)^{r} 2^{-k} .
\end{array}
$$

To see this, make the substitutions $t^{\prime}=2^{k} t$ and $s^{\prime}=2^{k} s$. Use the easily verified fact that $P(a t, z)=a^{-d / 2} P\left(t, a^{-1 / 2} z\right)$ and substitute $z^{\prime}=2^{k / 2} z$ and $y^{\prime}=2^{k / 2} y$. Using $\tilde{h}(t, y):=$ $\frac{(1-t)^{r}}{\left(1-2^{-k-1}\right)^{r}} h(t, y)$ in Corollary 2.10, the result follows.

Summing this equation over $k$ gives

$$
\left|\int_{0}^{1} \int_{t / 2}^{t} \int_{\mathbb{R}^{d}} \int_{\mathbb{R}^{d}} g(s, z) P(s, z) h(t, y) D^{\alpha} D^{\beta} P(t-s, y-z)(1-t)^{r} d y d z d s d t\right| \leq C(1+r)^{-1}
$$

Moreover from the bound (2.33)

$$
\begin{array}{r}
\left|\int_{0}^{1} \int_{0}^{t / 2} \int_{\mathbb{R}^{d}} \int_{\mathbb{R}^{d}} g(s, z) P(s, z) h(t, y) D^{\alpha} D^{\beta} P(t-s, y-z)(1-t)^{r} d y d z d s d t\right| \\
\leq C \int_{0}^{1} \int_{0}^{t / 2}(t-s)^{-1}(1-t)^{r} d s d t \leq C(1+r)^{-1}
\end{array}
$$


and combining these bounds gives the first assertion for $t=1, t_{0}=0$. For general $t$ and $t_{0}$ use the change of variables $t_{1}^{\prime}=\frac{t_{1}-t_{0}}{t-t_{0}}, t_{2}=\frac{t_{2}-t_{0}}{t-t_{0}}, y^{\prime}=\left(t-t_{0}\right)^{-1 / 2} y$ and $z^{\prime}=\left(t-t_{0}\right)^{-1 / 2} z$.

The second assertion is proved similary.

We turn to the completion of the proof of Proposition 2.7 by showing that there exists a constant $M$ such that for each allowed string $S$ in the alphabet $\mathcal{A}(\alpha)$ we have

$$
I_{S}^{\alpha}\left(t_{0}, t, z_{0}\right) \leq \frac{M^{n}\left(t-t_{0}\right)^{n / 2}}{\Gamma\left(\frac{n}{2}+1\right)} .
$$

We will prove this by induction on $n$. The case $n=0$ is immediate, so assume $n>0$ and that this holds for all allowed strings of length less than $n$. There are three cases

1. $S=D^{\alpha_{1}} P \cdot S^{\prime}$ where $S^{\prime}$ is a string in $\mathcal{A}\left(\alpha^{\prime}\right)$ and $\alpha^{\prime}:=\left(\alpha_{2}, \ldots, \alpha_{n}\right)$

2. $S=P \cdot D^{\alpha_{1}} D^{\alpha_{2}} P \cdot S^{\prime}$ where $S^{\prime}$ is a string in $\mathcal{A}\left(\alpha^{\prime}\right)$ and $\alpha^{\prime}:=\left(\alpha_{3}, \ldots, \alpha_{n}\right)$

3. $S=P \cdot D^{\alpha_{1}} P \cdots D^{\alpha_{m}} P \cdot D^{\alpha_{m+1}} D^{\alpha_{m+2}} P \cdot S^{\prime}$ where $S^{\prime}$ is a string in $\mathcal{A}\left(\alpha^{\prime}\right)$ and $\alpha^{\prime}:=$ $\left(\alpha_{m+3}, \ldots, \alpha_{n}\right)$.

In each case, $S^{\prime}$ is an allowed string in the given alphabet.

1. We use the inductive hypothesis to bound $I_{S^{\prime}}^{\alpha^{\prime}}\left(t_{1}, t, z_{1}\right)$ and the bound $(2.32)$ to get

$$
\begin{aligned}
\left|I_{S}^{\alpha}\left(t_{0}, t, z_{0}\right)\right| & =\left|\int_{t_{0}}^{t} \int_{\mathbb{R}^{d}} b_{1}\left(t_{1}, z_{1}\right) D^{\alpha_{1}} P\left(t_{1}-t_{0}, z_{1}-z_{0}\right) I_{S^{\prime}}^{\alpha^{\prime}}\left(t_{1}, t, z_{1}\right) d z_{1} d t_{1}\right| \\
& \leq \frac{M^{n-1}}{\Gamma\left(\frac{n+1}{2}\right)} \int_{t_{0}}^{t}\left(t-t_{1}\right)^{(n-1) / 2} \int_{\mathbb{R}^{d}}\left|D^{\alpha_{1}} P\left(t_{1}-t_{0}, z_{1}-z_{0}\right)\right| d z_{1} d t_{1} \\
& \leq \frac{M^{n-1} C}{\Gamma\left(\frac{n+1}{2}\right)} \int_{t_{0}}^{t}\left(t-t_{1}\right)^{(n-1) / 2}\left(t_{1}-t_{0}\right)^{-1 / 2} d t_{1} \\
& =\frac{M^{n-1} C \sqrt{\pi}\left(t-t_{0}\right)^{k / 2}}{\Gamma\left(\frac{n}{2}+1\right)} .
\end{aligned}
$$

The result follows if $M$ is large enough.

2. For this case we can write

$$
\begin{aligned}
& I_{S}^{\alpha}\left(t_{0}, t, z_{0}\right)=\int_{t_{0}}^{t} \int_{t_{1}}^{t} \int_{\mathbb{R}^{d}} \int_{\mathbb{R}^{d}} b_{1}\left(t_{1}, z_{1}\right) b_{2}\left(t_{2}, z_{2}\right) \\
& \quad \times P\left(t_{1}-t_{0}, z_{1}-z_{0}\right) D^{\alpha_{1}} D^{\alpha_{2}} P\left(t_{2}-t_{1}, z_{2}-z_{1}\right) I_{S^{\prime}}^{\alpha^{\prime}}\left(t_{2}, t, z_{2}\right) d z_{1} d z_{2} d t_{2} d t_{1} .
\end{aligned}
$$

We set $h\left(t_{2}, z_{2}\right):=b_{2}\left(t_{2}, z_{2}\right) I_{S^{\prime}}^{\alpha^{\prime}}\left(t_{2}, z_{2}\right)\left(t-t_{2}\right)^{1-n / 2}$ so that by the inductive hypothesis we have

$$
\|h\|_{\infty} \leq M^{n-2} / \Gamma(n / 2) .
$$

Use this in the first part of Lemma 2.11 with $g=b_{1}$ and integrate with respect to $t_{2}$ first, to get

$$
\left|I_{S}^{\alpha}\left(t_{0}, t, z_{0}\right)\right| \leq \frac{C M^{n-2}\left(t-t_{0}\right)^{n / 2}}{n \Gamma(n / 2)},
$$

and the result follows if $M$ is large enough. 
3. We have

$$
\begin{aligned}
I_{S}^{\alpha}\left(t_{0}, t, z_{0}\right) & =\int_{t_{0}<\ldots t_{m+2}<t} \int_{\mathbb{R}^{(m+2) d}} P\left(t_{1}-t_{0}, z_{1}-z_{0}\right) \prod_{j=1}^{m+2} b_{j}\left(t_{j}, z_{j}\right) \\
& \times \prod_{j=2}^{m} D^{\alpha_{j}} P\left(t_{j}-t_{j-1}, z_{j}-z_{j-1}\right) D^{\alpha_{m+1}} D^{\alpha_{m+2}} P\left(t_{m+2}-t_{m+1}, z_{m+2}-z_{m+1}\right) \\
& \times I_{S^{\prime}}^{\alpha^{\prime}}\left(t_{m+2}, t, z_{m+2}\right) d z_{1} \ldots d z_{m+2} d t_{1} \ldots d t_{m+2} .
\end{aligned}
$$

Let $h\left(t_{m+2}, z_{m+2}\right)=b_{m+2}\left(t_{m+2}, z_{m+2}\right) I_{S^{\prime}}^{\alpha^{\prime}}\left(t_{m+2}, t, z\right)\left(t-t_{m+2}\right)^{(2+m-n) / 2}$, so that from the inductive hypothesis we have $\|h\|_{\infty} \leq M^{n-m-2} / \Gamma((n-m) / 2)$. Write

$$
\begin{aligned}
\Omega\left(t_{m}, z_{m}\right) & :=\int_{t_{m}}^{t} \int_{t_{m+1}}^{t} \int_{\mathbb{R}^{2 d}} b_{m+1}\left(t_{m+1}, z_{m+1}\right) h\left(t_{m+2}, z_{m+2}\right) \\
& \times\left(t-t_{m+2}\right)^{(n-m-2) / 2} D^{\alpha_{m}} P\left(t_{m+1}-t_{m}, z_{m+1}-z\right) \\
& \times D^{\alpha_{m+1}} D^{\alpha_{m+2}} P\left(t_{m+2}-t_{m+1}, z_{m+2}-z_{m+1}\right) d z_{m+1} d z_{m+2} d t_{m+1} d t_{m+2}
\end{aligned}
$$

so that from Lemma (3.9) we have that

$$
\left|\Omega\left(t_{m}, z_{m}\right)\right| \leq \frac{C(n-m)^{-1 / 2} M^{n-m-2}\left(t-t_{m}\right)^{(n-m-1) / 2}}{\Gamma\left(\frac{n-m}{2}\right)} .
$$

Using this in

$$
\begin{aligned}
I_{S}^{\alpha}\left(t_{0}, t, z_{0}\right)= & \int_{t_{0}<\ldots t_{m+2}<t} \int_{\mathbb{R}^{(m+2) d}} P\left(t_{1}-t_{0}, z_{1}-z_{0}\right) \prod_{j=1}^{m} b_{j}\left(t_{j}, z_{j}\right) \\
& \times \prod_{j=1}^{m-1} D^{\alpha_{j}} P\left(t_{j}-t_{j-1}, z_{j}-z_{j-1}\right) \Omega\left(t_{m}, z_{m}\right) d z_{1} \ldots d z_{m} d t_{1} \ldots d t_{m},
\end{aligned}
$$

and using the bound (2.32) several times gives

$$
\begin{aligned}
\left|I_{S}^{\alpha}\left(t_{0}, t, z_{0}\right)\right| & \leq C^{m+1}(n-m)^{-1 / 2} \frac{M^{n-m-2}}{\Gamma((n-m) / 2)} \\
& \times \int_{t_{0}<\ldots t_{m}<t}\left(t_{2}-t_{1}\right)^{-1 / 2} \ldots\left(t_{m}-t_{m-1}\right)^{-1 / 2}\left(t-t_{m}\right)^{(n-m-1) / 2} d t_{1} \ldots d t_{m} \\
& =C^{m+1}(n-m)^{-1 / 2} \frac{M^{n-m-2} \pi^{(m-1) / 2} \Gamma\left(\frac{n-m+1}{2}\right)}{\Gamma\left(\frac{n-m}{2}\right) \Gamma\left(\frac{n}{2}+1\right)}\left(t-t_{0}\right)^{n / 2},
\end{aligned}
$$

and the result follows when $M$ is large enough, thus proving the induction step.

We are now ready to complete the proof of Lemma 2.5.

\section{Proof of Lemma 2.5.}

Using the chain-rule of the Malliavin derivative $D_{t}$ (see [34]) we find that

$$
D_{t} X_{s}=\mathcal{I}_{d}+\int_{t}^{s} b^{\prime}\left(u, X_{u}\right) D_{t} X_{u} d u
$$


$\mu$-a.e. for all $1 \geq t \geq s$, where $\mathcal{I}_{d}$ is the $d \times d$ identity matrix and $b^{\prime}=\left(\frac{\partial}{\partial x_{i}} b^{(j)}(t, x)\right)_{1 \leq i, j \leq d}$ is the (bounded) space derivative of $b$.

Fix $0 \leq t^{\prime} \leq t<1$. Then, for $1 \geq s \geq t$ we have

$$
\begin{aligned}
D_{t^{\prime}} X_{s}-D_{t} X_{s} & =\int_{t^{\prime}}^{s} b^{\prime}\left(u, X_{u}\right) D_{t^{\prime}} X_{u} d u-\int_{t}^{s} b^{\prime}\left(u, X_{u}\right) D_{t} X_{u} d u \\
& =\int_{t^{\prime}}^{t} b^{\prime}\left(u, X_{u}\right) D_{t^{\prime}} X_{u} d u+\int_{t}^{s} b^{\prime}\left(u, X_{u}\right)\left(D_{t^{\prime}} X_{u}-D_{t} X_{u}\right) d u \\
& =D_{t^{\prime}} X_{t}-\mathcal{I}_{d}+\int_{t}^{s} b^{\prime}\left(u, X_{u}\right)\left(D_{t^{\prime}} X_{u}-D_{t} X_{u}\right) d u .
\end{aligned}
$$

Applying Picard iteration to the above equation we find that

$$
\begin{aligned}
& D_{t^{\prime}} X_{s}-D_{t} X_{s} \\
& =\left(\mathcal{I}_{d}+\sum_{n=1}^{\infty} \int_{t<s_{1}<\cdots<s_{n}<s} b^{\prime}\left(s_{1}, X_{s_{1}}\right): \cdots: b^{\prime}\left(s_{n}, X_{s_{n}}\right) d s_{1} \ldots d s_{n}\right)\left(D_{t^{\prime}} X_{t}-\mathcal{I}_{d}\right)
\end{aligned}
$$

in $L^{2}(\mu)$, uniformly in $s$, where : denotes (non-commutative) matrix multiplication. On the other hand we also observe that

$$
D_{t^{\prime}} X_{t}-\mathcal{I}_{d}=\sum_{n=1}^{\infty} \int_{t^{\prime}<s_{1}<\cdots<s_{n}<t} b^{\prime}\left(s_{1}, X_{s_{1}}\right): \cdots: b^{\prime}\left(s_{n}, X_{s_{n}}\right) d s_{1} \ldots d s_{n} .
$$

Denote by $\|\cdot\|$ the maximum norm on $\mathbb{R}^{d \times d}$. Then Girsanov's theorem, Hölder's inequality and the Novikov condition in connection with (2.35) and (2.36) yield

$$
\begin{aligned}
E\left[\left\|D_{t^{\prime}} X_{s}-D_{t} X_{s}\right\|^{2}\right]= & E\left[\|\left(\mathcal{I}_{d}+\sum_{n=1}^{\infty} \int_{t<s_{1}<\cdots<s_{n}<s} b^{\prime}\left(s_{1}, B_{s_{1}}\right): \cdots: b^{\prime}\left(s_{n}, B_{s_{n}}\right) d s_{1} \ldots d s_{n}\right)\right. \\
& \times\left(\sum_{n=1}^{\infty} \int_{t^{\prime}<s_{1}<\cdots<s_{n}<t} b^{\prime}\left(s_{1}, B_{s_{1}}\right): \cdots: b^{\prime}\left(s_{n}, B_{s_{n}}\right) d s_{1} \ldots d s_{n}\right) \|^{2} \\
& \left.\times \mathcal{E}\left(\sum_{j=1}^{d} \int_{0}^{1} b^{(j)}\left(u, B_{u}\right) d B_{u}^{(j)}\right)\right] \\
\leq & C_{1}\left\|\mathcal{I}_{d}+\sum_{n=1}^{\infty} \int_{t<s_{1}<\cdots<s_{n}<s} b^{\prime}\left(s_{1}, B_{s_{1}}\right): \cdots: b^{\prime}\left(s_{n}, B_{s_{n}}\right) d s_{1} \ldots d s_{n}\right\|_{L^{8}\left(\mu ; \mathbb{R}^{d \times d}\right)}^{2} \\
& \times\left\|\sum_{n=1}^{\infty} \int_{t^{\prime}<s_{1}<\cdots<s_{n}<t} b^{\prime}\left(s_{1}, B_{s_{1}}\right): \cdots: b^{\prime}\left(s_{n}, B_{s_{n}}\right) d s_{1} \ldots d s_{n}\right\|_{L^{8}\left(\mu ; \mathbb{R}^{d \times d}\right)}^{2}
\end{aligned}
$$

where $C_{1}$ is a constant and $\mathcal{E}\left(M_{t}\right)$ denotes the Doleans-Dade exponential of a martingale $M_{t}$. 
So we obtain that

$$
\left.\begin{array}{rl}
E\left[\| D_{t^{\prime}}\right. & \left.X_{s}-D_{t} X_{s} \|^{2}\right] \\
\leq & C_{1}\left\|\mathcal{I}_{d}+\sum_{n=1}^{\infty} \int_{t<s_{1}<\cdots<s_{n}<s} b^{\prime}\left(s_{1}, B_{s_{1}}\right): \cdots: b^{\prime}\left(s_{n}, B_{s_{n}}\right) d s_{1} \ldots d s_{n}\right\|_{L^{8}\left(\mu ; \mathbb{R}^{d \times d}\right)}^{2} b^{\prime}\left(s_{1}, B_{s_{1}}\right): \cdots: b^{\prime}\left(s_{n}, B_{s_{n}}\right) d s_{1} \ldots d s_{n} \|_{L^{8}\left(\mu ; \mathbb{R}^{d \times d}\right)}^{2} \\
& \times\left\|\sum_{n=1}^{\infty} \int_{t^{\prime}<s_{1}<\cdots<s_{n}<t}\right\| \int_{t<s_{1}<\cdots<s_{n}<s} \frac{\partial}{\partial x_{l_{1}}} b^{(i)}\left(s_{1}, B_{s_{1}}\right) \frac{\partial}{\partial x_{l_{2}}} b^{\left(l_{1}\right)}\left(s_{2}, B_{s_{2}}\right) \ldots \\
\leq & C_{1}\left(1+\sum_{n=1}^{\infty} \sum_{i, j=1}^{d} \sum_{l_{1}, \ldots l_{n-1}=1}^{d} \ldots \frac{\partial}{\partial x_{j}} b^{\left(l_{n-1}\right)}\left(s_{n}, B_{s_{n}}\right) d s_{1} \ldots d s_{n} \|_{L^{8}(\mu ; \mathbb{R})}\right)^{2} \\
& \times\left(\sum_{n=1}^{\infty} \sum_{i, j=1}^{d} \sum_{l_{1}, \ldots l_{n-1}=1}^{d} \| \int_{t^{\prime}<s_{1}<\cdots<s_{n}<t} \frac{\partial}{\partial x_{l_{1}}} b^{(i)}\left(s_{1}, B_{s_{1}}\right) \frac{\partial}{\partial x_{l_{2}}} b^{\left(l_{1}\right)}\left(s_{2}, B_{s_{2}}\right) \ldots\right. \\
\ldots \frac{\partial}{\partial x_{j}} b^{\left(l_{n-1}\right)}\left(s_{n}, B_{s_{n}}\right) d s_{1} \ldots d s_{n} \| \\
L^{8}(\mu ; \mathbb{R})
\end{array}\right)^{2} .
$$

Now, look at the expression

$$
A:=\int_{t^{\prime}<s_{1}<\cdots<s_{n}<t} \frac{\partial}{\partial x_{l_{1}}} b^{(i)}\left(s_{1}, B_{s_{1}}\right) \frac{\partial}{\partial x_{l_{2}}} b^{\left(l_{1}\right)}\left(s_{2}, B_{s_{2}}\right) \ldots \frac{\partial}{\partial x_{l_{n}}} b^{\left(l_{n}\right)}\left(s_{n}, B_{s_{n}}\right) d s_{1} \ldots d s_{n} .
$$

Then, using (deterministic) integration by parts, repeatedly, one finds that $A^{2}$ can be written as a sum of at most $2^{2 n}$ summands of the form

$$
\int_{t^{\prime}<s_{1}<\cdots<s_{2 n}<t} g_{1}\left(s_{1}\right) \ldots g_{2 n}\left(s_{2 n}\right) d s_{1} \ldots d s_{2 n},
$$

where $g_{l} \in\left\{\frac{\partial}{\partial x_{j}} b^{(i)}(\cdot, B):. 1 \leq i, j \leq d\right\}, l=1,2 \ldots 2 n$. Since $A^{4}=A^{2} A^{2}$, we can argue similarly and conclude that there are at most $2^{8 n}$ such summands (of length $4 n$ ). Using this principle once more we see that $A^{8}$ can be represented as a sum of at most $2^{32 n}$ summands of the form (2.39) now with length $8 n$.

Combining this with Proposition 2.7 we get that

$$
\begin{gathered}
\| \int_{t^{\prime}<s_{1}<\cdots<s_{n}<t} \frac{\partial}{\partial x_{l_{1}}} b^{(i)}\left(s_{1}, B_{s_{1}}\right) \\
\frac{\partial}{\partial x_{l_{2}}} b^{\left(l_{1}\right)}\left(s_{2}, B_{s_{2}}\right) \ldots \frac{\partial}{\partial x_{j}} b^{\left(l_{n-1}\right)}\left(s_{n}, B_{s_{n}}\right) d s_{1} \ldots d s_{n} \|_{L^{8}(\mu ; \mathbb{R})} \\
\leq\left(\frac{2^{32 n} C^{8 n}\|b\|_{\infty}^{8 n}\left|t-t^{\prime}\right|^{4 n}}{\Gamma(4 n+1)}\right)^{1 / 8} \\
\leq \frac{2^{4 n} C^{n}\|b\|_{\infty}^{n}\left|t-t^{\prime}\right|^{n / 2}}{(4 n !)^{1 / 8}} .
\end{gathered}
$$


Then it follows from (2.37) that

$$
\begin{aligned}
E\left[\left\|D_{t} X_{s}-D_{t^{\prime}} X_{s}\right\|^{2}\right] \leq & C_{1}\left(1+\sum_{n=1}^{\infty} \frac{d^{n+2} 2^{4 n} C^{n}\|b\|_{\infty}^{n}|t-s|^{n / 2}}{(4 n !)^{1 / 8}}\right)^{2} \\
& \times\left(\sum_{n=1}^{\infty} \frac{d^{n+2} 2^{4 n} C^{n}\|b\|_{\infty}^{n}\left|t-t^{\prime}\right|^{(n-1) / 2}}{(4 n !)^{1 / 8}}\right)^{2}\left|t-t^{\prime}\right| \\
\leq & C_{d}\left(\|b\|_{\infty}\right)\left|t-t^{\prime}\right|
\end{aligned}
$$

for a function $C_{d}$ as claimed in the theorem.

Similarly, we deduce the estimate for $\sup _{0 \leq t \leq s} E\left[\left\|D_{t} X_{s}\right\|^{2}\right]$.

This concludes step one in our program and we are now coming to the second step. For a Borel-measurable, bounded coefficient $b$ we gradually show the following:

- $Y_{t}^{b}$ in $(2.29)$ is a well-defined object in the Hida distribution space $(\mathcal{S})^{*}, 0 \leq t \leq 1$, (Lemma 2.12).

- For any a.e. approximating sequence of uniformly bounded, smooth coefficients $b_{n}$ with compact support a subsequence of the corresponding strong solutions $X_{n_{j}, t}=Y_{t}^{b_{n_{j}}}$, fulfills $Y_{t}^{b_{n_{j}}} \rightarrow Y_{t}^{b}$ in $L^{2}(\mu)$ for $0 \leq t \leq 1$ (in particular $Y_{t}^{b} \in L^{2}(\mu), 0 \leq t \leq 1$ ), (Lemma 2.14).

- We apply a transformation property for $Y_{t}^{b}$ (Lemma 2.16) and identify $Y_{t}^{b}$ as a Malliavin differential strong solution to $(2.25)$.

The first lemma gives a criterion under which the process $Y_{t}^{b}$ belongs to the Hida distribution space.

Lemma 2.12 Suppose that

$$
E_{\mu}\left[\exp \left(36 \int_{0}^{1}\left\|b\left(s, B_{s}\right)\right\|^{2} d s\right)\right]<\infty
$$

where the drift $b:[0,1] \times \mathbb{R}^{d} \longrightarrow \mathbb{R}^{d}$ is measurable (in particular, (2.41) is valid for $b$ bounded). Then the coordinates of the process $Y_{t}^{b}$, defined in (2.29), that is

$$
Y_{t}^{i, b}=E_{\widetilde{\mu}}\left[\widetilde{B}_{t}^{(i)} \mathcal{E}_{T}^{\diamond}(b)\right],
$$

are elements of the Hida distribution space.

Proof. See [31]

Lemma 2.13 Let $b_{n}:[0,1] \times \mathbb{R}^{d} \longrightarrow \mathbb{R}^{d}$ be a sequence of Borel measurable functions with $b_{0}=b$ such that

$$
\sup _{n \geq 0} E\left[\exp \left(512 \int_{0}^{1}\left\|b_{n}\left(s, B_{s}\right)\right\|^{2} d s\right)\right]<\infty
$$


holds. Then

$$
\left|S\left(Y_{t}^{i, b_{n}}-Y_{t}^{i, b}\right)(\phi)\right| \leq \text { const } \cdot E\left[J_{n}\right]^{\frac{1}{2}} \cdot \exp \left(34 \int_{0}^{1}\|\phi(s)\|^{2} d s\right)
$$

for all $\phi \in\left(S_{\mathbb{C}}([0,1])\right)^{d}, i=1, \ldots, d$, where the factor $J_{n}$ is defined by

$$
\begin{aligned}
J_{n}= & \sum_{j=1}^{d}\left(2 \int_{0}^{1}\left(b_{n}^{(j)}\left(u, B_{u}\right)-b^{(j)}\left(u, B_{u}\right)\right)^{2} d u\right. \\
& \left.+\left(\int_{0}^{1}\left|\left(b_{n}^{(j)}\left(u, B_{u}\right)\right)^{2}-\left(b^{(j)}\left(u, B_{u}\right)\right)^{2}\right| d u\right)^{2}\right) .
\end{aligned}
$$

In particular, if $b_{n}$ approximates $b$ in the following sense

$$
E\left[J_{n}\right] \rightarrow 0
$$

as $n \rightarrow \infty$, it follows that

$$
Y_{t}^{b_{n}} \rightarrow Y_{t}^{b} \text { in }(\mathcal{S})^{*}
$$

as $n \rightarrow \infty$ for all $0 \leq t \leq 1, i=1, \ldots, d$.

Proof. See [31]

Lemma 2.14 Let $b_{n}:[0,1] \times \mathbb{R}^{d} \longrightarrow \mathbb{R}^{d}$ be a sequence of Borel-measurable, uniformly bounded, smooth functions with compact support which approximates a Borel-measurable, bounded coefficient $b:[0,1] \times \mathbb{R}^{d} \longrightarrow \mathbb{R}^{d}$ a.e. with respect to the Lebesgue measure. Then for any $0 \leq t \leq 1$ there exists a subsequence of the corresponding strong solutions $X_{n_{j}, t}=Y_{t}^{b_{n_{j}}}, j=1,2 \ldots$, such that

$$
Y_{t}^{b_{n_{j}}} \longrightarrow Y_{t}^{b}
$$

for $j \rightarrow \infty$ in $L^{2}(\mu)$. In particular this implies $Y_{t}^{b} \in L^{2}(\mu), 0 \leq t \leq 1$.

Proof. By Corollary 2.6 we know that there exists a subsequence $Y_{t}^{b_{n_{j}}}, j=1,2 \ldots$, converging in $L^{2}(\mu)$. Further, by boundedness obviously $E\left[J_{n_{j}}\right] \rightarrow 0$ in $(2.45)$, and thus $Y_{t}^{b_{n_{j}}} \rightarrow Y_{t}^{b}$ in $(\mathcal{S})^{*}$. But then, by uniqueness of the limit, also $Y_{t}^{b_{n_{j}}} \rightarrow Y_{t}^{b}$ in $L^{2}(\mu)$.

Remark 2.15 Note that by well known approximation results there always exists a sequence of functions $b_{n}, n \geq 1$, fulfilling the assumptions in Lemma 2.14. Then Lemma 2.14 guarantees that we are now ready to state the following "transformation property" for $Y_{t}^{b}$.

Lemma 2.16 Assume that $b:[0,1] \times \mathbb{R}^{d} \longrightarrow \mathbb{R}^{d}$ is Borel-measurable and bounded. Then

$$
\varphi^{(i)}\left(t, Y_{t}^{b}\right)=E_{\widetilde{\mu}}\left[\varphi^{(i)}\left(t, \widetilde{B}_{t}\right) \mathcal{E}_{T}^{\diamond}(b)\right]
$$

a.e. for all $0 \leq t \leq 1, i=1, \ldots, d$ and $\varphi=\left(\varphi^{(1)}, \ldots, \varphi^{(d)}\right)$ such that $\varphi\left(B_{t}\right) \in L^{2}\left(\mu ; \mathbb{R}^{d}\right)$. 
Proof. See [38, Lemma 16] or [29].

Using the above auxiliary results we can finally give the proof of Theorem 2.3 .

Proof of Theorem 2.3. We aim at employing the transformation property (2.46) of Lemma 2.16 to verify that $Y_{t}^{b}$ is a unique strong solution of the SDE (2.25). To shorten notation we set $\int_{0}^{t} \varphi(s, \omega) d B_{s}:=\sum_{j=1}^{d} \int_{0}^{t} \varphi^{(j)}(s, \omega) d B_{s}^{(j)}$ and $x=0$. Also, let $b_{n}, n=1,2, \ldots$, be a sequence of functions as required in Lemma 2.14 (see Remark 2.15).

We first remark that $Y^{b}$ has a continuous modification. The latter can be checked as follows: Since each $Y_{t}^{b_{n}}$ is a strong solution of the $\operatorname{SDE}(2.25)$ with respect to the drift $b_{n}$ we obtain from Girsanov's theorem and our assumptions that

$$
\begin{aligned}
E_{\mu}\left[\left(Y_{t}^{i, b_{n}}-Y_{u}^{i, b_{n}}\right)^{4}\right] & =E_{\widetilde{\mu}}\left[\left(\widetilde{B}_{t}^{(i)}-\widetilde{B}_{u}^{(i)}\right)^{4} \mathcal{E}\left(\int_{0}^{1} b_{n}\left(s, \widetilde{B}_{s}\right) d \widetilde{B}_{s}\right)\right] \\
& \leq \text { const } \cdot|t-u|^{2}
\end{aligned}
$$

for all $0 \leq u, t \leq 1, n \geq 1, i=1, \ldots, d$. The above constant comes from the fact that $\left\{\mathcal{E}\left(\int_{0}^{1} b_{n}\left(s, \widetilde{B}_{s}\right) d \widetilde{B}_{s}\right)\right\}_{n \geq 1}$ is bounded in $L^{2}\left(\tilde{\mu}, \mathbb{R}^{d}\right)$. To see this, we note that the DoleansDade exponential is the solution of the SDE

$$
d Z_{t}^{n}=Z_{t}^{n} b_{n}\left(t, \widetilde{B}_{t}\right) d \widetilde{B}_{t}
$$

starting at $Z_{0}^{n}=1$. So that from Itô's formula we have

$$
\begin{aligned}
E_{\tilde{\mu}}\left[\left(Z_{t}^{n}\right)^{2}\right] & =1+\int_{0}^{t} E_{\tilde{\mu}}\left[\left(Z_{s}^{n}\right)^{2}\left\|b_{n}\left(s, \widetilde{B}_{s}\right)\right\|^{2}\right] d s \\
& \leq 1+\left\|b_{n}\right\|_{\infty}^{2} \int_{0}^{t} E_{\tilde{\mu}}\left[\left(Z_{s}^{n}\right)^{2}\right] d s \\
& \leq \exp \left(t\left\|b_{n}\right\|_{\infty}^{2}\right) \\
& \leq \exp \left(t \sup _{k \geq 1}\left\|b_{k}\right\|_{\infty}^{2}\right)
\end{aligned}
$$

where the second last inequality comes from Grönwall's lemma. The last expression is bounded by assumption. By Lemma 2.14 we know that

$$
Y_{t}^{b_{n_{j}}} \longrightarrow Y_{t}^{b} \text { in } L^{2}\left(\mu ; \mathbb{R}^{d}\right)
$$

for a subsequence and hence we have almost sure convergence for a further subsequence, $0 \leq t \leq 1$. So we get that by Fatou's lemma

$$
E_{\mu}\left[\left(Y_{t}^{i, b}-Y_{u}^{i, b}\right)^{4}\right] \leq \text { const } \cdot|t-u|^{2}
$$

for all $0 \leq u, t \leq 1, i=1, \ldots, d$. Then Kolmogorov's Lemma provides a continuous modification of $Y_{t}^{b}$. 
Since $\widetilde{B}_{t}$ is a weak solution of $(2.25)$ for the drift $b(s, x)+\phi(s)$ with respect to the measure $d \mu^{*}=\mathcal{E}\left(\int_{0}^{1}\left(b\left(s, \widetilde{B}_{s}\right)+\phi(s)\right) d \widetilde{B}_{s}\right) d \mu$ we obtain that

$$
\begin{aligned}
S\left(Y_{t}^{i, b}\right)(\phi) & =E_{\widetilde{\mu}}\left[\widetilde{B}_{t}^{(i)} \mathcal{E}\left(\int_{0}^{1}\left(b\left(s, \widetilde{B}_{s}\right)+\phi(s)\right) d \widetilde{B}_{s}\right)\right] \\
& =E_{\mu^{*}}\left[\widetilde{B}_{t}^{(i)}\right] \\
& =E_{\mu^{*}}\left[\int_{0}^{1}\left(b^{(i)}\left(s, \widetilde{B}_{s}\right)+\phi^{(i)}(s)\right) d s\right] \\
& =\int_{0}^{t} E_{\widetilde{\mu}}\left[b^{(i)}\left(s, \widetilde{B}_{s}\right) \mathcal{E}\left(\int_{0}^{1}\left(b\left(u, \widetilde{B}_{u}\right)+\phi(u)\right) d \widetilde{B}_{u}\right)\right] d s+S\left(B_{t}^{(i)}\right)(\phi) .
\end{aligned}
$$

Hence the transformation property (2.46) applied to $b$ gives

$$
S\left(Y_{t}^{i, b}\right)(\phi)=S\left(\int_{0}^{t} b^{(i)}\left(u, Y_{u}^{i, b}\right) d u\right)(\phi)+S\left(B_{t}^{(i)}\right)(\phi) .
$$

Then the injectivity of $S$ implies that

$$
Y_{t}^{b}=\int_{0}^{t} b\left(s, Y_{s}^{b}\right) d s+B_{t} .
$$

The Malliavin differentiability of $Y_{t}^{b}$ follows from the fact that

$$
\sup _{n \geq 1}\left\|Y_{t}^{i, b_{n}}\right\|_{1,2} \leq M<\infty
$$

for all $i=1, \ldots, d$ and $0 \leq t \leq 1$. See e.g. [34].

On the other hand our conditions allow the application of Girsanov's theorem to any other strong solution. Then the proof of Proposition 2.1 (see e.g. [38, Proposition 1]) shows that any other solution necessarily takes the form $Y_{t}^{b}$.

Finally, we give an extension of Theorem 2.3 to a class of non-degenerate $d$-dimensional Itô-diffusions.

Theorem 2.17 Consider the time-homogeneous $\mathbb{R}^{d}$-valued SDE

$$
d X_{t}=b\left(X_{t}\right) d t+\sigma\left(X_{t}\right) d B_{t}, \quad X_{0}=x \in \mathbb{R}^{d}, \quad 0 \leq t \leq T,
$$

where the coefficients $b: \mathbb{R}^{d} \longrightarrow \mathbb{R}^{d}$ and $\sigma: \mathbb{R}^{d} \longrightarrow \mathbb{R}^{d} \times \mathbb{R}^{d}$ are Borel measurable. Require that there exists a bijection $\Lambda: \mathbb{R}^{d} \longrightarrow \mathbb{R}^{d}$, which is twice continuously differentiable. Let $\Lambda_{x}: \mathbb{R}^{d} \longrightarrow L\left(\mathbb{R}^{d}, \mathbb{R}^{d}\right)$ and $\Lambda_{x x}: \mathbb{R}^{d} \longrightarrow L\left(\mathbb{R}^{d} \times \mathbb{R}^{d}, \mathbb{R}^{d}\right)$ be the corresponding derivatives of $\Lambda$ and assume that

$$
\Lambda_{x}(y) \sigma(y)=i d_{\mathbb{R}^{d}} \text { for } y \text { a.e. }
$$

as well as

$$
\Lambda^{-1} \text { is Lipschitz continuous. }
$$


Suppose that the function $b_{*}: \mathbb{R}^{d} \longrightarrow \mathbb{R}^{d}$ given by

$$
\begin{aligned}
b_{*}(x): & =\Lambda_{x}\left(\Lambda^{-1}(x)\right)\left[b\left(\Lambda^{-1}(x)\right)\right] \\
& +\frac{1}{2} \Lambda_{x x}\left(\Lambda^{-1}(x)\right)\left[\sum_{i=1}^{d} \sigma\left(\Lambda^{-1}(x)\right)\left[e_{i}\right], \sum_{i=1}^{d} \sigma\left(\Lambda^{-1}(x)\right)\left[e_{i}\right]\right]
\end{aligned}
$$

satisfies the conditions of Theorem 2.3, where $e_{i}, i=1, \ldots, d$, is a basis of $\mathbb{R}^{d}$. Then there exists a Malliavin differentiable solution $X_{t}$ to (2.48).

Proof. The proof can be directly obtained from Itô's Lemma. See [31].

\subsection{Applications}

In this section we consider various applications of the techniques and results of the previous sections. In Subsection 2.4.1 we show that our techniques can be used to analyse Sobolev differentiability of strong solutions $X_{t}$ of the SDE (2.25) in the initial condition $x$. In Subsection 2.4.2 our results on Malliavin and Sobolev differentiability of strong solutions of SDE's are employed to derive a stochastic representation of spatial derivatives of solutions to the Kolmogorov equation (Bismut-Elworthy-Li formula, [10]) with irregular drift coefficients, which does not involve derivatives of the initial condition (2.51). The latter result might be also relevant from a numerical point of view. In Subsection 2.4.3 we account for the great flexibility of our approach to be generalized to the study of solutions of various other stochastic equations besides SDE's in finite dimension. In particular we present the applicability of our techniques and results to the problem of well-posedness of stochastic transport equations with singular coefficients.

\subsubsection{Sobolev differentiability of strong solutions of SDE's}

In the sequel we consider the solution process of the SDE (2.25) as a function of the initial value, $x \in \mathbb{R}^{d}$, denoted by $X_{t}^{x}$. We remark that the following analysis can be extended to the SDE (2.48) with non-trivial diffusion coefficient under the assumptions of Theorem 2.17. It is well known (see [22]) that when $b$ is continuously differentiable with bounded derivative, the process $X_{s}^{x}$ is $\mu$-a.e. differentiable in $x$ and its derivative satisfies the following linear ODE

$$
\frac{\partial}{\partial x} X_{s}^{x}=\mathcal{I}_{d}+\int_{0}^{s} b^{\prime}\left(u, X_{u}^{x}\right) \frac{\partial}{\partial x} X_{u}^{x} d u .
$$

Note that this equation is the same as (2.34) when $t=0$. Using this observation in connection with the same technique as in the proof of Lemma 2.5, by replacing the Malliavin derivative of $X_{s}^{x}$ by $\frac{\partial}{\partial x} X_{s}^{x}$, we get the following Lemma.

Lemma 2.18 Let $b:[0,1] \times \mathbb{R}^{d} \rightarrow \mathbb{R}^{d}$ be a smooth function with compact support. Then the corresponding strong solution $X$ in (2.25) fulfills

$$
\sup _{0 \leq s \leq 1, x \in \mathbb{R}^{d}} E\left[\left\|\frac{\partial}{\partial x} X_{s}^{x}\right\|^{2}\right] \leq C_{d}\left(\|b\|_{\infty}\right),
$$

where $C_{d}:[0, \infty) \rightarrow[0, \infty)$ is an increasing, continuous function, $\|\cdot\|$ a matrix-norm on $\mathbb{R}^{d \times d}$ and $\|\cdot\|_{\infty}$ the supremum norm. 
Applying this Lemma we obtain the following result on the (local) Sobolev differentiability of $\left(x \mapsto X_{t}^{x}\right)$ in the case of irregular $b$, which we will use in our applications in the next subsections.

Proposition 2.19 Let $b:[0,1] \times \mathbb{R}^{d} \rightarrow \mathbb{R}^{d}$ be bounded and measurable. Let $U$ be an open, bounded subset of $\mathbb{R}^{d}$. For each $t \in[0,1]$ we have

$$
\left(x \mapsto X_{t}^{x}\right) \in L^{2}\left(\Omega, W^{1,2}(U)\right),
$$

where $W^{1,2}(U)$ is the space of square integrable Sobolev-differentiable functions.

The proof of Proposition 2.19 follows the same pattern as presented in Section 3. In the following, we retain the notation from the previous section.

\section{Proof.}

We start by observing that for any $\varphi \in C_{0}^{\infty}\left(U ; \mathbb{R}^{d}\right)$ and $t \in[0,1]$ the sequence of random variables

$$
\left\langle X_{t}^{n}, \varphi\right\rangle:=\int_{U}\left\langle X_{t}^{n, x}, \varphi(x)\right\rangle_{\mathbb{R}^{d}} d x
$$

is relatively compact in $L^{2}(\Omega)$. To see this we use the compactness criterion of Corollary 4.22. Note that since the Malliavin derivative is a closed linear operator we have

$$
\begin{gathered}
E\left[\left|D_{s}^{j}\left\langle X_{t}^{n}, \varphi\right\rangle\right|^{2}\right]=\sum_{i=1}^{d}\left(\int_{U} E\left[D_{s}^{j} X_{t}^{i, n, x}\right] \varphi_{i}(x) d x\right)^{2} \\
\leq\|\varphi\|_{L^{2}\left(\mathbb{R}^{d} ; \mathbb{R}^{d}\right)}^{2} \lambda\{\operatorname{supp}(\varphi)\} \sup _{x \in \operatorname{supp}(U), 0 \leq s \leq t} E\left[\left\|D_{s} X_{t}^{n, x}\right\|^{2}\right],
\end{gathered}
$$

where $D^{j}$ is the Malliavin derivative in the direction of $B^{j}$ and where $\lambda$ denotes Lebesque measure and $\operatorname{supp}(\varphi)$ is the support of $\varphi$. Summing over $j=1, \ldots d$ in connection with Lemma 2.5 gives

$$
E\left[\left\|D_{s}\left\langle X_{t}^{n}, \varphi\right\rangle\right\|^{2}\right] \leq d\|\varphi\|_{L^{2}\left(\mathbb{R}^{d} ; \mathbb{R}^{d}\right)}^{2} \lambda\{\operatorname{supp}(\varphi)\} C_{d}\left(\left\|b_{n}\right\|_{\infty}\right) .
$$

Similarly we get

$$
E\left[\left\|D_{s}\left\langle X_{t}^{n}, \varphi\right\rangle-D_{s^{\prime}}\left\langle X_{t}^{n}, \varphi\right\rangle\right\|^{2}\right] \leq d\|\varphi\|_{L^{2}\left(\mathbb{R}^{d} ; \mathbb{R}^{d}\right)}^{2} \lambda\{\operatorname{supp}(\varphi)\} C_{d}\left(\left\|b_{n}\right\|_{\infty}\right)\left|s^{\prime}-s\right|,
$$

which shows that $\left\langle X_{t}^{n}, \varphi\right\rangle$ is relatively compact. Denote by $Y_{t}(\varphi)$ its limit after taking an (if necessary) subsequence.

Taking the $S$-transform of $\left\langle X_{t}^{n}, \varphi\right\rangle$ and $\left\langle X_{t}, \varphi\right\rangle$ we see that for any $\phi \in\left(\mathcal{S}_{\mathbb{C}}([0,1])\right)^{d}$

$$
\begin{gathered}
\left|S\left(\left\langle X_{t}^{n}, \varphi\right\rangle\right)(\phi)-S\left(\left\langle X_{t}, \varphi\right\rangle\right)(\phi)\right|^{2}=\left|\left\langle S\left(X_{t}^{n}-X_{t}\right)(\phi), \varphi\right\rangle\right|^{2} \\
\leq\|\varphi\|_{L^{2}\left(\mathbb{R}^{d} ; \mathbb{R}^{d}\right)}^{2} \int_{U}\left|S\left(X_{t}^{n, x}-X_{t}^{x}\right)(\phi)\right|^{2} d x \\
\leq\|\varphi\|_{L^{2}\left(\mathbb{R}^{d} ; \mathbb{R}^{d}\right)}^{2} \int_{U} C E\left[J_{n}(x)\right] \exp \left(68 \int_{0}^{1}\|\phi(s)\|^{2} d s\right) d x,
\end{gathered}
$$

where $C$ is a constant and

$$
J_{n}(x):=\sum_{j=1}^{d}\left(2 \int_{0}^{1}\left(b_{n}^{(j)}\left(u, x+B_{u}\right)-b^{(j)}\left(u, x+B_{u}\right)\right)^{2} d u\right.
$$




$$
\left.+\left(\int_{0}^{1}\left|\left(b_{n}^{(j)}\left(u, x+B_{u}\right)\right)^{2}-\left(b^{(j)}\left(u, x+B_{u}\right)\right)^{2}\right| d u\right)^{2}\right) .
$$

See Lemma 2.13. Since $\left\{b_{n}\right\}$ is uniformly bounded, using dominated convergence, we get that

$$
\left\langle X_{t}^{n}, \varphi\right\rangle \rightarrow\left\langle X_{t}, \varphi\right\rangle
$$

in $(\mathcal{S})^{*}$, and thus in particular weakly in $L^{2}(\Omega, \mu)$. By uniqueness of the limits we can conclude that

$$
Y(\varphi)=\left\langle X_{t}, \varphi\right\rangle \mu \text {-a.s. }
$$

thus proving the assertion.

Note that there exists a subsequence $n(k)$ such that $\left\langle X_{t}^{n(k)}, \varphi\right\rangle$ converges for every $\varphi$, that is, $n(k)$ is independent of $\varphi$. To see this, let $x=0$ and choose $n(k)$ such that

$$
X_{t}^{n(k), 0} \rightarrow X_{t}^{0}
$$

in $L^{2}(\Omega)$. If there exists $\varphi \in C_{0}^{\infty}\left(\mathbb{R}^{d} ; \mathbb{R}^{d}\right)$ and $\epsilon>0$ such that $\left\|\left\langle X_{t}^{n(k)}, \varphi\right\rangle-\left\langle X_{t}, \varphi\right\rangle\right\| \geq \epsilon$ we may by the above extract a further subsequence $\left\langle X_{t}^{n(k(j))}, \varphi\right\rangle$ converging to $\left\langle X_{t}, \varphi\right\rangle$, which gives a contradiction. From now we denote this subsequence by $n$ for simplicity.

We now proceed to prove that $\left(x \mapsto X_{t}^{x}\right) \in L^{2}\left(\Omega ; W^{1,2}\left(U ; \mathbb{R}^{d}\right)\right)$ : Because of Lemma 2.18 we get that $\left(x \mapsto X_{t}^{n, x}\right)$ is bounded in $L^{2}\left(\Omega ; W^{1,2}\left(U ; \mathbb{R}^{d}\right)\right)$, thus relatively compact in the weak topology. Then there exists a subsequence $n(k)$ such that $X_{t}^{n(k), \cdot}$ converges weakly to an $Y \in L^{2}\left(\Omega ; W^{1,2}\left(U ; \mathbb{R}^{d}\right)\right)$. Then for all $A \in \mathcal{F}$ and $\varphi \in C_{0}^{\infty}$ we have

$$
\begin{gathered}
E\left[1_{A}\left\langle X_{t}, \varphi^{\prime}\right\rangle\right]=\lim _{k \rightarrow \infty} E\left[1_{A}\left\langle X_{t}^{n(k)}, \varphi^{\prime}\right\rangle\right] \\
=\lim _{k \rightarrow \infty}-E\left[1_{A}\left\langle\frac{\partial}{\partial x} X_{t}^{n(k)}, \varphi\right\rangle\right]=-E\left[1_{A}\langle Y, \varphi\rangle\right] .
\end{gathered}
$$

Hence we have

$$
\left\langle X_{t}, \varphi^{\prime}\right\rangle=-\langle Y, \varphi\rangle \mu \text {-a.s. }
$$

Finally, we need to show that there exists a measurable set $\Omega_{0} \subset \Omega$ with full measure such that $X_{t}$ has a weak derivative on this subset. To this end choose a sequence $\left\{\varphi_{n}\right\}$ in $C^{\infty}\left(\mathbb{R}^{d} ; \mathbb{R}^{d}\right)$ dense in $W_{0}^{1,2}\left(U ; \mathbb{R}^{d}\right)$. Choose a measurable subset $\Omega_{n}$ of $\Omega$ with full measure such that $(2.50)$ holds on $\Omega_{n}$ with $\varphi$ replaced by $\varphi_{n}$. Then $\Omega_{0}:=\cap_{n \geq 1} \Omega_{n}$ satisfies the desired property.

Remark 2.20 By a similar argument as in the above proof, one can show that there exists a subsequence $n(k)$ such that $X_{t}^{n(k), x} \rightarrow X_{t}^{x}$ in $L^{2}\left(\Omega ; \mathbb{R}^{d}\right)$ for all $t$ and $x$, i.e. the choice of subsequence is independent of $t$ and $x$. From now on, we shall always use this subsequence, for simplicity denoted by $n$.

Remark 2.21 We mention that Proposition 2.19 was first studied in [32]. There the authors could even show that the solution to the SDE (2.25) gives rise to a stochastic flow of Sobolev diffeomorphisms

$$
\mathbb{R}^{d} \ni x \mapsto \phi_{s, t}(x) \in \mathbb{R}^{d}, s, t \in \mathbb{R},
$$

where $\phi_{s, t}(x)$ denotes the solution at time $t$ of the SDE (2.25) that starts in $x$ at time s. 


\subsubsection{Stochastic representation of spatial derivatives of solutions to Kolmogorov equations}

We will now study the Kolmogorov equation

$$
\frac{\partial}{\partial t} v(t, x)=\sum_{j=1}^{d} b_{j}(t, x) \frac{\partial}{\partial x_{j}} v(t, x)+\frac{1}{2} \sum_{i=1}^{d} \frac{\partial^{2}}{\partial x_{i}^{2}} v(t, x)
$$

with initial condition $v(0, x)=\Phi(x)$, where $b:[0,1] \times \mathbb{R}^{d} \rightarrow \mathbb{R}^{d}$ is bounded and measurable.

It can be shown, see [50], that when $\Phi$ is continuous and bounded there exists a solution to $(2.51)$ given by

$$
v(t, x)=E\left[\Phi\left(X_{t}^{x}\right)\right],
$$

where $v$ is a solution to the Kolmogorov Equation (2.51) which is unique among all bounded solutions in the space $\cap_{p>1} W_{l o c}^{(1,2), p}\left((0,1] \times \mathbb{R}^{d}\right) \cap C\left([0,1] \times \mathbb{R}^{d}\right)$. Here $W_{l o c}^{(1,2), p}\left((0,1] \times \mathbb{R}^{d}\right)$ denotes the space of functions that are once weakly differentiable on $(0,1]$ and twice weakly differentiable on $\mathbb{R}^{d}$, and these derivatives are locally integrable to the p-th power.

In the following we are interested in the spatial derivative $\frac{\partial}{\partial x} v$, and our aim is to use the Mallavin derivative and the spatial derivative $\frac{\partial}{\partial x} X_{t}^{x}$ to find an explicit formula for $\frac{\partial}{\partial x} v$ which does not depend on the derivative of $\Phi$.

Remark 2.22 i) The spatial derivative $\frac{\partial}{\partial x} v(t, x)$ has various important interpretations. For example, if $v(t, x)$ describes the concentration of a certain type of molecules at time $t$ and position $x$ in a medium, where $b(t, x)$ is the macroscopic velocity of motion of the medium at $t, x$, then $\frac{\partial}{\partial x} v(t, x)$ can be regarded as a sensitivity measure of the concentration of molecules with respect to its location.

ii) We remark that the representation (2.53) we derive below might be useful from a numerical point of view, since no derivative of the initial condition $\Phi$ is involved. It is e.g. conceivable to approximate $\frac{\partial}{\partial x} v(t, x)$ by using Monte-Carlo simulation. The latter is at least possible in the one-dimensional case, where $\frac{\partial}{\partial x} X_{t}^{x}$ can be written in terms of a backward stochastic integral or a local time integral without using the derivative of $b$. Compare [31].

Theorem 2.23 (Bismut-Elworthy-Li formula) Assume $\Phi \in C_{b}\left(\mathbb{R}^{d}\right)$ and let $U$ be an open, bounded subset of $\mathbb{R}^{d}$. Then the derivative of the solution to (2.51) takes the following (matrix notational) form

$$
\frac{\partial}{\partial x} v(t, x)=E\left[\Phi\left(X_{t}^{x}\right) t^{-1} \int_{0}^{t} \frac{\partial}{\partial x} X_{s}^{x} d B_{s}\right]
$$

for almost all $x \in U$ and all $t \in(0,1]$.

\section{Proof.}

Assume that $\Phi \in C_{b}^{2}\left(\mathbb{R}^{d}\right)$ (the general case of $\Phi \in C_{b}\left(\mathbb{R}^{d}\right)$ can be proved by approximation of $\Phi$ in relation (2.55)) and let $b_{n}$ and $X_{t}^{n, x}$ be as in the previous section. If we replace $b$ by $b_{n}$ in (2.51) we have the unique solution given by

$$
v_{n}(t, x)=E\left[\Phi\left(X_{t}^{n, x}\right)\right] .
$$

By using Remark 4.11 we see that $v_{n}(t, x) \rightarrow v(t, x)$ for each $t$ and $x$. 
By [34, Page 109] we know that

$$
D_{s} X_{t}^{n, x} \frac{\partial}{\partial x} X_{s}^{n, x}=\frac{\partial}{\partial x} X_{t}^{n, x},
$$

where the above product is the usual matrix product. Then we have

$$
\frac{\partial}{\partial x} X_{t}^{n, x}=t^{-1} \int_{0}^{t} D_{s} X_{t}^{n, x} \frac{\partial}{\partial x} X_{s}^{n, x} d s .
$$

Interchanging integration and differentiation we get

$$
\begin{aligned}
\frac{\partial}{\partial x} v_{n}(t, x) & =E\left[\Phi^{\prime}\left(X_{t}^{n, x}\right) \frac{\partial}{\partial x} X_{t}^{n, x}\right] \\
& =E\left[t^{-1} \int_{0}^{t} D_{s} \Phi\left(X_{t}^{n, x}\right) \frac{\partial}{\partial x} X_{s}^{n, x} d s\right] \\
& =E\left[\Phi\left(X_{t}^{n, x}\right) t^{-1} \int_{0}^{t} \frac{\partial}{\partial x} X_{s}^{n, x} d B_{s}\right],
\end{aligned}
$$

where we have used the chain rule and the duality formula for the Malliavin derivative in the last equality.

Fix $\varphi \in C_{0}^{\infty}(U)$. Using the matrix notation, we will show that

$$
\int_{\mathbb{R}^{d}} \frac{\partial}{\partial x} \varphi(x) v(t, x) d x=-\int_{\mathbb{R}^{d}} \varphi(x) E\left[\Phi\left(X_{t}^{x}\right) t^{-1} \int_{0}^{t} \frac{\partial}{\partial x} X_{s}^{x} d B_{s}\right] d x .
$$

Indeed, by dominated convergence and Remark 4.11 we have

$$
\begin{aligned}
\int_{\mathbb{R}^{d}} \frac{\partial}{\partial x} \varphi(x) v(t, x) d x= & -\lim _{n \rightarrow \infty} \int_{\mathbb{R}^{d}} \varphi(x) E\left[\Phi\left(X_{t}^{n, x}\right) t^{-1} \int_{0}^{t} \frac{\partial}{\partial x} X_{s}^{n, x} d B_{s}\right] d x \\
= & -\lim _{n \rightarrow \infty} \int_{\mathbb{R}^{d}} \varphi(x) E\left[\left(\Phi\left(X_{t}^{n, x}\right)-\Phi\left(X_{t}^{x}\right)\right) t^{-1} \int_{0}^{t} \frac{\partial}{\partial x} X_{s}^{n, x} d B_{s}\right] d x \\
& -\lim _{n \rightarrow \infty} \int_{\mathbb{R}^{d}} \varphi(x) E\left[\Phi\left(X_{t}^{x}\right) t^{-1} \int_{0}^{t} \frac{\partial}{\partial x} X_{s}^{n, x} d B_{s}\right] d x \\
& \left.\left.=-\lim _{n \rightarrow \infty} i\right)_{n}-\lim _{n \rightarrow \infty} i i\right)_{n} .
\end{aligned}
$$

For the first term we have

$$
i)_{n} \leq \int_{\mathbb{R}^{d}}|\varphi(x)|\left\|\frac{\partial}{\partial x} \Phi\right\|_{\infty}\left\|X_{t}^{n, x}-X_{t}^{x}\right\|_{L^{2}\left(\Omega ; \mathbb{R}^{d}\right)} t^{-1 / 2}\left(\sup _{k \geq 1, s \in[0,1]} E\left[\left\|\frac{\partial}{\partial x} X_{s}^{k, x}\right\|_{\mathbb{R}^{d \times d}}^{2}\right]\right)^{1 / 2} d x,
$$

which goes to zero as $n$ tends to infinity by Remark 4.11 and Lebesque dominated convergence theorem. For the second term, ii $)_{n}$ since $X_{t}^{x}$ is Malliavin differentiable and $\Phi \in C_{b}^{2}\left(\mathbb{R}^{d}\right)$ we have by the Clark-Ocone formula in matrix notational form that (see e.g. [34])

$$
\Phi\left(X_{t}^{x}\right)=E\left[\Phi\left(X_{t}^{x}\right)\right]+\int_{0}^{t} E\left[D_{s} \Phi\left(X_{t}^{x}\right) \mid \mathcal{F}_{s}\right] d B_{s},
$$


so that

$$
\begin{aligned}
i i)_{n} & =\int_{\mathbb{R}^{d}} \varphi(x) E\left[\Phi\left(X_{t}^{x}\right) t^{-1} \int_{0}^{t} \frac{\partial}{\partial x} X_{s}^{n, x} d B_{s}\right] d x \\
& =t^{-1} \int_{\mathbb{R}^{d}} \varphi(x) E\left[\left(E\left[\Phi\left(X_{t}^{x}\right)\right]+\int_{0}^{t} E\left[D_{s} \Phi\left(X_{t}^{x}\right) \mid \mathcal{F}_{s}\right] d B_{s}\right) \int_{0}^{t} \frac{\partial}{\partial x} X_{s}^{n, x} d B_{s}\right] d x \\
& =t^{-1} \int_{0}^{t} \int_{\mathbb{R}^{d}} \varphi(x) E\left[D_{s} \Phi\left(X_{t}^{x}\right) \frac{\partial}{\partial x} X_{s}^{n, x}\right] d x d s .
\end{aligned}
$$

It is easily verified by Lemma 2.5 that $\varphi(\cdot) D_{s} \Phi\left(X_{\dot{t}}\right)=\varphi(\cdot) \Phi^{\prime}\left(X_{\dot{t}}\right) D_{s} X_{\dot{t}}$ lies in $L^{2}\left(\mathbb{R}^{d} \times \Omega ; \mathbb{R}^{d}\right)$ so that for each $s$, the function

$$
g_{n}(s)=\int_{\mathbb{R}^{d}} \varphi(x) E\left[D_{s} \Phi\left(X_{t}^{x}\right) \frac{\partial}{\partial x} X_{s}^{n, x}\right] d x
$$

converges to $\int_{\mathbb{R}^{d}} \varphi(x) E\left[D_{s} \Phi\left(X_{t}^{x}\right) \frac{\partial}{\partial x} X_{s}^{x}\right] d x$ by the weak convergence of $\frac{\partial}{\partial x} X_{s}^{n, x}$ (for a subsequence). Now

$$
\begin{aligned}
\left|g_{n}(s)\right| & \leq \int_{\mathbb{R}^{d}}|\varphi(x)|\left\|D_{s} \Phi\left(X_{t}^{x}\right)\right\|_{L^{2}\left(\Omega ; \mathbb{R}^{d}\right)}\left\|\frac{\partial}{\partial x} X_{s}^{n, x}\right\|_{L^{2}\left(\Omega ; \mathbb{R}^{d}\right)} d x \\
& \leq \sup _{y \in \mathbb{R}^{d}, u \leq t, k \in \mathbb{N}}\left\|D_{u} \Phi\left(X_{t}^{y}\right)\right\|_{L^{2}\left(\Omega ; \mathbb{R}^{d}\right)}\left\|\frac{\partial}{\partial x} X_{u}^{k, y}\right\|_{L^{2}\left(\Omega ; \mathbb{R}^{d}\right)} \int_{\mathbb{R}^{d}}|\varphi(x)| d x
\end{aligned}
$$

so that by Lebesgue dominated convergence theorem

$$
\left.\lim _{n \rightarrow \infty} i i\right)_{n}=t^{-1} \int_{0}^{t} \int_{\mathbb{R}^{d}} \varphi(x) E\left[D_{s} \Phi\left(X_{t}^{x}\right) \frac{\partial}{\partial x} X_{s}^{x}\right] d x d s .
$$

Reversing equations (2.56), (2.57) and (2.58) with $\frac{\partial}{\partial x} X_{s}^{x}$ in place of $\frac{\partial}{\partial x} X_{s}^{n, x}$ gives the result.

Remark 2.24 i) In the case of smooth coefficients b a similar proof based on the duality formula for Malliavin derivatives can be found in [14].

ii) By using relation (2.55) and the existence of a probability density of $X_{t}^{x}$ in the proof of Theorem 2.23 it can actually be shown that for merely bounded and measurable $\Phi$ we have

$$
\left(x \mapsto E\left[\Phi\left(X_{t}^{x}\right)\right]\right) \in W^{1,2}(U)
$$

for all $t$.

\subsubsection{Well-posedness of stochastic transport equations with singular coefficients}

In order to emphasize the applicability of the techniques and results of the previous sections to a variety of other problems in stochastic analysis, we present the following result on the problem of well-posedness of stochastic transport equations with singular coefficients, which is based on the estimate (2.30) in Proposition 2.7.

The stochastic transport equation is the following equation 


$$
\begin{cases}d_{t} u(t, x)+ & \left(b(t, x) \cdot \frac{\partial}{\partial x} u(t, x)\right) d t+\sum_{i=1}^{d} \frac{\partial}{\partial x_{i}} u(t, x) \circ d B_{t}^{i}=0 \\ u(0, x)= & u_{0}(x),\end{cases}
$$

where $b:[0,1] \times \mathbb{R}^{d} \rightarrow \mathbb{R}^{d}$ is a given vector field and $u_{0}: \mathbb{R}^{d} \rightarrow \mathbb{R}$ is a given initial data. The stochastic integration is understood in the Stratonovich sense.

By a differentiable, weak $L^{\infty}$-solution of the transport equation (2.59) we mean a stochastic process $u \in L^{\infty}\left(\Omega \times[0,1] \times \mathbb{R}^{d}\right)$ such that, for every $t$, the function $u(t, \cdot)$ is weakly differentiable a.s. with $\sup _{0 \leq s \leq 1, x \in \mathbb{R}^{d}} E\left[\left|\frac{\partial}{\partial x} u(s, x)\right|^{4}\right]<\infty$ and for every test function $\theta \in C_{0}^{\infty}\left(\mathbb{R}^{d}\right)$, the process $\int_{\mathbb{R}^{d}} \theta(x) u(t, x) d x$ has a continuous modification which is an $\mathcal{F}_{t^{-}}$-semi martingale and

$$
\begin{aligned}
\int_{\mathbb{R}^{d}} \theta(x) u(t, x) d x & =\int_{\mathbb{R}^{d}} \theta(x) u_{0}(x) d x \\
& -\int_{0}^{t} \int_{\mathbb{R}^{d}} \frac{\partial}{\partial x} u(s, x) \cdot b(s, x) \theta(x) d x d s \\
& +\sum_{i=1}^{d} \int_{0}^{t}\left(\int_{\mathbb{R}^{d}} u(s, x) \frac{\partial}{\partial x_{i}} \theta(x) d x\right) \circ d B_{s}^{i},
\end{aligned}
$$

where $\frac{\partial}{\partial x} u(t, x)$ is the weak derivative of $u(t, x)$ in the space-variable.

Theorem 2.25 Let $b$ be bounded and measurable, and let $u_{0} \in C_{b}^{1}\left(\mathbb{R}^{d}\right)$. Then there exists a unique differentiable, weak $L^{\infty}$-solution $u(t, x)$ to (2.59). Moreover, for fixed $t$ and $x$, this solution is Malliavin-differentiable.

Proof. See [32].

Remark 2.26 We point out that the corresponding deterministic transport equation of (2.59) is in general not well-posed - even if $b$ is continuous.

Finally, we conclude this section with a remark on two further potential applications of our approach.

Remark 2.27 i) Another interesting implication of our result that strong solutions of SDE's with merely measurable drift coefficients are Malliavin differentiable is that for all $0 \leq t \leq T$, $h \in L^{2}([0, T])$ the process

$$
\left\{X_{t}\left(\omega+u \int_{0}^{\cdot} h(s) d s\right)\right\}_{u \in \mathbb{R}}
$$

has a version which is absolutely continuous for almost all $\omega \in \Omega=C_{0}([0, T])$ (Wiener space), see e.g. [34]. By considering the "initial condition" $y=x+B_{t}(\omega)$ in the ODE

$$
\begin{aligned}
\frac{d}{d t} X_{t}^{y} & =b\left(t, X_{t}^{y}\right) \\
X_{0}^{y} & =y,
\end{aligned}
$$

relation (2.61) in connection with (2.25) actually gives an interesting "link" to the flow property of solutions of ODE's with discontinuous coefficients. This may be of use in perturbation 
problems of discontinuous ordinary differential equations and other applications. See e.g. [27] or [12].

ii) We also mention that our technique may be applied to examine strong solutions of

$$
d X_{t}=b\left(t, X_{t}\right) d t+d B_{t}^{Q}, X_{0}=x \in H,
$$

where $B_{t}^{Q}$ is a $Q$-cylindrical Brownian motion on a Hilbert space $H$ and $Q$ a positive symmetric trace class operator. Applications to certain classes of SPDE's are also conceivable. See [28]. We point out that equations of the type (2.62) are not accessible within the framework of the authors of [52], [49], [36], [21], [16] and [17]. For example, the construction method of the authors in [17] heavily rests on an estimate of Krylov [20], which has no extension to infinite dimensions.

\subsection{Appendix}

The following result which is due to [6, Theorem 1] provides a compactness criterion for subsets of $L^{2}\left(\mu ; \mathbb{R}^{d}\right)$ using Malliavin calculus.

Theorem 2.28 Let $\{(\Omega, \mathcal{A}, P) ; H\}$ be a Gaussian probability space, that is $(\Omega, \mathcal{A}, P)$ is a probability space and $H$ a separable closed subspace of Gaussian random variables of $L^{2}(\Omega)$, which generate the $\sigma$-field $\mathcal{A}$. Denote by $\mathbf{D}$ the derivative operator acting on elementary smooth random variables in the sense that

$$
\mathbf{D}\left(f\left(h_{1}, \ldots, h_{n}\right)\right)=\sum_{i=1}^{n} \partial_{i} f\left(h_{1}, \ldots, h_{n}\right) h_{i}, h_{i} \in H, f \in C_{b}^{\infty}\left(\mathbb{R}^{n}\right) .
$$

Further let $\mathbf{D}_{1,2}$ be the closure of the family of elementary smooth random variables with respect to the norm

$$
\|F\|_{1,2}:=\|F\|_{L^{2}(\Omega)}+\|\mathbf{D} F\|_{L^{2}(\Omega ; H)} .
$$

Assume that $C$ is a self-adjoint compact operator on $H$ with dense image. Then for any $c>0$ the set

$$
\mathcal{G}=\left\{G \in \mathbf{D}_{1,2}:\|G\|_{L^{2}(\Omega)}+\left\|C^{-1} \mathbf{D} G\right\|_{L^{2}(\Omega ; H)} \leq c\right\}
$$

is relatively compact in $L^{2}(\Omega)$.

In order to formulate compactness criteria useful for our purposes, we need the following technical result which also can be found in [6].

Lemma 2.29 Let $v_{s}, s \geq 0$ be the Haar basis of $L^{2}([0,1])$. For any $0<\alpha<1 / 2$ define the operator $A_{\alpha}$ on $L^{2}([0,1])$ by

$$
A_{\alpha} v_{s}=2^{k \alpha} v_{s}, \text { if } s=2^{k}+j
$$

for $k \geq 0,0 \leq j \leq 2^{k}$ and

$$
A_{\alpha} 1=1 .
$$

Then for all $\beta$ with $\alpha<\beta<(1 / 2)$, there exists a constant $c_{1}$ such that

$$
\left\|A_{\alpha} f\right\| \leq c_{1}\left\{\|f\|_{L^{2}([0,1])}+\left(\int_{0}^{1} \int_{0}^{1} \frac{\left|f(t)-f\left(t^{\prime}\right)\right|^{2}}{\left|t-t^{\prime}\right|^{1+2 \beta}} d t d t^{\prime}\right)^{1 / 2}\right\} .
$$


A direct consequence of Theorem 2.28 and Lemma 2.29 is now the following compactness criteria which is essential for the proof of Corollary 2.6:

Corollary 2.30 Let a sequence of $\mathcal{F}_{1}$-measurable random variables $X_{n} \in \mathbb{D}_{1,2}, n=1,2 \ldots$, be such that there exist constants $\alpha>0$ and $C>0$ with

$$
\begin{gathered}
\sup _{n} E\left[\left|X_{n}\right|^{2}\right] \leq C, \\
\sup _{n} E\left[\left\|D_{t} X_{n}-D_{t^{\prime}} X_{n}\right\|^{2}\right] \leq C\left|t-t^{\prime}\right|^{\alpha}
\end{gathered}
$$

for $0 \leq t^{\prime} \leq t \leq 1$ and

$$
\sup _{n} \sup _{0 \leq t \leq 1} E\left[\left\|D_{t} X_{n}\right\|^{2}\right] \leq C .
$$

Then the sequence $X_{n}, n=1,2 \ldots$, is relatively compact in $L^{2}(\Omega)$. 


\section{References}

[1] A.N. Borodin, P. Salminen, Handbook of Brownian Motion \&3 Facts and Formulae. Second edition. Birkhauser Verlag, (2002).

[2] A. Bressan, W. Sheng, On discontinuous differential equations. In: Differential Inclusions and Optimal Control, Lect. Notes Nonlin. Anal., 2 (1988), 73-87.

[3] R. Buckdahn, Y. Ouknine, M. Quincampoix, On limiting values of stochastic differential equations with small noise intensity tending to zero. Bull. Sci. math. 133 (3) (2009), 229-237.

[4] T-S. Chiang, C-H. Hwang, On the non-uniqueness of the limit points of diffusions with a small parameter. Stochastics 10 (2) (1983), 149-153.

[5] T-S. Chiang, S-J. Sheu, Large deviation of diffusion processes with discontinuous drift and their occupation times. Annals of Probability 28 (1) (2000), 140-165.

[6] G. Da Prato, P. Malliavin, D. Nualart, Compact families of Wiener functionals. C. R. Acad. Sci. Paris, Sr. I 315 (1992), 1287-1291.

[7] A. M. Davie, Uniqueness of solutions of stochastic differential equations. Int. Math. Res. 24, Article ID rnm 124, (2007), 26 P.]

[8] G. Di Nunno, B. Øksendal, F. Proske, Malliavin Calculus for Lévy Processes with Applications to Finance. Springer (2008).

[9] N. Eisenbaum, Integration with respect to local time, Potential Analysis 13 (2000), 303328.

[10] Elworthy, K. D., Li, X. -M., Formulae for the derivatives of heat semigroups, J. Funct. Anal. 125 , 252286 (1994)

[11] S. Fang, T. Zhang: A class of stochastic differential equations with non-Lipschitzian coefficients: pathwise uniqueness and no explosion. C. R. Acad. Sci. Paris, Ser. I 337 (2003).

[12] E. Fedrizzi and F. Flandoli, Hölder Flow and Differentiability for SDEs with Nonregular Drift, (2010), preprint.

[13] F. Flandoli, Remarks on uniqueness and strong solutions to deterministic and stochastic differential equations. Metrika 69 (2009) 101-123.

[14] Fourni, E., Lasry, J-M., Lebuchoux, J., Lions, P-L. and Touzi, N., Applications of Malliavin Calculus to Monte Carlo methods in finance. Finance Stoch., 3(4), 391-412 (1999)

[15] R. Ghomrasni, O. Menoukeu Pamen, An approximation of the generalized covariation process. Submitted (2010).

[16] I. Gyöngy, N. V. Krylov, Existence of strong solutions for Itô's stochastic equations via approximations. Probab. Theory Relat. Fields, 105 (1996), 143-158. 
[17] I. Gyöngy, T. Martinez, On stochastic differential equations with locally unbounded drift. Czechoslovak Mathematical Journal, 51 (4) (2001), 763-783.

[18] T. Hida, H.-H. Kuo, J. Potthoff, L. Streit, White Noise: An Infinite Dimensional Calculus. Kluwer Academic, (1993).

[19] G. Kallianpur, J. Xiong, Stochastic Differential Equations in Infinite Dimensional Spaces. IMS Lecture Notes Monograph Series (1995).

[20] N. V. Krylov, Estimates of the maximum of the solution of a parabolic equation and estimates of the distribution of a semimartingale. (Russian) Mat. Sb. (N.S.) 130 (172) (1986), 207-221.

[21] N.V. Krylov, M. Röckner, Strong solutions of stochastic equations with singular time dependent drift. Prob. Theory Rel. Fields 131 (2) (2005), 154-196.

[22] Kunita, H.: Stochastic Flows and Stochastic Differential Equations. Cambridge University Press (1990).

[23] H.-H. Kuo, White Noise Distribution Theory. Prob. and Soch. Series, Boca Raton, FL: CRC Press, (1996).

[24] A. Lanconelli, F. Proske, On explicit strong solutions of Itô-SDE's and the Donsker delta function of a diffusion. Infin. Dimen. Anal. Quant. Prob. related Topics, 7 (3) (2004).

[25] P. Malliavin, Stochastic calculus of variations and hypoelliptic operators. In: Proc. Inter. Symp. on Stoch. Diff. Equations, Kyoto 1976, Wiley, (1978), 195-263.

[26] P. Malliavin, Stochastic Analysis. Springer (1997)

[27] O. Menoukeu-Pamen, T. Meyer-Brandis, F. Proske, A Gel'fand triple approach to the small noise problem for discontinuous ODE's. Preprint, University of Oslo (2010).

[28] O. Menoukeu-Pamen, T. Meyer-Brandis, T. Nilssen, F. Proske, T. Zhang, Malliavin smoothness of SPDE's with irregular drift coefficients. In preparation.

[29] T. Meyer-Brandis, F. Proske, On the existence and explicit representability of strong solutions of Lévy noise driven SDE's. Communications in Mathematical Sciences, 4 (1) (2006).

[30] T. Meyer-Brandis, F. Proske, Explicit representation of strong solutions of SDE's driven by infinite dimensional Lévy processes. Journal of Theoretical Probability, to appear(2009).

[31] T. Meyer-Brandis, F. Proske, Construction of strong solutions of SDE's via Malliavin calculus. Journal of Funct. Anal. 258 (2010), 3922-3953.

[32] Mohammed, S.E.A., Nilssen, T. Proske, F.: Sobolev differentiable stochastic flows of SDE's with measurable drift and applications. arXiv: 1204.3867 (2012).

[33] I. Mitoma, Tightness of Probabilities on $C\left([0,1], S^{\prime}\right)$ and $D\left([0,1], S^{\prime}\right)$. Annals of Probability 11 (4) (1983), 989-999. 
[34] D. Nualart, The Malliavin Calculus and Related Topics. Springer (1995).

[35] N. Obata, White Noise Calculus and Fock Space. LNM 1577, Springer (1994).

[36] N. I. Portenko, Generalized Diffusion Processes. Transl. Math. Monographs, 83, Amer. Math. Soc., Providence, R. I. (1990).

[37] J. Potthoff, L. Streit, A characterization of Hida distributions. Journal of Funct. Anal. 101 (1991) 212-229.

[38] F. Proske, Stochastic differential equations- some new ideas. Stochastics, 79 (2007), 563-600.]

[39] D. Revuz, M. Yor, Continuous Martingales and Brownian Motion. Third edition, Springer, 2004.

[40] F. Russo and P. Vallois, Forward, backward and symmetric stochastic integration. Probab. Theory Related Fields 97 (1993), 403-421.

[41] F. Russo and P. Vallois, The generalized covariation process and Itô formula. Stochastic Process. Appl 59 (1995), 81-104.

[42] F. Russo and P. Vallois, Itô formula for $C^{1}$-functions of semimartingales. Probab. Theory Related Fields 104 (1996), 27-41.

[43] F. Russo and P. Vallois, Stochastic calculus with respect to continuous finite quadratic variation processes. Stochastics Stochastics Rep. 70 (2000), 1-40.

[44] F. Russo and P. Vallois, Elements of Stochastic Calculus via Regularization. Sém. Prob. XL, Lect. Notes in Math. 1899, Springer, Berlin, (2007), 147-185.

[45] F. Russo, P. Vallois and J. Wolf, A generalized class of Lyons-Zheng processes. Bernoulli 7 (2) (2001), 363-379.

[46] W. Rzymowski, Existence of Solutions for a Class of Discontinuous Differential Equations in $\mathbb{R}^{n}$. J. Math. Anal. Appl. 233 (1999), 634-643.

[47] P. Salminen, M. Yor, Property of perpetual integral functionals of Brownian motion with drift. Ann. Inst. H. Poincare Probab. Statist. 41 (3) (2005), 335-347.

[48] S.R.S. Varadhan, Large deviations and applications. CBMS-NSF Regional Conference Series in Applied Mathematics, 46. Society for Industrial and Applied Mathematics (SIAM), Philadelphia, PA, (1984).

[49] A.Y. Veretennikov, On the strong solutions of stochastic differential equations. Theory Probab. Appl. 24 (1979), 354-366.

[50] A.Y. Veretennikov, On the strong solutions and explicit formulas for solutions of stochastic differential equations. Math. USSR Sbornik. 39(3) (1981), 387-403.

[51] A.Y. Veretennikov, Approximation of ordinary differential equations by stochastic ones. Mat. Zametki 33 (6) (1983), 929-932 (in Russian).

[52] A.K. Zvonkin, A transformation of the state space of a diffusion process that removes the drift. Math.USSR (Sbornik) 22 (1974), 129-149. 


\title{
3 Sobolev Differentiable Stochastic Flows for SDE's with Singluar Coefficients: Applications to the Transport Equation
}

\author{
Salah Mohammed, Torstein Nilssen and Frank Proske
}

Preprint

\begin{abstract}
In this paper, we establish the existence of a stochastic flow of Sobolev diffeomorphisms

$$
\mathbb{R}^{d} \ni x \longmapsto \phi_{s, t}(x) \in \mathbb{R}^{d}, s, t \in \mathbb{R},
$$
\end{abstract}

for a stochastic differential equation (SDE) of the form

$$
d X_{t}=b\left(t, X_{t}\right) d t+d B_{t}, s, t \in \mathbb{R}, \quad X_{s}=x \in \mathbb{R}^{d} .
$$

The above SDE is driven by a bounded measurable drift coefficient $b: \mathbb{R} \times \mathbb{R}^{d} \rightarrow \mathbb{R}^{d}$ and a $d$-dimensional Brownian motion $B$. More specifically, we show that the stochastic flow $\phi_{s, t}(\cdot)$ of the SDE lives in the space $L^{2}\left(\Omega ; W^{1, p}\left(\mathbb{R}^{d}, w\right)\right)$ for all $s, t$ and all $p>1$, where $W^{1, p}\left(\mathbb{R}^{d}, w\right)$ denotes a weighted Sobolev space with weight $w$ possessing a $p$-th moment with respect to Lebesgue measure on $\mathbb{R}^{d}$. This result is counter-intuitive, since the dominant 'culture' in stochastic (and deterministic) dynamical systems is that the flow 'inherits' its spatial regularity from the driving vector fields.

The spatial regularity of the stochastic flow yields existence and uniqueness of a Sobolev differentiable weak solution of the (Stratonovich) stochastic transport equation

$$
\begin{cases}d_{t} u(t, x)+ & (b(t, x) \cdot D u(t, x)) d t+\sum_{i=1}^{d} e_{i} \cdot D u(t, x) \circ d B_{t}^{i}=0, \\ u(0, x)= & u_{0}(x)\end{cases}
$$

where $b$ is bounded and measurable, $u_{0}$ is $C_{b}^{1}$ and $\left\{e_{i}\right\}_{i=1}^{d}$ a basis for $\mathbb{R}^{d}$. It is well-known that the deterministic counter part of the above equation does not in general have a solution.

Using stochastic perturbations and our analysis of the above SDE, we establish a deterministic flow of Sobolev diffeomorphisms for classical one-dimensional (deterministic) ODE's driven by discontinuous vector fields. Furthermore, and as a corollary of the latter result, we construct a Sobolev stochastic flow of diffeomorphisms for one-dimensional SDE's driven by discontinuous diffusion coefficients. 


\subsection{Introduction}

In this article we analyze the spatial regularity in the initial condition $x \in \mathbb{R}^{d}$ for strong solutions $X^{x}$ to the $d$-dimensional stochastic differential equation (SDE)

$$
X_{t}^{s, x}=x+\int_{s}^{t} b\left(u, X_{u}^{s, x}\right) d u+B_{t}-B_{s}, \quad s, t \in \mathbb{R} .
$$

In the above SDE, the drift coefficient $b: \mathbb{R} \times \mathbb{R}^{d} \longrightarrow \mathbb{R}^{d}$ is only Borel measurable and bounded, and the equation is driven by standard Brownian motion $B$. in $\mathbb{R}^{d}$.

More specifically, we construct a two-parameter pathwise Sobolev differentiable stochastic flow

$$
\mathbb{R} \times \mathbb{R} \times \mathbb{R}^{d} \ni(s, t, x) \longmapsto \phi_{s, t}(x) \in \mathbb{R}^{d}
$$

for the SDE (3.1) such that each flow map

$$
\mathbb{R}^{d} \ni x \longmapsto \phi_{s, t}(x) \in \mathbb{R}^{d}
$$

is a Sobolev diffeomorphism in the sense that

$$
\phi_{s, t}(\cdot) \text { and } \phi_{s, t}^{-1}(\cdot) \in L^{2}\left(\Omega, W^{1, p}\left(\mathbb{R}^{d} ; w\right)\right)
$$

for all $s, t \in \mathbb{R}$, all $p>1$. In (3.2) above, $W^{1, p}\left(\mathbb{R}^{d}, w\right)$ denotes a weighted Sobolev space of mappings $\mathbb{R}^{d} \rightarrow \mathbb{R}^{d}$ with any measurable weight function $w: \mathbb{R}^{d} \rightarrow[0, \infty)$ satisfying the integrability requirement

$$
\int_{\mathbb{R}^{d}}\left(1+|x|^{p}\right) w(x) d x<\infty .
$$

In particular, $\phi_{s, t}(\cdot)$ is locally $\alpha$-Hölder continuous for all $\alpha<1$. When the SDE (3.1) is autonomous, we show further that the stochastic flow corresponds to a Sobolev differentiable perfect cocycle on $\mathbb{R}^{d}$. For precise statements of the above results, see Theorem 3.3 and Corollary 3.5 in the next section.

In this article we offer a novel approach for constructing a Sobolev differentiable stochastic flow for the SDE (1). Our approach is based on Malliavin calculus ideas coupled with new probabilistic estimates on the spatial weak derivatives of solutions of the SDE. A unique (pleasantly surprising) feature of these estimates is that they do not depend on the spatial regularity of the drift coefficient $b$. Needless to say, the existence of differentiable flows for SDE's with measurable drifts is counter-intuitive: The dominant 'culture' in stochastic (and deterministic) dynamical systems is that the flow 'inherits' its spatial regularity from the driving vector fields. Furthermore, in the stochastic setting, the stochastic flow is in general even a little 'rougher' in the space variable than the driving vector fields. (cf. [22], [28]).

The existence of a Sobolev differentiable stochastic flow for the SDE (1) is exploited (Section 3) to obtain a unique weak solution $u(t, x)$ of the (Stratonovich) stochastic transport equation

$$
\begin{cases}d_{t} u(t, x)+ & (b(t, x) \cdot D u(t, x)) d t+\sum_{i=1}^{d} e_{i} \cdot D u(t, x) \circ d B_{t}^{i}=0 \\ u(0, x)= & u_{0}(x)\end{cases}
$$

when $b$ is just bounded and measurable, $u_{0} \in C_{b}^{1}\left(\mathbb{R}^{d}\right)$, and $\left\{e_{i}\right\}_{i=1}^{d}$ a basis for $\mathbb{R}^{d}$. This result is surprising since the corresponding deterministic transport equation is in general ill-posed. 
Cf. [1], [8]. We also note that our result holds without the existence of the divergence of $b$; and furthermore, our solutions are spatially (and also Malliavin) Sobolev differentiable (cf. [14]).

In Section 4, we apply the ideas of Section 2 to show the existence of a family of solutions $\tilde{X}_{t}^{x}$ of the one-dimensional ODE

$$
\frac{d \tilde{X}_{t}}{d t}=b\left(\tilde{X}_{t}\right), \quad t \in \mathbb{R}, \quad \tilde{X}_{0}=x \in \mathbb{R}
$$

which are locally of class $W^{1,2}$ in $x$ (Theorem 3.27, Section 4). This result is obtained under the requirement that the coefficient $b$ is monotone decreasing and is either bounded above or below. The proof of the result uses a stochastic perturbation argument via small Brownian noise coupled with local time techniques. As far as we know, it appears that the above result is new. Furthermore, solutions to the $\operatorname{ODE}$ (3.5) generate a one-parameter group of $W^{1,2}$ diffeomorphisms of $\mathbb{R}$ onto itself. As a consequence of the above result, we construct a $W^{1,2}$ perfect cocycle of diffeomorphisms for solutions of the one-dimensional Stratonovich SDE:

$$
d X_{t}^{x}=b\left(X_{t}^{x}\right) \circ d B_{t}, t \in \mathbb{R}, \quad X_{0}^{x}=x \in \mathbb{R} .
$$

It is surprising that such regularity of the flow is feasible despite the inherent discontinuities in the driving vector field of in the ODE (3.5) and the SDE (3.6). SDE's with discontinuous coefficients and driven by Brownian motion (or more general noise) have been an important area of study in stochastic analysis and other related branches of mathematics. Important applications of this class of SDE's pertain to the modeling of the dynamics of interacting particles in statistical mechanics and the description of a variety of other random phenomena in areas such as biology or engineering. See e.g. [33] or [23] and the references therein.

Using estimates of solutions of parabolic PDE's and the Yamada-Watanabe principle, the existence of a global unique strong solution to the SDE (3.1) was first established by A.K. Zvonkin [41] in the 1-dimensional case, when $b$ is bounded and measurable. The latter work is a significant development in the theory of SDE's. Subsequently, the result was generalized by A.Y. Veretennikov [39] to the multi-dimensional case. More recently, N.V. Krylov and M. Röckner employed local integrability criteria on the drift coefficient $b$ to obtain unique strong solutions of (3.1) by using an argument of N. I. Portenko [33]. An alternative approach, which doesn't rely on a pathwise uniqueness argument and which also yields the Malliavin differentiability of solutions to (3.1) was recently developed in [27], [26]. We also refer to the recent article [5] for an extension of the previous results to a Hilbert space setting. In [5], the authors employ techniques based on solutions of infinite-dimensional Kolmogorov equations.

Another important issue in the study of SDE's with (bounded) measurable coefficients is the regularity of their solutions with respect to the initial data and the existence of stochastic flows. See [22], [28] for more information on the existence and regularity of stochastic flows for SDE's, and [29], [30] in the case of stochastic differential systems with memory. Using the method of stochastic characteristics, stochastic flows may be employed to prove uniqueness of solutions of stochastic transport equations under weak regularity hypotheses on the drift coefficient $b$. See for example [14], where the authors use estimates of solutions of backward Kolmogorov equations to show the existence of a stochastic flow of diffeomorphisms with $\alpha^{\prime}$-Hölder continuous derivatives for $\alpha^{\prime}<\alpha$, where $b \in C\left([0,1] ; C_{b}^{\alpha}\left(\mathbb{R}^{d}\right)\right)$, and $C_{b}^{\alpha}\left(\mathbb{R}^{d}\right)$ is the space of bounded $\alpha$-Hölder continuous functions. A similar result also holds true, when $b \in L^{q}\left([0,1] ; L^{p}\left(\mathbb{R}^{d}\right)\right)$ for $p, q$ such that $p \geq 2, q>2, \frac{d}{p}+\frac{2}{q}<1$. See [12]. Here the authors 
construct, for any $\alpha \in(0,1)$, a stochastic flow of $\alpha$-Hölder continuous homeomorphisms for the SDE (3.1). Furthermore, it is shown in [12] that the map

$$
\mathbb{R}^{d} \ni x \longmapsto X^{x} \in L^{p}\left([0,1] \times \Omega ; \mathbb{R}^{d}\right)
$$

is differentiable in the $L^{p}(\Omega)$-sense for every $p \geq 2$.

The approach used in [12] is based on a Zvonkin-type transformation [41] and estimates of solutions of an associated backward parabolic PDE. We also mention the recent related works [11], [10] and [2]. For an overview of this topic the reader may also consult the book $[8] .{ }^{1}$ In this connection, it should be noted that our method for constructing a stochastic flow for the SDE (3.1) is heavily dependent on Malliavin calculus ideas together with some difficult probabilistic estimates (cf. [26]).

Our paper is organized as follows: In Section, 2 we introduce basic definitions and notations and provide some auxiliary results that are needed to prove the existence of a Sobolev differentiable stochastic flow for the SDE (3.1). See Theorem 3.3 and Corollary 3.5 in Section 2. We also briefly discuss a specific extension of this result to SDE's with multiplicative noise. In Section 3 we give an application of our approach to the construction of a unique Sobolev differentiable solution to the (Stratonovich) stochastic transport equation (3.27) . Ideas developed in Section 2 are used in Section 4 to show the existence and regularity of a deterministic flow for the one-dimensional ODE (3.5), and a perfect cocycle for the one-dimensional SDE $(3.6)$.

\subsection{Existence of a Sobolev Differentiable Stochastic Flow}

Throughout this paper we denote by $B_{t}=\left(B_{t}^{(1)}, \ldots, B_{t}^{(d)}\right), t \in \mathbb{R}, d$-dimensional Brownian motion on the complete Wiener space $(\Omega, \mathcal{F}, \mu)$ where $\Omega:=C\left(\mathbb{R} ; \mathbb{R}^{d}\right)$ is given the compact open topology and $\mathcal{F}$ is its $\mu$-completed Borel $\sigma$-field with respect to Wiener measure $\mu$.

In order to describe the cocycle associated with the stochastic flow of our SDE, we define the $\mu$-preserving (ergodic) Wiener shift $\theta(t, \cdot): \Omega \rightarrow \Omega$ by

$$
\theta(t, \omega)(s):=\omega(t+s)-\omega(t), \omega \in \Omega, t, s \in \mathbb{R} .
$$

The Brownian motion is then a perfect helix with respect to $\theta$ : That is

$$
B_{t_{1}+t_{2}}(\omega)-B_{t_{1}}(\omega)=B_{t_{2}}\left(\theta\left(t_{1}, \omega\right)\right)
$$

for all $t_{1}, t_{2} \in \mathbb{R}$ and all $\omega \in \Omega$. The above helix property is a convenient pathwise expression of the fact that Brownian motion $B$ has stationary ergodic increments.

Our main focus of study in this section is the $d$-dimensional SDE

$$
X_{t}^{s, x}=x+\int_{s}^{t} b\left(u, X_{u}^{s, x}\right) d u+B_{t}-B_{s}, \quad s, t \in \mathbb{R}, x \in \mathbb{R}^{d},
$$

where the drift coefficient $b: \mathbb{R} \times \mathbb{R}^{d} \longrightarrow \mathbb{R}^{d}$ is a bounded Borel measurable function.

It is known that the above SDE has a unique strong global solution $X^{s, x}$ for each $x \in \mathbb{R}^{d}$ ( [39] or [26], [27]).

Here, we will establish the existence of a Sobolev-differentiable stochastic flow of diffeomorphisms for the SDE (3.7).

\footnotetext{
${ }^{1}$ After completing the preparation of this article, personal communication with F. Flandoli indicated work in preparation with E. Fedrizzi [13] on similar issues regarding the regularity of stochastic flows for SDE's, using a different approach.
} 
Definition 3.1 A map $\mathbb{R} \times \mathbb{R} \times \mathbb{R}^{d} \ni(s, t, x, \omega) \longmapsto \phi_{s, t}(x, \omega) \in \mathbb{R}^{d}$ is a stochastic flow of homeomorphisms for the $S D E$ (3.7) if there exists a universal set $\Omega^{*} \in \mathcal{F}$ of full Wiener measure such that for all $\omega \in \Omega^{*}$, the following statements are true:

(i) For any $x \in \mathbb{R}^{d}$, the process $\phi_{s, t}(x, \omega), s, t \in \mathbb{R}$, is a strong global solution to the $S D E$ (3.7).

(ii) $\phi_{s, t}(x, \omega)$ is continuous in $(s, t, x) \in \mathbb{R} \times \mathbb{R} \times \mathbb{R}^{d}$.

(iii) $\phi_{s, t}(\cdot, \omega)=\phi_{u, t}(\cdot, \omega) \circ \phi_{s, u}(\cdot, \omega)$ for all $s, u, t \in \mathbb{R}$.

(iv) $\phi_{s, s}(x, \omega)=x$ for all $x \in \mathbb{R}^{d}$ and $s \in \mathbb{R}$.

(v) $\phi_{s, t}(\cdot, \omega): \mathbb{R}^{d} \rightarrow \mathbb{R}^{d}$ are homeomorphisms for all $s, t \in \mathbb{R}$.

A stochastic flow $\phi_{s, t}(\cdot, \omega)$ of homeomorphisms is said to be Sobolev-differentiable if for all $s, t \in \mathbb{R}$, the maps $\phi_{s, t}(\cdot, \omega)$ and $\phi_{s, t}^{-1}(\cdot, \omega)$ are Sobolev-differentiable in the sense described below.

From now on we use $|\cdot|$ to denote the norm of a vector in $\mathbb{R}^{d}$ or a matrix in $\mathbb{R}^{d \times d}$.

In order to prove the existence of a Sobolev differentiable flow for the SDE (3.7), we need to introduce a suitable class of weighted Sobolev spaces. Fix $p \in(1, \infty)$ and let $w: \mathbb{R}^{d} \longrightarrow(0, \infty)$ be a Borel measurable function satisfying

$$
\int_{\mathbb{R}^{d}}\left(1+|x|^{p}\right) w(x) d x<\infty .
$$

Let $L^{p}\left(\mathbb{R}^{d}, w\right)$ denote the Banach space of all Borel measurable functions $u=\left(u_{1}, \ldots, u_{d}\right)$ : $\mathbb{R}^{d} \longrightarrow \mathbb{R}^{d}$ such that

$$
\int_{\mathbb{R}^{d}}|u(x)|^{p} w(x) d x<\infty,
$$

and equipped with the norm

$$
\|u\|_{L^{p}\left(\mathbb{R}^{d}, w\right)}:=\left[\int_{\mathbb{R}^{d}}|u(x)|^{p} w(x) d x\right]^{1 / p} .
$$

Furthermore, denote by $W^{1, p}\left(\mathbb{R}^{d}, w\right)$ the linear space of functions $u \in L^{p}\left(\mathbb{R}^{d}, w\right)$ with weak partial derivatives $D_{j} u \in L^{p}\left(\mathbb{R}^{d}, w\right)$ for $j=1, \ldots, d$. We equip this space with the complete norm

$$
\|u\|_{1, p, w}:=\|u\|_{L^{p}\left(\mathbb{R}^{d}, w\right)}+\sum_{i, j=1}^{d}\left\|D_{j} u_{i}\right\|_{L^{p}\left(\mathbb{R}^{d}, w\right)} .
$$

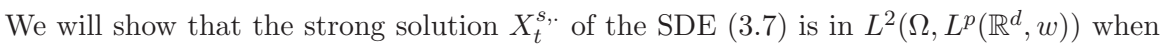
$p>1$ (see Corollary 3.13). In fact, the SDE (3.7) implies the following estimate:

$$
\left|X_{t}^{s, x}\right|^{p} \leq c_{p}\left(|x|^{p}+|t-s|^{p}\|b\|_{\infty}^{p}+\left|B_{t}-B_{s}\right|^{p}\right) .
$$

for all $s, t \in \mathbb{R}, x \in \mathbb{R}^{d}$.

On the other hand, it is easy to see that the solutions $X_{t}^{s, .}$ of SDE (3.7) are in general not in $L^{p}\left(\mathbb{R}^{d}, d x\right)$ with respect to Lebesgue measure $d x$ on $\mathbb{R}^{d}$ : Just consider the special trivial case $b \equiv 0$. This implies that solutions of the SDE (3.7) (if they exist) may not belong to the Sobolev space $W^{1, p}\left(\mathbb{R}^{d}, d x\right), p>1$. However, we will show that such solutions do indeed belong to the weighted Sobolev spaces $W^{1, p}\left(\mathbb{R}^{d}, w\right)$ for $p \geq 1$. 
Remark 3.2 (i) Let $w: \mathbb{R}^{d} \longrightarrow(0, \infty)$ be a weight function in Muckenhoupt's $A_{p}$-class $(1<p<\infty)$, that is a locally (Lebesgue) integrable function on $\mathbb{R}^{d}$ such that

$$
\sup \left(\frac{1}{\lambda_{d}(B)} \int_{B} w(x) d x\right)\left(\frac{1}{\lambda_{d}(B)} \int_{B}(w(x))^{1 /(1-p)} d x\right)^{p-1}=: c_{w, p}<\infty
$$

where the supremum is taken over all balls $B$ in $\mathbb{R}^{d}$ and $\lambda_{d}$ is Lebesgue measure on $\mathbb{R}^{d}$. For example the function $w(x)=|x|^{\gamma}$ is an $A_{p}$-weight iff $-d<\gamma<d(p-1)$. Other examples of weights are given by positive superharmonic functions. See e.g. [18] and [21] and the references therein. Denote by $H^{1, p}\left(\mathbb{R}^{d}, w\right)$ the completion of $C^{\infty}\left(\mathbb{R}^{d}\right)$ with respect to the norm $\|\cdot\|_{1, p, w}$ in (3.10). If $w$ is a $A_{p}-$ weight, then we have

$$
W^{1, p}\left(\mathbb{R}^{d}, w\right)=H^{1, p}\left(\mathbb{R}^{d}, w\right)
$$

for all $1<p<\infty$. See e.g. [18].

(ii) Let $p_{0}=\inf \left\{q>1: w\right.$ is a $A_{q}-$ weight $\}$ and let $u \in W^{1, p}\left(\mathbb{R}^{d}, w\right)$. If $p_{0}<p / d$, then $u$ is locally Hölder continuous with any exponent $\alpha$ such that $0<\alpha<1-d p_{0} / p$.

We now state our main result in this section which gives the existence of a Sobolev differentiable stochastic flow for the SDE (3.7).

Theorem 3.3 In the SDE (3.7), assume that the drift coefficient $b$ is Borel-measurable and bounded. Then the SDE (3.7) has a Sobolev differentiable stochastic flow $\phi_{s, t}: \mathbb{R}^{d} \rightarrow \mathbb{R}^{d}, s, t \in$ $\mathbb{R}$ : That is

$$
\phi_{s, t}(\cdot) \text { and } \phi_{s, t}^{-1}(\cdot) \in L^{2}\left(\Omega, W^{1, p}\left(\mathbb{R}^{d}, w\right)\right)
$$

for all $s, t \in \mathbb{R}$ and all $p>1$.

Remark 3.4 If $w$ is a $A_{p}$-weight then it follows from Remark 3.2 (ii) that a version of $\phi_{s, t}(\cdot)$ is locally Hölder continuous for all $0<\alpha<1$ and all $s, t$.

The following corollary is a consequence of Theorem 3.3 and the helix property of the Brownian motion.

Corollary 3.5 Consider the autonomous SDE

$$
X_{t}^{s, x}=x+\int_{s}^{t} b\left(X_{u}^{s, x}\right) d u+B_{t}-B_{s}, \quad s, t \in \mathbb{R},
$$

with bounded Borel-measurable drift $b: \mathbb{R}^{d} \rightarrow \mathbb{R}^{d}$. Then the stochastic flow of the SDE (3.11) has a version which generates a perfect Sobolev-differentiable cocycle $\left(\phi_{0, t}, \theta(t, \cdot)\right)$ where $\theta(t, \cdot): \Omega \rightarrow \Omega$ is the $\mu$-preserving Wiener shift. More specifically, the following perfect cocycle property holds for all $\omega \in \Omega$ and all $t_{1}, t_{2} \in \mathbb{R}$ :

$$
\phi_{0, t_{1}+t_{2}}(\cdot, \omega)=\phi_{0, t_{2}}\left(\cdot, \theta\left(t_{1}, \omega\right)\right) \circ \phi_{0, t_{1}}(\cdot, \omega)
$$

We will prove Theorem 3.3 through a sequence of lemmas and propositions. We begin by stating our main proposition: 
Proposition 3.6 Let $b: \mathbb{R} \times \mathbb{R}^{d} \rightarrow \mathbb{R}^{d}$ be bounded and measurable. Let $U$ be an open and bounded subset of $\mathbb{R}^{d}$. For each $t \in \mathbb{R}$ and $p>1$ we have

$$
X_{t} \in L^{2}\left(\Omega ; W^{1, p}(U)\right)
$$

We will prove Proposition 3.6 using two steps. In the first step, we show that for a bounded smooth function $b:[0,1] \times \mathbb{R}^{d} \rightarrow \mathbb{R}^{d}$ with compact support, it is possible to estimate the norm of $X_{t}$ in $L^{2}\left(\Omega, W^{1, p}(U)\right)$ independently of the size of $b^{\prime}$, with the estimate depending only on $\|b\|_{\infty}$. To do this we use the same technique as introduced in [26].

In the second step, we will approximate our bounded measurable coefficient $b$ by a sequence $\left\{b_{n}\right\}_{n=1}^{\infty}$ of smooth compactly supported functions as in step 1 . We then show that the corresponding sequence $X_{t}^{n, \cdot}$ of solutions is relatively compact in $L^{2}(\Omega)$ when integrated against a test function on $\mathbb{R}^{d}$. By step 1 we use weak compactness of the above sequence in $L^{2}\left(\Omega, W^{1, p}(U)\right)$ to conclude that the limit point $X_{t}$ of the above sequence must also lie in this space.

We now turn to the first step of our procedure. Note that if $b$ is a compactly supported smooth function, the corresponding solution of the SDE (3.1) is (strongly) differentiable with respect to $x$, and the first order spatial Jacobian $\frac{\partial}{\partial x} X_{t}^{x}$ satisfies the linearized random ODE

$$
\left\{\begin{array}{l}
d \frac{\partial}{\partial x} X_{t}^{x}=b^{\prime}\left(t, X_{t}^{x}\right) \frac{\partial}{\partial x} X_{t}^{x} d t \\
\frac{\partial}{\partial x} X_{0}^{x}=\mathcal{I}_{d}
\end{array}\right.
$$

where $\mathcal{I}_{d}$ is the $d \times d$ identity matrix and $b^{\prime}(t, x)=\left(\frac{\partial}{\partial x_{i}} b^{(j)}(t, x)\right)_{1 \leq i, j \leq d}$ denotes the spatial Jacobian derivative of $b$.

A key estimate in the first step of the argument is provided by the following proposition:

Proposition 3.7 Assume that $b$ is a smooth function with compact support. Then for any $p \in[1, \infty)$ and $t \in \mathbb{R}$, we have the following estimate for the solution of the linearized equation (3.12):

$$
\sup _{x \in \mathbb{R}^{d}} E\left[\left|\frac{\partial}{\partial x} X_{t}^{x}\right|^{p}\right] \leq C_{d, p}\left(\|b\|_{\infty}\right)
$$

where $C_{d, p}$ is an increasing continuous function depending only on $d$ and $p$.

The proof of Proposition 3.7 relies on the following sequence of lemmas which provide estimates on expressions depending on the Gaussian distribution and its derivatives. To this end we define $P(t, z):=(2 \pi t)^{d / 2} e^{-|z|^{2} / 2 t}, t>0$, where $|z|$ is the Euclidean norm of a vector $z \in \mathbb{R}^{d}$.

Lemma 3.8 Let $\phi, h:[0,1] \times \mathbb{R}^{d} \rightarrow \mathbb{R}$ be measurable functions such that $|\phi(s, z)| \leq e^{-|z|^{2} / 3 s}$ and $\|h\|_{\infty} \leq 1$. Also let $\alpha, \beta \in\{0,1\}^{d}$ be multiindices such that $|\alpha|=|\beta|=1$. Then there exists a universal constant $C$ (independent of $\phi, h, \alpha$ and $\beta$ ) such that

$$
\left|\int_{1 / 2}^{1} \int_{t / 2}^{t} \int_{\mathbb{R}^{d}} \int_{\mathbb{R}^{d}} \phi(s, z) h(t, y) D^{\alpha} D^{\beta} P(t-s, y-z) d y d z d s d t\right| \leq C .
$$

Furthermore, there is a universal positive constant (also denoted by) $C$ such that for measurable functions $g$ and $h$ bounded by 1 , we have

$$
\left|\int_{1 / 2}^{1} \int_{t / 2}^{t} \int_{\mathbb{R}^{d}} \int_{\mathbb{R}^{d}} g(s, z) P(s, z) h(t, y) D^{\alpha} D^{\beta} P(t-s, y-z) d y d z d s d t\right| \leq C
$$


and

$$
\left|\int_{1 / 2}^{1} \int_{t / 2}^{t} \int_{\mathbb{R}^{d}} \int_{\mathbb{R}^{d}} g(s, z) D^{\gamma} P(s, z) h(t, y) D^{\alpha} D^{\beta} P(t-s, y-z) d y d z d s d t\right| \leq C .
$$

\section{Proof.}

We will only give a proof of the first estimate in the lemma. The proofs of the second and third estimates are left to the reader.

Denote the first integral in the lemma by $I$. Let $l, m \in \mathbb{Z}^{d}$ and define $[l, l+1):=$ $\left[l^{(1)}, l^{(1)}+1\right) \times \cdots \times\left[l^{(d)}, l^{(d)}+1\right)$ and similarly for $[m, m+1)$. Truncate the functions $\phi, h$ by setting $\phi_{l}(s, z):=\phi(s, z) 1_{[l, l+1)}(z)$ and $h_{m}(t, y):=h(t, y) 1_{[m, m+1)}(y)$.

In the first integral, we replace $\phi, h$ by $\phi_{l}, h_{m}$ respectively, and thus define

$$
I_{l, m}:=\int_{1 / 2}^{1} \int_{t / 2}^{t} \int_{\mathbb{R}^{d}} \int_{\mathbb{R}^{d}} \phi_{l}(s, z) h_{m}(t, y) D^{\alpha} D^{\beta} P(t-s, y-z) d y d z d s d t
$$

Therefore we can write $I=\sum_{l, m \in \mathbb{Z}^{d}} I_{l, m}$. Below we let $C$ be a generic constant that may vary from line to line.

Assume $\|l-m\|_{\infty}:=\max _{i}\left|l^{(i)}-m^{(i)}\right| \geq 2$. For $z \in[l, l+1)$ and $y \in[m, m+1)$ we have $|z-y| \geq\|l-m\|_{\infty}-1$. If $\alpha \neq \beta$ we have that

$$
D^{\alpha} D^{\beta} P(t-s, z-y)=\frac{\left(z^{(i)}-y^{(i)}\right)\left(z^{(j)}-y^{(j)}\right)}{(t-s)^{2}} P(t-s, y-z)
$$

for a suitable choice of $i, j$. Then we can find $C$ such that

$$
\left|D^{\alpha} D^{\beta} P(t-s, z-y)\right| \leq C e^{-\left(\|l-m\|_{\infty}-2\right)^{2} / 4} .
$$

If $\alpha=\beta$, we have

$$
\left(D^{\alpha}\right)^{2} P(t-s, y-z)=\left(\frac{\left(y^{(i)}-z^{(i)}\right)^{2}}{t-s}-1\right) \frac{P(t-s, y-z)}{t-s}
$$

and similarly we find $C$ such that

$$
\left|\left(D^{\alpha}\right)^{2} P(t-s, y-z)\right| \leq C e^{-\left(\|l-m\|_{\infty}-2\right)^{2} / 4} .
$$

In both cases we have $\left|I_{l, m}\right| \leq C e^{-|l|^{2} / 8} e^{-\left(\|l-m\|_{\infty}-2\right)^{2} / 4}$ and it follows that

$$
\sum_{\|l-m\|_{\infty} \geq 2}\left|I_{l, m}\right| \leq C
$$

Assume $\|l-m\|_{\infty} \leq 1$ and let $\hat{\phi}_{l}(s, u)$ and $\hat{h}_{m}(t, u)$ be the Fourier transform in the second variable, defined by

$$
\hat{h}_{m}(t, u):=(2 \pi)^{-d / 2} \int_{\mathbb{R}^{d}} h(t, x) e^{-i(u, x)} d x
$$

and similarly for $\hat{\phi}_{l}(s, u)$. By the Plancherel theorem we have that

$$
\int_{\mathbb{R}^{d}} \hat{\phi}_{l}(s, u)^{2} d u=\int_{\mathbb{R}^{d}} \phi_{l}(s, z)^{2} d z \leq C e^{-|l|^{2} / 6}
$$


for all $s \in[0,1]$ and

$$
\int_{\mathbb{R}^{d}} \hat{h}_{m}(t, u)^{2} d u=\int_{\mathbb{R}^{d}} h_{m}(t, y)^{2} d y \leq 1 .
$$

We can write

$$
I_{l, m}=\int_{1 / 2}^{1} \int_{t / 2}^{t} \int_{\mathbb{R}^{d}} \hat{\phi}_{l}(s, u) \hat{h}_{m}(t,-u) u^{(i)} u^{(j)}(t-s) e^{-(t-s)|u|^{2} / 2} d u d s d t .
$$

To see this, start with the right hand side. Then we have by Fubini's theorem

$$
\begin{aligned}
& \int_{\mathbb{R}^{d}} \hat{h}_{m}(t,-u) \hat{\phi}_{l}(s, u) u^{i} u^{j}(t-s) e^{-(t-s)|u|^{2} / 2} d u \\
& =(2 \pi)^{-d} \int_{\mathbb{R}^{d}} \int_{\mathbb{R}^{d}} \int_{\mathbb{R}^{d}} h_{m}(t, x) e^{i(u, x)} \phi_{l}(s, y) e^{-i(u, y)} u^{i} u^{j}(t-s) e^{-(t-s)|u|^{2} / 2} d u d x d y \\
& =\int_{\mathbb{R}^{d}} \int_{\mathbb{R}^{d}} h_{m}(t, x) \phi_{l}(s, y)(t-s)\left[(2 \pi)^{-d} \int_{\mathbb{R}^{d}} e^{i(u, x-y)} u^{i} u^{j} e^{-(t-s)|u|^{2} / 2} d u\right] d x d y .
\end{aligned}
$$

Now look at the expression in the square brackets. Substitute $v=\sqrt{t-s} u$ to get

$$
\begin{aligned}
& (2 \pi)^{-d} \int_{\mathbb{R}^{d}} e^{i(u, x-y)} u^{i} u^{j} e^{-(t-s)|u|^{2} / 2} d u \\
& =(2 \pi)^{-d}(t-s)^{-d / 2} \int_{\mathbb{R}^{d}} e^{i\left(\frac{v}{\sqrt{t-s}}, x-y\right)} \frac{v^{i}}{\sqrt{t-s}} \frac{v^{j}}{\sqrt{t-s}} e^{-|v|^{2} / 2} d v \\
& =(2 \pi)^{-d}(t-s)^{-d / 2}(t-s)^{-1} \int_{\mathbb{R}^{d}} e^{i\left(v, \frac{x-y}{\sqrt{t-s}}\right)} v^{i} v^{j} e^{-|v|^{2} / 2} d v .
\end{aligned}
$$

Now put $f(v)=e^{-|v|^{2} / 2}$ and $p(v)=v^{(i)} v^{(j)}$. From properties of the Fourier transform we know that $\widehat{p f}=D^{\alpha} D^{\beta} \hat{f}$ and $\hat{f}=f$. This gives that the above expression is equal to

$$
(2 \pi)^{-d / 2}(t-s)^{-d / 2}(t-s)^{-1} D^{\alpha} D^{\beta} f\left(\frac{x-y}{\sqrt{t-s}}\right)=(t-s)^{-1} D^{\alpha} D^{\beta} P(t-s, x-y) .
$$

This gives the equation (3.13).

Applying the inequality $a b \leq \frac{1}{2} a^{2} c+\frac{1}{2} b^{2} c^{-1}$ to (3.13) with $a=\hat{\phi}_{l}(s, u) u^{(i)}, b=\hat{h}_{m}(t,-u) u^{(j)}$ and $c=e^{|l|^{2} / 12}$ we get

$$
\begin{aligned}
\left|I_{l, m}\right| & \leq \frac{1}{2} \int_{1 / 2}^{1} \int_{t / 2}^{t} \int_{\mathbb{R}^{d}} \hat{\phi}_{l}(s, u)^{2}\left(u^{(i)}\right)^{2} e^{|l|^{2} / 12} e^{-(t-s)|u|^{2} / 2} d u d s d t \\
& +\frac{1}{2} \int_{1 / 2}^{1} \int_{t / 2}^{t} \int_{\mathbb{R}^{d}} \hat{h}_{m}(t,-u)^{2}\left(u^{(j)}\right)^{2} e^{-|l|^{2} / 12} e^{-(t-s)|u|^{2} / 2} d u d s d t \\
& \leq \frac{1}{2} \int_{1 / 2}^{1} \int_{t / 2}^{t} \int_{\mathbb{R}^{d}} \hat{\phi}_{l}(s, u)^{2}|u|^{2} e^{|l|^{2} / 12} e^{-(t-s)|u|^{2} / 2} d u d s d t \\
& +\frac{1}{2} \int_{1 / 2}^{1} \int_{t / 2}^{t} \int_{\mathbb{R}^{d}} \hat{h}_{m}(t,-u)^{2}|u|^{2} e^{-|l|^{2} / 12} e^{-(t-s)|u|^{2} / 2} d u d s d t .
\end{aligned}
$$


For the first term, integrate first with respect to $t$ in order to get

$$
\int_{1 / 2}^{1} \int_{t / 2}^{t} \int_{\mathbb{R}^{d}} \hat{\phi}_{l}(s, u)^{2}|u|^{2} e^{|l|^{2} / 12} e^{-(t-s)|u|^{2} / 2} d u d s d t \leq C e^{-|l|^{2} / 12}
$$

and for the second term, integrate with respect to $s$ first to get

$$
\int_{1 / 2}^{1} \int_{t / 2}^{t} \int_{\mathbb{R}^{d}} \hat{h}_{m}(t,-u)^{2}|u|^{2} e^{-|l|^{2} / 12} e^{-(t-s)|u|^{2} / 2} d u d s d t \leq C e^{-|l|^{2} / 12}
$$

which gives $\left|I_{l, m}\right| \leq C e^{-|l|^{2} / 12}$ and hence

$$
\sum_{\|l-m\|_{\infty} \leq 1}\left|I_{l, m}\right| \leq C
$$

Using the previous lemma we can show the following:

Lemma 3.9 Let $g, h:[0,1] \times \mathbb{R}^{d} \rightarrow \mathbb{R}$ be Borel-measurable and bounded by 1 and let $r \geq 0$. As before we let $\alpha, \beta, \gamma$ be multiindexes with length 1 . There exists a universal constant $C$ such that

$$
\begin{array}{r}
\left|\int_{t_{0}}^{t} \int_{t_{0}}^{t_{1}} \int_{\mathbb{R}^{d}} \int_{\mathbb{R}^{d}} g\left(t_{2}, z\right) P\left(t_{2}-t_{0}, z\right) h\left(t_{1}, y\right) D^{\alpha} D^{\beta} P\left(t_{1}-t_{2}, y-z\right)\left(t-t_{1}\right)^{r} d y d z d t_{2} d t_{1}\right| \\
\leq C(1+r)^{-1}\left(t-t_{0}\right)^{r+1}
\end{array}
$$

and

$$
\begin{array}{r}
\left|\int_{t_{0}}^{t} \int_{t_{0}}^{t_{1}} \int_{\mathbb{R}^{d}} \int_{\mathbb{R}^{d}} g\left(t_{2}, z\right) D^{\gamma} P\left(t_{2}-t_{0}, z\right) h\left(t_{1}, y\right) D^{\alpha} D^{\beta} P\left(t_{1}-t_{2}, y-z\right)\left(t-t_{1}\right)^{r} d y d z d t_{2} d t_{1}\right| \\
\leq C(1+r)^{-1 / 2}\left(t-t_{0}\right)^{r+1 / 2} .
\end{array}
$$

\section{Proof.}

We begin by proving the first estimate in the lemma for $t=1, t_{0}=0$. The following estimate holds for each integer $k \geq 0$ :

$$
\begin{array}{r}
\left|\int_{2^{-k-1}}^{2^{-k}} \int_{t / 2}^{t} \int_{\mathbb{R}^{d}} \int_{\mathbb{R}^{d}} g(s, z) P(s, z) h(t, y) D^{\alpha} D^{\beta} P(t-s, y-z)(1-t)^{r} d y d z d s d t\right| \\
\leq C\left(1-2^{-k-1}\right)^{r} 2^{-k} .
\end{array}
$$

To see this, use the fact $P(a t, z)=a^{-d / 2} P\left(t, a^{-1 / 2} z\right)$ and make the following substitutions in the second estimate in Lemma 3.8: $t^{\prime}:=2^{k} t$ and $s^{\prime}:=2^{k} s, z^{\prime}:=2^{k / 2} z$ and $y^{\prime}:=2^{k / 2} y$, $\tilde{h}(t, y):=\frac{(1-t)^{r}}{\left(1-2^{-k-1}\right)^{r}} h(t, y)$.

Summing the above inequalities over $k$ gives

$$
\left|\int_{0}^{1} \int_{t / 2}^{t} \int_{\mathbb{R}^{d}} \int_{\mathbb{R}^{d}} g(s, z) P(s, z) h(t, y) D^{\alpha} D^{\beta} P(t-s, y-z)(1-t)^{r} d y d z d s d t\right| \leq C(1+r)^{-1} .
$$


Moreover, it is easy to see that

$$
\begin{array}{r}
\left|\int_{0}^{1} \int_{0}^{t / 2} \int_{\mathbb{R}^{d}} \int_{\mathbb{R}^{d}} g(s, z) P(s, z) h(t, y) D^{\alpha} D^{\beta} P(t-s, y-z)(1-t)^{r} d y d z d s d t\right| \\
\leq C \int_{0}^{1} \int_{0}^{t / 2}(t-s)^{-1}(1-t)^{r} d s d t \leq C(1+r)^{-1},
\end{array}
$$

and combining these bounds gives the first assertion of the lemma for $t=1, t_{0}=0$. For general $t$ and $t_{0}$ use the change of variables $t_{1}^{\prime}:=\frac{t_{1}-t_{0}}{t-t_{0}}, t_{2}^{\prime}:=\frac{t_{2}-t_{0}}{t-t_{0}}, y^{\prime}:=\left(t-t_{0}\right)^{-1 / 2} y$ and $z^{\prime}:=\left(t-t_{0}\right)^{-1 / 2} z$.

The second assertion of the lemma is proved similarly.

We now turn to the following key estimate (cf. [6, Proposition 2.2]):

Lemma 3.10 Let $B$ be a d-dimensional Brownian Motion starting from the origin and $b_{1}, \ldots, b_{n}$ be compactly supported continuously differentiable functions $b_{i}:[0,1] \times \mathbb{R}^{d} \rightarrow \mathbb{R}$ for $i=1,2, \ldots n$. Let $\alpha_{i} \in\{0,1\}^{d}$ be a multiindex such that $\left|\alpha_{i}\right|=1$ for $i=1,2, \ldots, n$. Then there exists a universal constant $C$ (independent of $\left\{b_{i}\right\}_{i}, n$, and $\left\{\alpha_{i}\right\}_{i}$ ) such that

$$
\begin{gathered}
\left|E\left[\int_{t_{0}<t_{1}<\cdots<t_{n}<t}\left(\prod_{i=1}^{n} D^{\alpha_{i}} b_{i}\left(t_{i}, x+B_{t_{i}}\right)\right) d t_{1} \ldots d t_{n}\right]\right| \\
\leq \frac{C^{n} \prod_{i=1}^{n}\left\|b_{i}\right\|_{\infty}\left(t-t_{0}\right)^{n / 2}}{\Gamma\left(\frac{n}{2}+1\right)}
\end{gathered}
$$

where $\Gamma$ is the Gamma-function and $x \in \mathbb{R}^{d}$. Here $D^{\alpha_{i}}$ denotes the partial derivative with respect to the $j^{\prime}$ th space variable, where $j$ is the position of the 1 in $\alpha_{i}$.

Proof. Without loss of generality, assume that $\left\|b_{i}\right\|_{\infty} \leq 1$ for $i=1,2 \ldots, n$. Using the Gaussian density we write the left hand side of the estimate (3.14) in the form

$$
\left|\int_{t_{0}<t_{1}<\cdots<t_{n}<t} \int_{\mathbb{R}^{d n}} \prod_{i=1}^{n} D^{\alpha_{i}} b_{i}\left(t_{i}, x+z_{i}\right) P\left(t_{i}-t_{i-1}, z_{i}-z_{i-1}\right) d z_{1} \ldots d z_{n} d t_{1} \ldots d t_{n}\right| .
$$

Introduce the notation

$J_{n}^{\alpha}\left(t_{0}, t, z_{0}\right)=\int_{t_{0}<t_{1}<\cdots<t_{n}<t} \int_{\mathbb{R}^{d n}} \prod_{i=1}^{n} D^{\alpha_{i}} b_{i}\left(t_{i}, x+z_{i}\right) P\left(t_{i}-t_{i-1}, z_{i}-z_{i-1}\right) d z_{1} \ldots d z_{n} d t_{1} \ldots d t_{n}$

where $\alpha=\left(\alpha_{1}, \ldots \alpha_{n}\right) \in\{0,1\}^{n d}$. We shall show that $\left|J_{n}^{\alpha}\left(t_{0}, t, z_{0}\right)\right| \leq C^{n}\left(t-t_{0}\right)^{n / 2} / \Gamma(n / 2+1)$, thus proving the proposition.

To do this, we will use integration by parts to shift the derivatives from the $b_{i}$ 's onto the Gaussian kernel. This will be done by introducing the alphabet

$$
\mathcal{A}(\alpha)=\left\{P, D^{\alpha_{1}} P, \ldots, D^{\alpha_{n}} P, D^{\alpha_{1}} D^{\alpha_{2}} P, \ldots D^{\alpha_{n-1}} D^{\alpha_{n}} P\right\}
$$

where $D^{\alpha_{i}}, D^{\alpha_{i}} D^{\alpha_{i+1}}$ denotes the derivatives in $z$ of $P(t, z)$. 
Take a string $S=S_{1} \cdots S_{n}$ in $\mathcal{A}(\alpha)$ and define

$$
I_{S}^{\alpha}\left(t_{0}, t, z_{0}\right)=\int_{t_{0}<\cdots<t_{n}<t} \int_{\mathbb{R}^{d n}} \prod_{i=1}^{n} b_{i}\left(t_{i}, x+z_{i}\right) S_{i}\left(t_{i}-t_{i-1}, z_{i}-z_{i-1}\right) d z_{1} \ldots d z_{n} d t_{1} \ldots d t_{n} .
$$

We will need only a special type of strings: Say that a string is allowed if, when all the $D^{\alpha_{i}} P$ 's are removed from the string, a string of the form $P \cdot D^{\alpha_{s}} D^{\alpha_{s+1}} P \cdot P \cdot D^{\alpha_{s+1}} D^{\alpha_{s+2}} P \cdots P$. $D^{\alpha_{r}} D^{\alpha_{r+1}} P$ for $s \geq 1, r \leq n-1$ remains. Also, we will require that the first derivatives $D^{\alpha_{i}} P$ are written in an increasing order with respect to $i$.

We now claim that

$$
J_{n}^{\alpha}\left(t_{0}, t, z_{0}\right)=\sum_{j=1}^{2^{n-1}} \epsilon_{j} I_{S^{j}}^{\alpha}\left(t_{0}, t, z_{0}\right)
$$

where each $\epsilon_{j}$ is either -1 or 1 and each $S^{j}$ is an allowed string in $\mathcal{A}(\alpha)$. To see this, we proceed by induction on $n \geq 1$ :

The claim obviously holds for $n=1$. Assume that it holds for $n \geq 1$, and let $b_{0}$ be another function satisfying the requirements of the lemma. Likewise with $\alpha_{0}$. Then

$$
\begin{aligned}
J_{n+1}^{\left(\alpha_{0}, \alpha\right)}\left(t_{0}, t, z_{0}\right)= & \int_{t_{0}}^{t} \int_{\mathbb{R}^{d}} D^{\alpha_{0}} b_{0}\left(t_{1}, x+z_{1}\right) P\left(t_{1}-t_{0}, z_{1}-z_{0}\right) J_{n}^{\alpha}\left(t_{1}, t, z_{1}\right) d z_{1} d t_{1} \\
= & -\int_{t_{0}}^{t} \int_{\mathbb{R}^{d}} b_{0}\left(t_{1}, x+z_{1}\right) D^{\alpha_{0}} P\left(t_{1}-t_{0}, z_{1}-z_{0}\right) J_{n}^{\alpha}\left(t_{1}, t, z_{1}\right) d z_{1} d t_{1} \\
& -\int_{t_{0}}^{t} \int_{\mathbb{R}^{d}} b_{0}\left(t_{1}, x+z_{1}\right) P\left(t_{1}-t_{0}, z_{1}-z_{0}\right) D^{\alpha_{0}} J_{n}^{\alpha}\left(t_{1}, t, z_{1}\right) d z_{1} d t_{1} .
\end{aligned}
$$

Notice that

$$
D^{\alpha_{0}} I_{S}^{\alpha}\left(t_{1}, t, z_{1}\right)=-I_{\tilde{S}}^{\left(\alpha_{0}, \alpha\right)}\left(t_{1}, t, z_{1}\right)
$$

where

$$
\tilde{S}= \begin{cases}D^{\alpha_{0}} P \cdot S_{2} \cdots S_{n} & \text { if } S=P \cdot S_{2} \cdots S_{n} \\ D^{\alpha_{0}} D^{\alpha_{1}} P \cdot S_{2} \cdots S_{n} & \text { if } S=D^{\alpha_{1}} P \cdot S_{2} \cdots S_{n}\end{cases}
$$

Here, $\tilde{S}$ is not an allowed string in $\mathcal{A}(\alpha)$. So from the induction hypothesis $D^{\alpha_{0}} J_{n}^{\alpha}\left(t_{0}, t, z_{0}\right)=$ $\sum_{j=1}^{2^{n-1}}-\epsilon_{j} I_{\tilde{S}}^{\left(\alpha_{0}, \alpha\right)}\left(t_{0}, t, z_{0}\right)$ this gives

$$
J_{n+1}^{\left(\alpha_{0}, \alpha\right)}=\sum_{j=1}^{2^{n-1}}-\epsilon_{j} I_{D^{\alpha} 0 P \cdot S^{j}}^{\left(\alpha_{0}, \alpha\right)}+\sum_{j=1}^{2^{n-1}} \epsilon_{j} I_{P \cdot \tilde{S}^{j}} .
$$

It is easily checked that when $S^{j}$ is an allowed string in $\mathcal{A}(\alpha)$, both $D^{\alpha_{0}} P \cdot S^{j}$ and $P \cdot \tilde{S}^{j}$ are allowed strings in $\mathcal{A}\left(\alpha_{0}, \alpha\right)$.

This proves the claim.

For the rest of the proof of Lemma 3.10 we will bound $I_{S}^{\alpha}$ when $S$ is an allowed string, i.e. we will show that there is a positive constant $M$ such that

$$
I_{S}^{\alpha}\left(t_{0}, t, z_{0}\right) \leq \frac{M^{n}\left(t-t_{0}\right)^{n / 2}}{\Gamma\left(\frac{n}{2}+1\right)} .
$$

for all integers $n \geq 1$ and for each allowed string $S$ in the alphabet $\mathcal{A}(\alpha)$.

We proceed by induction on $n \geq 0$ : The case $n=0$ is immediate, so assume $n>0$ and that this holds for all allowed strings of length less than $n$. We divide into three cases: 
1. $S=D^{\alpha_{1}} P \cdot S^{\prime}$ where $S^{\prime}$ is a string in $\mathcal{A}\left(\alpha^{\prime}\right)$ and $\alpha^{\prime}:=\left(\alpha_{2}, \ldots, \alpha_{n}\right)$

2. $S=P \cdot D^{\alpha_{1}} D^{\alpha_{2}} P \cdot S^{\prime}$ where $S^{\prime}$ is a string in $\mathcal{A}\left(\alpha^{\prime}\right)$ and $\alpha^{\prime}:=\left(\alpha_{3}, \ldots, \alpha_{n}\right)$

3. $S=P \cdot D^{\alpha_{1}} P \cdots D^{\alpha_{m}} P \cdot D^{\alpha_{m+1}} D^{\alpha_{m+2}} P \cdot S^{\prime}$ where $S^{\prime}$ is a string in $\mathcal{A}\left(\alpha^{\prime}\right)$ and $\alpha^{\prime}:=$ $\left(\alpha_{m+3}, \ldots, \alpha_{n}\right)$.

In all the above cases, $S^{\prime}$ is an allowed string in the alphabet.

1. We use the inductive hypothesis to bound $I_{S^{\prime}}^{\alpha^{\prime}}\left(t_{1}, t, z_{1}\right)$ and the bound

$$
\int_{\mathbb{R}^{d}}\left|D^{\alpha} P(t, z)\right| d z \leq C t^{-1 / 2}
$$

to get

$$
\begin{aligned}
\left|I_{S}^{\alpha}\left(t_{0}, t, z_{0}\right)\right| & =\left|\int_{t_{0}}^{t} \int_{\mathbb{R}^{d}} b_{1}\left(t_{1}, z_{1}\right) D^{\alpha_{1}} P\left(t_{1}-t_{0}, z_{1}-z_{0}\right) I_{S^{\prime}}^{\alpha^{\prime}}\left(t_{1}, t, z_{1}\right) d z_{1} d t_{1}\right| \\
& \leq \frac{M^{n-1}}{\Gamma\left(\frac{n+1}{2}\right)} \int_{t_{0}}^{t}\left(t-t_{1}\right)^{(n-1) / 2} \int_{\mathbb{R}^{d}}\left|D^{\alpha_{1}} P\left(t_{1}-t_{0}, z_{1}-z_{0}\right)\right| d z_{1} d t_{1} \\
& \leq \frac{M^{n-1} C}{\Gamma\left(\frac{n+1}{2}\right)} \int_{t_{0}}^{t}\left(t-t_{1}\right)^{(n-1) / 2}\left(t_{1}-t_{0}\right)^{-1 / 2} d t_{1} \\
& =\frac{M^{n-1} C \sqrt{\pi}\left(t-t_{0}\right)^{k / 2}}{\Gamma\left(\frac{n}{2}+1\right)} .
\end{aligned}
$$

The result follows if $M \geq \max \{C \sqrt{\pi}, 1\}$.

2. For this case we can write

$$
\begin{aligned}
& I_{S}^{\alpha}\left(t_{0}, t, z_{0}\right)=\int_{t_{0}}^{t} \int_{t_{1}}^{t} \int_{\mathbb{R}^{d}} \int_{\mathbb{R}^{d}} b_{1}\left(t_{1}, z_{1}\right) b_{2}\left(t_{2}, z_{2}\right) \\
& \quad \times P\left(t_{1}-t_{0}, z_{1}-z_{0}\right) D^{\alpha_{1}} D^{\alpha_{2}} P\left(t_{2}-t_{1}, z_{2}-z_{1}\right) I_{S^{\prime}}^{\alpha^{\prime}}\left(t_{2}, t, z_{2}\right) d z_{1} d z_{2} d t_{2} d t_{1} .
\end{aligned}
$$

We set $h\left(t_{2}, z_{2}\right):=b_{2}\left(t_{2}, z_{2}\right) I_{S^{\prime}}^{\alpha^{\prime}}\left(t_{2}, z_{2}\right)\left(t-t_{2}\right)^{1-n / 2}$ so that by the inductive hypothesis we have

$$
\|h\|_{\infty} \leq M^{n-2} / \Gamma(n / 2) .
$$

Use the above estimate in the first assertion of Lemma 3.9 with $g=b_{1}$ and integrate with respect to $t_{2}$ first, to get

$$
\left|I_{S}^{\alpha}\left(t_{0}, t, z_{0}\right)\right| \leq \frac{C M^{n-2}\left(t-t_{0}\right)^{n / 2}}{n \Gamma(n / 2)},
$$

and the result follows if $M \geq \max \{C, 1\}$.

3. We have

$$
\begin{aligned}
I_{S}^{\alpha}\left(t_{0}, t, z_{0}\right) & =\int_{t_{0}<\ldots t_{m+2}<t} \int_{\mathbb{R}^{(m+2) d}} P\left(t_{1}-t_{0}, z_{1}-z_{0}\right) \prod_{j=1}^{m+2} b_{j}\left(t_{j}, z_{j}\right) \\
& \times \prod_{j=2}^{m} D^{\alpha_{j}} P\left(t_{j}-t_{j-1}, z_{j}-z_{j-1}\right) D^{\alpha_{m+1}} D^{\alpha_{m+2}} P\left(t_{m+2}-t_{m+1}, z_{m+2}-z_{m+1}\right) \\
& \times I_{S^{\prime}}^{\alpha^{\prime}}\left(t_{m+2}, t, z_{m+2}\right) d z_{1} \ldots d z_{m+2} d t_{1} \ldots d t_{m+2} .
\end{aligned}
$$


Set $h\left(t_{m+2}, z_{m+2}\right):=b_{m+2}\left(t_{m+2}, z_{m+2}\right) I_{S^{\prime}}^{\alpha^{\prime}}\left(t_{m+2}, t, z\right)\left(t-t_{m+2}\right)^{(2+m-n) / 2}$. Then from the inductive hypothesis we have $\|h\|_{\infty} \leq M^{n-m-2} / \Gamma((n-m) / 2)$. Define

$$
\begin{aligned}
A\left(t_{m}, z_{m}\right) & :=\int_{t_{m}}^{t} \int_{t_{m+1}}^{t} \int_{\mathbb{R}^{2 d}} b_{m+1}\left(t_{m+1}, z_{m+1}\right) h\left(t_{m+2}, z_{m+2}\right) \\
& \times\left(t-t_{m+2}\right)^{(n-m-2) / 2} D^{\alpha_{m}} P\left(t_{m+1}-t_{m}, z_{m+1}-z\right) \\
& \times D^{\alpha_{m+1}} D^{\alpha_{m+2}} P\left(t_{m+2}-t_{m+1}, z_{m+2}-z_{m+1}\right) d z_{m+1} d z_{m+2} d t_{m+1} d t_{m+2} .
\end{aligned}
$$

Then Lemma 3.9 implies that

$$
\left|A\left(t_{m}, z_{m}\right)\right| \leq \frac{C(n-m)^{-1 / 2} M^{n-m-2}\left(t-t_{m}\right)^{(n-m-1) / 2}}{\Gamma\left(\frac{n-m}{2}\right)} .
$$

Using this in

$$
\begin{aligned}
I_{S}^{\alpha}\left(t_{0}, t, z_{0}\right)= & \int_{t_{0}<\ldots t_{m+2}<t} \int_{\mathbb{R}^{(m+2) d}} P\left(t_{1}-t_{0}, z_{1}-z_{0}\right) \prod_{j=1}^{m} b_{j}\left(t_{j}, z_{j}\right) \\
& \times \prod_{j=1}^{m-1} D^{\alpha_{j}} P\left(t_{j}-t_{j-1}, z_{j}-z_{j-1}\right) \Omega\left(t_{m}, z_{m}\right) d z_{1} \ldots d z_{m} d t_{1} \ldots d t_{m},
\end{aligned}
$$

and using the bound (3.15) several times gives

$$
\begin{aligned}
\left|I_{S}^{\alpha}\left(t_{0}, t, z_{0}\right)\right| & \leq C^{m+1}(n-m)^{-1 / 2} \frac{M^{n-m-2}}{\Gamma((n-m) / 2)} \\
& \times \int_{t_{0}<\ldots t_{m}<t}\left(t_{2}-t_{1}\right)^{-1 / 2} \ldots\left(t_{m}-t_{m-1}\right)^{-1 / 2}\left(t-t_{m}\right)^{(n-m-1) / 2} d t_{1} \ldots d t_{m} \\
& =C^{m+1}(n-m)^{-1 / 2} \frac{M^{n-m-2} \pi^{(m-1) / 2} \Gamma\left(\frac{n-m+1}{2}\right)}{\Gamma\left(\frac{n-m}{2}\right) \Gamma\left(\frac{n}{2}+1\right)}\left(t-t_{0}\right)^{n / 2} .
\end{aligned}
$$

We can choose $M$ so large that the result holds. This completes the induction argument.

We are now ready to complete the proof of Proposition 3.7.

Proof of Proposition 3.7. Let $t \in[0,1]$. Iterating the linearized equation (3.12) we obtain

$$
\frac{\partial}{\partial x} X_{t}^{x}=\mathcal{I}_{d}+\sum_{n=1}^{\infty} \int_{0<s_{1}<\ldots s_{n}<t} b^{\prime}\left(s_{1}, X_{s_{1}}^{x}\right): \cdots: b^{\prime}\left(s_{n}, X_{s_{n}}^{x}\right) d s_{1} \ldots d s_{n} .
$$

Let $p \in[1, \infty)$ and choose $r, s \in[1, \infty)$ such that $s p=2^{q}$ for some integer $q$ and $\frac{1}{r}+\frac{1}{s}=1$. Then by Girsanov's theorem and Hölder's inequality

$$
\begin{aligned}
& E\left[\left|\frac{\partial}{\partial x} X_{t}^{x}\right|^{p}\right]=E\left[\left|\mathcal{I}_{d}+\sum_{n=1}^{\infty} \int_{0<s_{1}<\ldots s_{n}<t} b^{\prime}\left(s_{1}, x+B_{s_{1}}\right): \cdots: b^{\prime}\left(s_{n}, x+B_{s_{n}}\right) d s_{1} \ldots d s_{n}\right|^{p}\right. \\
&\left.\times \mathcal{E}\left(\int_{0}^{1} b\left(u, x+B_{u}\right) d B_{u}\right)\right] \\
& \leq C_{1}\left(\|b\|_{\infty}\right)\left\|\mathcal{I}_{d}+\sum_{n=1}^{\infty} \int_{0<s_{1}<\ldots s_{n}<t} b^{\prime}\left(s_{1}, x+B_{s_{1}}\right): \cdots: b^{\prime}\left(s_{n}, x+B_{s_{n}}\right) d s_{1} \ldots d s_{n}\right\| \|_{L^{s p}\left(\mu, \mathbb{R}^{d \times d}\right)}^{p},
\end{aligned}
$$


where $\mathcal{E}\left(\int_{0}^{1} b\left(u, x+B_{u}\right) d B_{u}\right)$ is the Doleans-Dade exponential of the martingale

$$
\int_{0}^{1} b\left(u, x+B_{u}\right) d B_{u}=\sum_{j=1}^{d} \int_{0}^{1} b^{(j)}\left(u, x+B_{u}\right) d B_{u}^{j}
$$

and $C_{1}$ is a continuous increasing function.

Then we obtain

$$
\begin{gathered}
E\left|\frac{\partial}{\partial x} X_{t}^{x}\right|^{p} \\
\leq C_{1}\left(\|b\|_{\infty}\right)\left\|\mathcal{I}_{d}+\sum_{n=1}^{\infty} \int_{0<s_{1}<\ldots s_{n}<t} b^{\prime}\left(s_{1}, x+B_{s_{1}}\right): \cdots: b^{\prime}\left(s_{n}, x+B_{s_{n}}\right) d s_{1} \ldots d s_{n}\right\|_{L^{s p}\left(\mu, \mathbb{R}^{d \times d}\right)}^{p} \\
\leq C_{1}\left(\|b\|_{\infty}\right)\left(1+\sum_{n=1}^{\infty} \sum_{i, j=1}^{d} \sum_{l_{1}, \ldots l_{n-1}=1}^{d} \| \int_{t<s_{1}<\cdots<s_{n}<s} \frac{\partial}{\partial x_{l_{1}}} b^{(i)}\left(s_{1}, x+B_{s_{1}}\right) \frac{\partial}{\partial x_{l_{2}}} b^{\left(l_{1}\right)}\left(s_{2}, x+B_{s_{2}}\right) \ldots\right. \\
\left.\cdots \frac{\partial}{\partial x_{j}} b^{\left(l_{n-1}\right)}\left(s_{n}, x+B_{s_{n}}\right) d s_{1} \ldots d s_{n} \|_{L^{p s}(\mu ; \mathbb{R})}\right)^{p} .
\end{gathered}
$$

Now consider the expression

$$
A:=\int_{0<s_{1}<\cdots<s_{n}<t} \frac{\partial}{\partial x_{l_{1}}} b^{(i)}\left(s_{1}, x+B_{s_{1}}\right) \frac{\partial}{\partial x_{l_{2}}} b^{\left(l_{1}\right)}\left(s_{2}, x+B_{s_{2}}\right) \ldots \frac{\partial}{\partial x_{l_{n}}} b^{\left(l_{n}\right)}\left(s_{n}, x+B_{s_{n}}\right) d s_{1} \ldots d s_{n} .
$$

Then, using (deterministic) integration by parts, repeatedly, it is easy to see that $A^{2}$ can be written as a sum of at most $2^{2 n}$ terms of the form

$$
\int_{0<s_{1}<\cdots<s_{2 n}<t} g_{1}\left(s_{1}\right) \ldots g_{2 n}\left(s_{2 n}\right) d s_{1} \ldots d s_{2 n},
$$

where $g_{l} \in\left\{\frac{\partial}{\partial x_{j}} b^{(i)}(\cdot, x+B):. 1 \leq i, j \leq d\right\}, l=1,2 \ldots 2 n$. Similarly, by induction it follows that $A^{2^{q}}$ is the sum of at most $2^{q 2^{q} n}$ terms of the form

$$
\int_{0<s_{1}<\cdots<s_{2 n}<t} g_{1}\left(s_{1}\right) \ldots g_{2^{q} n}\left(s_{2^{q} n}\right) d s_{1} \ldots d s_{2^{q} n}
$$

Combining this with Lemma 3.10, we obtain the following estimate:

$$
\begin{gathered}
\left\|\int_{0<s_{1}<\cdots<s_{n}<t} \frac{\partial}{\partial x_{l_{1}}} b^{(i)}\left(s_{1}, x+B_{s_{1}}\right) \frac{\partial}{\partial x_{l_{2}}} b^{\left(l_{1}\right)}\left(s_{2}, x+B_{s_{2}}\right) \ldots \frac{\partial}{\partial x_{j}} b^{\left(l_{n-1}\right)}\left(s_{n}, x+B_{s_{n}}\right) d s_{1} \ldots d s_{n}\right\|_{L^{2^{q}}(\mu ; \mathbb{R})} \\
\leq\left(\frac{2^{q 2^{q} n} C^{2^{q} n}\|b\|_{\infty}^{2^{q}} t^{2^{q-1} n}}{\Gamma\left(2^{q-1} n+1\right)}\right)^{2^{-q}} \leq \frac{2^{q n} C^{n}\|b\|_{\infty}^{n}}{\left(\left(2^{q-1} n\right) !\right)^{2^{-q}}} .
\end{gathered}
$$

Then it follows that

$$
\begin{gathered}
E\left[\left\|\frac{\partial}{\partial x} X_{t}^{x}\right\|^{p}\right] \\
\leq C_{1}\left(\|b\|_{\infty}\right)\left(1+\sum_{n=1}^{\infty} \frac{d^{n+2} 2^{q n} C^{n}\|b\|_{\infty}^{n}}{\left(\left(2^{q-1} n\right) !\right)^{2-q}}\right)^{p}=C_{d, p}\left(\|b\|_{\infty}\right) .
\end{gathered}
$$

The right hand side of this inequality is independent of $x \in \mathbb{R}^{d}$, and the result follows. 
For the remainder of the paper we will fix a bounded and measurable $b:[0,1] \times \mathbb{R}^{d} \rightarrow \mathbb{R}^{d}$. It is proved in [39] and [26] that the corresponding SDE (3.7) has a unique strong solution, denoted $X^{s, x}$. Suppose now $b_{n}:[0,1] \times \mathbb{R}^{d} \rightarrow \mathbb{R}^{d}$ be a sequence of compactly supported smooth functions. Suppose that $b_{n}(t, x) \longrightarrow b(t, x) d t \times d x-$ a.e. and $\left|b_{n}(t, x)\right| \leq M<\infty$ for all $n, t, x$ for some constant $M$. Denote by $X^{n, s, x}$ the solution of (3.7) associated with the coefficient $b_{n}, n \geq 1$. We then have the following

Lemma 3.11 The sequence $X_{t}^{n, s, x}$ converges weakly in $L^{2}\left(\Omega ; \mathbb{R}^{d}\right)$ to $X_{t}^{s, x}$.

Remark 3.12 Assume $\psi \in L^{\infty}\left([0,1] \times \Omega ; \mathbb{R}^{d}\right)$ adapted to the filtration generated by the Brownian motion. Then we can bound the Doleans-Dade exponential $\mathcal{E}\left(\int_{0}^{1} \psi(u) d B_{u}\right)$ in $L^{p}(\Omega)$ by an increasing continuous function of $\|\psi\|_{L^{\infty}([0,1] \times \Omega)}$.

To see this, notice that $M_{t}:=\mathcal{E}\left(\int_{0}^{t} \psi(u) d B_{u}\right)$ is the unique solution to the linear $S D E$

$$
d M_{t}=M(t) \psi(t) d B_{t}, M_{0}=1 .
$$

By Itô's formula we get

$$
\begin{gathered}
E\left[M_{t}^{p}\right]=1+\frac{p(p-1)}{2} \int_{0}^{t} E\left[M_{u}^{p}|\psi(u)|^{2}\right] d u \\
\leq 1+\frac{p(p-1)}{2}\|\psi\|_{L^{\infty}([0,1] \times \Omega)} \int_{0}^{t} E\left[M_{u}^{p}\right] d u \leq \exp \left\{\frac{t p(p-1)\|\psi\|_{L^{\infty}([0,1] \times \Omega)}}{2}\right\}
\end{gathered}
$$

where we have used Grönwall's lemma in the last inequality.

Proof of Lemma 3.11. For simplicity, consider $d=1$ and $s=0$. We start by noting that the set

$$
\left\{\mathcal{E}\left(\int_{0}^{1} h(u) d B_{u}\right): h \in C_{b}^{1}(\mathbb{R})\right\}
$$

spans a dense subspace of $L^{2}(\Omega ; \mathbb{R})$. So it suffices to prove the convergence

$$
E\left[X_{t}^{n, x} \mathcal{E}\left(\int_{0}^{1} h(u) d B_{u}\right)\right] \rightarrow E\left[X_{t}^{x} \mathcal{E}\left(\int_{0}^{1} h(u) d B_{u}\right)\right] .
$$

By the Cameron-Martin theorem we have

$$
E\left[X_{t}^{x} \mathcal{E}\left(\int_{0}^{1} h(u) d B_{u}\right)\right]=\int_{\Omega} X_{t}^{x}(\omega+h) d \mu(\omega) .
$$

The function $(u, x) \mapsto b(u, x)+h^{\prime}(u)$ is still bounded, and so $X_{t}^{x}(\cdot+h)$ must coincide with the solution to (3.7) when $b$ is replaced by $b+h^{\prime}$. Hence by uniqueness in law of (3.7) we may write

$$
E\left[X_{t}^{x} \mathcal{E}\left(\int_{0}^{1} h(u) d B_{u}\right)\right]=E\left[\left(x+B_{t}\right) \mathcal{E}\left(\int_{0}^{1} b\left(u, x+B_{u}\right)+h^{\prime}(u) d B_{u}\right)\right],
$$

and similary for $X_{t}^{n, x}$. We thus get

$$
E\left[X_{t}^{n, x} \mathcal{E}\left(\int_{0}^{1} h(u) d B_{u}\right)\right]-E\left[X_{t}^{x} \mathcal{E}\left(\int_{0}^{1} h(u) d B_{u}\right)\right]
$$




$$
=E\left[\left(x+B_{t}\right)\left(\mathcal{E}\left(\int_{0}^{1} b_{n}\left(u, x+B_{u}\right)+h^{\prime}(u) d B_{u}\right)-\mathcal{E}\left(\int_{0}^{1} b\left(u, x+B_{u}\right)+h^{\prime}(u) d B_{u}\right)\right)\right] .
$$

Using the inequality $\left|e^{a}-e^{b}\right| \leq\left|e^{a}+e^{b}\right||a-b|$, Hölders inequality and Burkholder-DavisGundy inequality we find a constant $C$ such that the above is bounded by

$$
\begin{gathered}
C\left(E\left[\left(\mathcal{E}\left(\int_{0}^{1} b_{n}\left(u, x+B_{u}\right)+h^{\prime}(u) d B_{u}\right)+\mathcal{E}\left(\int_{0}^{1} b\left(u, x+B_{u}\right)+h^{\prime}(u) d B_{u}\right)\right)^{4}\right]\right)^{1 / 4} \times \\
\left(E \left[\left(\int_{0}^{1}\left(b_{n}\left(u, x+B_{u}\right)-b\left(u, x+B_{u}\right)\right)^{2} d u\right)^{2}\right.\right. \\
\left.\left.+\left(\int_{0}^{1}\left(b\left(u, x+B_{u}\right)+h^{\prime}(u)\right)^{2}-\left(b_{n}\left(u, x+B_{u}\right)+h^{\prime}(u)\right)^{2} d u\right)^{4}\right]\right)^{1 / 4} \cdot
\end{gathered}
$$

From Remark 3.12, since $b_{n}$ is uniformly bounded we get that $\left\{\mathcal{E}\left(\int_{0}^{1} b_{n}\left(u, x+B_{u}\right)+h^{\prime}(u) d B_{u}\right)\right\}_{n}$ is bounded in $L^{4}(\Omega)$, so that the first factor above is uniformly bounded. The latter factor converges to zero by bounded convergence.

In [26] the authors show that the sequence $X_{t}^{n, s, x}$ is relatively compact in $L^{2}\left(\Omega ; \mathbb{R}^{d}\right.$ ) (in the strong topology). Thus $\left\{X_{t}^{n, x}\right\}$ has a converging subsequence, which, by Lemma 3.11 has to converge to $X_{t}^{x}$. Since every subsequence of $\left\{X_{t}^{n, x}\right\}$ has a further subsequence converging to the same limit $X_{t}^{x}$, the full sequence is convergent in $L^{2}\left(\Omega ; \mathbb{R}^{d}\right)$ (with strong topology).

As a consequence of Proposition 3.7 and the above discussion we obtain the following result:

Corollary 3.13 Let $X^{s, x}$ be the unique strong solution to the $S D E$ (3.7) and $q>1$ an integer. Then there exists a constant $C=C\left(d,\|b\|_{\infty}, q\right)<\infty$ such that

$$
E\left[\left|X_{t_{1}}^{s_{1}, x_{1}}-X_{t_{2}}^{s_{2}, x_{2}}\right|^{q}\right] \leq C\left(\left|s_{1}-s_{2}\right|^{q / 2}+\left|t_{1}-t_{2}\right|^{q / 2}+\left|x_{1}-x_{2}\right|^{q}\right)
$$

for all $s_{1}, s_{2}, t_{1}, t_{2}, x_{1}, x_{2}$.

In particular, there exists a continuous version of the random field $(s, t, x) \longmapsto X_{t}^{s, x}$ with Hölder continuous trajectories of Hölder constant $\alpha<\frac{1}{2}$ in $s, t$ and $\alpha<1$ in $x$, locally (see [22]).

Proof. Retain the above notation. Without loss of generality let $0 \leq s_{1}<s_{2}<t_{1}<t_{2}$. 
Then

$$
\begin{aligned}
& X_{t_{1}}^{n, s_{1}, x_{1}}-X_{t_{2}}^{n, s_{2}, x_{2}} \\
= & x_{1}-x_{2}+\int_{s_{1}}^{t_{1}} b_{n}\left(u, X_{u}^{n, s_{1}, x_{1}}\right) d u-\int_{s_{2}}^{t_{2}} b_{n}\left(u, X_{u}^{n, s_{2}, x_{2}}\right) d u \\
& +\left(B_{t_{1}}-B_{s_{1}}\right)-\left(B_{t_{2}}-B_{s_{2}}\right) \\
= & x_{1}-x_{2}+\int_{s_{1}}^{s_{2}} b_{n}\left(u, X_{u}^{n, s_{1}, x_{1}}\right) d u+\int_{s_{2}}^{t_{1}} b_{n}\left(u, X_{u}^{n, s_{1}, x_{1}}\right) d u \\
& -\int_{s_{2}}^{t_{1}} b_{n}\left(u, X_{u}^{n, s_{2}, x_{2}}\right) d u-\int_{t_{1}}^{t_{2}} b_{n}\left(u, X_{u}^{n, s_{2}, x_{2}}\right) d u \\
& +\left(B_{t_{1}}-B_{t_{2}}\right)+\left(B_{s_{2}}-B_{s_{1}}\right) \\
= & x_{1}-x_{2}+\int_{s_{1}}^{s_{2}} b_{n}\left(u, X_{u}^{n, s_{1}, x_{1}}\right) d u-\int_{t_{1}}^{t_{2}} b_{n}\left(u, X_{u}^{n, s_{2}, x_{2}}\right) d u \\
& +\int_{s_{2}}^{t_{1}}\left(b_{n}\left(u, X_{u}^{n, s_{1}, x_{1}}\right)-b_{n}\left(u, X_{u}^{n, s_{1}, x_{2}}\right)\right) d u \\
& +\int_{s_{2}}^{t_{1}}\left(b_{n}\left(u, X_{u}^{n, s_{1}, x_{2}}\right)-b_{n}\left(u, X_{u}^{n, s_{2}, x_{2}}\right)\right) d u \\
& +\left(B_{t_{1}}-B_{t_{2}}\right)+\left(B_{s_{2}}-B_{s_{1}}\right) .
\end{aligned}
$$

So due to the uniform boundedness of $b_{n}, n \geq 1$ we get

$$
\begin{aligned}
& E\left[\left|X_{t_{1}}^{n, s_{1}, x_{1}}-X_{t_{2}}^{n, s_{2}, x_{2}}\right|^{q}\right] \\
\leq & C_{q}\left(\left|x_{1}-x_{2}\right|^{q}+\left|s_{1}-s_{2}\right|^{q / 2}+\left|t_{1}-t_{2}\right|^{q / 2}\right. \\
& E\left[\left|\int_{s_{2}}^{t_{1}}\left(b_{n}\left(u, X_{u}^{n, s_{1}, x_{1}}\right)-b_{n}\left(u, X_{u}^{n, s_{1}, x_{2}}\right)\right) d u\right|^{q}\right] \\
& \left.+E\left[\left|\int_{s_{2}}^{t_{1}}\left(b_{n}\left(u, X_{u}^{n, s_{1}, x_{2}}\right)-b_{n}\left(u, X_{u}^{n, s_{2}, x_{2}}\right)\right) d u\right|^{q}\right]\right) .
\end{aligned}
$$

Using the fact that $X_{t}^{n,, s}$ is a stochastic flow of diffeomorphisms (see e.g. [22]), the mean value theorem and Proposition 3.7, we get

$$
\begin{aligned}
& E\left[\left|\int_{s_{2}}^{t_{1}}\left(b_{n}\left(u, X_{u}^{n, s_{1}, x_{1}}\right)-b_{n}\left(u, X_{u}^{n, s_{1}, x_{2}}\right)\right) d u\right|^{q}\right] \\
= & \left|x_{1}-x_{2}\right|^{q} E\left[\left|\int_{s_{2}}^{t_{1}} \int_{0}^{1}\left(b_{n}^{\prime}\left(u, X_{u}^{n, s_{1}, x_{1}+\tau\left(x_{2}-x_{1}\right)}\right) \frac{\partial}{\partial x} X_{u}^{n, s_{1}, x_{1}+\tau\left(x_{2}-x_{1}\right)}\right) d \tau d u\right|^{q}\right] \\
\leq & \left|x_{1}-x_{2}\right|^{q} \int_{0}^{1} E\left[\left|\int_{s_{2}}^{t_{1}}\left(b_{n}^{\prime}\left(u, X_{u}^{n, s_{1}, x_{1}+\tau\left(x_{2}-x_{1}\right)}\right) \frac{\partial}{\partial x} X_{u}^{n, s_{1}, x_{1}+\tau\left(x_{2}-x_{1}\right)}\right) d u\right|^{q}\right] d \tau \\
= & \left|x_{1}-x_{2}\right|^{q} \int_{0}^{1} E\left[\left|\frac{\partial}{\partial x} X_{t_{1}}^{n, s_{1}, x_{1}+\tau\left(x_{2}-x_{1}\right)}-\frac{\partial}{\partial x} X_{s_{2}}^{n, s_{1}, x_{1}+\tau\left(x_{2}-x_{1}\right)}\right|^{q}\right] d \tau \\
\leq & C_{q}\left|x_{1}-x_{2}\right|^{q} \sup _{t \in\left[s_{1}, 1\right], x \in \mathbb{R}^{d}} E\left[\left|\frac{\partial}{\partial x} X_{t_{1}}^{n, s_{1}, x}\right|^{q}\right] \\
\leq & C_{d, q}\left(\|b\|_{\infty}\right)\left|x_{1}-x_{2}\right|^{q} .
\end{aligned}
$$


Finally we observe that estimation of the last term of the right hand side of (3.18) can be reduced to the previous case (3.19) by applying the Markov property, since

$$
\begin{aligned}
& E\left[\left|\int_{s_{2}}^{t_{1}}\left(b_{n}\left(u, X_{u}^{n, s_{1}, x_{2}}\right)-b_{n}\left(u, X_{u}^{n, s_{2}, x_{2}}\right)\right) d u\right|^{q}\right] \\
\leq & \int_{s_{2}}^{t_{1}} E\left[\left|b_{n}\left(u, X_{u}^{n, s_{1}, x_{2}}\right)-b_{n}\left(u, X_{u}^{n, s_{2}, x_{2}}\right)\right|^{q}\right] d u \\
= & \int_{s_{2}}^{t_{1}} E\left[\left.E\left[\left|b_{n}\left(u, X_{u}^{n, s_{2}, y}\right)-b_{n}\left(u, X_{u}^{n, s_{2}, x_{2}}\right)\right|^{q}\right]\right|_{\left.y=X_{s_{2}}^{n, s_{1}, x_{2}}\right] d u}\right. \\
\leq & C E\left[\left|X_{s_{2}}^{n, s_{1}, x_{2}}-x_{2}\right|^{q}\right]=C E\left[\left|X_{s_{2}}^{n, s_{1}, x_{2}}-X_{s_{1}}^{n, s_{1}, x_{2}}\right|^{q}\right] \\
\leq & M_{q}\left|s_{2}-s_{1}\right|^{q / 2}
\end{aligned}
$$

for a positive constant $M_{q}<\infty$.

Therefore, we have

$$
E\left[\left|X_{t_{1}}^{n, s_{1}, x_{1}}-X_{t_{2}}^{n, s_{2}, x_{2}}\right|^{q}\right] \leq C_{q}\left(\left|s_{1}-s_{2}\right|^{q / 2}+\left|t_{1}-t_{2}\right|^{q / 2}+\left|x_{1}-x_{2}\right|^{q}\right)
$$

for a constant $C_{q}$ independent of $n$.

To complete the proof, we use the fact that $X_{t_{1}}^{n, s_{1}, x_{1}} \rightarrow X_{t_{1}}^{s_{1}, x_{1}}$ and $X_{t_{2}}^{n, s_{2}, x_{2}} \rightarrow X_{t_{2}}^{s_{2}, x_{2}}$ in $L^{2}(\mu)$ for $n \longrightarrow \infty$ (see [26]) together with Fatou's lemma applied to a.e. convergent subsequences of $\left\{X_{t_{1}}^{n, s_{1}, x_{1}}\right\}_{n=1}^{\infty}$ and $\left\{X_{t_{2}}^{n, s_{2}, x_{2}}\right\}_{n=1}^{\infty}$.

This concludes step one of our program.

We now proceed to Step 2. For simplicity we consider $s=0$, i.e. we look at the sequence $\left\{X_{t}^{n, x}\right\}_{n \geq 1}:=\left\{X_{t}^{n, 0, x}\right\}_{n \geq 1}$ and $X_{t}^{x}:=X_{t}^{0, x}$. The following lemma establishes convergence of the above sequence:

Lemma 3.14 For any $\varphi \in C_{0}^{\infty}\left(\mathbb{R}^{d} ; \mathbb{R}^{d}\right)$ and $t \in[0,1]$ the sequence

$$
\left\langle X_{t}^{n}, \varphi\right\rangle=\int_{\mathbb{R}^{d}}\left\langle X_{t}^{n, x}, \varphi(x)\right\rangle_{\mathbb{R}^{d}} d x
$$

converges to $\left\langle X_{t}, \varphi\right\rangle$ in $L^{2}(\Omega, \mu)$.

Proof. Denote by $D_{s}$ the Malliavin derivative (see the Appendix) and by $U$ the compact support of $\varphi$. By noting the inequalities

$$
\begin{aligned}
& E\left[\left|D_{s}\left\langle X_{t}^{n}, \varphi\right\rangle\right|^{2}\right]=E\left[\left|\left\langle D_{s} X_{t}^{n}, \varphi\right\rangle\right|^{2}\right] \\
& \leq\|\varphi\|_{L^{2}\left(\mathbb{R}^{d}\right)}^{2}|U| \sup _{x \in U} E\left[\left|D_{s} X_{t}^{n, x}\right|^{2}\right]
\end{aligned}
$$

and

$$
\begin{gathered}
E\left[\left|D_{s}\left\langle X_{t}^{n}, \varphi\right\rangle_{L^{2}\left(\mathbb{R}^{d}\right)}-D_{s^{\prime}}\left\langle X_{t}^{n}, \varphi\right\rangle\right|^{2}\right] \\
=E\left[\left|\left\langle D_{s} X_{t}^{n}-D_{s^{\prime}} X_{t}^{n}, \varphi\right\rangle\right|^{2}\right] \\
\leq\|\varphi\|_{L^{2}\left(\mathbb{R}^{d}\right)}^{2}|U| \sup _{x \in U} E\left[\left|D_{s} X_{t}^{n, x}-D_{s^{\prime}} X_{t}^{n, x}\right|^{2}\right]
\end{gathered}
$$

we can invoke Corollary 3.33 together with Lemma $3.5[26]$ to obtain a subsequence $\left\langle X_{t}^{n(k)}, \varphi\right\rangle$ converging in $L^{2}(\Omega, \mu)$ as $k \rightarrow \infty$. Denote the limit by $Y(\varphi)$. 
Similary to the proof of Lemma 3.11 one can show that $E\left[\left\langle X_{t}^{n}, \varphi\right\rangle \mathcal{E}\left(\int_{0}^{1} h(u) d B_{u}\right)\right]$ converges to $E\left[\left\langle X_{t}, \varphi\right\rangle \mathcal{E}\left(\int_{0}^{1} h(u) d B_{u}\right)\right]$ for all $h \in C_{b}^{1}\left(\mathbb{R} ; \mathbb{R}^{d}\right)$. We then get that $\left\langle X_{t}^{n}, \varphi\right\rangle$ converges weakly to $\left\langle X_{t}, \varphi\right\rangle$, and so by uniqueness of the limits we can conclude that

$$
Y(\varphi)=\left\langle X_{t}, \varphi\right\rangle \text {. }
$$

To see that the full sequence converges we assume that there exists an $\epsilon>0$ and a subsequence $\left\langle X_{t}^{n(k)}, \varphi\right\rangle$ such that

$$
\left\|\left\langle X_{t}^{n(k)}, \varphi\right\rangle-\left\langle X_{t}, \varphi\right\rangle\right\| \geq \epsilon
$$

for every $k$. Applying the above procedure to $\left\langle X_{t}^{n(k)}, \varphi\right\rangle$ gives a further subsequence converging to $\left\langle X_{t}, \varphi\right\rangle$ thus giving a contradiction.

We are now able to finalize the proof of Proposition 3.6.

\section{Proof of Proposition 3.6.}

Using Proposition 3.7, we have

$$
\sup _{n} E\left[\left|\frac{\partial}{\partial x} X_{t}^{n, x}\right|^{p}\right]<\infty
$$

Hence there exists a subsequence of $\frac{\partial}{\partial x} X_{t}^{n(k), x}$ converging in the weak topology of $L^{2}\left(\Omega, L^{p}(U)\right)$ to an element $Y$. Then we have for any $A \in \mathcal{F}$ and $\varphi \in C_{0}^{\infty}\left(U ; \mathbb{R}^{d}\right)$

$$
\begin{gathered}
E\left[1_{A}\left\langle X_{t}, \varphi^{\prime}\right\rangle\right]=\lim _{k \rightarrow \infty} E\left[1_{A}\left\langle X_{t}^{n(k)}, \varphi^{\prime}\right\rangle\right] \\
=\lim _{k \rightarrow \infty}-E\left[1_{A}\left\langle\frac{\partial}{\partial x} X_{t}^{n(k)}, \varphi\right\rangle\right]=-E\left[1_{A}\langle Y, \varphi\rangle\right] .
\end{gathered}
$$

Hence we have for $\varphi \in C_{0}^{\infty}$ :

$$
\left\langle X_{t}, \varphi^{\prime}\right\rangle=-\langle Y, \varphi\rangle
$$

$P$-a.s.. Finally, we need to show that there exists a measurable set $\Omega_{0} \subset \Omega$ with full measure such that $X_{t}$ has a weak derivative on this subset. To this end choose a sequence $\left\{\varphi_{n}\right\}$ in $C^{\infty}\left(U ; \mathbb{R}^{d}\right)$ dense in $W_{0}^{1,2}\left(U ; \mathbb{R}^{d}\right)$. Choose a measurable subset $\Omega_{n}$ of $\Omega$ with full measure such that (3.20) holds on $\Omega_{n}$ with $\varphi$ replaced by $\varphi_{n}$. Then $\Omega_{0}:=\cap_{n \geq 1} \Omega_{n}$ satisfies the desired property.

We now return to the weighted Sobolev spaces. Using the same techniques as in the above lemma, we prove the following

Lemma 3.15 For all $p \in(1, \infty)$ we have

$$
X_{t} \in L^{2}\left(\Omega, W^{1, p}\left(\mathbb{R}^{d}, w\right)\right)
$$

Proof. For simplicity, we consider the case $d=1$. It suffices to show that $E\left[\left(\int\left|\frac{\partial}{\partial x} X_{t}^{x}\right|^{p} w(x) d x\right)^{2 / p}\right]$ is finite. To this end, let $X_{t}^{n, x}$ denote the sequence approximating $X_{t}^{x}$ as in the previous lemma. Assume first that $p \geq 2$. Then by Hölder's inequality w.r.t. the Wiener measure $\mu$ we have

$$
E\left[\left(\int\left|\frac{\partial}{\partial x} X_{t}^{n, x}\right|^{p} w(x) d x\right)^{2 / p}\right]
$$




$$
\leq\left(E\left[\int\left|\frac{\partial}{\partial x} X_{t}^{n, x}\right|^{p} w(x) d x\right)^{2 / p} \leq\left(\int w(x) d x\right)^{p / 2}\left(\sup _{x \in \mathbb{R}} E\left|\frac{\partial}{\partial x} X_{t}^{n, x}\right|^{p}\right)^{2 / p} .\right.
$$

For $1<p \leq 2$, by Hölder's inequality w.r.t. $w(x) d x$ we have

$$
E\left[\left(\int\left|\frac{\partial}{\partial x} X_{t}^{n, x}\right|^{p} w(x) d x\right)^{2 / p}\right] \leq\left(\int w(x) d x\right)^{(4-p) / 2} \sup _{x \in \mathbb{R}^{d}} E\left[\left|\frac{\partial}{\partial x} X_{t}^{n, x}\right|^{2}\right] .
$$

In both cases we can find a subsequence converging to an element $Y \in L^{2}\left(\Omega, L^{p}\left(\mathbb{R}^{d}, w\right)\right)$ in the weak topology, in particular for every $A \in \mathcal{F}$ and $f \in L^{q}\left(\mathbb{R}^{d}, w\right)(q$ is the Sobolev conjugate of $p$ ) we have

$$
\lim _{k \rightarrow \infty} E\left[1_{A} \int \frac{\partial}{\partial x} X_{t}^{n(k), x} f(x) w(x) d x\right]=E\left[1_{A} \int Y(x) f(x) w(x) d x\right]
$$

by choosing $f$ such that $f w \in L^{q}(\mathbb{R}, d x)$ (e.g. put $f(x)=e^{-w(x)} \varphi(x)$ for $\varphi \in C_{0}^{\infty}(\mathbb{R})$ ), it follows that $Y$ must coincide with the weak derivative of $X_{t}^{x}$. This proves the lemma.

We now complete the proof of our main theorem in this section (Theorem 3.3) and its corollary:

Proof of Theorem 3.3. Denote by $\mathbb{R} \times \mathbb{R} \times \mathbb{R}^{d} \ni(s, t, x) \longmapsto \phi_{s, t}(x) \in \mathbb{R}^{d}$ the continuous version of the solution map $(s, t, x) \longmapsto X_{t}^{s, x}$ provided by Corollary 3.13. Let $\Omega^{*}$ be the set of all $\omega \in \Omega$ such that the SDE (3.7) has a unique spatially Sobolev differentiable family of solutions. Then by completeness of the probability space $(\Omega, \mathcal{F}, \mu)$, it follows that $\Omega^{*} \in \mathcal{F}$ and $\mu\left(\Omega^{*}\right)=1$. Furthermore, by uniqueness of solutions of the SDE (3.7), it is easy to check that the following two-parameter group property

$$
\phi_{s, t}(\cdot, \omega)=\phi_{u, t}(\cdot, \omega) \circ \phi_{s, u}(\cdot, \omega), \quad \phi_{s, s}(x, \omega)=x,
$$

holds for all $s, u, t \in \mathbb{R}$, all $x \in \mathbb{R}^{d}$ and all $\omega \in \Omega^{*}$. Finally, we apply Lemma 3.15 and use the relation $\phi_{s, t}(\cdot, \omega)=\phi_{t, s}^{-1}(\cdot, \omega)$, to complete the proof of the theorem.

Proof of Corollary 3.5. Let $\Omega^{*}$ denote the set of full Wiener measure introduced in the above proof of Theorem 3.3. We claim that $\theta(t, \cdot)\left(\Omega^{*}\right)=\Omega^{*}$ for all $t \in \mathbb{R}$. To see this, let $\omega \in \Omega^{*}$ and fix an arbitrary $t_{1} \in \mathbb{R}$. Then from the autonomous SDE (3.11) it follows that

$$
X_{t+t_{1}}^{t_{1}, x}(\omega)=x+\int_{t_{1}}^{t+t_{1}} b\left(X_{u}^{t_{1}, x}(\omega)\right) d u+B_{t+t_{1}}(\omega)-B_{t_{1}}(\omega), t_{1}, t \in \mathbb{R},
$$

By the helix property of $B$ and a simple change of variable the above relation implies

$$
X_{t+t_{1}}^{t_{1}, x}(\omega)=x+\int_{0}^{t} b\left(X_{u+t_{1}}^{t_{1}, x}(\omega)\right) d u+B_{t}\left(\theta\left(t_{1}(\omega)\right)\right), \quad t \in \mathbb{R},
$$

The above relation implies that the SDE (3.11) admits a Sobolev differentiable family of solutions when $\omega$ is replaced by $\theta\left(t_{1}, \omega\right)$. Hence $\theta\left(t_{1}, \omega\right) \in \Omega^{*}$. Thus $\theta\left(t_{1}, \cdot\right)\left(\Omega^{*}\right) \subseteq \Omega^{*}$, and since $t_{1} \in \mathbb{R}$ is arbitrary, this proves our claim. Furthermore, using uniqueness in the integral equation (3.22) it follows that

$$
X_{t_{2}+t_{1}}^{t_{1}, x}(\omega)=X_{t_{2}}^{0, x}\left(\theta\left(t_{1}, \omega\right)\right)
$$


for all $t_{1}, t_{2} \in \mathbb{R}$, all $x \in \mathbb{R}^{d}$ and $\omega \in \Omega^{*}$. To prove the following cocycle property for all $\omega \in \Omega^{*}$

$$
\phi_{0, t_{1}+t_{2}}(\cdot, \omega)=\phi_{0, t_{2}}\left(\cdot, \theta\left(t_{1}, \omega\right)\right) \circ \phi_{0, t_{1}}(\cdot, \omega)
$$

we rewrite the identity (3.24) in the form

$$
\phi_{t_{1}, t_{1}+t_{2}}(x, \omega)=\phi_{0, t_{2}}\left(x, \theta\left(t_{1}, \omega\right)\right), t_{1}, t_{2} \in \mathbb{R}, x \in \mathbb{R}^{d}, \omega \in \Omega^{*},
$$

replace $x$ by $\phi_{0, t_{1}}(x, \omega)$ in the above identity and invoke the two-parameter flow property (3.21). This completes the proof of Corollary 3.5.

Finally, we give an extension of Theorem 3.3 to a class of non-degenerate $d$-dimensional Itô-diffusions.

Theorem 3.16 Consider the time-homogeneous $\mathbb{R}^{d}$-valued SDE

$$
d X_{t}^{x}=b\left(X_{t}^{x}\right) d t+\sigma\left(X_{t}^{x}\right) d B_{t}, X_{0}^{x}=x \in \mathbb{R}^{d}, 0 \leq t \leq 1,
$$

where the coefficients $b: \mathbb{R}^{d} \longrightarrow \mathbb{R}^{d}$ and $\sigma: \mathbb{R}^{d} \longrightarrow \mathbb{R}^{d} \times \mathbb{R}^{d}$ are Borel measurable. Suppose that $\sigma(x)$ has an inverse $\sigma^{-1}(x)$ for all $x \in \mathbb{R}^{d}$. Further assume that $\sigma^{-1}: \mathbb{R}^{d} \longrightarrow \mathbb{R}^{d} \times \mathbb{R}^{d}$ is continuously differentiable such that

$$
\frac{\partial}{\partial x_{k}} \sigma_{l j}^{-1}=\frac{\partial}{\partial x_{j}} \sigma_{l k}^{-1}
$$

for all $l, k, j=1, \ldots, d$. In addition, require that the function $\Lambda: \mathbb{R}^{d} \longrightarrow \mathbb{R}^{d}$ defined by

$$
\Lambda(x):=\int_{0}^{1} \sigma^{-1}(t x) \cdot x d t
$$

possesses a Lipschitz continuous inverse $\Lambda^{-1}: \mathbb{R}^{d} \longrightarrow \mathbb{R}^{d}$. Let $D \Lambda: \mathbb{R}^{d} \longrightarrow L\left(\mathbb{R}^{d}, \mathbb{R}^{d}\right)$ and $D^{2} \Lambda: \mathbb{R}^{d} \longrightarrow L\left(\mathbb{R}^{d} \times \mathbb{R}^{d}, \mathbb{R}^{d}\right)$ be the existing corresponding derivatives of $\Lambda$.

Assume that the function $b^{*}: \mathbb{R}^{d} \longrightarrow \mathbb{R}^{d}$ given by

$$
\begin{aligned}
& b^{*}(x):=D \Lambda\left(\Lambda^{-1}(x)\right)\left[b\left(\Lambda^{-1}(x)\right)\right] \\
& +\frac{1}{2} D^{2} \Lambda\left(\Lambda^{-1}(x)\right)\left[\sum_{i=1}^{d} \sigma\left(\Lambda^{-1}(x)\right)\left[e_{i}\right], \sum_{i=1}^{d} \sigma\left(\Lambda^{-1}(x)\right)\left[e_{i}\right]\right]
\end{aligned}
$$

is bounded and Borel measurable, where $e_{i}, i=1, \ldots, d$, is a basis of $\mathbb{R}^{d}$.

Then there exists a stochastic flow $(s, t, x) \longmapsto \phi_{s, t}(x)$ of the $S D E$ (3.26) such that

$$
\phi_{s, t}(\cdot) \in L^{2}\left(\Omega, W^{p}\left(\mathbb{R}^{d}, w\right)\right)
$$

for all $0 \leq s \leq t \leq 1$ and all $p>1$.

Proof. Because of our assumptions we see that $\Lambda^{-1}$ is twice continuously differentiable and that

$$
D \Lambda(y) \sigma(y)=\mathcal{I}_{d}
$$

for all $y \in \mathbb{R}^{d}$. 
Then Itô's Lemma applied to (3.7) implies that

$$
\begin{aligned}
d Y_{t}^{x}= & D \Lambda\left(\Lambda^{-1}\left(Y_{t}^{x}\right)\right)\left[b\left(\Lambda^{-1}\left(Y_{t}^{x}\right)\right)\right] \\
& +\frac{1}{2} D^{2} \Lambda\left(\Lambda^{-1}\left(Y_{t}^{x}\right)\right)\left[\sum_{i=1}^{d} \sigma\left(\Lambda^{-1}\left(Y_{t}^{x}\right)\right)\left[e_{i}\right], \sum_{i=1}^{d} \sigma\left(\Lambda^{-1}\left(Y_{t}^{x}\right)\right)\left[e_{i}\right]\right] d t+d B_{t}, \\
Y_{0}^{x}= & \Lambda(x), 0 \leq t \leq 1,
\end{aligned}
$$

where $Y_{t}^{x}=\Lambda\left(X_{t}^{x}\right)$. Because of Theorem 3.3 and a chain rule for functions in Sobolev spaces (see e.g. [40]) there exists a stochastic flow $(s, t, x) \longmapsto \phi_{s, t}(x)$ of the SDE (3.26) such that $\phi_{s, t}(\cdot) \in L^{2}\left(\Omega, W^{p}\left(\mathbb{R}^{d}, w\right)\right)$ for all $0 \leq s \leq t \leq 1$ and all $p>1$.

\subsection{Application to the Stochastic Transport Equation}

In this section we will study the stochastic transport equation

$$
\left\{\begin{array}{l}
d_{t} u(t, x)+(b(t, x) \cdot D u(t, x)) d t+\sum_{i=1}^{d} e_{i} \cdot D u(t, x) \circ d B_{t}^{i}=0 \\
u(0, x)=u_{0}(x)
\end{array}\right.
$$

where $e_{1}, \ldots e_{d}$ is the canonical basis of $\mathbb{R}^{d}, b:[0,1] \times \mathbb{R}^{d} \rightarrow \mathbb{R}^{d}$ is a given bounded measurable vector field and $u_{0}: \mathbb{R}^{d} \rightarrow \mathbb{R}$ is a given initial data. The stochastic integration is understood in the Stratonovich sense.

In [22] it is proved that for smooth data and sufficiently regular vector field $b,(3.27)$ has an explicit solution $u(t, x)=u_{0}\left(\phi_{t}^{-1}(x)\right)$ where $\phi_{t}(x)$ is the flow map generated by the strong solutions $\left(X_{t}^{x}\right)_{t>0}$ of the SDE (3.7). In fact this solution of the transport equation is strong in the sense that $u(t, \cdot)$ is differentiable everywhere in $x$ almost surely for all $t$, and it satisfies the integral equation

$$
u(t, x)+\int_{0}^{t} D u(s, x) \cdot b(s, x) d s+\sum_{i=1}^{d} \int_{0}^{t} e_{i} \cdot D u(s, x) \circ d B_{s}^{i}=u_{0}(x)
$$

almost surely, for every $t$.

We shall use the following notion of weak solution (cf. Definition 12 in [14]).

Definition 3.17 Let $b$ be bounded and measurable and $u_{0} \in L^{\infty}\left(\mathbb{R}^{d}\right)$. A differentiable, weak $L^{\infty}$-solution of the transport equation (3.27) is a stochastic process $u \in L^{\infty}(\Omega \times$ $\left.[0,1] \times \mathbb{R}^{d}\right)$ such that, for every $t$, the function $u(t, \cdot)$ is weakly differentiable a.s. with $\sup _{0 \leq s \leq 1, x \in \mathbb{R}^{d}} E\left[|D u(s, x)|^{4}\right]<\infty$ and for every test function $\theta \in C_{0}^{\infty}\left(\mathbb{R}^{d}\right)$, the process $\int_{\mathbb{R}^{d}} \theta(x) u(t, x) d x$ has a continuous modification which is an $\mathcal{F}_{t}$-semi-martingale and

$$
\begin{aligned}
\int_{\mathbb{R}^{d}} \theta(x) u(t, x) d x & =\int_{\mathbb{R}^{d}} \theta(x) u_{0}(x) d x \\
& -\int_{0}^{t} \int_{\mathbb{R}^{d}} D u(s, x) \cdot b(s, x) \theta(x) d x d s \\
& +\sum_{i=1}^{d} \int_{0}^{t}\left(\int_{\mathbb{R}^{d}} u(s, x) D_{i} \theta(x) d x\right) \circ d B_{s}^{i},
\end{aligned}
$$

where $D u(t, x)$ is the weak derivative of $u(t, x)$ in the space-variable. 
Our definition of weak solution differs slightly from that in [14] due to the fact that we do not require any regularity on the coefficient $b$ except Borel measurability and boundedness. To compensate for it, the expression depends on the weak derivative of $u(t, x)$.

It is easy to see that equation (3.28) can be written in the equivalent Itô form:

Lemma 3.18 A process $u \in L^{\infty}\left(\Omega \times[0,1] \times \mathbb{R}^{d}\right)$ is a differentiable, weak $L^{\infty}$ solution of the transport equation (3.27) if and only if, for every test function $\theta \in C_{0}^{\infty}\left(\mathbb{R}^{d}\right)$, the process $\int_{\mathbb{R}^{d}} \theta(x) u(t, x) d x$ has a continuous $\mathcal{F}_{t^{-} \text {-adapted modification and }}$

$$
\begin{aligned}
\int_{\mathbb{R}^{d}} \theta(x) u(t, x) d x & =\int_{\mathbb{R}^{d}} \theta(x) u_{0}(x) d x \\
& -\int_{0}^{t} \int_{\mathbb{R}^{d}} D u(s, x) \cdot b(s, x) \theta(x) d x d s \\
& +\sum_{i=1}^{d} \int_{0}^{t}\left(\int_{\mathbb{R}^{d}} u(s, x) D_{i} \theta(x) d x\right) d B_{s}^{i} \\
& +\frac{1}{2} \int_{0}^{t} \int_{\mathbb{R}^{d}} u(s, x) \Delta \theta(x) d x d s .
\end{aligned}
$$

The main result of this section is the following existence and uniqueness theorem for solutions of the stochastic transport equation (3.27):

Theorem 3.19 Let $b$ be bounded and Borel measurable. Suppose $u_{0} \in C_{b}^{1}\left(\mathbb{R}^{d}\right)$. Then there exists a unique $W^{1, \infty}$ weak solution $u(t, x)$ to the stochastic transport equation (3.27). (Moreover, for fixed $t$ and $x$, this solution is Malliavin-differentiable.)

Remark 3.20 As noted in [14], the deterministic transport equation is generally ill-posed under the conditions of Theorem 3.19. It is remarkable that Brownian forcing on the transport equation induces uniqueness and regularity of the solution.

We shall prove Theorem 3.19 using a sequence $b_{n}:[0,1] \times \mathbb{R}^{d} \rightarrow \mathbb{R}^{d}$ of uniformly bounded sequence of smooth functions with compact support converging almost everywhere to $b$. We then study the corresponding sequence of solutions of the transport equation (3.27) when $b$ is replaced by $b_{n}$.

For the rest of this section we denote by $\phi_{t}$ the flow of the SDE (3.7) driven by the vector field $b$, and by $\phi_{n, t}$ the flow of the $\operatorname{SDE}(3.7)$ with $b_{n}$ in place of $b$.

We begin with the following lemma:

Lemma 3.21 Let $u_{0} \in C_{b}^{1}\left(\mathbb{R}^{d}\right)$ and $f \in L^{1}\left(\mathbb{R}^{d}\right)$. Then the sequence

$$
\left(\int_{\mathbb{R}^{d}} u_{0}\left(\phi_{n, s}^{-1}(x)\right) f(x) d x\right)_{n \geq 1}
$$

converges to $\int_{\mathbb{R}^{d}} u_{0}\left(\phi_{s}^{-1}(x)\right) f(x) d x$ in $L^{2}(\Omega)$ for every $s \in[0,1]$.

Proof. Consider

$$
\left\|\int_{\mathbb{R}^{d}} u_{0}\left(\phi_{n, s}^{-1}(x)\right) f(x) d x-\int_{\mathbb{R}^{d}} u_{0}\left(\phi_{s}^{-1}(x)\right) f(x) d x\right\|_{L^{2}(\Omega)}
$$




$$
\leq \int_{\mathbb{R}^{d}}\left\|u_{0}\left(\phi_{n, s}^{-1}(x)\right)-u_{0}\left(\phi_{s}^{-1}(x)\right)\right\|_{L^{2}(\Omega)}|f(x)| d x
$$

We have $\left\|u_{0}\left(\phi_{n, s}^{-1}(x)\right)-u_{0}\left(\phi_{s}^{-1}(x)\right)\right\|_{L^{2}(\Omega)} \leq\left\|D u_{0}\right\|_{\infty}\left\|\phi_{n, s}^{-1}(x)-\phi_{s}^{-1}(x)\right\|_{L^{2}(\Omega)}$ which goes to zero for every $s$ and $x$. Now

$$
\left\|u_{0}\left(\phi_{n, s}^{-1}\right)-u_{0}\left(\phi_{s}^{-1}\right)\right\|_{L^{2}(\Omega)}|f| \leq 2\left\|u_{0}\right\|_{\infty}|f| \in L^{1}\left(\mathbb{R}^{d}\right)
$$

and the result follows by dominated convergence.

We also need the following result (see Theorem 2 in [17] and also [35], [36]):

Theorem 3.22 Let $\mathcal{U}$ be open subset of $\mathbb{R}^{d}$ and $f \in W^{1, d}(\mathcal{U})$ be a homeomorphism. Then $f$ satisfies the Lusin's condition, that is

$$
E \subset \mathcal{U},|E|=0 \Longrightarrow|f(E)|=0 .
$$

Here $|A|$ stands for the Lebesgue measure of a set $A$.

Moreover, for every measurable function $g: \mathcal{U} \longrightarrow[0, \infty)$ and measurable set $E \subset \mathcal{U}$ the following change of variable formula is valid:

$$
\int_{E}(g \circ f)|\operatorname{det} J f| d x=\int_{f(E)} g(y) d y
$$

where det $J f$ is the determinant of the Jacobian of $f$.

Remark 3.23 The random diffeomorphisms $\phi_{t}(\cdot), \phi_{t}^{-1}(\cdot) \in W_{\text {loc }}^{1, p}\left(\mathbb{R}^{d}\right)$ a.e. satisfy the conditions of Theorem 3.22 on each bounded and open subset $\mathcal{U}$ of $\mathbb{R}^{d}$.

We are now ready to prove Theorem 3.19:

\section{Proof of Theorem 3.19.}

\section{Existence of a weak solution:}

We consider the approximation $\left\{b_{n}\right\}$ of $b$ as described in Corollary 13. Then we know that there exists a unique strong solution to the transport equation (3.27) when $b$ is replaced by $b_{n}$, which is uniquely given by $u_{n}(t, x)=u_{0}\left(\phi_{n, t}^{-1}(x)\right), n \geq 1$. In particular, $u_{n}$ is a differentiable, weak $L^{\infty}$-solution, such that for every $\theta \in C^{\infty}\left(\mathbb{R}^{d}\right)$

$$
\begin{aligned}
\int_{\mathbb{R}^{d}} \theta(x) u_{n}(t, x) d x & =\int_{\mathbb{R}^{d}} \theta(x) u_{0}(x) d x \\
& -\int_{0}^{t} \int_{\mathbb{R}^{d}} D u_{n}(s, x) \cdot b_{n}(s, x) \theta(x) d x d s \\
& +\sum_{i=1}^{d} \int_{0}^{t}\left(\int_{\mathbb{R}^{d}} u_{n}(s, x) D_{i} \theta(x) d x\right) d B_{s}^{i} \\
& +\frac{1}{2} \int_{0}^{t} \int_{\mathbb{R}^{d}} u_{n}(s, x) \Delta \theta(x) d x d s .
\end{aligned}
$$


Let's now define $u(t, x):=u_{0}\left(\phi_{t}^{-1}(x)\right)$ so that $u \in L^{\infty}\left(\Omega \times[0,1] \times \mathbb{R}^{d}\right)$, and $u(t, \cdot)$ is weakly differentiable, a.s. We now let $n$ go to infinity to get that $u(t, x)$ is a solution of the transport equation.

The following two limits exist in $L^{2}(\Omega)$ by Lemma 3.21 and dominated convergence:

$$
\begin{aligned}
\int_{\mathbb{R}^{d}} \theta(x) u_{n}(t, x) d x & \rightarrow \int_{\mathbb{R}^{d}} \theta(x) u(t, x) d x \\
\int_{0}^{t} \int_{\mathbb{R}^{d}} u_{n}(s, x) \Delta \theta(x) d x d s & \rightarrow \int_{0}^{t} \int_{\mathbb{R}^{d}} u(s, x) \Delta \theta(x) d x d s
\end{aligned}
$$

By the Itô isometry we have

$$
\sum_{i=1}^{d} \int_{0}^{t}\left(\int_{\mathbb{R}^{d}} u_{n}(s, x) D_{i} \theta(x) d x\right) d B_{s}^{i} \rightarrow \sum_{i=1}^{d} \int_{0}^{t}\left(\int_{\mathbb{R}^{d}} u(s, x) D_{i} \theta(x) d x\right) d B_{s}^{i}
$$

in $L^{2}(\Omega)$. Finally, we claim that

$$
\int_{0}^{t} \int_{\mathbb{R}^{d}} D u_{n}(s, x) \cdot b_{n}(s, x) \theta(x) d x d s \rightarrow \int_{0}^{t} \int_{\mathbb{R}^{d}} D u(s, x) \cdot b(s, x) \theta(x) d x d s
$$

in $L^{2}(\Omega)$. To see this observe that

$$
\left(\int_{0}^{t} \int_{\mathbb{R}^{d}} D u_{n}(s, x) \cdot b_{n}(s, x) \theta(x) d x d s\right)_{n}
$$

is convergent in $L^{2}(\Omega)$ because of the convergence of the other terms in equality (3.29). Then the claim is proved once we show that $\int_{0}^{t} \int_{\mathbb{R}^{d}} D u_{n}(s, x) \cdot b_{n}(s, x) \theta(x) d x d s$ converges weakly to $\int_{0}^{t} \int_{\mathbb{R}^{d}} D u(s, x) \cdot b(s, x) \theta(x) d x d s$. Then the strong and weak limit must coincide.

To prove weak convergence, we write the difference in three parts, namely:

$$
\begin{gathered}
\int_{0}^{t} \int_{\mathbb{R}^{d}} D u_{n}(s, x) \cdot b_{n}(s, x) \theta(x) d x d s-\int_{0}^{t} \int_{\mathbb{R}^{d}} D u(s, x) \cdot b(s, x) \theta(x) d x d s \\
=\int_{0}^{t} \int_{\mathbb{R}^{d}} D u_{n}(s, x) \cdot b_{n}(s, x) \theta(x) d x d s-\int_{0}^{t} \int_{\mathbb{R}^{d}} D u_{n}(s, x) \cdot b(s, x) \theta(x) d x d s \\
+\int_{0}^{t} \int_{\mathbb{R}^{d}} D u_{0}\left(\phi_{n, s}^{-1}(x)\right) D \phi_{n, s}^{-1}(x) \cdot b(s, x) \theta(x) d x d s-\int_{0}^{t} \int_{\mathbb{R}^{d}} D u_{0}\left(\phi_{s}^{-1}(x)\right) D \phi_{n, s}^{-1}(x) \cdot b(s, x) \theta(x) d x d s \\
+\int_{0}^{t} \int_{\mathbb{R}^{d}} D u_{0}\left(\phi_{s}^{-1}(x)\right) D \phi_{n, s}^{-1}(x) \cdot b(s, x) \theta(x) d x d s-\int_{0}^{t} \int_{\mathbb{R}^{d}} D u_{0}\left(\phi_{s}^{-1}(x)\right) D \phi_{s}^{-1}(x) \cdot b(s, x) \theta(x) d x d s \\
=(i)_{n}+(i i)_{n}+(i i i)_{n}
\end{gathered}
$$

We shall deal with these terms separately.

$(\alpha)$ : The first term $(i)_{n}$ converges to 0 strongly in $L^{2}(\Omega)$ as $n \rightarrow \infty$, since by Hölder's inequality and Fubini's theorem

$$
E\left[(i)_{n}^{2}\right]=E\left[\left(\int_{0}^{t} \int_{\mathbb{R}^{d}} D u_{n}(s, x) \cdot\left(b_{n}(s, x)-b(s, x)\right) \theta(x) d x d s\right)^{2}\right]
$$




$$
\leq \int_{0}^{t} \int_{\mathbb{R}^{d}} E\left[\left|D u_{n}(s, x)\right|^{2}\right]\left|b_{n}(s, x)-b(s, x)\right|^{2}|\theta(x)| d x\|\theta\|_{L^{1}(\mathbb{R})}
$$

We have that

$$
E\left[\left|D u_{n}(s, x)\right|^{2}\right] \leq\left\|D u_{0}\right\|_{\infty}^{2} E\left[\left|D \phi_{n, s}^{-1}(x)\right|^{2}\right]
$$

which is uniformly bounded in $n, s$ and $x$ by Proposition 3.7. Then, using dominated convergence, we obtain $\lim _{n \rightarrow \infty}(i)_{n}=0$.

$(\beta)$ : The second term converges strongly to 0 in $L^{2}(\Omega)$, because of the following estimates:

$$
\begin{gathered}
E\left[(i i)_{n}^{2}\right] \leq\|b\|_{\infty}^{2} E\left[\left(\int_{0}^{t} \int_{\mathbb{R}^{d}}\left|D u_{0}\left(\phi_{n, s}^{-1}(x)\right)-D u_{0}\left(\phi_{s}^{-1}(x)\right)\right|\left|D \phi_{n, s}^{-1}(x) \| \theta(x)\right| d x d s\right)^{2}\right. \\
\leq\|b\|_{\infty}^{2} t\|\theta\|_{L^{1}\left(\mathbb{R}^{d}\right)} \int_{0}^{t} \int_{\mathbb{R}^{d}} E\left[\left|D u_{0}\left(\phi_{n, s}^{-1}(x)\right)-D u_{0}\left(\phi_{s}^{-1}(x)\right)\right|^{2}\left|D \phi_{n, s}^{-1}(x)\right|^{2}\right]|\theta(x)| d x d s \\
\leq\|b\|_{\infty}^{2} t\|\theta\|_{L^{1}\left(\mathbb{R}^{d}\right)} \int_{0}^{t} \int_{\mathbb{R}^{d}}\left(E\left[\left|D u_{0}\left(\phi_{n, s}^{-1}(x)\right)-D u_{0}\left(\phi_{s}^{-1}(x)\right)\right|^{4}\right]\right)^{1 / 2}\left(E\left[\left|D \phi_{n, s}^{-1}(x)\right|^{4}\right]\right)^{1 / 2}|\theta(x)| d x d s \\
\leq\|b\|_{\infty}^{2} t\|\theta\|_{L^{1}\left(\mathbb{R}^{d}\right)} \sup _{k, r, y}\left(E\left[\left|D \phi_{k, r}^{-1}(y)\right|^{4}\right]\right)^{1 / 2} \\
\times \int_{0}^{t} \int_{\mathbb{R}^{d}}\left(E\left[\left|D u_{0}\left(\phi_{n, s}^{-1}(x)\right)-D u_{0}\left(\phi_{s}^{-1}(x)\right)\right|^{4}\right]\right)^{1 / 2}|\theta(x)| d x d s .
\end{gathered}
$$

The above estimates are consequences of Hölder's inequality. Since $D u_{0}$ is bounded and continuous, the right hand side of the above inequality converges to 0 by dominated convergence.

$(\gamma)$ : For the last term, let $X \in L^{2}(\Omega)$ and consider

$$
E\left[(i i i)_{n} X\right]=\int_{0}^{t} E\left[\int_{\mathbb{R}^{d}} D u_{0}\left(\phi_{s}^{-1}(x)\right)\left(D \phi_{n, s}^{-1}(x)-D \phi_{s}^{-1}(x)\right) \cdot b(s, x) \theta(x) X d x\right] d s
$$

Now, for each $s$, since $D u_{0}, b$ and $\theta$ are bounded and $D \phi_{s}^{-1}$ is the weak limit of $D \phi_{n, s}^{-1}$, this expression tends to 0 as $n \rightarrow \infty$.

\section{Uniqueness of weak solutions:}

Let us assume that $u$ is a weak solution to the stochastic transport equation (3.28) with $\sup _{0 \leq s \leq 1, x \in \mathbb{R}^{d}} E\left[|D u(s, x)|^{4}\right]<\infty$. We will show that

$$
u(t, x)=u_{0}\left(\phi_{t}^{-1}(x)\right. \text { a.e. }
$$

This will guarantee uniqueness of the solution to the transport equation. So let $V$ be a bounded and open subset of $\mathbb{R}^{d}$ and consider for the locally integrable function $u(t, \cdot)$ on $\mathbb{R}^{d}$ its mollification

$$
u_{\epsilon}(t, x)=\left(u * \eta_{\epsilon}\right)=\int_{\mathbb{R}^{d}} u(t, y) \eta_{\epsilon}(x-y) d y,
$$

with respect to the standard mollifier $\eta$.

We observe that $u_{\epsilon}$ satisfies the equation

$$
u_{\epsilon}(t, x)=u_{0, \epsilon}(x)-\int_{0}^{t}(b \cdot D u)_{\epsilon}(s, x) d s-\int_{0}^{t}(D u)_{\epsilon}(s, x) \circ d B_{s} .
$$


Then using the Itô-Ventzell formula applied to $u_{\epsilon}$ and $\phi_{t}(x)$ (see [22]) gives

$$
u_{\epsilon}\left(t, \phi_{t}(x)\right)=u_{0, \epsilon}(x)+\int_{0}^{t}\left((D u)_{\epsilon}\left(s, \phi_{s}(x)\right) \cdot b\left(s, \phi_{s}(x)\right)-(b \cdot D u)_{\epsilon}\left(s, \phi_{s}(x)\right)\right) d s .
$$

Now let $\tau \in L^{\infty}(\Omega)$ and $\theta$ be a smooth function with compact support in $V$. Then it follows from (3.30) that

$$
\begin{aligned}
& E\left[\tau \int_{V} \theta(x) u_{\epsilon}\left(t, \phi_{t}(x)\right) d x\right] \\
= & E\left[\tau \int_{V} \theta(x) u_{0, \epsilon}(x) d x\right] \\
+ & E\left[\tau \int_{0}^{t} \int_{V} \theta(x)\left((D u)_{\epsilon}\left(s, \phi_{s}(x)\right) \cdot b\left(s, \phi_{s}(x)\right)-(b \cdot D u)_{\epsilon}\left(s, \phi_{s}(x)\right)\right) d x d s\right] .
\end{aligned}
$$

Using Theorem 3.22 applied to $\phi_{t}^{-1}(\cdot)$ we obtain

$$
\begin{aligned}
& E\left[\tau \int_{0}^{t} \int_{V} \theta(x)\left((D u)_{\epsilon}\left(s, \phi_{s}(x)\right) \cdot b\left(s, \phi_{s}(x)\right)-(b \cdot D u)_{\epsilon}\left(s, \phi_{s}(x)\right)\right) d x d s\right] \\
= & E\left[\tau \int_{0}^{t} \int_{\mathbb{R}^{d}} \chi_{\phi_{s}(V)}(x) \theta\left(\phi_{s}^{-1}(x)\right)\left((D u)_{\epsilon}(s, x) \cdot b(s, x)-(b \cdot D u)_{\epsilon}(s, x)\right)\left|\operatorname{det}\left(J \phi_{s}^{-1}(x)\right)\right| d x d s\right] \\
= & I_{1}+I_{2},
\end{aligned}
$$

where

$$
I_{1}:=E\left[\tau \int_{0}^{t} \int_{\mathbb{R}^{d}} \chi_{\phi_{s}(V)}(x) \theta\left(\phi_{s}^{-1}(x)\right)\left((D u)_{\epsilon}(s, x) \cdot b(s, x)\right)\left|\operatorname{det}\left(J \phi_{s}^{-1}(x)\right)\right| d x d s\right]
$$

and

$$
I_{2}:=-E\left[\tau \int_{0}^{t} \int_{\mathbb{R}^{d}} \chi_{\phi_{s}(V)}(x) \theta\left(\phi_{s}^{-1}(x)\right)(b \cdot D u)_{\epsilon}(s, x)\left|\operatorname{det}\left(J \phi_{s}^{-1}(x)\right)\right| d x d s\right] .
$$

Since $V$ is bounded, there exists a $n \in \mathbb{N}$ such that $V \subset \bar{V} \subset W:=(-n, n)^{d}$. Then we get

$$
\begin{aligned}
\left\|(D u)_{\epsilon}\right\|_{L^{2}\left(\phi_{s}(V)\right)} & \leq\|D u\|_{L^{2}\left(\phi_{s}(W)\right)} \\
\left\|(b \cdot D u)_{\epsilon}\right\|_{L^{2}\left(\phi_{s}(V)\right)} & \leq\|b \cdot D u\|_{L^{2}\left(\phi_{s}(W)\right)} \\
& \leq\|b\|_{\infty}\|D u\|_{L^{2}\left(\phi_{s}(W)\right)} .
\end{aligned}
$$


Using (3.36), Hölder's inequality, Fubini's theorem and Theorem 3.22, we obtain

$$
\begin{aligned}
I_{1} \leq & C E\left[\int_{0}^{t}\left(\int_{\mathbb{R}^{d}}\left(\chi_{\phi_{s}(V)}(x) \theta\left(\phi_{s}^{-1}(x)\right) b(s, x)\left|\operatorname{det}\left(J \phi_{s}^{-1}(x)\right)\right|\right)^{2} d x\right)^{\frac{1}{2}}\right. \\
& \left.\cdot\left(\int_{\mathbb{R}^{d}} \chi_{\phi_{s}(W)}(x)|D u(s, x)|^{2} d x\right)^{\frac{1}{2}} d s\right] \\
\leq & C \int_{0}^{t} E\left[\int_{\mathbb{R}^{d}}\left(\chi_{\phi_{s}(V)}(x) \theta\left(\phi_{s}^{-1}(x)\right) b(s, x)\left|\operatorname{det}\left(J \phi_{s}^{-1}(x)\right)\right|\right)^{2} d x\right]^{\frac{1}{2}} \\
& \cdot E\left[\int_{\mathbb{R}^{d}} \chi_{\phi_{s}(W)}(x)|D u(s, x)|^{2} d x\right]^{\frac{1}{2}} d s \\
\leq & C \int_{0}^{t} E\left[\int_{\mathbb{R}^{d}} \chi_{\phi_{s}(V)}(x)\left|\operatorname{det}\left(J \phi_{s}^{-1}(x)\right)\right|^{2} d x\right]^{\frac{1}{2}} \\
& \cdot E\left[\int_{\mathbb{R}^{d}} \chi_{\phi_{s}(W)}(x)|D u(s, x)|^{2} d x\right]^{\frac{1}{2}} d s \\
\leq & C \int_{0}^{t}\left(\int_{\mathbb{R}^{d}} E\left[\chi_{\phi_{s}(V)}(x)\right]^{\frac{1}{2}} E\left[\left|\operatorname{det}\left(J \phi_{s}^{-1}(x)\right)\right|^{4}\right]^{\frac{1}{2}} d x\right)^{\frac{1}{2}} \\
& \cdot\left(\int_{\mathbb{R}^{d}} E\left[\chi_{\phi_{s}(W)}(x)\right]^{\frac{1}{2}} E\left[|D u(s, x)|^{4}\right]^{\frac{1}{2}} d x\right)^{\frac{1}{2}} d s \\
\leq & C \sup _{0 \leq s \leq 1, x \in \mathbb{R}^{d}} E\left[\left|\operatorname{det}\left(J \phi_{s}^{-1}(x)\right)\right|^{4}\right]^{\frac{1}{2}} \sup _{0 \leq s \leq 1, x \in \mathbb{R}^{d}} E\left[|D u(s, x)|^{4}\right]^{\frac{1}{2}} \\
& \cdot \int_{0}^{t}\left(\int_{\mathbb{R}^{d}} E\left[\chi_{\phi_{s}(V)}(x)\right]^{\frac{1}{2}} d x\right) d s \\
\leq & C \int_{0}^{t}\left(\int_{\mathbb{R}^{d}} E\left[\chi_{\phi_{s}(V)}(x)\right]^{\frac{1}{2}} d x\right) d s
\end{aligned}
$$

for a constant $C$ depending on the sizes of $V, \theta$ and $b$, since

$$
\sup _{0 \leq s \leq 1, x \in \mathbb{R}^{d}} E\left[\left|\operatorname{det}\left(J \phi_{s}^{-1}(x)\right)\right|^{4}\right] \leq M<\infty
$$

because of Lemma 3.7 applied to $\phi_{s}^{-1}(x)$.

Further, it follows from Girsanov's theorem, Hölder's inequality and the symmetry of the distribution of the Brownian motion that

$$
\begin{aligned}
& \int_{0}^{t} \int_{\mathbb{R}^{d}} E\left[\chi_{\phi_{s}(W)}(x)\right]^{\frac{1}{2}} d x d s \\
= & \int_{0}^{t} \int_{\mathbb{R}^{d}}\left(\mu\left(\phi_{s}^{-1}(x) \in W\right)\right)^{\frac{1}{2}} d x d s \\
\leq & C \int_{0}^{t} \int_{\mathbb{R}^{d}}\left(\mu\left(B_{s}+x \in W\right)\right)^{\frac{1}{4}} d x d s \\
= & C \int_{0}^{t} \int_{\mathbb{R}^{d}}\left(\mu\left(B_{s}+x \in(-n, n)^{d}\right)^{\frac{1}{4}} d x d s\right. \\
\leq & C \int_{0}^{t}\left(2 \int_{0}^{\infty}\left(1-\Phi\left(\frac{-n+y}{\sqrt{s}}\right)\right)^{\frac{1}{4}} d y\right)^{d} d s,
\end{aligned}
$$

where $\Phi$ is the standard normal distribution function. 
On the other hand we know that

$$
1-\Phi(x) \leq \frac{1}{2 \pi x} \exp \left(-x^{2} / 2\right)
$$

for all $x>0$ (see [3]).

So

$$
\begin{aligned}
& \int_{0}^{t} \int_{\mathbb{R}^{d}} E\left[\chi_{\phi_{s}(W)}(x)\right]^{\frac{1}{2}} d x d s \\
\leq & C \int_{0}^{t}\left(2 \int_{0}^{n}\left(1-\Phi\left(\frac{-n+y}{\sqrt{s}}\right)\right)^{\frac{1}{4}} d y+2 \int_{n}^{\infty}\left(1-\Phi\left(\frac{-n+y}{\sqrt{s}}\right)\right)^{\frac{1}{4}} d y\right)^{d} d s \\
\leq & K \int_{0}^{t}\left(\left(\int_{0}^{n}\left(1-\Phi\left(\frac{-n+y}{\sqrt{s}}\right)\right)^{\frac{1}{4}} d y\right)^{d}+\left(\int_{n}^{\infty}\left(1-\Phi\left(\frac{-n+y}{\sqrt{s}}\right)\right)^{\frac{1}{4}} d y\right)^{d}\right) d s \\
\leq & \left.M\left(1+\int_{0}^{t}\left(\int_{n}^{\infty}\left(\frac{\sqrt{s}}{2 \pi(y-n)} \exp \left(-(y-n)^{2} / 2 s\right)\right)^{\frac{1}{4}} d y\right)^{d}\right) d s\right) \\
= & \left.M\left(1+\int_{0}^{t}\left(\int_{0}^{\infty}\left(\frac{\sqrt{s}}{2 \pi y} \exp \left(-y^{2} / 2 s\right)\right)^{\frac{1}{4}} d y\right)^{d}\right) d s\right) \\
= & \left.M\left(1+\int_{0}^{t}\left(\int_{0}^{\infty} \sqrt{s}\left(\frac{1}{2 \pi y} \exp \left(-y^{2} / 2\right)\right)^{\frac{1}{4}} d y\right)^{d}\right) d s\right) \\
\leq & L<\infty .
\end{aligned}
$$

Furthermore, since

$$
(D u)_{\epsilon} \longrightarrow D u \text { in } L_{l o c}^{p}\left(\mathbb{R}^{d}\right)
$$

for all $p>1$ and since

$$
\int_{\mathbb{R}^{d}}\left(\chi_{\phi_{s}(V)}(x) \theta\left(\phi_{s}^{-1}(x)\right) b(s, x)\left|\operatorname{det}\left(J \phi_{s}^{-1}(x)\right)\right|\right)^{2} d x<\infty \text { a.e. }
$$

because of the above estimates, we obtain

$$
\begin{aligned}
& \int_{\mathbb{R}^{d}} \chi_{\phi_{s}(V)}(x) \theta\left(\phi_{s}^{-1}(x)\right)\left((D u)_{\epsilon}(s, x) \cdot b(s, x)\right)\left|\operatorname{det}\left(J \phi_{s}^{-1}(x)\right)\right| d x \\
\longrightarrow & \int_{\mathbb{R}^{d}} \chi_{\phi_{s}(V)}(x) \theta\left(\phi_{s}^{-1}(x)\right)((D u)(s, x) \cdot b(s, x))\left|\operatorname{det}\left(J \phi_{s}^{-1}(x)\right)\right| d x
\end{aligned}
$$

for $\epsilon \searrow 0 \mu \times d s-$ a.e.

On the other hand the latter expression w.r.t. $\epsilon$ is dominated by the integrable term

$$
\left(\int_{\mathbb{R}^{d}}\left(\chi_{\phi_{s}(V)}(x) \theta\left(\phi_{s}^{-1}(x)\right) b(s, x)\left|\operatorname{det}\left(J \phi_{s}^{-1}(x)\right)\right|\right)^{2} d x\right)^{\frac{1}{2}}\left(\int_{\mathbb{R}^{d}} \chi_{\phi_{s}(W)}(x)|D u(s, x)|^{2} d x\right)^{\frac{1}{2}} .
$$

So using dominated convergence it follows from (3.37) and (3.39) that

$$
\begin{aligned}
I_{1}= & I_{1}(\epsilon) \longrightarrow \\
& E\left[\tau \int_{0}^{t} \int_{\mathbb{R}^{d}} \chi_{\phi_{s}(V)}(x) \theta\left(\phi_{s}^{-1}(x)\right)((D u)(s, x) \cdot b(s, x))\left|\operatorname{det}\left(J \phi_{s}^{-1}(x)\right)\right| d x d s\right]
\end{aligned}
$$

for $\epsilon \searrow 0$. 
Similarly to $I_{1}$ we also get

$$
\begin{aligned}
I_{2}= & I_{2}(\epsilon) \longrightarrow \\
& -E\left[\tau \int_{0}^{t} \int_{\mathbb{R}^{d}} \chi_{\phi_{s}(V)}(x) \theta\left(\phi_{s}^{-1}(x)\right)(b \cdot D u)(s, x)\left|\operatorname{det}\left(J \phi_{s}^{-1}(x)\right)\right| d x d s\right]
\end{aligned}
$$

for $\epsilon \searrow 0$

and

$$
E\left[\tau \int_{V} \theta(x) u_{\epsilon}\left(t, \phi_{t}(x)\right) d x\right] \longrightarrow E\left[\tau \int_{V} \theta(x) u\left(t, \phi_{t}(x)\right) d x\right]
$$

as $\epsilon \searrow 0$.

In addition, because of the assumptions on $u_{0}$ it is clear that

$$
E\left[\tau \int_{V} \theta(x) u_{0, \epsilon}(x) d x\right] \longrightarrow E\left[\tau \int_{V} \theta(x) u_{0}(x) d x\right]
$$

as $\epsilon \searrow 0$.

Altogether we can conclude that

$$
E\left[\tau \int_{\mathbb{R}^{d}} \theta(x) u\left(t, \phi_{t}(x)\right) d x=E\left[\tau \int_{\mathbb{R}^{d}} \theta(x) u_{0}(x) d x\right]\right.
$$

for all $\tau \in L^{\infty}(\Omega)$ and compactly supported smooth functions $\theta$. Hence

$$
u\left(t, \phi_{t}(x)\right)=u_{0}(x)
$$

$\mu \times d x-$ a.e.

Since $\phi_{t}^{-1}(\cdot)$ satisfies the Lusin condition in Theorem 3.22 on bounded open subsets we can find a $\Omega^{*}$ with $\mu\left(\Omega^{*}\right)=1$ such that for all $\omega \in \Omega^{*}$

$$
u(t, x)=u_{0}\left(\phi_{t}^{-1}(x)\right) d x-\text { a.e. }
$$

Due to the continuity of $u$ with respect to time the latter relation also holds uniformly in $t$.

Finally, the Malliavin differentiability of (a version) of $u(t, x)$ is a consequence of the fact that $\phi_{t}^{-1}(x)$ is Malliavin differentiable (see [26]) and of the chain rule for Malliavin derivatives (see [31]).

\subsection{Application to ODE's}

In this section we employ the approach developed in Section 3.2 to study the existence of absolutely continuous solutions $x \longmapsto X_{t}^{x}$ of the time-homogeneous (deterministic) ODE

$$
d X_{t}^{x}=b\left(X_{t}^{x}\right) d t, X_{0}=x \in \mathbb{R}, t \in \mathbb{R},
$$

where $b: \mathbb{R} \longrightarrow \mathbb{R}$ is a discontinuous function. More precisely, we show that the sequence of solutions $X^{n, x}, n \geq 1$ to the perturbed equation

$$
d X_{t}^{n, x}=b\left(X_{t}^{n, x}\right) d t+\frac{1}{n} d B_{t}, X_{0}^{n, x}=x \in \mathbb{R}, t \in \mathbb{R},
$$

converge to a solution $X^{n, x}$ of the ODE (3.43). Furthermore, we show that this family of solutions to the ODE is absolutely continuous in $x \in \mathbb{R}$.

We begin with the following observation: 
Proposition 3.24 Let $b=\left(b_{1}, \ldots, b_{d}\right):[0,1] \times \mathbb{R}^{d} \longrightarrow \mathbb{R}^{d}$ be a bounded Borel measurable function with at most countably many points of discontinuities in the space variable uniformly in time. Further, suppose that there exist constants $m_{i}>0, i=1, \ldots, d$ such that for each $i$

$$
\text { either } m_{i} \leq b_{i}(t, y) \text { for all } t, y \text { or } b_{i}(t, y) \leq-m_{i} \text { for all } t, y \text {. }
$$

Then there exists for all initial values $x \in \mathbb{R}^{d}$ a solution to the $O D E$

$$
d X_{t}^{x}=b\left(t, X_{t}^{x}\right) d t, X_{0}=x, 0 \leq t \leq 1 .
$$

Proof. By a result of A. Y. Veretennikov [39] we know that the perturbed equation

$$
d X_{t}^{n, x}=b\left(t, X_{t}^{n, x}\right) d t+\frac{1}{n} d B_{t}, X_{0}^{n, x}=x \in \mathbb{R}^{d}, 0 \leq t \leq 1
$$

has a unique strong solution $X^{n, x}$ with continuous paths for all $n \geq 1$.

On the other hand, we also know that the Brownian paths are $\alpha$-Hölder continuous a.e. for all $\alpha<\frac{1}{2}$ See e.g. [20]. Let us fix a $\omega$ in some $\Omega^{*}$ with $\mu\left(\Omega^{*}\right)=1$ on which all those solutions and Brownian paths are concentrated. Then there exists a constant $C=C(\omega)<\infty$ such that for all $0 \leq t_{1}, t_{2} \leq 1$ and $n \geq 1$

$$
\begin{aligned}
\left|X_{t_{1}}^{n, x}-X_{t_{2}}^{n, x}\right| & \leq M\left|t_{1}-t_{2}\right|+\frac{C}{n}\left|t_{1}-t_{2}\right|^{\alpha} \\
& \leq M\left|t_{1}-t_{2}\right|+C\left|t_{1}-t_{2}\right|^{\alpha}
\end{aligned}
$$

for a constant $M<\infty$. Clearly, we also have

$$
\sup _{0 \leq t \leq 1}\left|X_{t}^{n, x}\right| \leq M<\infty
$$

uniformly in $n$ for some $M$. So it follows from the theorem of Arzela-Ascoli that

$$
X_{.}^{n_{k}, x} \underset{k \longrightarrow \infty}{\longrightarrow} X^{x}=\left(X_{.}^{x,(1)}, \ldots, X_{.}^{x,(d)}\right) \text { in } C\left([0,1] ; \mathbb{R}^{d}\right)
$$

for some subsequence $\left\{n_{k}\right\}_{k=1}^{\infty}$ of $\{n\}_{n=1}^{\infty}$. Thus

$$
X_{t}^{x,(j)}=x_{j}+\lim _{n \longrightarrow \infty} \int_{0}^{t} b_{j}\left(s, X_{s}^{n_{k}, x}\right) d s
$$

for all $t \in[0,1], j \in\{1,2, \cdots, d\}$. Thus for each $1 \leq j \leq d$, either

$$
X_{t_{1}}^{x,(j)}-X_{t_{2}}^{x,(j)}=\lim _{n \longrightarrow \infty} \int_{t_{1}}^{t_{2}} b_{j}\left(s, X_{s}^{n_{k}, x}\right) d s \geq m_{j}>0, t_{1}<t_{2}
$$

or

$$
X_{t_{1}}^{x,(j)}-X_{t_{2}}^{x,(j)}=\lim _{n \longrightarrow \infty} \int_{t_{1}}^{t_{2}} b_{j}\left(s, X_{s}^{n_{k}, x}\right) d s \leq-m_{j}<0, t_{1}<t_{2} .
$$

So any of the components $X^{x,(j)}$ of $X^{x}$ is a bijection on $[0,1]$. Hence $X_{t}^{x,(j)}$ can only hit the $j$-th projection of the points of discontinuities of $b$ in the space variable at most countably many times for $t \in[0,1]$. Therefore $X_{t}^{x}$ doesn't hit the discontinuity points of $b t$-a.e. Finally, using dominated convergence we get

$$
X_{t}^{x}=x+\int_{0}^{t} b\left(s, X_{s}^{x}\right) d s, 0 \leq t \leq 1 .
$$


Remark 3.25 In [38] it is shown that even if $b:[0, \infty) \longrightarrow[a, b]$ with $a>0$ is Borel measurable, then the ODE (3.43) has a solution in $[0, \infty)$.

In the sequel let us denote by

$$
\int_{0}^{t} \int_{\mathbb{R}} f(s, y) L^{X^{x}}(d y, d s)
$$

the integral of a bounded measurable function $f:[0,1] \times \mathbb{R} \longrightarrow \mathbb{R}$ with respect to the local time $L^{X^{x}}(d y, d s)$ of $X^{x}:=X^{0, x}$ (in time and space). For more information about local time-space integration the reader is referred to [3] or [37].

We also need the following auxiliary result:

Lemma 3.26 Let $b: \mathbb{R} \longrightarrow \mathbb{R}$ be a bounded Borel measurable function and let $\left(b_{n}\right)_{n \geq 1}$ in $C_{0}^{\infty}(\mathbb{R})$ be a sequence of functions such that

$$
b_{n}(y) \underset{n \longrightarrow \infty}{\longrightarrow} b(y) \text { a.e. }
$$

and

$$
\left|b_{n}(y)\right| \leq C
$$

for all $n \geq 1, y \in \mathbb{R}$ and some finite positive constant $C$. Denote by $X^{n, x}$ the unique strong solution to

$$
d X_{t}^{n, x}=b_{n}\left(X_{t}^{n, x}\right) d t+\delta d B_{t}, X_{0}^{n, x}=x \in \mathbb{R}, 0 \leq t \leq 1,
$$

where $\delta>0$ is a constant. Let $b_{n}^{\prime}$ be the derivative of $b_{n}$ for each $n \geq 1$.

Then the following convergence

$$
\exp \left(\int_{0}^{t} b_{n}^{\prime}\left(X_{s}^{n, x}\right) d s\right) \underset{n \longrightarrow \infty}{\longrightarrow} \exp \left(-\frac{1}{\delta^{2}} \int_{0}^{t} \int_{\mathbb{R}} b(y) L^{X^{x}}(d y, d s)\right)
$$

holds weakly in $L^{2}(\mathcal{U} \times[0,1] \times \Omega, d x \times d t \times d \mu)$ for all bounded open sets $\mathcal{U} \subset \mathbb{R}$.

\section{Proof.}

We start by noting that the set of functions

$$
\left(\varphi \otimes \exp \left\{\int_{0}^{1} h(s) d B_{s}\right\}\right)
$$

is total in $L^{2}(\mathcal{U} \times[0,1] \times \Omega, d x \times d t \times d \mu)$ when $\varphi$ ranges through $C_{0}^{\infty}(\mathcal{U} \times[0,1])$ and $h$ ranges through the step functions defined on $[0,1]$.

By Girsanov's theorem we have 


$$
\begin{aligned}
& \mid\left(\varphi \otimes \exp \left\{\int_{0}^{1} h(s) d B_{s}\right\}, \exp \left(\int_{0}^{t} b_{n}^{\prime}\left(X_{s}^{n, x}\right) d s\right)-\exp \left(-\frac{1}{\delta^{2}} \int_{0}^{t} \int_{\mathbb{R}} b(y) L^{X^{x}}(d y, d s)\right){ }_{L^{2}(\mathcal{U} \times[0,1] \times \Omega)} \mid\right. \\
= & \mid \int_{\mathbb{R}} \int_{0}^{1} \varphi(x, r) E\left[\exp \left\{\int_{0}^{1} h(s) d X_{s}^{n, x}\right\} \exp \left\{-\int_{0}^{t} b_{n}^{\prime}\left(x+B_{s}\right) d s\right\} \mathcal{E}\left(\int_{0}^{t} b_{n}\left(x+B_{u}\right) d B_{u}\right)\right] d r d x \\
- & \int_{\mathbb{R}} \int_{0}^{1} \varphi(x, r) E\left[\exp \left\{\int_{0}^{t} h(s) d X_{s}^{x}\right\} \exp \left\{-\frac{1}{\delta^{2}} \int_{0}^{t} \int_{\mathbb{R}} b(y) L^{\delta B \cdot+x}(d s, d y)\right\} \mathcal{E}\left(\int_{0}^{t} b\left(x+B_{u}\right) d B_{u}\right)\right] d r d x \mid \\
\leq & \mid \int_{\mathbb{R}} \int_{0}^{1} \varphi(x, r) E\left[\left(\exp \left\{\int_{0}^{1} h(s) d X_{s}^{n, x}\right\}-\exp \left\{\int_{0}^{1} h(s) d X_{s}^{x}\right\}\right)\right. \\
& \left.\times \exp \left\{\int_{0}^{t} b_{n}^{\prime}\left(x+B_{s}\right) d s\right\} \mathcal{E}\left(\int_{0}^{1} b_{n}\left(x+B_{u}\right) d B_{u}\right)\right] d r d x \mid \\
+ & \mid \int_{\mathbb{R}} \int_{0}^{1} \varphi(x, r) E\left[\exp \left\{\int_{0}^{1} h(s) d X_{s}^{x}\right\}\right. \\
& \left.\times\left(\exp \left\{\int_{0}^{t} b_{n}^{\prime}\left(x+B_{s}\right) d s\right\}-\exp \left\{-\frac{1}{\delta^{2}} \int_{0}^{t} \int_{\mathbb{R}} b(y) L^{\delta B .+x}(d s, d y)\right\}\right) \mathcal{E}\left(\int_{0}^{1} b_{n}\left(x+B_{u}\right) d B_{u}\right)\right] d r d x \mid \\
+ & \mid \int_{\mathbb{R}} \int_{0}^{1} \varphi(x, r) E\left[\exp \left\{\int_{0}^{1} h(s) d X_{s}^{x}\right\} \exp \left\{-\frac{1}{\delta^{2}} \int_{0}^{t} \int_{\mathbb{R}} b(y) L^{\delta B .+x}(d s, d y)\right\}\right. \\
& \left.\times\left(\mathcal{E}\left(\int_{0}^{1} b_{n}\left(x+B_{u}\right) d B_{u}\right)-\mathcal{E}\left(\int_{0}^{1} b\left(x+B_{u}\right) d B_{u}\right)\right)\right] d r d x \mid \\
= & \left.\left.: i)_{n}+i i\right)_{n}+i i i\right)_{n}
\end{aligned}
$$

For the first term, since

$$
\exp \left\{\int_{0}^{t} b_{n}^{\prime}\left(x+B_{s}\right) d s\right\}=1+\sum_{m \geq 1} \int_{0<s_{1}<\ldots s_{m}<t} \prod_{j=1}^{m} b_{n}^{\prime}\left(x+B_{s_{j}}\right) d s_{1} \ldots d s_{m},
$$

we get that the sequence

$$
\left\{\exp \left\{\int_{0}^{t} b_{n}^{\prime}\left(x+B_{s}\right) d s\right\} \mathcal{E}\left(\int_{0}^{1} b_{n}\left(x+B_{u}\right) d B_{u}\right)\right\}_{n \geq 1}
$$

is bounded in $L^{2}(\Omega)$ and we have 


$$
\begin{aligned}
i)_{n} \leq & \int_{0}^{1} \int_{\mathbb{R}}|\varphi(x, r)|\left\|\exp \left\{\int_{0}^{1} h(s) d X_{s}^{n, x}\right\}-\exp \left\{\int_{0}^{1} h(s) d X_{s}^{x}\right\}\right\|_{L^{2}(\Omega)} \times \\
& \left\|\exp \left\{\int_{0}^{t} b_{n}^{\prime}\left(x+B_{s}\right) d s\right\} \mathcal{E}\left(\int_{0}^{1} b_{n}\left(x+B_{u}\right) d B_{u}\right)\right\|_{L^{2}(\Omega)} d x d r .
\end{aligned}
$$

We know that $X_{t}^{n, x} \rightarrow X_{t}^{x}$ in $L^{2}(\Omega)$ and since $h$ is a step function we get by dominated convergence that

$$
\left.\lim _{n \rightarrow \infty} i\right)_{n}=0 .
$$

For the second term, by [3, Theorem 3.1] we have

$$
\int_{0}^{t} b_{n}^{\prime}\left(\delta B_{s}+x\right) d s=-\int_{0}^{t} \int_{\mathbb{R}} \frac{1}{\delta^{2}} b_{n}(y) L^{\delta B+x}(d s, d y)
$$

for all $t, \mu$-a.e.

On the other hand, we also know (see [37, p. 220]) that

$$
\begin{aligned}
& -\int_{0}^{t} \int_{\mathbb{R}} \frac{1}{\delta^{2}} b_{n}(y) L^{\delta B+x}(d s, d y) \\
= & 2\left(F_{n}\left(\delta B_{t}+x\right)-F_{n}(x)-\int_{0}^{t} \frac{1}{\delta^{2}} b_{n}\left(\delta B_{s}+x\right) \delta d B_{s}\right)
\end{aligned}
$$

where $F_{n}(y):=\int_{0}^{y} \frac{1}{\delta^{2}} b_{n}(u) d u$. The last expressions holds is true when $b_{n}$ is replaced by $b$. We see that the convergence

$$
\int_{0}^{t} b_{n}^{\prime}\left(\delta B_{s}+x\right) d s \rightarrow-\int_{0}^{t} \int_{\mathbb{R}} \frac{1}{\delta^{2}} b(y) L^{\delta B+x}(d s, d y)
$$

holds $\mu$ almost surely (possibly on a subequence). Similary as for $i)_{n}$ we may invoke dominated convergence to conclude

$$
\left.\lim _{n \rightarrow \infty} i i\right)_{n}=0 .
$$

For the last term notice that $\mathcal{E}\left(\int_{0}^{1} b_{n}\left(x+B_{u}\right) d B_{u}\right) \rightarrow \mathcal{E}\left(\int_{0}^{1} b\left(x+B_{u}\right) d B_{u}\right) \mu$-almost surely (possibly on a subsequence). Since $b_{n}$ is uniformly bounded we get that $\mathcal{E}\left(\int_{0}^{1} b_{n}\left(x+B_{u}\right) d B_{u}\right)-$ $\mathcal{E}\left(\int_{0}^{1} b\left(x+B_{u}\right) d B_{u}\right)$ is bounded in, say, $L^{4}(\Omega)$, and thus the same sequence is uniformly integrable when squared. We then get that

$$
\left\|\mathcal{E}\left(\int_{0}^{1} b_{n}\left(x+B_{u}\right) d B_{u}\right)-\mathcal{E}\left(\int_{0}^{1} b\left(x+B_{u}\right) d B_{u}\right)\right\|_{L^{2}(\Omega)} \rightarrow 0
$$

by the Vitali Convergence theorem. By dominated convergence we get

$$
\left.\lim _{n \rightarrow \infty} i i i\right)_{n}=0 .
$$


Theorem 3.27 Let $b: \mathbb{R} \longrightarrow \mathbb{R}$ be a bounded decreasing function such that either $m \leq b(y)$ for all $y$ or $b(y) \leq-m$ for all $y$ for some constant $m>0$. Then there exists a unique continuous function $(t, x) \longmapsto \widetilde{X}_{t}^{x}$ on $\mathbb{R} \times \mathbb{R}$ such that

$$
\widetilde{X}_{t}^{x}=x+\int_{0}^{t} b\left(\widetilde{X}_{s}^{x}\right) d s
$$

for all $t, x \in \mathbb{R}$. Moreover, the map

$$
(t, x) \longmapsto \widetilde{X}_{t}^{x}
$$

belongs to $L^{2}\left([0,1] ; W^{1,2}(\mathcal{U})\right)$ for any bounded open interval $\mathcal{U}$ in $\mathbb{R}$. The family

$$
\mathbb{R} \ni x \mapsto \widetilde{X}_{t}^{x} \in \mathbb{R}, t \in \mathbb{R},
$$

is a group of $W^{1,2}$ Sobolev diffeomorphisms on $\mathbb{R}$.

Proof. Uniqueness is easy. Indeed, suppose $Y_{t}^{x}$ is any solution of equation (3.46). Then

$$
\frac{d}{d t}\left(\widetilde{X}_{t}^{x}-Y_{t}^{x}\right)^{2}=2\left(\frac{d}{d t} \widetilde{X}_{t}^{x}-\frac{d}{d t} Y_{t}^{x}\right)\left(\widetilde{X}_{t}^{x}-Y_{t}^{x}\right)=2\left(b\left(\widetilde{X}_{t}^{x}\right)-b\left(Y_{t}^{x}\right)\right)\left(\widetilde{X}_{t}^{x}-Y_{t}^{x}\right) \leq 0
$$

since $b$ is decreasing. Integrating the above inequality from 0 to $t$, we get

$$
\left(\widetilde{X}_{t}^{x}-Y_{t}^{x}\right)^{2} \leq\left(\widetilde{X}_{0}^{x}-Y_{0}^{x}\right)^{2}=0
$$

This proves uniqueness.

We next prove existence of the flow for the ODE (3.46). It is sufficient to prove existence for $x \in \mathcal{U}$, an open bounded interval in $\mathbb{R}$ and for $t \in[0,1]$. Suppose $b_{m}, m \geq 1$ is a sequence of decreasing functions in $C_{0}^{\infty}(\mathbb{R})$ such that $b_{m}(y) \longrightarrow b(y)$ a.e. $m \longrightarrow \infty$ a.e. and $\left|b_{m}(y)\right| \leq C<\infty$ for all $m, y$ and for some positive constant $C$.

Consider the solution $X^{m, n, x}$ of the SDE

$$
X_{t}^{m, n, x}=x+\int_{0}^{t} b_{m}\left(X_{s}^{m, n, x}\right) d s+\frac{1}{n} B_{t}, 0 \leq t \leq 1
$$

for all $x \in \mathcal{U}, n, m \geq 1 \mu$-a.e.

We have (see [22])

$$
\frac{\partial}{\partial x} X_{t}^{m, n, x}=1+\int_{0}^{t} b_{m}^{\prime}\left(X_{s}^{m, n, x}\right) \frac{\partial}{\partial x} X_{s}^{m, n, x} d s, 0 \leq t \leq 1
$$

for all $x \in \mathcal{U}, n, m \geq 1 \mu$-a.e.

Therefore,

$$
\frac{\partial}{\partial x} X_{t}^{m, n, x}=\exp \left(\int_{0}^{t} b_{m}^{\prime}\left(X_{s}^{m, n, x}\right) d s\right) .
$$

Let $\varphi \in C_{0}^{\infty}(\mathcal{U}), \xi \in L^{\infty}(\Omega), h \in L^{\infty}([0,1])$.

Now from the proof of Lemma 3.15 in Section 3.2, it follows that for each $n \geq 1$ and $p>1$

$$
\sup _{0 \leq t \leq 1} \sup _{x \in \mathcal{U}} \sup _{m \geq 1} E\left[\left|\frac{\partial}{\partial x} X_{t}^{m, n, x}\right|^{p}\right] \leq M<\infty,
$$


where $M=M(n, p)$ is a positive constant.

The latter proof also shows that

$$
\begin{aligned}
& -\int_{\mathcal{U}} \int_{0}^{1} E\left[X_{t}^{m, n, x} \xi\right] h(t) \frac{\partial}{\partial x} \varphi(x) d t d x \\
& \underset{m \longrightarrow \infty}{\longrightarrow}-\int_{\mathcal{U}} \int_{0}^{1} E\left[X_{t}^{n, x} \xi\right] h(t) \frac{\partial}{\partial x} \varphi(x) d t d x .
\end{aligned}
$$

Since the map $(t, x) \longmapsto X_{t}^{x, n}$ belongs to $L^{2}\left([0,1] \times \Omega ; W^{1,2}(\mathcal{U})\right)$, then it has a continuous version $(t, x) \longmapsto \widetilde{X}_{t}^{n, x}$ which is absolutely continuous in $x$.

Using 3.47 and Lemma 3.26 we find

$$
\begin{aligned}
& -\int_{\mathcal{U}} \int_{0}^{1} E\left[X_{t}^{m, n, x} \xi\right] h(t) \frac{\partial}{\partial x} \varphi(x) d t d x \\
= & \int_{\mathcal{U}} \int_{0}^{1} E\left[\frac{\partial}{\partial x} X_{t}^{m, n, x} \xi\right] h(t) \varphi(x) d t d x \\
& \underset{m}{\longrightarrow} \int_{\mathcal{U}} \int_{0}^{1} E\left[\exp \left(-n^{2} \int_{0}^{t} \int_{\mathbb{R}} b(y) L^{X^{n, x}}(d s, d y)\right) \xi\right] h(t) \varphi(x) d t d x .
\end{aligned}
$$

Hence

$$
\frac{\partial}{\partial x} \widetilde{X}_{t}^{n, x}=\exp \left(-n^{2} \int_{0}^{t} \int_{\mathbb{R}} b(y) L^{X^{n, x}}(d s, d y)\right)
$$

for all $n \geq 1, d t \times d \mu \times d x$-a.e. So we may identify $\frac{\partial}{\partial x} \widetilde{X}_{t}^{n, x}$ with the process on the right hand side of (3.48). Then $(t, x) \longmapsto \widetilde{X}_{t}^{n, x}$ is continuous $\mu$-a.s.

Furthermore, since $b_{m}^{\prime}(y) \leq 0, y \in \mathbb{R}$ in (3.47) we can argue by weak convergence that the right hand side of (3.48) is dominated by a constant $K \geq 0$ uniformly in $n, x, t, \mu$-a.e.

Thus

$$
\sup _{0 \leq t \leq 1}\left|\widetilde{X}_{t}^{n, x_{1}}-\widetilde{X}_{t}^{n, x_{2}}\right| \leq K\left|x_{1}-x_{2}\right|
$$

for all $x_{1}, x_{2} \in \mathcal{U}, n \geq 1 \mu$-a.e.

On the other hand we may assume by Corollary 3.13 that $(t, x) \longmapsto X_{t}^{n, x}$ is continuous $\mu$-a.e. Hence we have

$$
\widetilde{X}_{t}^{n, x}=x+\int_{0}^{t} b\left(\widetilde{X}_{s}^{n, x}\right) d s+\frac{1}{n} B_{t}
$$

for all $n \geq 1, t, x, \mu-$ a.e.

So using the $\alpha$-Hölder continuity of Brownian paths, it follows that (for a fixed $\omega$ )

$$
\left|\widetilde{X}_{t_{1}}^{n, x}-\widetilde{X}_{t_{2}}^{n, x}\right| \leq M\left|t_{1}-t_{2}\right|+C(\omega)\left|t_{1}-t_{2}\right|^{\alpha}
$$

for all $0 \leq t_{1}, t_{2} \leq 1, n \geq 1$ and all $x \in \mathcal{U}$, where $\alpha<\frac{1}{2}, C(\omega)=C(\omega, \alpha)<\infty$ and $M<\infty$.

Let $\mathcal{V}$ be a compact sub-interval of $\mathcal{U}$. Fix an appropriate $\omega \in \Omega$. Then by the ArzelaAscoli theorem there is a subsequence $\left(n_{k}\right)$ such that $\left(x \longmapsto \widetilde{X}^{n_{k}, x}\right), k \geq 1$ converges in $C(\mathcal{V} ; C([0,1])$. Then repeated application of a weak compactness argument it follows that the limit, say $(t, x) \longmapsto \bar{X}_{t}^{x}$ belongs to $L^{2}\left([0,1] ; W^{1,2}(\mathcal{V})\right)\left(\mathcal{V}^{\mathcal{V}}\right.$ the interior of $\left.\mathcal{V}\right)$. Finally, and as in the proof of Proposition 3.24, it follows that $\bar{X}^{x}$. solves the ODE (3.46) for all $x$ in $\dot{\mathcal{V}}$. 
Remark 3.28 Using techniques of Malliavin calculus the authors in [25] prove that, for fixed $x \in \mathbb{R}$, the sequence $\left\{\widetilde{X}_{t}^{n, x}\right\}_{n=1}^{\infty}$ in the proof of Theorem 3.3 converges to $\widetilde{X}_{t}^{x}$ in $L^{2}(\mu)$ as $n \longrightarrow \infty$.

Curiously enough, the next theorem is a consequence of the deterministic result in Theorem 3.27 above. It establishes the existence of a perfect cocycle of $W_{l o c}^{1,2}$-Sobolev diffeomorphisms for solutions of the one-dimensional Stratonovich SDE:

$$
d X_{t}^{x}=b\left(X_{t}^{x}\right) \circ d W(t), t \in \mathbb{R}, X_{0}=x \in \mathbb{R},
$$

driven by a bounded decreasing diffusion coefficient $b: \mathbb{R} \rightarrow \mathbb{R}$ satisfying the conditions of Theorem 3.27.

Theorem 3.29 Let $b: \mathbb{R} \longrightarrow \mathbb{R}$ be a bounded decreasing function with a positive constant $m$ such that either $m \leq b(y)$ for all $y \in \mathbb{R}$ or $b(y) \leq-m$ for all $y \in \mathbb{R}$. Suppose $W: \mathbb{R} \times \Omega \rightarrow \mathbb{R}$ is one-dimensional Brownian motion such that $W(0)=0$, and $\theta: \mathbb{R} \times \Omega \rightarrow \Omega$ is the Wiener shift. Consider the Stratonovich SDE

$$
X_{t}^{x}=x+\int_{0}^{t} b\left(X_{s}^{x}\right) \circ d W(s), \quad t, x \in \mathbb{R} .
$$

Then the above SDE has a family of strong pathwise continuous solutions

$$
\mathbb{R} \times \mathbb{R} \ni(t, x) \longmapsto X_{t}^{x} \in \mathbb{R}
$$

such that $\left(X_{t}, \theta(t, \cdot)\right)$ is a perfect cocycle of $W_{\text {loc }}^{1,2}$-Sobolev diffeomorphisms on $\mathbb{R}$.

Proof. The idea of the proof is simple: We rescale the deterministic flow of the ODE (3.46) using the one-dimensional Brownian motion $W$. This is feasible by an appropriate application of Itô's formula due to Fölmer, Protter and Shiryayev [16].

To simplify notation, we denote by $Y(t, x):=\widetilde{X}_{t}^{x}, t, x \in \mathbb{R}$, the deterministic flow of the ODE (3.46). Define the random field:

$$
X_{t}^{x}:=Y(W(t), x), \quad t, x \in \mathbb{R} .
$$

We claim that the following equality

$$
\int_{0}^{W(t)} b(Y(u, x)) d u=\int_{0}^{t} b\left(X_{u}^{x}\right) \circ d W(u),, X_{0}=x \in \mathbb{R},
$$

holds for all $x \in \mathbb{R}$ a.s. for all $t \in \mathbb{R}$. To prove (3.52), we apply Itô's formula using the absolutely continuous change of variable $F(z):=\int_{0}^{z} b(Y(u, x)) d u, z \in \mathbb{R}$. Note that $F$ is locally of class $W^{1,2}$ because $F^{\prime}(z)=b(Y(z, x))$ for a.e. $z \in \mathbb{R}$. So by Itô's formula ([16]), it follows that

$$
\begin{aligned}
F(W(t)) & =\int_{0}^{t} F^{\prime}(W(u)) d W(u)+\frac{1}{2}\left[F^{\prime}(W), W\right](t) \\
& =\int_{0}^{t} F^{\prime}(W(u)) \circ d W(u) \\
& =\int_{0}^{t} b\left(X_{u}^{x}\right) \circ d W(u)
\end{aligned}
$$


a.s. for all $t \geq 0$. In the above relation, the bracket $[\cdot, \cdot]$ stands for the quadratic covariation. This proves our claim (3.52). Using (3.51), (3.46) and (3.52), we get

$$
\begin{aligned}
X_{t}^{x} & =Y(W(t), x) \\
& =x+\int_{0}^{W(t)} b(Y(u, x)) d u \\
& =x+\int_{0}^{t} b\left(X_{u}^{x}\right) \circ d W(u)
\end{aligned}
$$

a.s. for all $t \geq 0$. Hence $X_{t}^{x}, t, x \in \mathbb{R}$, is a family of solutions of the $\operatorname{SDE}$ (3.50). Since the map $[0,1] \ni t \mapsto Y(t, \cdot) \in W^{1,2}(U)$ belongs to $C\left([0,1], W^{1,2}(U)\right)$, then so do the maps $[0,1] \ni t \mapsto X_{\dot{t}}(\omega) \in W^{1,2}(U)$ for a.a. $\omega \in \Omega$, where $U$ is any bounded open interval in $\mathbb{R}$.

To prove the cocycle property for $\left(X_{t}, \theta(t, \cdot)\right)$, we use the group property for the ODE (3.46):

$$
Y\left(t_{1}, \cdot\right) \circ Y\left(t_{2}, \cdot\right)=Y\left(t_{1}+t_{2}, \cdot\right) \quad t_{1}, t_{2} \in \mathbb{R}
$$

Hence,

$$
\begin{aligned}
{\left[X_{t_{2}}\left(\theta\left(t_{1}, \omega\right)\right) \circ X_{t_{1}}(\omega)\right](x) } & =Y\left(\left(\theta\left(t_{1}, \omega\right)\left(t_{2}\right), Y\left(\omega\left(t_{1}\right), x\right)\right)\right. \\
& =Y\left(\omega\left(t_{1}+t_{2}\right), x\right) \\
& =X_{t_{1}+t_{2}}^{x}(\omega), \quad x, t_{1}, t_{2} \in \mathbb{R}, \omega \in \Omega .
\end{aligned}
$$

This completes the proof of the corollary.

Remark 3.30 It is rather remarkable that the Stratonovich SDE (3.50) admits the existence of a perfect cocycle of $W_{l o c}^{1,2}$-Sobolev diffeomorphisms with respect to a discontinuous diffusion coefficient. On the other hand, it is conceivable that the SDE (3.50) has more than one solution. In fact, we haven't even been able to find similar examples to (3.50) in the literature.

\subsection{Appendix}

The following result which is due to [4] provides a compactness criterion for subsets of $L^{2}\left(\mu ; \mathbb{R}^{d}\right)$ using Malliavin calculus. See e.g. [31], [13] or [7] for more information about Malliavin calculus.

Theorem 3.31 Let $\{(\Omega, \mathcal{A}, P) ; H\}$ be a Gaussian probability space, that is $(\Omega, \mathcal{A}, P)$ is a probability space and $H$ a separable closed subspace of Gaussian random variables of $L^{2}(\Omega)$, which generate the $\sigma$-field $\mathcal{A}$. Denote by $\mathbf{D}$ the derivative operator acting on elementary smooth random variables in the sense that

$$
\mathbf{D}\left(f\left(h_{1}, \ldots, h_{n}\right)\right)=\sum_{i=1}^{n} \partial_{i} f\left(h_{1}, \ldots, h_{n}\right) h_{i}, h_{i} \in H, f \in C_{b}^{\infty}\left(\mathbb{R}^{n}\right) .
$$

Further let $\mathbf{D}_{1,2}$ be the closure of the family of elementary smooth random variables with respect to the norm

$$
\|F\|_{1,2}:=\|F\|_{L^{2}(\Omega)}+\|\mathbf{D} F\|_{L^{2}(\Omega ; H)} .
$$

Assume that $C$ is a self-adjoint compact operator on $H$ with dense image. Then for any $c>0$ the set

$$
\mathcal{G}=\left\{G \in \mathbf{D}_{1,2}:\|G\|_{L^{2}(\Omega)}+\left\|C^{-1} \mathbf{D} G\right\|_{L^{2}(\Omega ; H)} \leq c\right\}
$$

is relatively compact in $L^{2}(\Omega)$. 
In order to formulate compactness criteria useful for our purposes, we need the following technical result which also can be found in [4].

Lemma 3.32 Let $v_{s}, s \geq 0$ be the Haar basis of $L^{2}([0,1])$. For any $0<\alpha<1 / 2$ define the operator $A_{\alpha}$ on $L^{2}([0,1])$ by

$$
A_{\alpha} v_{s}=2^{k \alpha} v_{s} \text {, if } s=2^{k}+j
$$

for $k \geq 0,0 \leq j \leq 2^{k}$ and

$$
A_{\alpha} 1=1 .
$$

Then for all $\beta$ with $\alpha<\beta<(1 / 2)$, there exists a constant $c_{1}$ such that

$$
\left\|A_{\alpha} f\right\| \leq c_{1}\left\{\|f\|_{L^{2}([0,1])}+\left(\int_{0}^{1} \int_{0}^{1} \frac{\left|f(t)-f\left(t^{\prime}\right)\right|^{2}}{\left|t-t^{\prime}\right|^{1+2 \beta}} d t d t^{\prime}\right)^{1 / 2}\right\} .
$$

A direct consequence of Theorem 3.31 and Lemma 3.32 is now the following compactness criterion which is essential for the proof of Lemma 3.14:

Corollary 3.33 Let $X_{n} \in \mathbb{D}_{1,2}, n=1,2 \ldots$, be a sequence of $\mathcal{F}_{1}$-measurable random variables such that there are constants $\alpha>0$ and $C>0$ with

$$
\begin{gathered}
\sup _{n} E\left[\left\|X_{n}\right\|^{2}\right] \leq C, \\
\sup _{n} E\left[\left\|D_{t} X_{n}-D_{t^{\prime}} X_{n}\right\|^{2}\right] \leq C\left|t-t^{\prime}\right|^{\alpha}
\end{gathered}
$$

for $0 \leq t^{\prime} \leq t \leq 1$ and

$$
\sup _{n} \sup _{0 \leq t \leq 1} E\left[\left\|D_{t} X_{n}\right\|^{2}\right] \leq C .
$$

where $D_{t}$ denotes Malliavin differentiation. Then the sequence $X_{n}, n=1,2 \ldots$, is relatively compact in $L^{2}(\Omega)$ ( $D_{t}$ the Malliavin derivative).

\section{Acknowledgement}

We wish to thank Erlend Storrøsten (CMA, University of Oslo) for valuable comments and fruitful discussions. 


\section{References}

[1] Ambrosio, L.: Transport equation and Cauchy problem for BV vector fields. Invent. Math. 158, 227-260 (2004).

[2] Attanasio, S.: Stochastic flows of diffeomorphisms for one-dimensional SDE with discontinuous drift. Elect. Comm. in Probab. 15, 213-226 (2010).

[3] Borodin, A. N., Salminen, P.: Handbook of Brownian Motion- Facts and Formulae. Birkhäuser, 2nd edition (2002).

[4] Da Prato, G., Malliavin, P., Nualart, D.: Compact families of Wiener functionals. C.R. Acad. Sci. Paris, t. 315, Série I, p. 1287-1291 (1992).

[5] Da Prato, G., Flandoli, F., Priola, E., Röckner, M.: Strong uniqueness for stochastic evolution equations in Hilbert spaces with bounded measurable drift. Preprint, arXiv:1109.0363 (2011).

[6] Davie, A. M.: Uniqueness of solutions of stochastic differential equations. Int. Math. Res. 24, Article ID rnm 124, (2007), 26 P.]

[7] Di Nunno, G., Øksendal, B., Proske, F.: Malliavin Calculus for Lévy Processes with Applications to Finance. Universitext, Springer (2009).

[8] Di Perna, R. J., Lions, P. L.: Ordinary differential equations, transport theory and Sobolev spaces. Invent. Math. 98, 511-547 (1989).

[9] Eisenbaum, N.: Integration with respect to local time. Potential Anal. 13, 303-328 (2000).

[10] Fedrizzi, E.: Uniqueness and flow theorems for solutions of SDE's with low regularity of the drift. Tesi di Laurea in Matematica, Università di Pisa (2009).

[11] Fedrizzi, E., Flandoli, F.: Pathwise uniqueness and continuous dependence for SDE's with non-regular drift. To appear in Stochastics (2010).

[12] Fedrizzi, E., Flandoli, F.: Hölder flow and differentiability of SDE's with non-regular drift. To appear in Stoch. Anal. Appl. (2010).

[13] Fedrizzi, E., Flandoli, F.: Noise prevents singularities in linear transport equations. Personal communication, work in progress, University of Pisa (2012).

[14] Flandoli, F., Gubinelli, E., Priola, E.: Well posedness of the transport equation by stochastic perturbation. Invent. Math. 180, 1-53 (2010).

[15] Flandoli, F.: Random Perturbation of PDE's and Fluid Dynamic Models. LNM 2015, Springer (2011).

[16] Föllmer, H., Protter, P., Shiryaev, A.N.: Quadratic covariation and an extension of Itô formula. Journal of Bernoulli Society, 1: 149169 (1995).

[17] Hajlasz, P.: Change of variables formula under minimal assumptions. Colloqium Mathematicum. Vol. LXIV, Fasc. 1, 93-101 (1993). 
[18] Heinonen, J., Kilpeläinen, Martio, O.: Nonlinear Potential Theory of Degenerate Elliptic Equations. Oxford Mathematical Monographs, Oxford University Press (1993).

[19] Hida, T., Kuo, H.-H., Potthoff, J., Streit, J.: White Noise. An Infinite Dimensional Approach. Kluwer (1993).

[20] Karatzas I., Shreve, S.E.:Brownian Motion and Stochastic Calculus, Springer-Verlag (1988).

[21] Kufner, A.: Weighted Sobolev Spaces. Wiley-Interscience (1985).

[22] Kunita, H.: Stochastic Flows and Stochastic Differential Equations. Cambridge University Press (1990).

[23] Krylov, N.V., Röckner, M.: Strong solutions of stochastic equations with singular time dependent drift. Prob. Theory Rel. Fields, Vol. 131, No. 2, 154-196 (2005).

[24] Malliavin , P.: Stochastic Analysis. Grundlehren der Mathematischen Wissenschaften, Vol. 313, Springer-Verlag, Berlin (1997).

[25] Menoukeu-Pamen, O., Meyer-Brandis, T., Proske, F.: A Gel'fand triple approach to the small noise problem for discontinuous ODE's. Preprint series, University of Oslo (2010).

[26] Menoukeu-Pamen, O., Meyer-Brandis, T., Nilssen, T., Proske, F., Zhang, T.: A variational approach to the construction and Malliavin differentiability of strong solutions of SDE's. To appear in Mathematische Annalen.

[27] Meyer-Brandis, T., Proske, F.:Construction of strong solutions of SDE's via Malliavin calculus. J. of Funct. Anal., 258, 3922-3953 (2010).

[28] Mohammed, S.E.A., Scheutzow, M.: Spatial estimates for stochastic flows in Euclidean space. The Annals of Probability, Vol. 26, No. 1, 56-77 (1998).

[29] Mohammed, S.E.A., Scheutzow, M.: The stable manifold theorem for non-linear stochastic systems with memory. I. Existence of the semiflow. J. of Funct. Anal. 205, 271-305 (2003).

[30] Mohammed, S.E.A., Scheutzow, M.: The stable manifold theorem for non-linear stochastic systems with memory II: The local stable manifold theorem. J. of Funct. Anal. 206, 253-306 (2004).

[31] Nualart, D.: The Malliavin Calculus and Related Topics. Springer (1995).

[32] Obata, N.: White Noise Caculus and Fock Space. LNM 1577, Berlin, Springer-Verlag (1994).

[33] Portenko, N. I.: Generalized Diffusion Processes. Transl. Math. Monographs, 83, Amer. Math. Soc., Providence, R. I. (1990).

[34] Protter, P.: Stochastic Integration and Differential Equations. A New Approach. Springer, 3rd edition (1995). 
[35] Reshetnyak, Y. G.: Some geometrical properties of functions and mappings with generalized derivatives. Sibirsk. Mat. Zh. 7 (4), 886-919 (in Russian) (1966).

[36] Reshetnyak, Y. G.: On the condition $N$ for mappings of class $W_{n, l o c}^{1}$, ibid. 28 (5), 149-153 (in Russian) (1987).

[37] Revuz, D., Yor, M.: Continuous Martingales and Brownian Motion. 3rd edition, Springer (2004).

[38] Rzymowski, W.: Existence of solutions for a class of discontinuous differential equations in $\mathbb{R}^{n}$. J. Math. Anal. Appl. 233, 634-643 (1999).

[39] Veretennikov, A.Y.: On the strong solutions of stochastic differential equations. Theory Probab. Appl., 24, 354-366 (1979).

[40] Ziemer, W.P.: Weakly Differentiable Functions. GTM, Springer (1989).

[41] Zvonkin, A.K.: A transformation of the state space of a diffusion process that removes the drift. Math.USSR (Sbornik), 22, 129-149 (1974). 


\title{
5 Malliavin differentiability and strong solutions for a class of SDE in Hilbert spaces
}

Franco Flandoli, Torstein Nilssen and Frank Proske

$$
\text { Preprint }
$$

\begin{abstract}
We consider a class of Hilbert-space valued SDE's where the drift coefficients are nonLipschitzian in the sense of Hölder-continuity. Using a novel technique based on Malliavin calculus we show in this paper the existence and uniqueness of a mild solution to such equations. We emphasize that our approach does not rely on the Yamada-Watanabe principle. Moreover our method gives the important additional insight that the obtained solution is Malliavin differentiable - a property which was recently shown to play a crucial role in the study of the geometry of certain optimal causal transference plans, [12].
\end{abstract}




\subsection{Introduction}

In a separable Hilbert space $H$, consider the stochastic differential equation

$$
d X_{t}=A X_{t} d t+B\left(t, X_{t}\right) d t+\sqrt{Q} d W_{t}, X_{0}=x
$$

where $A: D(A) \subset H \rightarrow H$ is the infinitesimal generator of a $C_{0}$-semigroup $e^{t A}, t \geq 0$; $B:[0, T] \times H \rightarrow H$ is continuous, $W$ is a cylindrical Wiener process and $Q$ is a non-negative selfadjoint bounded operator on $H$. If the operator $Q_{t}=\int_{0}^{t} e^{s A} Q e^{s A^{*}} d s$ is trace class, and suitable linear growth conditions on $B$ are assumed, weak existence is known for equation (5.1), see [5].

The aim of this paper is to prove Malliavin differentiablility and a direct proof of strong existence, under additional assumptions on $(A, B)$ stated in section 5.1.1. On $B$ we assume Hölder continuity in $x$ uniformly in $t$. On $A$ we assume certain non-degeneracy condition related to null-controllability. See [2] for the case of Hölder-coefficients. For merely bounded and measurable coefficients, see [9] and the recent work in [3].

\subsubsection{Notations and assumptions}

Norm and inner product in $H$ will be denoted $|\cdot|$ and $\langle\cdot, \cdot\rangle$. A complete orthonormal system $\left\{e_{n}\right\}_{n \geq 1}$ in $H$ is assumed to be fixed. If $\varphi: H \rightarrow H$, we shall denote its components with respect to $\left\{e_{n}\right\}_{n>1}$ by $\varphi_{n}: \varphi_{n}(x)=\left\langle\varphi(x), e_{n}\right\rangle$.

Given $\alpha, T>0$, we shall denote by $C\left([0, T] ; C_{b}^{\alpha}(H, H)\right)$ the space of all functions $G$ : $[0, T] \times H \rightarrow H$ which are continuous and bounded in $(t, x)$, and such that there exists $C>0$ such that

$$
|G(t, x)-G(t, y)| \leq C|x-y|^{\alpha}, x, y \in H, t \in[0, T] .
$$

We denote by $\|G\|_{\alpha, T}$ or simply $\|G\|_{\alpha}$ the norm

$$
\|G\|_{\alpha}=\sup _{t \in[0, T], x \in H}|G(t, x)|+\sup _{t \in[0, T]} \sup _{x \neq y \in H} \frac{|G(t, x)-G(t, y)|}{|x-y|^{\alpha}}
$$

We use the notation $\left\|G_{n}\right\|_{\alpha}$ also for the similar norm of the components $G_{n}(t, x)=\left\langle G(t, x), e_{n}\right\rangle$.

Let us now list the assumptions of this paper:

1. The operator $A$ is selfadjoint, with compact resolvent, and $A e_{n}=-\alpha_{n} e_{n}$, with nondecreasing positive $\left\{\alpha_{n}\right\}_{n \geq 1}$ such that

$$
\sum_{n=1}^{\infty} \frac{1}{\alpha_{n}^{1-\delta}}<\infty
$$

2. $B \in C\left([0, T] ; C_{b}^{\alpha}(H, H)\right)$ for some $\alpha, T>0$.

3.

$$
e^{t A}(H) \subset Q_{t}^{1 / 2}(H) \text { for all } t>0
$$

4. The well defined bounded operator $\Lambda_{t}=Q_{t}^{-1 / 2} e^{t A}$ satisfies

$$
\int_{0}^{T}\left\|\Lambda_{t}\right\|^{1+\theta} d t<\infty
$$

for some $\theta \geq \max (\alpha, 1-\alpha)$. 
Remark 5.1 From assumption (5.4) we have, in paricular,

$$
\int_{0}^{T}\left\|\Lambda_{t}\right\|^{1+\alpha} d t<\infty, \int_{0}^{T}\left\|\Lambda_{t}\right\|^{2-\alpha} d t<\infty
$$

\subsection{Idea of the method}

In this section we do not care about the rigor of the computations. The aim is to explain the idea.

For ever $n$, consider the following (backward) PDE in $H$ of Kolmogorov type, on some interval $[0, T]$ :

$$
\begin{aligned}
\frac{\partial U_{n}}{\partial t}+\frac{1}{2} \operatorname{Tr}\left(D^{2} U_{n} Q\right)+\left\langle A x, D U_{n}\right\rangle+\left\langle B, D U_{n}\right\rangle & =B_{n} \\
U_{n}(T, x) & =0
\end{aligned}
$$

Notice it is a non-homogeneous equation, opposite to the usual equations of Kolmogorov type; the right-hand-side $B_{n}$ is the $n$-component of $B$. If $U_{n}$ is a sufficiently regular solution, from Itô's formula we get

$$
\begin{aligned}
d U_{n}\left(t, X_{t}\right) & =B_{n}\left(t, X_{t}\right) d t+\left\langle D U_{n}\left(t, X_{t}\right), Q^{1 / 2} d W_{t}\right\rangle \\
& =\left\langle B\left(t, X_{t}\right) d t+D U\left(t, X_{t}\right) Q^{1 / 2} d W_{t}, e_{n}\right\rangle
\end{aligned}
$$

namely

$$
d U\left(t, X_{t}\right)=B\left(t, X_{t}\right) d t+D U\left(t, X_{t}\right) Q^{1 / 2} d W_{t}
$$

where $U(t, x)=\sum_{n} U_{n}(t, x) e_{n}$ and where we have used the PDE above. About our vectorvalued notations, let us stress that $U(t, \cdot): H \rightarrow H$, hence $D U\left(t, X_{t}\right) \in L(H, H)$. Moreover, for every $v \in H$,

$$
\left\langle D U\left(t, X_{t}\right) v, e_{n}\right\rangle=\left\langle D U_{n}\left(t, X_{t}\right), v\right\rangle .
$$

Formally speaking, the previous identity gives us a formula for $B\left(t, X_{t}\right) d t$ :

$$
B\left(t, X_{t}\right) d t=d U\left(t, X_{t}\right)-D U\left(t, X_{t}\right) Q^{1 / 2} d W_{t} .
$$

We put this formula in equation (5.1) and get

$$
d X_{t}=A X_{t} d t+d U\left(t, X_{t}\right)-D U\left(t, X_{t}\right) Q^{1 / 2} d W_{t}+Q^{1 / 2} d W_{t} .
$$

Now we follow the usual variation of constant method and get:

$$
\begin{aligned}
d_{s} e^{(t-s) A} X_{s} & =e^{(t-s) A} d U\left(s, X_{s}\right) \\
& +e^{(t-s) A} Q^{1 / 2} d W_{s}-e^{(t-s) A} D U\left(s, X_{s}\right) Q^{1 / 2} d W_{s}
\end{aligned}
$$


namely

$$
\begin{aligned}
X_{t}-e^{t A} x & =\int_{0}^{t} e^{(t-s) A} d U\left(s, X_{s}\right) \\
& +\int_{0}^{t} e^{(t-s) A} Q^{1 / 2} d W_{s}-\int_{0}^{t} e^{(t-s) A} D U\left(s, X_{s}\right) Q^{1 / 2} d W_{s} .
\end{aligned}
$$

Integrating by parts the first integral we finally get the equation

$$
\begin{gathered}
X_{t}=e^{t A}(x-U(0, x))+U\left(t, X_{t}\right)+\int_{0}^{t} A e^{(t-s) A} U\left(s, X_{s}\right) d s \\
+\int_{0}^{t} e^{(t-s) A} Q^{1 / 2} d W_{s}-I_{t}(X)
\end{gathered}
$$

where

$$
I_{t}(X):=\int_{0}^{t} e^{(t-s) A} D U\left(s, X_{s}\right) Q^{1 / 2} d W_{s} .
$$

The non-regular drift $B$ has been removed from the equation, this is the point of the trick. Several new terms appear, which however will be proved to have good Lipschitz properties.

In order to make rigorous this program we need: i) to solve the PDE (5.5) in a sufficiently regular space to be able to perform the previous computations (one bounded derivative plus an approximation argument is sufficient for this); ii) to prove that all the terms in equation (5.6) are Lipschitz continuous in the space variable (for this we need a uniform control of first and second derivatives). Morover, we need that the Lipschitz constant of the term $U\left(t, X_{t}\right)$ is small; we get this by taking small $T$ and using the condition $U_{n}(T, x)=0$.

\section{3 $H$-valued Ornstein-Uhlenbeck semigroup}

Let $R_{t}$ be the Ornstein-Uhlenbeck semigroup, defined on $B_{b}(H)$ as

$$
\begin{gathered}
R_{t} \varphi(x)=E\left[\varphi\left(Z_{t}^{x}\right)\right], \varphi \in B_{b}(H) \\
d Z_{t}^{x}=A Z_{t}^{x} d t+Q^{1 / 2} d W_{t}, Z_{0}^{x}=x .
\end{gathered}
$$

See [6], Chapter 6, for an extensive analysis of it. We introduce the analogous semigroup on $H$-valued functions:

$$
\mathcal{R}_{t} \Phi(x)=E\left[\Phi\left(Z_{t}^{x}\right)\right], \Phi \in B_{b}(H, H) .
$$

We have

$$
\left\langle\mathcal{R}_{t} \Phi(x), h\right\rangle=R_{t} \varphi_{h}(x), \varphi_{h}(x)=\langle\Phi(x), h\rangle, h \in H
$$

Theorem 5.2 Under the assumption (5.3), we have

$$
\Phi \in U C_{b}(H, H) \Rightarrow \mathcal{R}_{t} \Phi \in U C_{b}^{2}(H, H)
$$

for all $t>0$. The differential $D \mathcal{R}_{t} \Phi(x) \in L(H, H)$ at a given point $x \in H$ is the linear opeartor given by

$$
D \mathcal{R}_{t} \Phi(x) g=\int_{H}\left\langle\Lambda_{t} g, Q_{t}^{-1 / 2} y\right\rangle \Phi\left(e^{A t} x+y\right) N_{Q_{t}}(d y)
$$


or

$$
\begin{aligned}
\left\langle D \mathcal{R}_{t} \Phi(x) g, h\right\rangle & =\left\langle D R_{t} \phi_{h}(x), g\right\rangle \\
& =\int_{H}\left\langle\Lambda_{t} g, Q_{t}^{-1 / 2} y\right\rangle \phi_{h}\left(e^{A t} x+y\right) N_{Q_{t}}(d y)
\end{aligned}
$$

for all $t>0, g, h \in H$. The second derivative $D^{2} \mathcal{R}_{t} \Phi(x) \in L(H, L(H, H))$ at a given point $x \in H$, is given by (recall that $D^{2} \mathcal{R}_{t} \Phi(x)$ is a linear operator in $H$, for every $g \in H$ )

$$
\begin{gathered}
{\left[D^{2} \mathcal{R}_{t} \Phi(x) g\right] k} \\
=\int_{H}\left[\left\langle\Lambda_{t} g, Q_{t}^{-1 / 2} y\right\rangle\left\langle\Lambda_{t} k, Q_{t}^{-1 / 2} y\right\rangle-\left\langle\Lambda_{t} g, \Lambda_{t} k\right\rangle\right] \Phi\left(e^{t A} x+y\right) N_{Q_{t}}(d y)
\end{gathered}
$$

or

$$
\begin{gathered}
\left\langle\left[D^{2} \mathcal{R}_{t} \Phi(x) g\right] k, h\right\rangle=\left\langle D^{2} R_{t} \phi_{h}(x) g, k\right\rangle \\
=\int_{H}\left[\left\langle\Lambda_{t} g, Q_{t}^{-1 / 2} y\right\rangle\left\langle\Lambda_{t} k, Q_{t}^{-1 / 2} y\right\rangle-\left\langle\Lambda_{t} g, \Lambda_{t} k\right\rangle\right] \phi_{h}\left(e^{t A} x+y\right) N_{Q_{t}}(d y)
\end{gathered}
$$

for all $t>0, g, k, h \in H$. If $\Phi \in U C_{b}^{1}(H, H)$ then

$$
\left\langle\left[D^{2} \mathcal{R}_{t} \Phi(x) g\right] k, h\right\rangle=\int_{H}\left\langle\Lambda_{t} k, Q_{t}^{-1 / 2} y\right\rangle\left\langle D \phi_{h}\left(e^{t A} x+y\right), e^{t A} g\right\rangle N_{Q_{t}}(d y) .
$$

Finally,

$$
\begin{gathered}
\left\|D \mathcal{R}_{t} \Phi(x)\right\| \leq\left\|\Lambda_{t}\right\|\|\Phi\|_{0} \\
\left\|D^{2} \mathcal{R}_{t} \Phi(x)\right\| \leq \sqrt{2}\left\|\Lambda_{t}\right\|^{2}\|\Phi\|_{0} \\
\left\|D^{2} \mathcal{R}_{t} \Phi(x)\right\| \leq\left\|e^{t A}\right\|\left\|\Lambda_{t}\right\|\|\Phi\|_{1}
\end{gathered}
$$

Proof. Step 1. Let us chech that the right hand side of (5.8), namely the mapping

$$
g \mapsto I_{t, x} g:=\int_{H}\left\langle\Lambda_{t} g, Q_{t}^{-1 / 2} y\right\rangle \Phi\left(e^{A t} x+y\right) N_{Q_{t}}(d y)
$$

defines a linear bounded operator in $H ; x \in H$ and $t>0$ are given. The integral is a well defined element of $H$, because

$$
\begin{gathered}
\int_{H}\left|\left\langle\Lambda_{t} g, Q_{t}^{-1 / 2} y\right\rangle \Phi\left(e^{A t} x+y\right)\right|^{2} N_{Q_{t}}(d y) \\
\leq\|\Phi\|_{0}^{2} \int_{H}\left|\left\langle\Lambda_{t} g, Q_{t}^{-1 / 2} y\right\rangle\right|^{2} N_{Q_{t}}(d y) .
\end{gathered}
$$

Linearity of $I_{t, x}$ is clear; in addition, from this estimate it follows that $I_{t, x}$ is bounded, and $\left\|I_{t, x}\right\| \leq\left\|\Lambda_{t}\right\|\|\Phi\|_{0}$. So inequality (5.10) well be true when we can say that $I_{t, x}=D \mathcal{R}_{t} \Phi(x)$.

Step 2. Let us prove that $\mathcal{R}_{t} \Phi$ is differentiable at $x$ and $I_{t, x}$ is the differential. We have, for $g, h \in H$,

$$
\begin{gathered}
\left\langle\mathcal{R}_{t} \Phi(x+g)-\mathcal{R}_{t} \Phi(x)-I_{t, x} g, h\right\rangle \\
=R_{t} \phi_{h}(x+g)-R_{t} \phi_{h}(x)-\int_{H}\left\langle\Lambda_{t} g, Q_{t}^{-1 / 2} y\right\rangle \phi_{h}\left(e^{A t} x+y\right) N_{Q_{t}}(d y) .
\end{gathered}
$$


Now, by Theorem 6.2 .2 of [6],

$$
\begin{gathered}
R_{t} \phi_{h}(x+g)-R_{t} \phi_{h}(x)=\int_{0}^{1}\left\langle D R_{t} \phi_{h}(x+s g), g\right\rangle d s \\
\int_{0}^{1} \int_{H}\left\langle\Lambda_{t} g, Q_{t}^{-1 / 2} y\right\rangle \phi_{h}\left(e^{A t}(x+s g)+y\right) N_{Q_{t}}(d y) d s \\
\int_{H}\left\langle\Lambda_{t} g, Q_{t}^{-1 / 2} y\right\rangle\left(\int_{0}^{1} \phi_{h}\left(e^{A t}(x+s g)+y\right) d s\right) N_{Q_{t}}(d y) .
\end{gathered}
$$

Thus we have

$$
\begin{gathered}
\left\langle\mathcal{R}_{t} \Phi(x+g)-\mathcal{R}_{t} \Phi(x)-I_{t, x} g, h\right\rangle \\
\int_{H}\left\langle\Lambda_{t} g, Q_{t}^{-1 / 2} y\right\rangle\left[\int_{0}^{1} \phi_{h}\left(e^{A t}(x+s g)+y\right)-\phi_{h}\left(e^{t A} x+y\right) d s\right] N_{Q_{t}}(d y) \\
\leq\left(\int_{H}\left\langle\Lambda_{t} g, Q_{t}^{-1 / 2} y\right\rangle^{2} N_{Q_{t}}(d y)\right)^{1 / 2} \\
\left(\int_{H}\left[\int_{0}^{1} \phi_{h}\left(e^{A t}(x+s g)+y\right)-\phi_{h}\left(e^{t A} x+y\right) d s\right]^{2} N_{Q_{t}}(d y)\right)^{1 / 2} \\
\leq|\Lambda g| \omega_{t}(g) \leq\left\|\Lambda_{t}\right\||| g \mid \omega_{t}(g)
\end{gathered}
$$

where

$$
\omega_{t}(g)=\sup _{s \in[0,1], x, y \in H}\left|\Phi\left(e^{t A}(x+s g)+y\right)-\Phi\left(e^{t A} x+y\right)\right| .
$$

Since $\Phi \in U C_{b}(H, H)$,

$$
\lim _{g \rightarrow 0} \omega_{t}(g)=0
$$

and thus $\mathcal{R}_{t} \Phi$ is differentiable at $x$ with differential $I_{t, x}$. One can check that the differential is uniformly continuous in $x$. Clearly, by (5.10), it is also bounded. Thus we have proved $\mathcal{R}_{t} \Phi \in U C_{b}^{1}(H, H)$ and all claims about $D \mathcal{R}_{t} \Phi$.

Step 3. For given $t, x$ let us analyze the right-hand-side of (5.9). Following [6], Lemma 6.2 .7 , for every bounded measurable $\varphi: H \rightarrow \mathbb{R}$, let us introduce the linear operator $G_{\varphi}^{t, x}$ in $H$ defined as (we use different notations for the Gaussian measure with respect to the quoted reference)

$$
\left\langle G_{\varphi}^{t, x} \alpha, \beta\right\rangle=\int_{H}\left[\left\langle\alpha, Q_{t}^{-1 / 2} y\right\rangle\left\langle\beta, Q_{t}^{-1 / 2} y\right\rangle-\langle\alpha, \beta\rangle\right] \varphi\left(e^{t A} x+y\right) N_{Q_{t}}(d y) .
$$

It is prove in [6] that $G_{\varphi}^{t, x}$ is even Hilbert-Schmidt, with Hilbert-Schmidt norm bounded by $2\|\varphi\|_{0}$. Therefore, in particular, $G_{\varphi}^{t, x}$ is a bounded linear operator with norm

$$
\left\|G_{\varphi}^{t, x}\right\| \leq 2\|\varphi\|_{0}
$$

To understand the right-hand-side of (5.9), let us introduce the linear mapping in $H$

$$
k \mapsto J_{t, x, g} k:=\int_{H}\left[\left\langle\Lambda_{t} g, Q_{t}^{-1 / 2} y\right\rangle\left\langle\Lambda_{t} k, Q_{t}^{-1 / 2} y\right\rangle-\left\langle\Lambda_{t} g, \Lambda_{t} k\right\rangle\right] \Phi\left(e^{t A} x+y\right) N_{Q_{t}}(d y) .
$$


We have

$$
\begin{gathered}
\left|\left\langle J_{t, x, g} k, h\right\rangle\left\|=\left|\left\langle G_{\varphi_{h}}^{t, x} \Lambda_{t} g, \lambda_{t} k\right\rangle\right| \leq\right\| G_{\varphi_{h}}^{t, x}\right|\left\|\left|\Lambda_{t} g\right|\left|\Lambda_{t} k\right| \leq 2\right\| \varphi_{h}\left\|_{0}\right\| \Lambda_{t} \|^{2}|g||k| \\
\leq 2\left\|\Lambda_{t}\right\|^{2}\|\Phi\|_{0}|g||k \| h| .
\end{gathered}
$$

Thus $J_{t, x, g}$ is bounded and

$$
\left\|J_{t, x, g}\right\| \leq 2\left\|\Lambda_{t}\right\|^{2}\|\Phi\|_{0}|g| .
$$

Therefor $g \mapsto J_{t, x, g}$ is a bounded linear operator from $H$ to $L(H, H)$, denoted by $J_{t, x}$ in the sequel of the proof (we have $J_{t, x} g=J_{t, x, g}$ ), and

$$
\left\|J_{t, x}\right\|_{L(H, L(, H, H))} \leq 2\left\|\Lambda_{t}\right\|^{2}\|\Phi\|_{0} .
$$

If we prove that $J_{t, x}$ is $D^{2} \mathcal{R}_{t} \Phi(x)$, we have also proved inequality (5.11).

The proof of (5.12) is similar and based on the Hilbert-Schimdt property mentioned above.

Step 4. Given $t, x$, let us prove that $D \mathcal{R}_{t} \Phi$ is differentiable at $x$, and its differential is $J_{t, x}$. Recall that $\left\langle D \mathcal{R}_{t} \Phi(x) g, h\right\rangle$ is equal to $\left\langle D R_{t} \phi_{h}(x), g\right\rangle$. We have, for $g, h, k \in H$,

$$
\left\langle\left[D \mathcal{R}_{t} \Phi(x+g)-D \mathcal{R}_{t} \Phi(x)\right] k, h\right\rangle=\left\langle D R_{t} \phi_{h}(x+g)-D R_{t} \phi_{h}(x), k\right\rangle
$$

hence, by Proposition 6.2.2 of [6] this equals,

$$
\begin{gathered}
\int_{0}^{1}\left\langle D^{2} R_{t} \varphi_{h}(x+s g) g, k\right\rangle d s \\
=\int_{0}^{1} \int_{H}\left[\left\langle\Lambda_{t} g, Q_{t}^{-1 / 2} y\right\rangle\left\langle\Lambda_{t} k, Q_{t}^{-1 / 2} y\right\rangle-\left\langle\Lambda_{t} g, \Lambda_{t} k\right\rangle\right] \phi_{h}\left(e^{t A}(x s g)+y\right) N_{Q_{t}}(d y) d s \\
=\int_{H}\left[\left\langle\Lambda_{t} g, Q_{t}^{-1 / 2} y\right\rangle\left\langle\Lambda_{t} k, Q_{t}^{-1 / 2} y\right\rangle-\left\langle\Lambda_{t} g, \Lambda_{t} k\right\rangle\right]\left(\int_{0}^{1} \phi_{h}\left(e^{t A}(x s g)+y\right) d s\right) N_{Q_{t}}(d y) .
\end{gathered}
$$

Moreover,

$$
\left\langle\left[J_{t, x} g\right] k, h\right\rangle=\int_{H}\left[\left\langle\Lambda_{t} g, Q_{t}^{-1 / 2} y\right\rangle\left\langle\Lambda_{t} k, Q_{t}^{-1 / 2} y\right\rangle-\left\langle\Lambda_{t} g, \Lambda_{t} k\right\rangle\right] \phi_{h}\left(e^{t A} x+y\right) N_{Q_{t}}(d y) .
$$

Therefore

$$
\begin{gathered}
\left\langle\left[D \mathcal{R}_{t} \Phi(x+g)-D \mathcal{R}_{t} \Phi(x)-J_{t, x} g\right] k, h\right\rangle \\
\int_{H}\left[\left\langle\Lambda_{t} g, Q_{t}^{-1 / 2} y\right\rangle\left\langle\Lambda_{t} k, Q_{t}^{-1 / 2} y\right\rangle-\left\langle\Lambda_{t} g, \Lambda_{t} k\right\rangle\right] \psi_{t, x, g, h}(y) N_{Q_{t}}(d y) \\
=\left\langle G_{\psi_{t, x, g, h}} \Lambda_{t} g, \Lambda_{t} k\right\rangle
\end{gathered}
$$

where

$$
\psi_{t, x, g, h}(y)=\int_{0}^{1}\left[\varphi_{h}\left(e^{t A}(x+s g)+y\right)-\varphi_{h}\left(e^{t A} x+y\right)\right] d s
$$

Hence, by Lemma 6.2 .7 of [6],

$$
\begin{aligned}
\left|\left\langle\left[D \mathcal{R}_{t} \Phi(x+g)-D \mathcal{R}_{t} \Phi(x)-J_{t, x} g\right] k, h\right\rangle\right| & =\left|\left\langle G_{\psi_{t, x, g, h}} \Lambda_{t} g, \Lambda_{t} k\right\rangle\right| \\
& \leq 2\left\|\psi_{t, x, g, h}\right\|_{0}\left\|\Lambda_{t}\right\|^{2}|g||k| .
\end{aligned}
$$


But

$$
\left|\psi_{t, x, g, h}(y) \leq\right| h\left|\int_{0}^{1}\left[\Phi\left(e^{t A}(x+s g)+y\right)-\Phi\left(e^{t A} x+y\right)\right] d s l e q\right| h \mid \omega_{t}(g)
$$

as in step 2. Therefore $D \mathcal{R}_{t} \Phi$ is differentiable at $x$ and $D^{2} \mathcal{R}_{t} \Phi$ is $J_{t, x}$. One can check that $D^{2} \mathcal{R}_{t} \Phi$ is uniformly continuous in $x$. By (5.11), it is also bounded. We have proved $\mathcal{R}_{t} \Phi \in U C_{b}^{2}(H, H)$ and all claims about $D^{2} \mathcal{R}_{t} \Phi$ when $\Phi \in U C_{b}(H, H)$. The proof of the claims on $D^{2} \mathcal{R}_{t} \Phi$ when $\Phi \in U C_{b}^{1}(H, H)$ is similar and based on Proposition 6.2.9 of [6]. We do not give the details. The proof is complete.

\subsection{Non homogenuous Kolmogorov equation}

In this section we assume the conditions on $A, B, Q$ stated in the introduction and Section 5.1.1 and we study the sequence on non-homogeneous Kolmogorov equations in $H$

$$
\begin{aligned}
\frac{\partial U_{n}}{\partial t} & =\frac{1}{2} \operatorname{Tr}\left(D^{2} U_{n} Q\right)+\left\langle A x, D U_{n}\right\rangle+\left\langle B, D U_{n}\right\rangle+G_{n} \\
U_{n}(0, x) & =0
\end{aligned}
$$

where $G_{n}$ are the components of a function $G \in C\left([0, T] ; C_{b}^{\alpha}(H, H)\right)$. In this section we use forward notations for the PDE, for the sake of simplicity. The final result will apply to the backward PDE (5.5) (in particular, the assumption $B \in C\left([0, T] ; C_{b}^{\alpha}(H, H)\right)$ is invariant by time reversal).

We also show that the $H$-valued function $U(t, x)=\sum_{n} U_{n}(t, x) e_{n}$ has a meaning and we analyze its properties.

We interpret the PDE (5.13) as the integral equation

$$
U_{n}(t, x)=\int_{0}^{t} R_{t-s}\left(\left\langle B(s), D U_{n}(s)\right\rangle+G_{n}(s)\right)(x) d s .
$$

Here we write $B(s)$ for $B(s, \cdot)$ and so on. Let us introduce alse the $H$-valued equation

$$
U(t, x)=\int_{0}^{t} \mathcal{R}_{t-s}(\langle B(s), D\rangle U(s)+G(s))(x) d s .
$$

where we have denoted $\sum_{n} e_{n}\left\langle B(s), D U_{n}(s)\right\rangle$ by $\langle B(s), D\rangle U(s)$.

We can state the main result of this section. The regularity we prove for $U$ is not optimal, and the theorem is restricted for simplicity of exposition to small $T^{\text {'s }} \mathrm{s}$.

Theorem 5.3 Under the assumptions of Section 5.1.1, given

$$
B, G \in C\left([0, T] ; U C_{b}(H, H)\right),
$$

for $T$ small enough there exists a unique solution $U$ of equation (5.15) in $C\left([0, T] ; U C_{b}^{1}(H, H)\right)$. If we put $K_{T}:=\|D U\|_{0}$, then

$$
\lim _{T \rightarrow 0} K_{T}=0 .
$$

Moreover, $D U \in C\left([0, T] ; C_{b}^{\theta}(H, H)\right)$, $\theta$ such that assumption (5.4) hold. 
If in addition $B, G \in C\left([0, T] ; C_{b}^{\alpha}(H, H)\right)$ for some $\alpha>0$, then $U \in C\left([0, T] ; U C_{b}^{2}(H, H)\right)$. Finally, there is a constant $C_{T}>0$ such that

$$
\left\|D^{2} U_{n}\right\|_{0} \leq C_{T}\left\|G_{n}\right\|_{\alpha}
$$

for every $n \in \mathbb{N}$.

Proof. Step 1. Consider the map $\mathcal{L}$ defined as

$$
\mathcal{L} U(t, x)=\int_{0}^{t} \mathcal{R}_{t-s}(\langle B(s), D\rangle U(s)+G(s))(x) d s .
$$

It is defined on functions $U \in C\left([0, T] ; U C_{b}^{1}(H, H)\right)$. It is easy to check that $\mathcal{L} U \in C\left([0, T] ; U C_{b}(H, H)\right)$. But we also have the bound

$$
\begin{gathered}
\int_{0}^{t}\left\|D \mathcal{R}_{t-s}(\langle B(s), D\rangle U(s)+G(s))(x)\right\| d s \\
\leq \int_{0}^{t}\left\|\Lambda_{t-s}\right\|\|\langle B(s), D\rangle U(s)+G(s)\|_{0} d s \\
\quad \leq\left(\|B\|_{0}\|D U\|_{0}+\|G\|_{0}\right) \int_{0}^{t}\left\|\Lambda_{s}\right\| d s
\end{gathered}
$$

which implies $\mathcal{L} U \in C\left([0, T] ; U C_{b}^{1}(H, H)\right)$ and

$$
D \mathcal{L} U(t, x)=\int_{0}^{t} D \mathcal{R}_{t-s}(\langle B(s), D\rangle U(s)+G(s))(x) d s .
$$

Since $\lim _{T \rightarrow 0} \int_{0}^{T}\|\Lambda\| d s=0$, and the map $\mathcal{L}$ is linear, it is a contraction in $C\left([0, T] ; U C_{b}^{1}(H, H)\right)$ for sufficiently small $T$ (one has to use also an estimate on $U$ in the norm of $C\left([0, T] ; U C_{b}(H, H)\right)$ ). Moreover, if $U$ is a solution, then

$$
D U(t, x)=\int_{0}^{t} D \mathcal{R}_{t-s}(\langle B(s), D\rangle U(s)+G(s))(x) d s
$$

hence

$$
\|D U\|_{0} \leq\left(\|B\|_{0}\|D U\|_{0}+\|G\|_{0}\right) \int_{0}^{t}\left\|\Lambda_{s}\right\| d s
$$

hence, for $T$ such that $\|B\|_{0} \int_{0}^{T}\|\Lambda\| d s \leq 1 / 2$ we have

$$
\frac{1}{2}\|D U\|_{0} \leq\|G\|_{0} \int_{0}^{t}\left\|\Lambda_{s}\right\| d s
$$

which proves $\lim _{T \rightarrow 0} K_{T}=0$. We have proved the first claims of the theorem.

Step 2. Let us recall a result from interpolation theory developed in [6], Chapter 2. From Theorem 2.3.3 and the remarks at the beginning of section 2.3.3, for every $\theta_{1}(0,1)$ there is a constant $C_{\theta}>0$ such that

$$
\|\varphi\|_{\theta} \leq C_{\theta}\|\varphi\|_{0}^{1-\theta}\|\varphi\|_{1}^{\theta}
$$


for every $\varphi \in U C_{b}^{1}(H, \mathbb{R})$. The same result is true for $\Phi \in U C_{b}^{1}(H, H)$. Indeed, for every $h \in H$ the function $\varphi_{h}=\langle\Phi(\cdot), h\rangle$ belongs to $U C_{b}^{1}(H, \mathbb{R})$, hence

$$
\left|\langle\Phi(x)-\Phi(y), h\rangle \leq C_{\theta}\left\|\varphi_{h}\right\|_{0}^{1-\theta}\left\|\varphi_{h}\right\|_{1}^{\theta}\right| h \| x-\left.y\right|^{\theta} .
$$

But $\left\|\varphi_{h}\right\|_{0} \leq\|\Phi\|_{0}|h|$ and $\left\|\varphi_{h}\right\|_{1} \leq\|\Phi\|_{1}|h|$. Hence

$$
\left|\langle\Phi(x)-\Phi(y), h\rangle \leq C_{\theta}\|\Phi\|_{0}^{1-\theta}\|\Phi\|_{1}^{\theta}\right| h \| x-\left.y\right|^{\theta}
$$

which implies

$$
\|\Phi\|_{\theta} \leq C_{\theta}\|\Phi\|_{0}^{1-\theta}\|\Phi\|_{1}^{\theta}
$$

We also have

$$
\|\Phi\|_{\theta} \leq C_{\theta}\|\Phi\|_{0}^{1-\theta}\|D \Phi\|_{0}^{\theta}+C_{\theta}\|\Phi\|_{0} .
$$

Similary, if $\Phi \in U C_{b}^{2}(H, H)$, we have

$$
\|D \Phi\|_{\theta} \leq C_{\theta}\|D \Phi\|_{0}^{1-\theta}\left\|D^{2} \Phi\right\|_{0}^{\theta}+C_{\theta}\|D \Phi\|_{0} .
$$

Step 3. Let us apply the previous interpolation inequality to $\mathcal{R}_{t} \Phi, \Phi \in U C_{b}(H, H)$, $t \geq 0$, with $\theta \in(0,1)$ :

$$
\begin{aligned}
\left\|D \mathcal{R}_{t} \Phi\right\|_{\theta} & \leq C_{\theta}\left\|D \mathcal{R}_{t} \Phi\right\|_{0}^{1-\theta}\left\|D^{2} \mathcal{R}_{t} \Phi\right\|_{0}^{\theta}+C_{\theta}\left\|D \mathcal{R}_{t} \Phi\right\|_{0} \\
& \leq C_{\theta}\left(\left\|\Lambda_{t}\right\|\|\Phi\|_{0}\right)^{1-\theta}\left(\sqrt{2}\left\|\Lambda_{t}\right\|^{2}\|\Phi\|_{0}\right)^{\theta}+C_{\theta}\left\|\Lambda_{t}\right\|\|\Phi\|_{0} \\
& \leq C_{\theta}^{\prime}\left(\left\|\Lambda_{t}\right\|^{1+\theta}+1\right)\|\Phi\|_{0}
\end{aligned}
$$

for a new constant $C_{\theta}^{\prime}>0$, where we have used inequalities (5.10) and (5.11). Thus from (5.17) we have

$$
\begin{aligned}
\|D U(t)\|_{\theta} & \leq \int_{0}^{t} \| D \mathcal{R}_{t-s}\left(\langle B(s), D\rangle U(s)+G(s) \|_{\theta} d s\right. \\
& \leq \int_{0}^{t} C_{\theta}^{\prime}\left(\left\|\Lambda_{t}\right\|^{1+\theta}+1\right)\|\langle B(s), D\rangle U(s)+G(s)\|_{0} d s \\
& =C_{\theta}^{\prime}\|\langle B, D\rangle U+G\|_{0} \int_{0}^{t}\left(\left\|\Lambda_{t}\right\|^{1+\theta}+1\right) d s .
\end{aligned}
$$

If $\theta$ satisfies the assumption of section 5.1.1, namely $\int_{0}^{t}\left\|\Lambda_{t}\right\|^{1+\theta} d s<\infty$, we deduce that $D U(t) \in C_{b}^{\theta}(H, H)$ for each $t \in[0, T]$. Easily one can check that $D U \in C\left([0, T], C_{b}^{\theta}(H, H)\right)$.

Step 4. Assume now $B, G \in C\left([0, T], C_{b}^{\alpha}(H, H)\right)$. Since $\theta \geq \alpha$ (see section 5.1.1), we know that $\langle B, D\rangle U+G \in C\left([, T], C_{b}^{\alpha}(H, H)\right)$. We use again an interpolation result of [6], see the proof of Lemma 6.4.1: there exists $C_{\alpha}^{\prime \prime}>0$ such that

$$
\left\|D^{2} R_{t} \varphi(x)\right\| \leq C_{\alpha}^{\prime \prime}\left\|\Lambda_{t}\right\|^{2-\alpha}\|\varphi\|_{\alpha}
$$

for all $\varphi \in C_{b}^{\alpha}(H, \mathbb{R})$. It follows that

$$
\left\|D^{2} \mathcal{R}_{t} \Phi(x)\right\| \leq C_{\alpha}^{\prime \prime}\left\|\Lambda_{t}\right\|^{2-\alpha}\|\Phi\|_{\alpha}
$$


for all $\Phi \in C_{b}^{\alpha}(H, H)$.

Using these fact, from (5.17) we have

$$
\begin{aligned}
\left\|D^{2} U(t)\right\| & \leq \int_{0}^{t} \| D^{2} \mathcal{R}_{t-s}(\langle B(s), D\rangle U(s)+G(s) \| d s \\
& \leq \int_{0}^{t} C_{\theta}^{\prime \prime}\left\|\Lambda_{t}\right\|^{2-\alpha}\|\langle B(s), D\rangle U(s)+G(s)\|_{\alpha} d s \\
& =C_{\theta}^{\prime}\|\langle B, D\rangle U+G\|_{\alpha} \int_{0}^{t}\left\|\Lambda_{t}\right\|^{2-\alpha} d s
\end{aligned}
$$

We have $\int_{0}^{t}\left\|\Lambda_{t}\right\|^{2-\alpha} d s<\infty$ (see section 5.1.1), hence $U \in C\left([0, T] ; U C_{b}^{2}(H, H)\right.$ ).

Step 5. From (5.17) or directly from equation (5.14) we have

$$
D U_{n}(t, x)=\int_{0}^{t} D R_{t-s}\left(\left\langle B(s), D U_{n}(s)\right\rangle+G_{n}(s)\right)(x) d s
$$

and thus

$$
D^{2} U_{n}(t, x)=\int_{0}^{t} D^{2} R_{t-s}\left(\left\langle B(s), D U_{n}(s)\right\rangle+G_{n}(s)\right)(x) d s .
$$

From the first one of these identities, with the same computations of step 1, we get (on the interval $[0, T]$ found in step 1 )

$$
\left\|D U_{n}\right\|_{0} \leq C_{1}\left\|G_{n}\right\|_{0} .
$$

As in step 3, we get

$$
\left\|D U_{n}\right\|_{\alpha} \leq C_{2}\left\|\left\langle B, D U_{n}\right\rangle+G_{n}\right\|_{0}
$$

and thus

$$
\left\|D U_{n}\right\|_{\alpha} \leq\left(C_{1} C_{2}\|B\|_{0}+C_{2}\right)\left\|G_{n}\right\|_{0} .
$$

Finally, from the equation for $D^{2} U_{n}(t, x)$, exactly as in step 4 , we prove

$$
\left\|D^{2} U_{n}\right\|_{0} \leq C_{3}\left\|\left\langle B, D U_{n}\right\rangle+G_{n}\right\|_{\alpha} .
$$

Putting together these estimates, we obtain (5.16). The proof is complete.

\subsection{Malliavin Differentiability}

\subsubsection{Strong Uniqueness}

We fix a filtered probability space $(\Omega, \mathcal{F}, P),\left\{\mathcal{F}_{t}\right\}_{t \in[0, T]}$ such that $W$ is a $\mathcal{F}_{t}$-cylindrical Brownian motion on $H$. A mild solution of equation (5.1) is a process $X=(X)_{t \in[0, T]}$, which is an $\mathcal{F}_{t}$-adapted continuous process in $H$ and satisfies

$$
X_{t}=e^{t A} x+\int_{0}^{t} e^{(t-s) A} B\left(s, X_{s}\right) d s+\int_{0}^{t} e^{(t-s) A} \sqrt{Q} d W_{s} .
$$

The stochastic integral is well defined since we have assumed $Q_{t}$ is of trace class.

The following rewriting is essential to our estimates: 
Lemma 5.4 Under the assumptions of Section 5.1.1, let $U$ be the solution given by Theorem 5.3. If $X=(X)_{t \in[0, T]}$ is a mild solution of equation (5.1), then the equation (5.6) is satisfied.

Proof. Having now Theorem 5.3, the proof is the one given in Section 5.2. The only point is the application of Itô's formula. In order to use elementary versions of it, one can introduce the approximations

$$
d X_{t}^{j, h}=A_{j} X_{t}^{j, h} d t+B\left(t, X_{t}^{j, h}\right) d t+P_{h} \sqrt{Q} d W_{t}, X_{0}^{j, h}=x
$$

where $A_{j}$ are the Yosida approximations of $A, P_{h} x=\sum_{i=1}^{h} x_{i} e_{i}$. The computations of Section 5.2 can be done on these approximations and then one can pass to the limit in the final equation. We omit the details which are classical.

Using the previous lemma we proceed to prove pathwise uniqueness for the equation (5.1).

Theorem 5.5 There exists a $T>0$ such that pathwise uniqueness holds for (5.1) on $[0, T]$. That is, if $X^{1}$ and $X^{2}$ are two mild solutions, then we have for leb $\times P$ almost all $(t, x) \in$ $[0, T] \times \Omega, X_{t}^{1}(\omega)=X_{t}^{2}(\omega)$.

Proof. Assume $X^{1}$ and $X^{2}$ are two milds solutions, and define $V_{t}=X_{t}^{1}-X_{t}^{2}$. Then, by Lemma 5.4 we have

$$
\begin{aligned}
\int_{0}^{T}\left|V_{t}\right|^{2} d t & \leq 3 \int_{0}^{T}\left|U\left(t, X_{t}^{1}\right)-U\left(t, X_{t}^{2}\right)\right|^{2} d t \\
& +3 \int_{0}^{T}\left|\int_{0}^{t} A e^{(t-s) A}\left[U\left(s, X_{s}^{1}\right)-U\left(s, X_{s}^{2}\right)\right] d s\right|^{2} d t \\
& +3 \int_{0}^{T}\left|I_{t}\left(X^{1}\right)-I_{t}\left(X^{2}\right)\right|^{2} d t
\end{aligned}
$$

From Theorem 5.3 we have

$$
\left|U\left(t, X_{t}^{1}\right)-U\left(t, X_{t}^{2}\right)\right| \leq K_{T}\left|X_{t}^{1}-X_{t}^{2}\right|, t \in[0, T] .
$$

To deal with the second term we use the maximal inequality

$$
\left\|\int_{0}^{\cdot} A e^{(\cdot-s) A} f(s) d s\right\|_{L^{2}(0, T ; H)}^{2} \leq C_{T}\|f\|_{L^{2}(0, T ; H)}^{2}
$$

where $C_{T}$ is a constant independent of $f$. Notice, however, that $C_{T}$ does not converge to 0 as $T \rightarrow 0$. We then make the following estimate:

$$
\int_{0}^{T}\left|V_{t}\right|^{2} d t \leq\left(3+3 C_{T}\right) K_{T} \int_{0}^{T}\left|V_{t}\right|^{2} d t+3 \int_{0}^{T}\left|I_{t}\left(X^{1}\right)-I_{t}\left(X^{2}\right)\right|^{2} d t .
$$

For $T$ small enough we thus have

$$
\int_{0}^{T}\left|V_{t}\right|^{2} d t \leq 6 \int_{0}^{T}\left|I_{t}\left(X^{1}\right)-I_{t}\left(X^{2}\right)\right|^{2} d t
$$

and in particular

$$
\int_{0}^{T} E\left[\left|V_{t}\right|^{2}\right] d t \leq 6 \int_{0}^{T} E\left[\left|I_{t}\left(X^{1}\right)-I_{t}\left(X^{2}\right)\right|^{2}\right] d t
$$


The proof will be complete once we find an estimate on the right-and side of the previous inquality. We have

$$
\int_{0}^{T} E\left[\left|I_{t}\left(X^{1}\right)-I_{t}\left(X^{2}\right)\right|^{2}\right] d t=\int_{0}^{t}\left\|e^{(t-s) A}\left(D U\left(s, X_{s}^{1}\right)-D U\left(s, X_{s}^{2}\right)\right) \sqrt{Q}\right\|_{H S}^{2} d s .
$$

For the kernel, we write

$$
\begin{array}{r}
\left\|e^{(t-s) A}\left(D U\left(s, X_{s}^{1}\right)-D U\left(s, X_{s}^{2}\right)\right) \sqrt{Q}\right\|_{H S}^{2} \\
=\sum_{n, h \geq 1}\left\langle e^{(t-s) A}\left(D U\left(s, X_{s}^{1}\right)-D U\left(s, X_{s}^{2}\right)\right) \sqrt{Q} e_{h}, e_{n}\right\rangle^{2} \\
=\sum_{n, h \geq 1} e^{-2(t-s) \alpha_{n}}\left\langle\left(D U_{n}\left(s, X_{s}^{1}\right)-D U_{n}\left(s, X_{s}^{2}\right)\right), \sqrt{Q} e_{h}\right\rangle^{2} \\
=\sum_{n \geq 1} e^{-2(t-s) \alpha_{n}} \sum_{h \geq 1}\left\langle\sqrt{Q}\left(D U_{n}\left(s, X_{s}^{1}\right)-D U_{n}\left(s, X_{s}^{2}\right)\right), e_{h}\right\rangle^{2} \\
\leq\|Q\| \sum_{n \geq 1} e^{-2(t-s) \alpha_{n}}\left|D U_{n}\left(s, X_{s}^{1}\right)-D U_{n}\left(s, X_{s}^{2}\right)\right|^{2} \\
\leq\|Q\| \sum_{n \geq 1} e^{-2(t-s) \alpha_{n}}\left\|D U_{n}\right\|_{\infty}^{2}\left|X_{s}^{1}-X_{s}^{2}\right|^{2}
\end{array}
$$

From Theorem 5.3 we have

$$
\left\|D U_{n}\right\|_{\infty} \leq C_{T}\left\|B_{n}\right\|_{\alpha}
$$

hence

$$
E\left[\left|I_{t}\left(X^{1}\right)-I_{t}\left(X^{2}\right)\right|^{2}\right] \leq C_{T}^{2}\|Q\| \int_{0}^{t} \sum_{n \geq 1} e^{-2(t-s) \alpha_{n}}\left\|B_{n}\right\|_{\alpha}^{2}\left|V_{s}\right|^{2} d s .
$$

Therefore

$$
\begin{aligned}
E\left[\int_{0}^{T}\left|I_{t}\left(X^{1}\right)-I_{t}\left(X^{2}\right)\right|^{2} d t\right] & \leq C_{T}^{2}\|Q\| \int_{0}^{T} \int_{0}^{t} \sum_{n \geq 1} e^{-2(t-s) \alpha_{n}}\left\|B_{n}\right\|_{\alpha}^{2}\left|V_{s}\right|^{2} d s \\
& \leq C_{T}^{2}\|Q\|\|B\|_{\alpha}^{2} \int_{0}^{T}\left(\int_{s}^{T} \sum_{n \geq 1} e^{-2(t-s) \alpha_{n}} d t\right)\left|V_{s}\right|^{2} d s \\
& \leq C_{t}^{2}\|Q\|\|B\|_{\alpha}^{2}\left(\int_{0}^{T} \sum_{n \geq 1} e^{-2 t \alpha_{n}} d t\right) \int_{0}^{T}\left|V_{s}\right|^{2} d s .
\end{aligned}
$$

By assumption (5.2) we have $\lim _{T \rightarrow 0} \int_{0}^{T} \sum_{n \geq 1} e^{-2 t \alpha_{n}} d t=0$, so that for small enough $T$ we have

which gives the result.

$$
E\left[\int_{0}^{T}\left|V_{s}\right|^{2} d s\right]=0
$$

Notice that by the Yamada-Watanabe theorem, the previous theorem coupled with weak existence is enough to guarantee strong existence of equation (5.1). We will not elaborate further on this here.

In this paper we will however use Malliavin calculus to construct the solution. As a byproduct of the construction method, we will prove that the solution is Malliavin differentiable. 


\subsubsection{Malliavin Differentiability}

In the remainder of this section we want to use a compactness criterion for $L^{2}$-functionals of $W_{t}$ based on Malliavin calculus (see Appendix, Theorem 5.14) to construct Malliavin differentiable mild solutions to (5.1).

To this end we need some definitions and auxiliary results.

Denote by $L_{2}(H)$ the space of Hilbert-Schmidt operators from $H$ into itself with norm $\|\cdot\|_{H S}$. In what follows let $M: D(M) \subset H \longrightarrow H$ be a non-negative self-adjoint operator with existing compact inverse $M^{-1}$. Further consider the space $E$ obtained by completion with respect to the norm $\|\cdot\|_{E}$ given by

$$
\|K\|_{E}^{2}:=\sum_{n \geq 1}\left\|K M e_{n}\right\|^{2}
$$

for $K \in L_{2}(H)$, if defined.

From now on we also assume that $Q^{1 / 2} M$ has a self-adjoint continuous extension to $H$ such that

$$
\left\|e^{(t-u) A} Q^{1 / 2}\right\|_{E}^{2} \leq C \frac{1}{(t-u)^{1-\delta}}
$$

for all $t>u \geq 0$ and

$$
\begin{aligned}
& \left\|e^{\left(t-u_{1}\right) A} Q^{1 / 2}-e^{\left(t-u_{2}\right) A} Q^{1 / 2}\right\|_{E}^{2} \\
\leq & C \frac{1}{\left(t-u_{1}\right)^{1-\delta}}\left|u_{1}-u_{2}\right|^{\mu}
\end{aligned}
$$

for all $t>u_{1}>u_{2} \geq 0$ and some $\mu>0$. Further we also assume that

$$
\int_{H}\left\|e^{\left(u_{1}-u_{2}\right) A} y-y\right\|^{2} N_{Q_{s}}(d y) \leq C\left|u_{1}-u_{2}\right|^{\eta}
$$

for all $u_{1}>u_{2} \geq 0, s \geq 0$ and some $\eta>0$.

Remark 5.6 Since $\|K\|_{H . S .} \leq C\|K\|_{E}$ for all $K \in E$ for a constant $C$ depending on $M$ we also see that

$$
\left\|e^{(t-u) A} Q^{1 / 2}\right\|_{H . S .}^{2} \leq C \frac{1}{(t-u)^{1-\delta}}
$$

for all $t>u \geq 0$,

$$
\begin{aligned}
& \left\|e^{\left(t-u_{1}\right) A} Q^{1 / 2}-e^{\left(t-u_{2}\right) A} Q^{1 / 2}\right\|_{H . S .}^{2} \\
\leq & C \frac{1}{\left(t-u_{1}\right)^{1-\delta}}\left|u_{1}-u_{2}\right|^{\vartheta}
\end{aligned}
$$

for all $t>u_{1}>u_{2} \geq 0$.

The next result shows that if $B$ in (5.1) is "nice" then the $E$-norm of the Malliavin derivative $D_{u} X_{t}$ of $X_{t}$ exists $u$-a.e., $P$-a.e. 
Lemma 5.7 Suppose that $B \in C\left([0, T] ; C_{b}^{1}(H, H)\right)$ in (1). Further assume the conditions (5.18) and (5.19). Then

$$
\begin{aligned}
& E\left[\int_{0}^{T}\left\|D_{u} X_{t}\right\|_{E}^{2} d u\right] \\
\leq & C\left(\frac{1}{\delta} T^{\delta}+\exp \left(2 \sup _{0 \leq r \leq T}\left\|e^{r A}\right\| \sup _{0 \leq s \leq T}\|D B(s, \cdot)\|_{0} T\right)\right)
\end{aligned}
$$

for all $0 \leq t \leq T$.

Proof. Since

$$
X_{t}=e^{t A} x+\int_{0}^{t} e^{(t-s) A} B\left(s, X_{s}\right) d s+\int_{0}^{t} e^{(t-s) A} Q^{1 / 2} d W_{s}, 0 \leq t \leq T,
$$

we find for all $0 \leq u \leq T$ that

$$
D_{u} X_{t}=\int_{u}^{t} e^{(t-s) A} D B\left(s, X_{s}\right) D_{u} X_{s} d s+e^{(t-u) A} Q^{1 / 2}, u \leq t \leq T .
$$

So we obtain by Picard iteration that

$$
\begin{aligned}
& D_{u} X_{t} \\
& e^{(t-u) A} Q^{1 / 2}+\sum_{n \geq 2} \int_{u \leq s_{1}<\ldots<s_{n-1} \leq t} e^{\left(s_{n}-s_{n-1}\right) A} D B\left(s_{n-1}, X_{s_{n-1}}\right) \ldots \\
& e^{\left(s_{2}-s_{1}\right) A} D B\left(s_{1}, X_{s_{1}}\right) e^{\left(s_{1}-u\right) A} Q^{1 / 2} d s_{1} \ldots d s_{n-1}
\end{aligned}
$$

in $L^{2}\left(\Omega ; L_{2}(H)\right)$ for all $u \leq t \leq T$.

Hence it follows from (5.23) $t>u$ that

$$
\begin{aligned}
& \left\|D_{u} X_{t}\right\|_{E} \\
\leq & \left\|e^{(t-u) A} Q^{1 / 2}\right\|_{E}+\sum_{n \geq 2} \int_{u \leq s_{1}<\ldots<s_{n-1} \leq t} \sup _{0 \leq r \leq T}\left\|e^{r A}\right\|\left\|D B\left(s_{n-1}, \cdot\right)\right\|_{0} \ldots \\
& \sup _{0 \leq r \leq T}\left\|e^{r A}\right\|\left\|D B\left(s_{1}, \cdot\right)\right\|_{0}\left\|e^{\left(s_{1}-u\right) A} Q^{1 / 2}\right\|_{E} d s_{1} \ldots d s_{n-1} \\
\leq & \left\|e^{(t-u) A} Q^{1 / 2}\right\|_{E}+\sum_{n \geq 2} \sup _{0 \leq r \leq T}\left\|e^{r A}\right\|^{n-1} \\
& \cdot \frac{1}{(n-1) !} \int_{[u, T]^{n-1}}\left\|D B\left(s_{n-1}, \cdot\right)\right\|_{0} \cdot \ldots \cdot\left\|D B\left(s_{1}, \cdot\right)\right\|_{0}\left\|e^{\left(s_{1}-u\right) A} Q^{1 / 2}\right\|_{E} d s_{1} \ldots d s_{n-1} \\
\leq & \left\|e^{(t-u) A} Q^{1 / 2}\right\|_{E}+C \sum_{n \geq 2} \frac{1}{(n-1) !}\left(\sup _{0 \leq r \leq T}\left\|e^{r A}\right\| \sup _{0 \leq s \leq T}\|D B(s, \cdot)\|_{0}\right)^{n-1}(T-u)^{n-2} \\
& \cdot \int_{u}^{T} \frac{1}{\left(s_{1}-u\right)^{1-\delta}} d s_{1} \\
\leq & \left\|e^{(t-u) A} Q^{1 / 2}\right\|_{E}+C\left(1+\sum_{n \geq 2} \frac{1}{(n-1) !}\left(\sup _{0 \leq r \leq T}\left\|e^{r A}\right\|_{0 \leq s \leq T} \sup _{0 \leq D B(s, \cdot) \|_{0} T}\right)^{n-1}\right) \frac{1}{\delta}(T-u)^{\delta} \frac{1}{T} \\
\leq & \left\|e^{(t-u) A} Q^{1 / 2}\right\|_{E}+C_{T, \delta} \exp \left(\sup _{0 \leq r \leq T}\left\|e^{r A}\right\| \sup _{0 \leq s \leq T}\|D B(s, \cdot)\|_{0} T\right) \cdot
\end{aligned}
$$


So

$$
\begin{aligned}
& \int_{0}^{T}\left\|D_{u} X_{t}\right\|_{E}^{2} d u=\int_{0}^{t}\left\|D_{u} X_{t}\right\|_{E}^{2} d u \\
\leq & C\left(\int_{0}^{t} \frac{1}{(t-u)^{1-\delta}} d u+\exp \left(2 \sup _{0 \leq r \leq T}\left\|e^{r A}\right\| \sup _{0 \leq s \leq T}\|D B(s, \cdot)\|_{0} T\right)\right) \\
= & C\left(\frac{1}{\delta} t^{\delta}+\exp \left(2 \sup _{0 \leq r \leq T}\left\|e^{r A}\right\| \sup _{0 \leq s \leq T}\|D B(s, \cdot)\|_{0} T\right)\right)<\infty .
\end{aligned}
$$

We shall also use the following Lemma

Lemma 5.8 Let $B$ in (5.1) be in $C\left([0, T] ; C_{b}^{1}(H, H)\right)$. Then $X_{t} \in D\left(A^{\gamma / 2}\right) P$-a.e. for all $0<\gamma<1$ and

$$
E\left[\left\|A^{\gamma / 2} X_{t}\right\|^{2}\right] \leq C \frac{1}{t^{\gamma}}\left(1+\|B\|_{0}^{2}\right)
$$

for all $0 \leq t \leq T$.

Proof. Since

$$
X_{t}=e^{t A} x+\int_{0}^{t} e^{(t-s) A} B\left(s, X_{s}\right) d s+\int_{0}^{t} e^{(t-s) A} Q^{1 / 2} d W_{s}, 0 \leq t \leq T,
$$

it is sufficient to prove that

$$
E\left[\left\|Q_{i}\right\|^{2}\right] \leq C_{i} \frac{1}{t^{\gamma}}\left(1+\|B\|_{0}^{2}\right), i=1,2,3,
$$

where

$$
\begin{aligned}
Q_{1}: & =A^{\gamma / 2} e^{t A} x, Q_{2}:=\int_{0}^{t} A^{\gamma / 2} e^{(t-s) A} B\left(s, X_{s}\right) d s, \\
Q_{3}: & =\int_{0}^{t} A^{\gamma / 2} e^{(t-s) A} d W_{s} .
\end{aligned}
$$

Then using the inequality

$$
\left\|A^{\gamma / 2} e^{(t-s) A}\right\| \leq \frac{C_{\gamma}}{(t-s)^{\gamma / 2}}
$$

and Itô's isometry the result follows.

The next Lemma will be crucial for the application of the compactness criterion Theorem 5.14 in the Appendix.

Lemma 5.9 Assume that $B \in C\left([0, T] ; C_{b}^{1}(H, H)\right) \cap C\left([0, T] ; C_{b}^{\alpha}(H, H)\right)$. Let $X$. be the mild solution to (5.1) associated with the coefficient $B$. Then for all $0 \leq \theta \leq T$ there exists $a$ $0<\beta<\frac{1}{2}$ such that

$$
E\left[\int_{0}^{\theta}\left\|D_{u} X_{\theta}\right\|_{E}^{2} d u\right] \leq L_{1}\left(\|B\|_{\alpha}^{2}\right)
$$

and

$$
E\left[\int_{0}^{\theta} \int_{0}^{\theta} \frac{\left\|D_{u_{1}} X_{\theta}-D_{u_{2}} X_{\theta}\right\|_{E}^{2}}{\left|u_{1}-u_{2}\right|^{1+2 \beta}} d u_{1} d u_{2}\right] \leq L_{2}\left(\|B\|_{\alpha}^{2}\right)
$$

where $L_{i}, i=1,2$ are non-negative continuous functions on $\left[0, \frac{1}{V_{T}}\right]$ with $V_{T} \longrightarrow 0$ for $T \longrightarrow 0$. 
Proof By applying the chain rule for the Malliavin derivative (see [13]) we know that

$$
\begin{aligned}
D_{u} X_{t}= & D U\left(t, X_{t}\right) D_{u} X_{t}+\int_{u}^{t} A e^{(t-s) A} D U\left(s, X_{s}\right) D_{u} X_{s} d s \\
& +e^{(t-u) A} Q^{1 / 2}-e^{(t-u) A} D U\left(u, X_{u}\right) Q^{1 / 2} \\
& -\int_{u}^{t} e^{(t-s) A} D^{2} U\left(s, X_{s}\right) Q^{1 / 2} D_{u} X_{s} d W_{s} \\
= & I_{1}+I_{2}+I_{3}+I_{4}+I_{5}
\end{aligned}
$$

for $u \leq t<T \quad P$-a.e., where

$$
\begin{aligned}
& I_{1}:=D U\left(t, X_{t}\right) D_{u} X_{t}, I_{2}:=\int_{u}^{t} A e^{(t-s) A} D U\left(s, X_{s}\right) D_{u} X_{s} d s, I_{3}:=e^{(t-u) A} Q^{1 / 2}, \\
& I_{4}:=-e^{(t-u) A} D U\left(u, X_{u}\right) Q^{1 / 2}, I_{5}:=-\int_{u}^{t} e^{(t-s) A} D^{2} U\left(s, X_{s}\right) Q^{1 / 2} D_{u} X_{s} d W_{s} .
\end{aligned}
$$

We want to use Gronwall's Lemma to show (5.26) and (5.27). To this end we need some estimates of $I_{1}, \ldots, I_{5}$.

1. Estimate for $I_{1}$ : By Lemma 5.7 and the estimates of Theorem 3 we find that

$$
\begin{aligned}
E\left[\left\|I_{1}\right\|_{E}^{2}\right] & \leq\|D U\|_{0}^{2} E\left[\left\|D_{u} X_{t}\right\|_{E}^{2}\right] \\
& \leq K_{T}\|B\|_{0}^{2} E\left[\left\|D_{u} X_{t}\right\|^{2}\right]_{E}<\infty
\end{aligned}
$$

for $t>u$, where $\lim _{T \longrightarrow 0} K_{T}=0$.

2. Estimate of $I_{2}$ : Using the inequalities

$$
\left\|A^{\epsilon} e^{A t}\right\| \leq \frac{C_{\epsilon}}{t^{\epsilon}}
$$

and

$$
\left\|A^{\epsilon} D U\left(s, X_{t}\right)\right\| \leq C_{\varepsilon, T}\|B\|_{0}
$$

for $0<\epsilon<1$ (see [8]) we obtain

$$
\begin{aligned}
& E\left[\left\|I_{2}\right\|_{E}^{2}\right] \\
\leq & (t-u) E\left[\int_{u}^{t}\left\|A e^{(t-s) A} D U\left(s, X_{s}\right)\right\|^{2}\left\|D_{u} X_{s}\right\|_{E}^{2} d s\right. \\
\leq & (t-u) E\left[\int_{u}^{t}\left\|A^{1-\gamma} e^{(t-s) A}\right\|^{2}\left\|A^{\gamma} D U\left(s, X_{s}\right)\right\|^{2}\left\|D_{u} X_{s}\right\|_{E}^{2} d s\right] \\
\leq & (t-u) C_{\varepsilon, T}\|B\|_{0}^{2} E\left[\int_{u}^{t} \frac{C_{\gamma}}{(t-s)^{2(1-\gamma)}}\left\|D_{u} X_{s}\right\|_{E}^{2} d s\right] \\
= & (t-u) C_{\varepsilon, T} C_{\gamma}\|B\|_{0}^{2} \int_{u}^{t} \frac{1}{(t-s)^{2(1-\gamma)}} E\left[\left\|D_{u} X_{s}\right\|_{E}^{2}\right] d s
\end{aligned}
$$

for $1>\gamma>0$ with $2(1-\gamma)<1($ see $(5.25))$.

3. Estimation of $I_{3}$ : We know from (5.18) and (5.19)that

$$
\left\|I_{3}\right\|_{E}^{2} \leq C \frac{1}{(t-u)^{1-\delta}} .
$$


As for the other two estimates we use the notation of the previous section and let

$$
\Phi=\Phi(s):=\langle B(s), D\rangle U(s)-B(s) .
$$

4. Estimate for $I_{4}$ : Because of our assumptions and Theorem 3 we obtain

$$
\begin{aligned}
\left\|I_{4}\right\|_{E}^{2}= & \left\|e^{(t-u) A} D U\left(u, X_{u}\right) Q^{1 / 2}\right\|_{E}^{2} \\
= & \left\|e^{(t-u) A} D U\left(u, X_{u}\right) Q^{1 / 2} M\right\|_{H . S .}^{2} \\
= & \sum_{n, h \geq 1}\left\langle e^{(t-u) A} D U\left(u, X_{u}\right) Q^{1 / 2} M e_{h}, e_{n}\right\rangle^{2} \\
= & \sum_{n, h \geq 1} e^{-2 \alpha_{n}(t-u)}\left\langle D U\left(u, X_{u}\right) Q^{1 / 2} M e_{h}, e_{n}\right\rangle^{2} \\
= & \sum_{n, h \geq 1} e^{-2 \alpha_{n}(t-u)}\left\langle D U_{n}\left(u, X_{u}\right), Q^{1 / 2} M e_{h}\right\rangle^{2} \\
= & \sum_{n \geq 1} e^{-2 \alpha_{n}(t-u)} \sum_{h \geq 1}\left\langle Q^{1 / 2} M D U_{n}\left(u, X_{u}\right), e_{h}\right\rangle^{2} \\
\leq & \sum_{n \geq 1} e^{-2 \alpha_{n}(t-u)}\left\|Q^{1 / 2} M\right\|^{2}\left\|D U_{n}\left(u, X_{u}\right)\right\|^{2} \\
\leq & \left\|Q^{1 / 2} M\right\|^{2} C_{T}\left(\int_{0}^{T}\left\|\Lambda_{s}\right\| d s\right)^{2} \sum_{n \geq 1} e^{-2 \alpha_{n}(t-u)}\left\|B_{n}\right\|_{0}^{2} \\
= & \left\|Q^{1 / 2} M\right\|^{2} C_{T}\left(\int_{0}^{T}\left\|\Lambda_{s}\right\| d s\right)^{2} \cdot \\
& \cdot\left(\sum_{n \geq 1}\left(2 \alpha_{n}(t-u)\right)^{(1-\delta)} 2 e^{-2 \alpha_{n}(t-u)}\left(2 \alpha_{n}(t-u)\right)^{-(1-\delta)}\left\|B_{n}\right\|_{0}^{2}\right) \\
\leq & C_{\delta}\left\|Q^{1 / 2} M\right\|^{2} C_{T}\left(\int_{0}^{T}\left\|\Lambda_{s}\right\| d s\right)^{2}\|B\|_{0}^{2} 2^{-(1-\delta)} \frac{1}{(t-u)^{(1-\delta)}}
\end{aligned}
$$

if $T$ is small enough. Hence

$$
E\left[\left\|I_{4}\right\|_{E}^{2}\right] \leq C_{\delta}\left\|Q^{1 / 2} M\right\|^{2} C_{T}\left(\int_{0}^{T}\left\|\Lambda_{s}\right\| d s\right)^{2}\|B\|_{0}^{2} 2^{-(1-\delta)} \frac{1}{(t-u)^{(1-\delta)}} .
$$

5. Estimate for $I_{5}$ : By our assumptions and the estimates of Theorem 2 we get for fixed 
$r \in \mathbb{N}$

$$
\begin{aligned}
& \sum_{h \geq 1}\left\|e^{(t-s) A} D^{2} U\left(s, X_{s}\right)\left[Q^{1 / 2} e_{h}, D_{u} X_{s} M e_{r}\right]\right\|^{2} \\
= & \sum_{n, h \geq 1}\left\langle e^{(t-s) A} D^{2} U\left(s, X_{s}\right)\left[Q^{1 / 2} e_{h}, D_{u} X_{s} M e_{r}\right], e_{n}\right\rangle^{2} \\
= & \sum_{n, h \geq 1} e^{-2 \alpha_{n}(t-s)}\left\langle D^{2} U\left(s, X_{s}\right)\left[Q^{1 / 2} e_{h}, D_{u} X_{s} M e_{r}\right], e_{n}\right\rangle^{2} \\
= & \sum_{n, h \geq 1} e^{-2 \alpha_{n}(t-s)}\left(D^{2} U_{n}\left(s, X_{s}\right)\left[Q^{1 / 2} e_{h}, D_{u} X_{s} M e_{r}\right]\right)^{2} \\
= & \sum_{n \geq 1} e^{-2 \alpha_{n}(t-s)} \sum_{h \geq 1}\left(D^{2} U_{n}\left(s, X_{s}\right)\left[Q^{1 / 2} e_{h}, D_{u} X_{s} M e_{r}\right]\right)^{2} \\
\leq & \sum_{n \geq 1} e^{-2 \alpha_{n}(t-s)}\|Q\|\left\|D^{2} U_{n}\right\|_{0}^{2}\left\|D_{u} X_{s} M e_{r}\right\|^{2} \\
\leq & C_{T}\|Q\| \sum_{n \geq 1} e^{-2 \alpha_{n}(t-s)}\left\|B_{n}\right\|_{\alpha}^{2}\left\|D_{u} X_{s} M e_{r}\right\|^{2} \\
= & C_{T}\|Q\| \sum_{n \geq 1}\left(2 \alpha_{n}(t-s)\right)^{(1-\delta)} 2 e^{-2 \alpha_{n}(t-s)} . \\
& \cdot\left(2 \alpha_{n}(t-s)\right)^{-(1-\delta)}\left\|B_{n}\right\|_{\alpha}^{2}\left\|D_{u} X_{s} M e_{r}\right\|^{2} \\
\leq & C_{\delta} C_{T}\|Q\| 2^{-(1-\delta)}\|B\|_{\alpha}^{2}\left\|D_{u} X_{s} M e_{r}\right\|^{2} \frac{1}{(t-s)^{1-\delta}}
\end{aligned}
$$

So it follows from the Itô isometry that

$$
\begin{aligned}
& E\left[\left\|I_{5}\right\|_{E}^{2}\right] \\
= & \sum_{r \geq 1} \sum_{h \geq 1} \int_{u}^{t}\left\|e^{(t-s) A} D^{2} U\left(s, X_{s}\right)\left[Q^{1 / 2} e_{h}, D_{u} X_{s} M e_{r}\right]\right\|^{2} d s \\
\leq & C_{\delta} C_{T}\|Q\| 2^{-(1-\delta)}\|B\|_{\alpha}^{2} \int_{u}^{t} \frac{1}{(t-s)^{1-\delta}}\left\|D_{u} X_{s}\right\|_{E}^{2} d s .
\end{aligned}
$$

So using the above estimates we get

$$
\begin{aligned}
& E\left[\left\|D_{u} X_{t}\right\|_{E}^{2}\right] \\
\leq & C\left(K_{T}\|B\|_{\alpha}^{2} E\left[\left\|D_{u} X_{t}\right\|\right]_{E}^{2}+(t-u) C_{\varepsilon, T} C_{\gamma}\|B\|_{\alpha}^{2} \int_{u}^{t} \frac{1}{(t-s)^{2(1-\gamma)}} E\left[\left\|D_{u} X_{s}\right\|_{E}^{2}\right] d s\right. \\
& +C \frac{1}{(t-u)^{1-\delta}}+C_{\delta}\left\|Q^{1 / 2} M\right\|^{2} C_{T}\left(\int_{0}^{T}\left\|\Lambda_{s}\right\| d s\right)^{2}\|B\|_{\alpha}^{2} 2^{-(1-\delta)} \frac{1}{(t-u)^{(1-\delta)}} \\
& \left.+C_{\delta} C_{T}\|Q\| 2^{-(1-\delta)}\|B\|_{\alpha}^{2} \int_{u}^{t} \frac{1}{(t-s)^{1-\delta}}\left\|D_{u} X_{s}\right\|_{E}^{2} d s\right)
\end{aligned}
$$


for $u \leq t \leq T$. Thus

$$
\begin{aligned}
& E\left[\left\|D_{u} X_{t}\right\|_{E}^{2}\right] \\
\leq & \frac{C}{1-C K_{T}\|B\|_{\alpha}^{2}} \cdot \\
& \cdot\left(C \frac{1}{(t-u)^{1-\delta}}+C_{\delta}\left\|Q^{1 / 2} M\right\|^{2} C_{T}\left(\int_{0}^{T}\left\|\Lambda_{s}\right\| d s\right)^{2}\|B\|_{\alpha}^{2} 2^{-(1-\delta)} \frac{1}{(t-u)^{(1-\delta)}}\right) \\
& +\frac{C}{1-C K_{T}\|B\|_{\alpha}^{2}} \cdot \\
& \cdot\left((t-u) C_{\varepsilon, T} C_{\gamma}\|B\|_{\alpha}^{2}+C_{\delta} C_{T}\|Q\| 2^{-(1-\delta)}\|B\|_{\alpha}^{2}\right) \int_{u}^{t} \frac{1}{(t-s)^{1-\delta}}\left\|D_{u} X_{s}\right\|_{E}^{2} d s
\end{aligned}
$$

for $u \leq t \leq T$ with $T$ small enough such that $C K_{T}\|B\|_{0}<1$. Hence by a generalized Lemma of Gronwall for weakly singular kernels (see [1, Theorem 3]) we get

$$
E\left[\left\|D_{u} X_{t}\right\|_{E}^{2}\right] \leq a(t)+\int_{u}^{t} \sum_{n \geq 1} \frac{(g(t) \Gamma(\delta))^{n}}{\Gamma(n \delta)}(t-s)^{n \delta-1} a(s) d s
$$

for $u \leq t \leq T$, where

$$
\begin{aligned}
& a(s) \\
: & =\frac{C}{1-C K_{T}\|B\|_{\alpha}^{2}} \cdot \\
& \cdot\left(C+C_{\delta}\left\|Q^{1 / 2} M\right\|^{2} C_{T}\left(\int_{0}^{T}\left\|\Lambda_{r}\right\| d r\right)^{2}\|B\|_{\alpha}^{2} 2^{-(1-\delta)}\right) \frac{1}{(s-u)^{1-\delta}} \\
& g(t) \\
: & =\frac{C}{1-C K_{T}\|B\|_{\alpha}^{2}}\left((t-u) C_{\varepsilon, T} C_{\gamma}\|B\|_{\alpha}^{2}+C_{\delta} C_{T}\|Q\| 2^{-(1-\delta)}\|B\|_{\alpha}^{2}\right)
\end{aligned}
$$

and where $\Gamma$ is the Gamma function.

Let us now assume that $n_{0} \delta<1$, but $\left(n_{0}+1\right) \delta \geq 1$ for $n_{0} \in \mathbb{N}$.

Therefore by using the following relation based on the Beta function

$$
\int_{u}^{t}(t-s)^{n \delta-1} \frac{1}{(s-u)^{1-\delta}} d s=\frac{\Gamma(n \delta) \Gamma(\delta)}{\Gamma(n \delta+\delta)}(t-u)^{(n+1) \delta-1},
$$

where $\Gamma$ is the Gamma function, we obtain

$$
\begin{aligned}
& E\left[\left\|D_{u} X_{t}\right\|_{E}^{2}\right] \\
\leq & L_{1}\left(\|B\|_{\alpha}\right) \frac{1}{(t-u)^{1-\delta}}+L_{1}\left(\|B\|_{\alpha}\right) \sum_{n \geq 1} \frac{\left(L_{2}\left(\|B\|_{\alpha}\right) \Gamma(\delta)\right)^{n}}{\Gamma(n \delta)} \frac{\Gamma(n \delta) \Gamma(\delta)}{\Gamma(n \delta+\delta)}(t-u)^{(n+1) \delta-1} \\
\leq & L_{1}\left(\|B\|_{\alpha}\right) \frac{1}{(t-u)^{1-\delta}}+L_{1}\left(\|B\|_{\alpha}\right) \sum_{n=1}^{n_{0}-1} \frac{\left(L_{2}\left(\|B\|_{\alpha}\right) \Gamma(\delta)\right)^{n} \Gamma(\delta)}{\Gamma((n+1) \delta)} \frac{1}{(t-u)^{1-(n+1) \delta}} \\
& +L_{1}\left(\|B\|_{\alpha}\right) \sum_{n \geq n_{0}} \frac{\left(L_{2}\left(\|B\|_{\alpha}\right) \Gamma(\delta)\right)^{n} \Gamma(\delta)}{\Gamma((n+1) \delta)}(t-u)^{(n+1) \delta-1},
\end{aligned}
$$


where $L_{1}$ and $L_{2}$ are non-negative continuous functions on $\left[0, \frac{1}{2 C K_{T}}\right]$, where $K_{T} \longrightarrow 0$ for $T \longrightarrow 0$.

Altogether we get

$$
\begin{aligned}
& E\left[\int_{0}^{T}\left\|D_{u} X_{t}\right\|_{E}^{2} d u\right] \\
= & E\left[\int_{0}^{t}\left\|D_{u} X_{t}\right\|_{E}^{2} d u\right] \\
\leq & L_{1}\left(\|B\|_{\alpha}\right) \frac{1}{\delta} t^{\delta}+L_{1}\left(\|B\|_{\alpha}\right) \sum_{n=1}^{n_{0}-1} \frac{\left(L_{2}\left(\|B\|_{\alpha}\right) \Gamma(\delta)\right)^{n} \Gamma(\delta)}{\Gamma((n+1) \delta)} \frac{t^{(n+1) \delta}}{(n+1) \delta} \\
& +L_{1}\left(\|B\|_{\alpha}\right) \sum_{n \geq n_{0}} \frac{\left(L_{2}\left(\|B\|_{\alpha}\right) \Gamma(\delta)\right)^{n} T^{(n+1) \delta-1} \Gamma(\delta)}{\Gamma((n+1) \delta)} t .
\end{aligned}
$$

Let us now show the estimate (5.27). Assume that $t=\theta \geq u_{1}>u_{2} \geq 0$. Then it follows from (5.28)

$$
D_{u_{2}} X_{t}-D_{u_{1}} X_{t}=\sum_{k=1}^{7} J_{k},
$$

where

$$
\begin{aligned}
& J_{1}:=D U\left(t, X_{t}\right)\left(D_{u_{2}} X_{t}-D_{u_{1}} X_{t}\right), \\
& J_{2}:=\int_{u_{2}}^{u_{1}} A e^{(t-s) A} D U\left(s, X_{s}\right) D_{u_{2}} X_{s} d s, \\
& J_{3}:=\int_{u_{1}}^{t} A e^{(t-s) A} D U\left(s, X_{s}\right)\left(D_{u_{2}} X_{s}-D_{u_{1}} X_{s}\right) d s, \\
& J_{4}:=e^{\left(t-u_{2}\right) A} Q^{1 / 2}-e^{\left(t-u_{1}\right) A} Q^{1 / 2} \\
& J_{5}:=-\left(e^{\left(t-u_{2}\right) A} D U\left(u_{2}, X_{u_{2}}\right) Q^{1 / 2}-e^{\left(t-u_{1}\right) A} D U\left(u_{1}, X_{u_{1}}\right) Q^{1 / 2}\right) \\
& J_{6}:=-\int_{u_{2}}^{u_{1}} e^{(t-s) A} D^{2} U\left(s, X_{s}\right) Q^{1 / 2} D_{u_{2}} X_{s} d W_{s} \\
& J_{7}:=-\int_{u_{1}}^{t} e^{(t-s) A} D^{2} U\left(s, X_{s}\right) Q^{1 / 2}\left(D_{u_{2}} X_{s}-D_{u_{1}} X_{s}\right) d W_{s} .
\end{aligned}
$$

Let us first estimate the terms $J_{4}, J_{5}, J_{2}$ and $J_{6}$.

1. Estimation of $J_{4}$ : By assumption we have

$$
\left\|e^{\left(t-u_{2}\right) A} Q^{1 / 2}-e^{\left(t-u_{1}\right) A} Q^{1 / 2}\right\|_{E}^{2} \leq C \frac{1}{\left(t-u_{1}\right)^{1-\delta}}\left|u_{1}-u_{2}\right|^{\mu}
$$

for all $0 \leq u_{2}<u_{1}<t$ and some $0<\mu<1$.

2. Estimate for $J_{5}$ : We can write $J_{5}$ as

$$
J_{5}=T_{1}+T_{2}+T_{3},
$$

where

$$
\begin{aligned}
& T_{1}:=-\left(e^{\left(t-u_{2}\right) A}-e^{\left(t-u_{1}\right) A}\right) D U\left(u_{2}, X_{u_{2}}\right) Q^{1 / 2} \\
& T_{2}:=-e^{\left(t-u_{1}\right) A}\left(\left(D U\left(u_{2}, X_{u_{2}}\right) Q^{1 / 2}-D U\left(u_{2}, X_{u_{1}}\right) Q^{1 / 2}\right)\right. \\
& T_{3}:=-e^{\left(t-u_{1}\right) A}\left(D U\left(u_{2}, X_{u_{1}}\right) Q^{1 / 2}-D U\left(u_{1}, X_{u_{1}}\right) Q^{1 / 2}\right)
\end{aligned}
$$


2.1. $T_{2}$ : Because of our assumptions, Theorem 3 and the mean value theorem we obtain

$$
\begin{aligned}
\left\|T_{2}\right\|_{E}^{2} & =\left\|e^{\left(t-u_{1}\right) A}\left(D U\left(u_{2}, X_{u_{2}}\right) Q^{1 / 2}-D U\left(u_{2}, X_{u_{1}}\right) Q^{1 / 2}\right)\right\|_{E}^{2} \\
& \left.=\| e^{\left(t-u_{1}\right) A}\left(D U\left(u_{2}, X_{u_{2}}\right)-D U\left(u_{2}, X_{u_{1}}\right)\right) Q^{1 / 2} M\right) \|_{H . S .}^{2} \\
& =\sum_{n, h \geq 1}\left\langle e^{\left(t-u_{1}\right) A}\left(D U\left(u_{2}, X_{u_{2}}\right)-D U\left(u_{2}, X_{u_{1}}\right)\right) Q^{1 / 2} M e_{h}, e_{n}\right\rangle^{2} \\
& =\sum_{n, h \geq 1} e^{-2 \alpha_{n}\left(t-u_{1}\right)}\left\langle\left(D U\left(u_{2}, X_{u_{2}}\right)-D U\left(u_{2}, X_{u_{1}}\right)\right) Q^{1 / 2} M e_{h}, e_{n}\right\rangle^{2} \\
& =\sum_{n, h \geq 1} e^{-2 \alpha_{n}\left(t-u_{1}\right)}\left\langle\left(D U_{n}\left(u_{2}, X_{u_{2}}\right)-D U_{n}\left(u_{2}, X_{u_{1}}\right)\right), Q^{1 / 2} M e_{h}\right\rangle^{2} \\
& =\sum_{n \geq 1} e^{-2 \alpha_{n}\left(t-u_{1}\right)} \sum_{h \geq 1}\left\langle Q^{1 / 2} M\left(D U_{n}\left(u_{2}, X_{u_{2}}\right)-D U_{n}\left(u_{2}, X_{u_{1}}\right)\right), e_{h}\right\rangle^{2} \\
& \leq \sum_{n \geq 1} e^{-2 \alpha_{n}\left(t-u_{1}\right)}\left\|Q^{1 / 2} M\right\|^{2}\left\|D U_{n}\left(u_{2}, X_{u_{2}}\right)-D U_{n}\left(u_{2}, X_{u_{1}}\right)\right\|^{2} \\
& \leq \sum_{n \geq 1} e^{-2 \alpha_{n}\left(t-u_{1}\right)}\left\|Q^{1 / 2} M\right\|^{2} \int_{0}^{1}\left\|D^{2} U_{n}\left(u_{2}, X_{u_{2}}+s\left(X_{u_{2}}-X_{u_{1}}\right)\right)\left(X_{u_{2}}-X_{u_{1}}\right)\right\|^{2} d s \\
& \leq \sum_{n \geq 1} e^{-2 \alpha_{n}\left(t-u_{1}\right)}\left\|Q^{1 / 2} M\right\|^{2}\left\|D^{2} U_{n}\right\|_{0}^{2}\left\|X_{u_{2}}-X_{u_{1}}\right\|^{2} \\
& \leq C_{T}\left\|Q^{1 / 2} M\right\|^{2} \sum_{n \geq 1} e^{-2 \alpha_{n}\left(t-u_{1}\right)}\left\|B_{n}\right\|_{\alpha}^{2}\left\|X_{u_{2}}-X_{u_{1}}\right\|^{2} \\
& =C_{T}\left\|Q^{1 / 2} M\right\|^{2} \sum_{n \geq 1}\left(2 \alpha_{n}\left(t-u_{1}\right)\right)^{(1-\delta)} e^{-2 \alpha_{n}\left(t-u_{1}\right)}\left(2 \alpha_{n}\left(t-u_{1}\right)\right)^{-(1-\delta)}\left\|B_{n}\right\|_{\alpha}^{2}\left\|X_{u_{2}}-X_{u_{1}}\right\|^{2} \\
& \leq C_{T}\left\|Q^{1 / 2} M\right\|^{2}\|B\|_{\alpha}^{2} 2^{-(1-\delta)} \frac{1}{\left(t-u_{1}\right)^{1-\delta}\left\|X_{u_{2}}-X_{u_{1}}\right\|^{2} .}
\end{aligned}
$$

Further we have that

$$
\begin{aligned}
& X_{u_{1}}-X_{u_{2}} \\
= & e^{u_{1} A} x-e^{u_{2} A} x+\int_{0}^{u_{1}} e^{\left(u_{1}-s\right) A} B\left(s, X_{s}\right) d s-\int_{0}^{u_{2}} e^{\left(u_{2}-s\right) A} B\left(s, X_{s}\right) d s \\
& +\int_{0}^{u_{1}} e^{\left(u_{1}-s\right) A} Q^{1 / 2} d W_{s}-\int_{0}^{u_{2}} e^{\left(u_{2}-s\right) A} Q^{1 / 2} d W_{s} \\
= & e^{u_{1} A} x-e^{u_{2} A} x+\int_{0}^{u_{2}}\left(e^{\left(u_{1}-s\right) A}-e^{\left(u_{2}-s\right) A}\right) B\left(s, X_{s}\right) d s+\int_{u_{2}}^{u_{1}} e^{\left(u_{1}-s\right) A} B\left(s, X_{s}\right) d s \\
& \int_{0}^{u_{2}}\left(e^{\left(u_{1}-s\right) A}-e^{\left(u_{2}-s\right) A}\right) Q^{1 / 2} d W_{s}+\int_{u_{2}}^{u_{1}} e^{\left(u_{1}-s\right) A} Q^{1 / 2} d W_{s} .
\end{aligned}
$$


On the other hand we find for all $0<\varepsilon<1 / 2$ that

$$
\begin{aligned}
& \left\|\left(e^{\left(u_{1}-s\right) A}-e^{\left(u_{2}-s\right) A}\right) x\right\|^{2} \\
= & \sum_{k \geq 1}\left\langle x, e_{k}\right\rangle^{2}\left(1-e^{-\left(u_{1}-u_{2}\right) \alpha_{k}}\right)^{2} e^{-2\left(u_{2}-s\right) \alpha_{k}} \\
= & \sum_{k \geq 1}\left\langle x, e_{k}\right\rangle^{2}\left(\left(u_{1}-u_{2}\right) \alpha_{k}\right)^{-2 \varepsilon}\left(1-e^{-\left(u_{1}-u_{2}\right) \alpha_{k}}\right)^{2} . \\
& \cdot\left(\left(u_{1}-u_{2}\right) \alpha_{k}\right)^{2 \varepsilon} \frac{\left(u_{2}-s\right)^{2 \varepsilon}}{\left(u_{2}-s\right)^{2 \varepsilon}} e^{-2\left(u_{2}-s\right) \alpha_{k}} \\
\leq & \|x\|^{2}\left(u_{1}-u_{2}\right)^{2 \varepsilon} \frac{1}{\left(u_{2}-s\right)^{2 \varepsilon}} .
\end{aligned}
$$

Hence it follows in connection with (5.22) and (5.30) for $T<1$ that

$$
\begin{aligned}
& E\left[\left\|X_{u_{1}}-X_{u_{2}}\right\|^{2}\right] \\
\leq & C\left(\|x\|^{2} \frac{1}{u_{2}^{2 \varepsilon}}\left(u_{1}-u_{2}\right)^{2 \varepsilon}+\|B\|_{0}^{2} \frac{1}{1-2 \varepsilon} u_{2}^{1-2 \varepsilon}\left(u_{1}-u_{2}\right)^{2 \varepsilon}+\sup _{0 \leq r \leq T}\left\|e^{r A}\right\|^{2}\|B\|_{0}^{2}\left(u_{1}-u_{2}\right)\right. \\
& \left.+\int_{0}^{u_{2}} \frac{1}{\left(u_{2}-s\right)^{1-\delta}}\left(u_{1}-u_{2}\right)^{\vartheta} d s+\int_{u_{2}}^{u_{1}} \frac{1}{\left(u_{1}-s\right)^{1-\delta}} d s\right) \\
= & C\left(\|x\|^{2} \frac{1}{u_{2}^{2 \varepsilon}}\left(u_{1}-u_{2}\right)^{2 \varepsilon}+\|B\|_{0}^{2} \frac{1}{1-2 \varepsilon} u_{2}^{1-2 \varepsilon}\left(u_{1}-u_{2}\right)^{2 \varepsilon}+\sup _{0 \leq r \leq T}\left\|e^{r A}\right\|^{2}\|B\|_{0}^{2}\left(u_{1}-u_{2}\right)\right. \\
& \left.+\frac{1}{\delta} u_{2}^{\delta}\left(u_{1}-u_{2}\right)^{\vartheta}+\frac{1}{\delta}\left(u_{1}-u_{2}\right)^{\delta}\right) \\
\leq & H\left(\|B\|_{0}\right) \frac{1}{u_{2}^{2 \varepsilon}}\left(u_{1}-u_{2}\right)^{2 \varepsilon \wedge \delta \wedge 1},
\end{aligned}
$$

where $H$ is a non-negative continuous function on $[0, \infty)$.

Therefore we have

$$
\begin{aligned}
& E\left[\left\|T_{2}\right\|^{2}\right] \\
\leq & C_{T}\left\|Q^{1 / 2} M\right\|^{2}\|B\|_{\alpha}^{2} H\left(\|B\|_{0}\right) 2^{-(1-\delta)} \frac{1}{\left(t-u_{1}\right)^{1-\delta}} \frac{1}{u_{2}^{2 \varepsilon}}\left(u_{1}-u_{2}\right)^{2 \varepsilon \wedge \delta \wedge 1} .
\end{aligned}
$$

for all $u_{1} \geq u_{2}$.

2.2. $T_{3}$ : We know that

$$
\begin{aligned}
& -e^{\left(t-u_{1}\right) A}\left(D U\left(u_{2}, X_{u_{1}}\right) Q^{1 / 2}-D U\left(u_{1}, X_{u_{1}}\right) Q^{1 / 2}\right) h \\
= & \int_{0}^{u_{1}} e^{\left(t-u_{1}\right) A}\left[D \mathcal{R}_{u_{1}-r}(\Phi)\left(X_{u_{1}}\right) Q^{1 / 2} h\right] d r-\int_{0}^{u_{2}} e^{\left(t-u_{1}\right) A}\left[D \mathcal{R}_{u_{2}-r}(\Phi)\left(X_{u_{1}}\right) Q^{1 / 2} h\right] d r \\
= & G_{1}+G_{2},
\end{aligned}
$$

where

$$
\begin{aligned}
& G_{1}:=\int_{u_{2}}^{u_{1}} e^{\left(t-u_{1}\right) A}\left[D \mathcal{R}_{u_{1}-r}(\Phi)\left(X_{u_{1}}\right) Q^{1 / 2} h\right] d r \\
& G_{2}:=\int_{0}^{u_{2}} e^{\left(t-u_{1}\right) A}\left[D \mathcal{R}_{u_{1}-r}(\Phi)\left(X_{u_{1}}\right) Q^{1 / 2} h\right] d r-\int_{0}^{u_{2}} e^{\left(t-u_{1}\right) A}\left[D \mathcal{R}_{u_{2}-r}(\Phi)\left(X_{u_{1}}\right) Q^{1 / 2} h\right] d r .
\end{aligned}
$$


We also see that

$$
\begin{aligned}
& \left\|e^{\left(t-u_{1}\right) A} \int_{0}^{u_{2}}\left(D \mathcal{R}_{u_{1}-r}(\Phi)\left(X_{u_{1}}\right)-D \mathcal{R}_{u_{2}-r}(\Phi)\left(X_{u_{1}}\right)\right) d r Q^{1 / 2}\right\|_{E}^{2} \\
= & \left\|e^{\left(t-u_{1}\right) A} \int_{0}^{u_{2}}\left(D \mathcal{R}_{u_{1}-r}(\Phi)\left(X_{u_{1}}\right)-D \mathcal{R}_{u_{2}-r}(\Phi)\left(X_{u_{1}}\right)\right) d r Q^{1 / 2} M\right\|_{H . S .}^{2} \\
= & \sum_{n, h \geq 1}\left\langle e^{\left(t-u_{1}\right) A} \int_{0}^{u_{2}}\left(D \mathcal{R}_{u_{1}-r}(\Phi)\left(X_{u_{1}}\right)-D \mathcal{R}_{u_{2}-r}(\Phi)\left(X_{u_{1}}\right)\right) d r Q^{1 / 2} M e_{h}, e_{n}\right\rangle^{2} \\
= & \sum_{n, h \geq 1} e^{-2 \alpha_{n}\left(t-u_{1}\right)}\left\langle\int_{0}^{u_{2}}\left(D \mathcal{R}_{u_{1}-r}(\Phi)\left(X_{u_{1}}\right)-D \mathcal{R}_{u_{2}-r}(\Phi)\left(X_{u_{1}}\right)\right) d r Q^{1 / 2} M e_{h}, e_{n}\right\rangle^{2} \\
= & \sum_{n, h \geq 1} e^{-2 \alpha_{n}\left(t-u_{1}\right)}\left\langle\int_{0}^{u_{2}}\left(D R_{u_{1}-r}\left(\Phi_{n}\right)\left(X_{u_{1}}\right)-D R_{u_{2}-r}\left(\Phi_{n}\right)\left(X_{u_{1}}\right)\right) d r, Q^{1 / 2} M e_{h}\right\rangle^{2} \\
= & \sum_{n \geq 1} e^{-2 \alpha_{n}\left(t-u_{1}\right)} \sum_{h \geq 1}\left\langle Q^{1 / 2} M \int_{0}^{u_{2}}\left(D R_{u_{1}-r}\left(\Phi_{n}\right)\left(X_{u_{1}}\right)-D R_{u_{2}-r}\left(\Phi_{n}\right)\left(X_{u_{1}}\right)\right) d r, e_{h}\right\rangle^{2} \\
\leq & \sum_{n \geq 1} e^{-2 \alpha_{n}\left(t-u_{1}\right)}\left\|Q^{1 / 2} M\right\|^{2}\left\|\int_{0}^{u_{2}}\left(D R_{u_{1}-r}\left(\Phi_{n}\right)\left(X_{u_{1}}\right)-D R_{u_{2}-r}\left(\Phi_{n}\right)\left(X_{u_{1}}\right)\right) d r\right\|^{2} \\
= & \left\|Q^{1 / 2} M\right\|^{2} \sum_{n \geq 1}\left(2 \alpha_{n}\left(t-u_{1}\right)\right)^{1-\delta} e^{-2 \alpha_{n}\left(t-u_{1}\right)}\left(2 \alpha_{n}\left(t-u_{1}\right)\right)^{-(1-\delta)} . \\
& \cdot\left\|\int_{0}^{u_{2}}\left(D R_{u_{1}-r}\left(\Phi_{n}\right)\left(X_{u_{1}}\right)-D R_{u_{2}-r}\left(\Phi_{n}\right)\left(X_{u_{1}}\right)\right) d r\right\| \\
\leq & C_{\delta}\left\|Q^{1 / 2} M\right\|^{2}\left\|\int_{0}^{u_{2}}\left(D \mathcal{R}_{u_{1}-r}(\Phi)\left(X_{u_{1}}\right)-D \mathcal{R}_{u_{2}-r}(\Phi)\left(X_{u_{1}}\right)\right) d r\right\|^{2} 2^{-(1-\delta)} \frac{1}{\left(t-u_{1}\right)^{1-\delta}} .
\end{aligned}
$$

On the other hand it follows from the semigroup property of $\mathcal{R}_{t}$ that

$$
D \mathcal{R}_{u_{1}-r}(\Phi)(x)=D\left(\mathcal{R}_{u_{2}-r}\left(\mathcal{R}_{u_{1}-u_{2}} \Phi\right)\right)(x) .
$$

This in connection with Theorem 2 gives

$$
\begin{aligned}
& \int_{0}^{u_{2}}\left(D \mathcal{R}_{u_{1}-r}(\Phi)\left(X_{u_{1}}\right)-D \mathcal{R}_{u_{2}-r}(\Phi)\left(X_{u_{1}}\right)\right) h d r \\
& \int_{0}^{u_{2}} \int_{H}\left\langle\Lambda_{u_{2}-r} h, Q_{u_{2}-r}^{-1 / 2} y\right\rangle\left(\left(\mathcal{R}_{u_{1}-u_{2}} \Phi-\mathcal{R}_{0} \Phi\right)\left(e^{\left(u_{2}-r\right) A} X_{u_{1}}+y\right)\right) N_{Q_{u_{2}-r}}(d y) d r .
\end{aligned}
$$

Further, we find for $a=e^{\left(u_{2}-r\right) A} X_{u_{1}}+y$ and arbitrarly small $0<2 \rho<1$ by using Burkholder's 
inequality, Lemma 5.8 and (5.21)

$$
\begin{aligned}
& \left\|\left(\mathcal{R}_{u_{1}-u_{2}} \Phi-\mathcal{R}_{0} \Phi\right)(a)\right\|^{2} \\
= & \left\|E\left[\Phi\left(r, Z_{u_{1}-u_{2}}^{a}\right)-\Phi(r, a)\right]\right\|^{2} \\
\leq & \|\Phi\|_{\alpha}^{2} E\left[\left\|Z_{u_{1}-u_{2}}^{a}-Z_{0}^{a}\right\|^{2 \alpha}\right] \\
= & \|\Phi\|_{\alpha}^{2} E\left[\|\left(e^{\left(u_{1}-u_{2}\right) A} a-a+\int_{0}^{u_{1}-u_{2}} e^{\left(u_{1}-u_{2}-s\right) A} Q^{1 / 2} d W_{s} \|^{2 \alpha}\right]\right. \\
\leq & C\|\Phi\|_{\alpha}^{2}\left(\left\|e^{\left(u_{1}-r\right) A} X_{u_{1}}\left(\omega_{2}\right)-e^{\left(u_{2}-r\right) A} X_{u_{1}}\left(\omega_{2}\right)\right\|^{2 \alpha}\right. \\
& \left.+\left\|e^{\left(u_{1}-u_{2}\right) A} y-y\right\|^{2 \alpha}+E\left[\left\|\int_{0}^{u_{1}-u_{2}} e^{\left(u_{1}-u_{2}-s\right) A} Q^{1 / 2} d W_{s}\right\|^{2 \alpha}\right]\right) \\
\leq & C_{\alpha}\|\Phi\|_{\alpha}^{2}\left(\left\|e^{\left(u_{1}-r\right) A} A^{-\rho}\left(A^{\rho} X_{u_{1}}\left(\omega_{2}\right)\right)-e^{\left(u_{2}-r\right) A} A^{-\rho}\left(A^{\rho} X_{u_{1}}\left(\omega_{2}\right)\right)\right\|^{2 \alpha}\right. \\
& \left.+\left\|e^{\left(u_{1}-u_{2}\right) A} y-y\right\|^{2 \alpha}+\int_{0}^{u_{1}-u_{2}}\left\|e^{\left(u_{1}-u_{2}-s\right) A} Q^{1 / 2}\right\|_{H . S .}^{2 \alpha} d s\right) \\
\leq & C_{\alpha}\|\Phi\|_{\alpha}^{2}\left(\left|u_{1}-u_{2}\right|^{2 \rho \alpha}\left\|A^{\rho} X_{u_{1}}\left(\omega_{2}\right)\right\|^{2 \alpha}+\left\|e^{\left(u_{1}-u_{2}\right) A} y-y\right\|^{2 \alpha}\right. \\
& \left.+\frac{1}{1-(1-\delta) \alpha}\left(u_{1}-u_{2}\right)^{1-(1-\delta) \alpha}\right) .
\end{aligned}
$$

Hence by (5.20) in connection with Hölder's in inquality we get

$$
\begin{aligned}
& \left\|\int_{0}^{u_{2}}\left(D \mathcal{R}_{u_{1}-r}(\Phi)\left(X_{u_{1}}\right)-D \mathcal{R}_{u_{2}-r}(\Phi)\left(X_{u_{1}}\right)\right) d r\right\|^{2} \\
\leq & \left(\int_{0}^{u_{2}}\left\|\Lambda_{u_{2}-r}\right\|\left(\int_{H}\left\|\left(\left(\mathcal{R}_{u_{1}-u_{2}} \Phi-\mathcal{R}_{0} \Phi\right)\left(e^{\left(u_{2}-r\right) A} X_{u_{1}}+y\right)\right)\right\|^{2} N_{Q_{u_{2}-r}}(d y)\right)^{1 / 2} d r\right)^{2} \\
\leq & \left(\int _ { 0 } ^ { u _ { 2 } } \| \Lambda _ { u _ { 2 } - r } \| \left(C _ { \alpha } \| \Phi \| _ { \alpha } ^ { 2 } \left(\left|u_{1}-u_{2}\right|^{2 \rho \alpha}\left\|A^{\rho} X_{u_{1}}\left(\omega_{2}\right)\right\|^{2 \alpha}\right.\right.\right. \\
& \left.\left.+\int_{H}\left\|e^{\left(u_{1}-u_{2}\right) A} y-y\right\|^{2 \alpha} N_{Q_{u_{2}-r}}(d y) d r+\frac{1}{1-(1-\delta) \alpha}\left(u_{1}-u_{2}\right)^{1-(1-\delta) \alpha}\right)^{1 / 2} d r\right)^{2} \\
\leq & C_{\alpha}\left(\int_{0}^{u_{2}}\left\|\Lambda_{u_{2}-r}\right\| d r\right)^{2}\left(C _ { \alpha } \| \Phi \| _ { \alpha } ^ { 2 } \left(\left|u_{1}-u_{2}\right|^{2 \rho \alpha}\left\|A^{\rho} X_{u_{1}}\left(\omega_{2}\right)\right\|^{2 \alpha}\right.\right. \\
& \left.+\left|u_{1}-u_{2}\right|^{\eta(\alpha /(1-\alpha))}+\frac{1}{1-(1-\delta) \alpha}\left(u_{1}-u_{2}\right)^{1-(1-\delta) \alpha}\right)
\end{aligned}
$$

So for $T<1$ we have

$$
\begin{aligned}
& E\left[\left\|G_{2}\right\|_{E}^{2}\right] \\
\leq & C_{\delta, \alpha, \eta, \rho, T}\left\|Q^{1 / 2} M\right\|^{2}\left(\int_{0}^{T}\left\|\Lambda_{u_{2}-r}\right\| d r\right)^{2}\left(\|\Phi\|_{\alpha}^{2} E\left[\left\|A^{\rho} X_{u_{1}}\right\|^{2 \alpha}\right]+1\right)^{2} \\
& \cdot \frac{1}{\left(t-u_{1}\right)^{1-\delta}}\left|u_{1}-u_{2}\right|^{(2 \rho \alpha) \wedge \eta(\alpha /(1-\alpha)) \wedge(1-(1-\delta) \alpha)} \\
\leq & C_{\delta, \alpha, \eta, \rho, T}\left\|Q^{1 / 2} M\right\|^{2}\left(\int_{0}^{T}\left\|\Lambda_{u_{2}-r}\right\| d r\right)^{2}\left(\left(C_{1}\|B\|_{\alpha}^{3}+C_{2}\|B\|_{\alpha}^{2}\right) E\left[\left\|A^{\rho} X_{u_{1}}\right\|^{2 \alpha}\right]+1\right)^{2} \\
& \cdot \frac{1}{\left(t-u_{1}\right)^{1-\delta}}\left|u_{1}-u_{2}\right|^{(2 \rho \alpha) \wedge \eta(\alpha /(1-\alpha)) \wedge(1-(1-\delta) \alpha)}
\end{aligned}
$$


On the other hand we know by Lemma 5.8 in connection with Hölder's inequality that

$$
E\left[\left\|A^{\rho} X_{u_{1}}\right\|^{2 \alpha}\right] \leq C\left(1+\|B\|_{\alpha}^{2}\right)^{\alpha /(1-\alpha)} \frac{1}{u_{1}^{2 \rho(\alpha /(1-\alpha))}}
$$

for arbitrarly small $\rho>0$.

$$
\begin{aligned}
& E\left[\left\|G_{2}\right\|_{E}^{2}\right] \\
\leq & C_{\delta, \alpha, \eta, \rho, T}\left\|Q^{1 / 2} M\right\|^{2}\left(\int_{0}^{T}\left\|\Lambda_{u_{2}-r}\right\| d r\right)^{2}\left(\left(C_{1}\|B\|_{\alpha}^{3}+C_{2}\|B\|_{\alpha}^{2}\right)\left(1+\|B\|_{\alpha}^{2}\right)^{\alpha /(1-\alpha)}+1\right)^{2} \\
& \cdot \frac{1}{u_{1}^{2 \rho(\alpha /(1-\alpha))}} \frac{1}{\left(t-u_{1}\right)^{1-\delta}}\left|u_{1}-u_{2}\right|^{(2 \rho \alpha) \wedge \eta(\alpha /(1-\alpha)) \wedge(1-(1-\delta) \alpha)}
\end{aligned}
$$

for arbitrarly small $\rho>0$.

As for the term $G_{1}$ we argue just as above and get

$$
\begin{aligned}
& \left\|e^{\left(t-u_{1}\right) A} \int_{u_{2}}^{u_{1}} D \mathcal{R}_{u_{1}-r}(\Phi)\left(X_{u_{1}}\right) d r Q^{1 / 2}\right\|_{E}^{2} \\
\leq & C_{\delta}\left\|Q^{1 / 2} M\right\|^{2}\left\|\int_{u_{2}}^{u_{1}} D \mathcal{R}_{u_{1}-r}(\Phi)\left(X_{u_{1}}\right) d r\right\|^{2} 2^{-(1-\delta)} \frac{1}{\left(t-u_{1}\right)^{1-\delta}} .
\end{aligned}
$$

But

$$
\begin{aligned}
& \left\|\int_{u_{2}}^{u_{1}} D \mathcal{R}_{u_{1}-r}(\Phi)\left(X_{u_{1}}\right) d r\right\|^{2} \\
\leq & C\left(\int_{u_{2}}^{u_{1}}\left\|\Lambda_{u_{1}-r}\right\| d r\right)^{2}\|\Phi\|_{0}^{2} \\
\leq & C\left(\int_{0}^{u_{1}-u_{2}}\left\|\Lambda_{r}\right\| d r\right)^{2} C_{T}\left(K_{1}\|B\|_{\alpha}^{2}+K_{2}\|B\|_{\alpha}\right)^{2} \\
\leq & C\left(\int_{0}^{T}\left\|\Lambda_{u_{1}-r}\right\|^{1+\theta} d r\right)^{2 /(1+\theta)} C_{T}\left(K_{1}\|B\|_{\alpha}^{2}+K_{2}\|B\|_{\alpha}\right)^{2}\left(u_{1}-u_{2}\right)^{\frac{\theta}{1+\theta}}
\end{aligned}
$$

Hence

$$
\begin{aligned}
& E\left[\left\|G_{1}\right\|_{E}^{2}\right] \\
& C_{\delta}\left\|Q^{1 / 2} M\right\|^{2} 2^{-(1-\delta)} C\left(\int_{0}^{T}\left\|\Lambda_{u_{1}-r}\right\|^{1+\theta} d r\right)^{2 /(1+\theta)} C_{T}\left(K_{1}\|B\|_{\alpha}^{2}+K_{2}\|B\|_{\alpha}\right)^{2}\left(u_{1}-u_{2}\right)^{\frac{\theta}{1+\theta}} \frac{1}{\left(t-u_{1}\right)^{1-\delta}} .
\end{aligned}
$$

So we obtain

$$
\begin{aligned}
& E\left[\left\|T_{3}\right\|_{E}^{2}\right] \\
\leq & C_{\delta, \alpha, \eta, \rho, T}\left\|Q^{1 / 2} M\right\|^{2}\left(\int_{0}^{T}\left\|\Lambda_{u_{2}-r}\right\| d r\right)^{2}\left(\left(C_{1}\|B\|_{\alpha}^{3}+C_{2}\|B\|_{\alpha}^{2}\right)\left(1+\|B\|_{\alpha}^{2}\right)^{\alpha /(1-\alpha)}+1\right)^{2} \\
& \cdot \frac{1}{u_{1}^{2 \rho(\alpha /(1-\alpha))}} \frac{1}{\left(t-u_{1}\right)^{1-\delta}}\left|u_{1}-u_{2}\right|^{(2 \rho \alpha) \wedge \eta(\alpha /(1-\alpha)) \wedge(1-(1-\delta) \alpha)} \\
& +2 C_{\delta}\left\|Q^{1 / 2} M\right\|^{2} 2^{-(1-\delta)}\left(C_{1}\|B\|_{\alpha}^{3}+C_{2}\|B\|_{\alpha}^{2}\right)^{2} \sup _{0 \leq s \leq T}\left\|e^{s A} Q^{1 / 2}\right\|_{H S}^{2 \alpha}\left(u_{1}-u_{2}\right)^{2 \alpha} \frac{1}{\left(t-u_{1}\right)^{1-\delta}} .
\end{aligned}
$$


2.3. $T_{1}$ : We find that

$$
\begin{aligned}
& \left\|\left(e^{\left(t-u_{2}\right) A}-e^{\left(t-u_{1}\right) A}\right) D U\left(u_{2}, X_{u_{2}}\right) Q^{1 / 2}\right\|_{E}^{2} \\
= & \sum_{h \geq 1}\left\|\left(e^{\left(t-u_{2}\right) A}-e^{\left(t-u_{1}\right) A}\right) D U\left(u_{2}, X_{u_{2}}\right) Q^{1 / 2} M e_{h}\right\|^{2} \\
= & \sum_{n, h \geq 1}\left\langle\left(e^{\left(t-u_{2}\right) A}-e^{\left(t-u_{1}\right) A}\right) D U\left(u_{2}, X_{u_{2}}\right) Q^{1 / 2} M e_{h}, e_{n}\right\rangle^{2} \\
= & \sum_{n, h \geq 1}\left(e^{-\left(t-u_{2}\right) \alpha_{n}}-e^{-\left(t-u_{1}\right) \alpha_{n}}\right)^{2}\left\langle D U_{n}\left(u_{2}, X_{u_{2}}\right), Q^{1 / 2} M e_{h}\right\rangle^{2} \\
= & \sum_{n, h \geq 1}\left(e^{-\left(t-u_{2}\right) \alpha_{n}}-e^{-\left(t-u_{1}\right) \alpha_{n}}\right)^{2}\left\langle Q^{1 / 2} M D U_{n}\left(u_{2}, X_{u_{2}}\right), e_{h}\right\rangle^{2} \\
\leq & \left\|Q^{1 / 2} M\right\|^{2}\|D U\|_{0}^{2} \sum_{n \geq 1}\left(e^{-\left(t-u_{2}\right) \alpha_{n}}-e^{-\left(t-u_{1}\right) \alpha_{n}}\right)^{2} \\
= & \left\|Q^{1 / 2} M\right\|^{2}\|D U\|_{0}^{2} \sum_{n \geq 1} e^{-2\left(t-u_{1}\right) \alpha_{n}}\left(1-e^{-\left(u_{1}-u_{2}\right) \alpha_{n}}\right)^{2} \\
= & \left\|Q^{1 / 2} M\right\|^{2}\|D U\|_{0}^{2} \cdot \\
& \cdot \sum_{n \geq 1}\left(2\left(t-u_{1}\right) \alpha_{n}\right)^{1-\delta} e^{-2\left(t-u_{1}\right) \alpha_{n}}\left(2\left(t-u_{1}\right) \alpha_{n}\right)^{\delta-1}\left(1-e^{-\left(u_{1}-u_{2}\right) \alpha_{n}}\right)^{2} \\
= & \left\|Q^{1 / 2} M\right\|^{2}\|D U\|_{0}^{2} \cdot \\
& \cdot \sum_{n \geq 1}\left(2\left(t-u_{1}\right) \alpha_{n}\right)^{1-\delta} e^{-2\left(t-u_{1}\right) \alpha_{n}} 2^{\delta-1}\left(t-u_{1}\right)^{\delta-1}\left(u_{1}-u_{2}\right)^{1-\delta}\left(\left(u_{1}-u_{2}\right) \alpha_{n}\right)^{\delta-1}\left(1-e^{-\left(u_{1}-u_{2}\right) \alpha_{n}}\right)^{2} \\
\leq & C_{\delta}\left\|Q^{1 / 2} M\right\|^{2}\|D U\|_{0}^{2} \frac{1}{\left(t-u_{1}\right)^{1-\delta}}\left(u_{1}-u_{2}\right)^{1-\delta} \\
= & C_{T} C_{\delta}\left\|Q^{1 / 2} M\right\|^{2}\|B\|_{\alpha}^{2} \frac{1}{\left(t-u_{1}\right)^{1-\delta}}\left(u_{1}-u_{2}\right)^{1-\delta} \cdot \\
& \\
& \\
&
\end{aligned}
$$

Hence

$$
\begin{aligned}
& E\left[\left\|T_{1}\right\|_{E}^{2}\right] \\
\leq & C_{T} C_{\delta}\left\|Q^{1 / 2} M\right\|^{2}\|B\|_{\alpha}^{2} \frac{1}{\left(t-u_{1}\right)^{1-\delta}}\left(u_{1}-u_{2}\right)^{1-\delta} .
\end{aligned}
$$

Altogether we see for $T<1$ that

$$
\begin{aligned}
& E\left[\left\|J_{5}\right\|_{E}^{2}\right] \\
\leq & C(T, \delta, \alpha, M, Q, \theta, \mu, \vartheta, \varepsilon, A) G\left(\|B\|_{\alpha}\right) \\
& \cdot\left(\frac{1}{\left(t-u_{1}\right)^{1-\delta}}\left(u_{1}-u_{2}\right)^{1-\delta}+\frac{1}{\left(t-u_{1}\right)^{1-\delta}} \frac{1}{u_{2}^{2 \varepsilon}}\left(u_{1}-u_{2}\right)^{2 \varepsilon \wedge \delta \wedge 1}\right. \\
& \left.+\frac{1}{u_{1}^{2 \rho(\alpha /(1-\alpha))}} \frac{1}{\left(t-u_{1}\right)^{1-\delta}}\left|u_{1}-u_{2}\right|^{(2 \rho \alpha) \wedge \eta(\alpha /(1-\alpha)) \wedge(1-(1-\delta) \alpha \wedge 2 \alpha)}\right),
\end{aligned}
$$

where $G$ is a non-decreasing continuous function on $[0, \infty)$. 
3. Estimate for $J_{2}$ : The calculation in 2. for the estimate $I_{2}$ shows that

$$
\begin{aligned}
& E\left[\left\|J_{2}\right\|_{E}^{2}\right] \\
\leq & \left(u_{1}-u_{2}\right) C_{\varepsilon, T} C_{\gamma}\|B\|_{0}^{2} \int_{u_{2}}^{u_{1}} \frac{1}{\left(u_{1}-s\right)^{2(1-\gamma)}} E\left[\left\|D_{u_{2}} X_{s}\right\|_{E}^{2}\right] d s
\end{aligned}
$$

Then employing the estimate (5.29) we have

$$
\begin{aligned}
& E\left[\left\|J_{2}\right\|_{E}^{2}\right] \\
\leq \quad & \left(u_{1}-u_{2}\right) C C_{\varepsilon, T} C_{\gamma}\|B\|_{0}^{2} \\
& \cdot\left(G_{1}\left(\|B\|_{\alpha}\right) \int_{u_{2}}^{u_{1}} \frac{1}{\left(u_{1}-s\right)^{2(1-\gamma)}} \frac{1}{\left(s-u_{2}\right)^{1-\delta}} d s\right. \\
& \left.+G_{2}\left(\|B\|_{\alpha}\right) \int_{u_{2}}^{u_{1}} \frac{1}{\left(u_{1}-s\right)^{2(1-\gamma)}}\left(s-u_{2}\right)^{\delta} d s\right) \\
= & \left(u_{1}-u_{2}\right) C C_{\varepsilon, T} C_{\gamma}\|B\|_{0}^{2} \\
& \cdot\left(G_{1}\left(\|B\|_{\alpha}\right)\left(u_{1}-u_{2}\right)^{1-2(1-\gamma)-(1-\delta)} \frac{\Gamma(1-2(1-\gamma)) \Gamma(1-\delta)}{\Gamma(1-2(1-\gamma)+1-\delta)}\right. \\
& \left.+G_{2}\left(\|B\|_{\alpha}\right)\left(u_{1}-u_{2}\right)^{1-2(1-\gamma)+\delta} \frac{\Gamma(1-2(1-\gamma)) \Gamma(1+\delta)}{\Gamma(1-2(1-\gamma)+1+\delta)}\right) \\
= & C(\varepsilon, T, \gamma, \delta)\left(G_{1}\left(\|B\|_{\alpha}\right)\left(u_{1}-u_{2}\right)^{1-2(1-\gamma)+\delta}\right. \\
& \left.+G_{2}\left(\|B\|_{\alpha}\right)\left(u_{1}-u_{2}\right)^{1-2(1-\gamma)+\delta}\right),
\end{aligned}
$$

where we used the Beta function and where $0<2(1-\gamma)<1$ and $G_{i}, i=1,2$ are non-negative functions on an interval $[0, V(T)]$ with $V(T) \longrightarrow \infty$ for $T \longrightarrow 0$.

4. Estimate for $J_{6}$ : We argue just as in 5. for the estimate $I_{5}$ and get in connection with the inequality (5.29) for $T<1$ 


$$
\begin{aligned}
& \sum_{r \geq 1} \sum_{h \geq 1} \int_{u_{2}}^{u_{1}}\left\|e^{(t-s) A} D^{2} U\left(s, X_{s}\right)\left[Q^{1 / 2} e_{h}, D_{u_{2}} X_{s} M e_{r}\right]\right\|^{2} d s \\
= & \sum_{r \geq 1} \sum_{n, h \geq 1} \int_{u_{2}}^{u_{1}}\left\langle e^{(t-s) A} D^{2} U\left(s, X_{s}\right)\left[Q^{1 / 2} e_{h}, D_{u_{2}} X_{s} M e_{r}\right], e_{n}\right\rangle^{2} d s \\
= & \sum_{r \geq 1} \sum_{n, h \geq 1} \int_{u_{2}}^{u_{1}} e^{-2 \alpha_{n}(t-s)}\left\langle D^{2} U\left(s, X_{s}\right)\left[Q^{1 / 2} e_{h}, D_{u_{2}} X_{s} M e_{r}\right], e_{n}\right\rangle^{2} d s \\
= & \sum_{r \geq 1} \sum_{n, h \geq 1} \int_{u_{2}}^{u_{1}} e^{-2 \alpha_{n}(t-s)}\left(D^{2} U_{n}\left(s, X_{s}\right)\left[Q^{1 / 2} e_{h}, D_{u_{2}} X_{s} M e_{r}\right]\right)^{2} d s \\
= & \sum_{r \geq 1} \sum_{n \geq 1} \int_{u_{2}}^{u_{1}} e^{-2 \alpha_{n}(t-s)} \sum_{h \geq 1}\left(D^{2} U_{n}\left(s, X_{s}\right)\left[Q^{1 / 2} e_{h}, D_{u_{2}} X_{s} M e_{r}\right]\right)^{2} d s \\
\leq & \sum_{n \geq 1} \int_{u_{2}}^{u_{1}} e^{-2 \alpha_{n}(t-s)}\|Q\|\left\|D^{2} U_{n}\right\|_{0}^{2}\left\|D_{u_{2}} X_{s}\right\|_{E}^{2} d s \\
\leq & C_{T} \sum_{n \geq 1} \int_{u_{2}}^{u_{1}} e^{-2 \alpha_{n}(t-s)}\|Q\|\|B\|_{\alpha}^{2}\left\|D_{u_{2}} X_{s}\right\|_{E}^{2} d s \\
\leq & C_{T} F\left(\|B\|_{\alpha}\right)\|B\|_{\alpha}^{2} \sum_{n \geq 1} \int_{u_{2}}^{u_{1}} e^{-2 \alpha_{n}(t-s)} \frac{1}{\left(s-u_{2}\right)^{1-\delta}} d s,
\end{aligned}
$$

where $F$ is a non-decreasing continuous function on $[0, \infty)$. 
On the other hand we see by integration by parts and Hölder's inequality that

$$
\begin{aligned}
& \sum_{n \geq 1} \int_{u_{2}}^{u_{1}} e^{-2 \alpha_{n}(t-s)} \frac{1}{\left(s-u_{2}\right)^{1-\delta}} d s \\
& =\sum_{n \geq 1}\left(\frac{1}{\delta} e^{-2 \alpha_{n}\left(t-u_{1}\right)}\left(u_{1}-u_{2}\right)^{\delta}-2 \alpha_{n} \int_{u_{2}}^{u_{1}} e^{-2 \alpha_{n}(t-s)} \frac{1}{\delta}\left(s-u_{2}\right)^{\delta} d s\right) \\
& \leq \sum_{n \geq 1}\left(\frac{1}{\delta} e^{-2 \alpha_{n}\left(t-u_{1}\right)}\left(u_{1}-u_{2}\right)^{\delta}+\frac{1}{\delta} 2 \alpha_{n}\left(\int_{u_{2}}^{u_{1}} e^{-2 \alpha_{n}(1+\tau)(t-s)} d s\right)^{1 /(1+\tau)}\left(\int_{u_{2}}^{u_{1}}\left(s-u_{2}\right)^{\delta\left(1+\tau^{-1}\right)} d s\right)^{1 /\left(1+\tau^{-1}\right)}\right) \\
& =\sum_{n \geq 1}\left(\frac{1}{\delta} e^{-2 \alpha_{n}\left(t-u_{1}\right)}\left(u_{1}-u_{2}\right)^{\delta}+\frac{1}{\delta} 2 \alpha_{n}\left(\frac{1}{2(1+\tau) \alpha_{n}}\left(e^{-2 \alpha_{n}(1+\tau)\left(t-u_{1}\right)}-e^{-2 \alpha_{n}(1+\tau)\left(t-u_{2}\right)}\right)\right)^{1 /(1+\tau)}\right. \\
& \left.\cdot\left(\frac{1}{\left(1+\tau^{-1}\right) \delta+1}\right)^{1 /(1+\tau)}\left(u_{1}-u_{2}\right)^{\delta+\frac{1}{\left(1+\tau^{-1}\right)}}\right) \\
& =\sum_{n \geq 1}\left(\frac{1}{\delta}\left(2 \alpha_{n}\left(t-u_{1}\right)\right)^{1-\delta} e^{-2 \alpha_{n}\left(t-u_{1}\right)} \frac{1}{\left(2 \alpha_{n}\left(t-u_{1}\right)\right)^{1-\delta}}\left(u_{1}-u_{2}\right)^{\delta}\right) \\
& +\frac{1}{\delta}\left(\frac{1}{2(1+\tau)}\right)^{1 /(1+\tau)}\left(\frac{1}{\left(1+\tau^{-1}\right) \delta+1}\right)^{1 /(1+\tau)} \\
& \cdot \sum_{n \geq 1} \frac{1}{\alpha_{n}^{\frac{1}{1+\tau}-\delta}}\left(2 \alpha_{n}\left(t-u_{1}\right)\right)^{1-\delta} e^{-2 \alpha_{n}\left(t-u_{1}\right)} \frac{1}{\left(t-u_{1}\right)^{1-\delta}}\left(1-e^{-2 \alpha_{n}(1+\tau)\left(u_{1}-u_{2}\right)}\right)^{1 /(1+\tau)}\left(u_{1}-u_{2}\right)^{\delta+\frac{1}{(1+\tau-1)}} \\
& \leq C\left(\frac{1}{\left(t-u_{1}\right)^{1-\delta}}\left(u_{1}-u_{2}\right)^{\delta}+\frac{1}{\left(t-u_{1}\right)^{1-\delta}}\left(u_{1}-u_{2}\right)^{\delta+\frac{1}{\left(1+\tau^{-1}\right)}}\right) \\
& \leq C \frac{1}{\left(t-u_{1}\right)^{1-\delta}}\left(u_{1}-u_{2}\right)^{\delta}
\end{aligned}
$$

for $\tau>0$ small enough.

So we get

$$
\begin{aligned}
& E\left[\left\|J_{6}\right\|_{E}^{2}\right] \\
\leq & C_{T} F\left(\|B\|_{\alpha}\right)\|B\|_{\alpha}^{2} \frac{1}{\left(t-u_{1}\right)^{1-\delta}}\left(u_{1}-u_{2}\right)^{\delta}
\end{aligned}
$$

Let us now consider the terms $J_{1}, J_{3}$ and $J_{7}$. But this case just corresponds to the calculations for the estimates of $I_{1}, I_{2}$ and $I_{5}$ and we obtain

$$
\begin{aligned}
& E\left[\left\|J_{1}\right\|_{E}^{2}\right]+E\left[\left\|J_{3}\right\|_{E}^{2}\right]+E\left[\left\|J_{7}\right\|_{E}^{2}\right] \\
\leq & K_{T}\|B\|_{0}^{2} E\left[\left\|D_{u_{1}} X_{t}-D_{u_{2}} X_{t}\right\|\right]_{E}^{2} \\
& +\left(t-u_{1}\right) C_{\varepsilon, T}\|B\|_{0}^{2} E\left[\int_{u_{1}}^{t} \frac{C_{\gamma}}{(t-s)^{2(1-\gamma)}}\left\|D_{u_{1}} X_{s}-D_{u_{2}} X_{s}\right\|_{E}^{2} d s\right] \\
& +C_{\delta} C_{T}\|Q\| 2^{-(1-\delta)}\|B\|_{\alpha}^{2} \int_{u_{1}}^{t} \frac{1}{(t-s)^{1-\delta}}\left\|D_{u_{1}} X_{s}-D_{u_{2}} X_{s}\right\|_{E}^{2} d s
\end{aligned}
$$

Altogether it follows from the above estimates that 


$$
\begin{aligned}
& E\left[\left\|D_{u_{1}} X_{t}-D_{u_{2}} X_{t}\right\|_{E}^{2}\right] \\
\leq & \left.C\left(\sum_{i=1}^{7} E\left[\left\|J_{i}\right\|_{E}^{2}\right]\right)\right) \\
\leq & C\left(K_{T}\|B\|_{\alpha}^{2} E\left[\left\|D_{u_{1}} X_{t}-D_{u_{2}} X_{t}\right\|\right]_{E}^{2}\right. \\
& +C(\varepsilon, T, \gamma, \delta)\left(G_{1}\left(\|B\|_{\alpha}\right)\left(u_{1}-u_{2}\right)^{1-2(1-\gamma)+\delta}+G_{2}\left(\|B\|_{\alpha}\right)\left(u_{1}-u_{2}\right)^{1-2(1-\gamma)+\delta}\right) \\
& +C \frac{1}{\left(t-u_{1}\right)^{1-\delta}}\left|u_{1}-u_{2}\right|^{\mu} \\
& +C(T, \delta, \alpha, M, Q, \theta, \mu, \vartheta, \varepsilon, A) G\left(\|B\|_{\alpha}\right) \\
& +\left(\frac{1}{\left(t-u_{1}\right)^{1-\delta}}\left(u_{1}-u_{2}\right)^{1-\delta}+\frac{1}{\left(t-u_{1}\right)^{1-\delta}} \frac{1}{u_{2}^{2 \varepsilon}}\left(u_{1}-u_{2}\right)^{2 \varepsilon \wedge \delta \wedge 1}\right. \\
& \left.+\frac{1}{u_{1}^{2 \rho(\alpha /(1-\alpha))}} \frac{1}{\left(t-u_{1}\right)^{1-\delta}}\left|u_{1}-u_{2}\right|^{(2 \rho \alpha) \wedge \eta(\alpha /(1-\alpha)) \wedge(1-(1-\delta) \alpha \wedge 2 \alpha)}\right) \\
& +C_{T} F\left(\|B\|_{\alpha}\right)\|B\|_{\alpha}^{2} \frac{1}{\left(t-u_{1}\right)^{1-\delta}\left(u_{1}-u_{2}\right)^{\delta}} \\
& +\left(t-u_{1}\right) C_{\varepsilon, T}\|B\|_{0}^{2} E\left[\int_{u_{1}}^{t} \frac{C_{\gamma}}{(t-s)^{2(1-\gamma)}}\left\|D_{u_{1}} X_{s}-D_{u_{2}} X_{s}\right\|_{E}^{2} d s\right] \\
& \left.+C_{\delta} C_{T}\|Q\| 2^{-(1-\delta)}\|B\|_{\alpha}^{2} \int_{u_{1}}^{t} \frac{1}{(t-s)^{1-\delta}}\left\|D_{u_{1}} X_{s}-D_{u_{2}} X_{s}\right\|_{E}^{2} d s\right) .
\end{aligned}
$$

Since $K_{T} \longrightarrow 0$ for $T \longrightarrow 0$ and $1>T>u_{1}>u_{2}$ we get

$$
\begin{aligned}
& E\left[\left\|D_{u_{1}} X_{t}-D_{u_{2}} X_{t}\right\|_{E}^{2}\right] \\
\leq & V\left(\|B\|_{\alpha}\right) \frac{1}{\left(t-u_{1}\right)^{1-\delta}} \frac{1}{u_{2}^{2 \varepsilon \vee 2 \rho(\alpha /(1-\alpha))}}\left(u_{1}-u_{2}\right)^{\lambda} \\
& +V\left(\|B\|_{\alpha}\right) \int_{u_{1}}^{t} \frac{1}{(t-s)^{1-\delta}}\left\|D_{u_{1}} X_{s}-D_{u_{2}} X_{s}\right\|_{E}^{2} d s,
\end{aligned}
$$

where $\lambda=(1-2(1-\gamma)+\delta) \wedge \mu \wedge(1-\delta) \wedge(2 \varepsilon \wedge \delta \wedge 1) \wedge((2 \rho \alpha) \wedge \eta(\alpha /(1-\alpha)) \wedge(1-(1-\delta) \alpha \wedge 2 \alpha)) \wedge \delta>$ 0 and where $V$ is a non-negative continuous function on $\left[0, \frac{1}{A_{T}}\right]$ for $A_{T} \longrightarrow 0$ for $T \longrightarrow 0$.

So by a Lemma of Gronwall for weakly singular kernels (see [1, Theorem 3]) we get

$$
E\left[\left\|D_{u_{1}} X_{t}-D_{u_{2}} X_{t}\right\|_{E}^{2}\right] \leq a(t)+\int_{u_{1}}^{t} \sum_{n \geq 1} \frac{(g(t) \Gamma(\delta))^{n}}{\Gamma(n \delta)}(t-s)^{n \delta-1} a(s) d s
$$

for $u_{1} \leq t \leq T$, where

$$
a(t):=V\left(\|B\|_{\alpha}\right) \frac{1}{\left(t-u_{1}\right)^{1-\delta}} \frac{1}{u_{2}^{2 \varepsilon \vee 2 \rho(\alpha /(1-\alpha))}}\left(u_{1}-u_{2}\right)^{\lambda}
$$

and

$$
g(t) \equiv V\left(\|B\|_{\alpha}\right)
$$


Therefore we have by means of the Beta function

$$
\begin{aligned}
& E\left[\left\|D_{u_{1}} X_{t}-D_{u_{2}} X_{t}\right\|_{E}^{2}\right] \\
\leq & V\left(\|B\|_{\alpha}\right) \frac{1}{\left(t-u_{1}\right)^{1-\delta}} \frac{1}{u_{2}^{2 \varepsilon \vee 2 \rho(\alpha /(1-\alpha))}}\left(u_{1}-u_{2}\right)^{\lambda} \\
& +\sum_{n=1}^{n_{0}-1} \frac{\left(V\left(\|B\|_{\alpha}\right) \Gamma(\delta)\right)^{n} \Gamma(\delta)}{\Gamma((n+1) \delta)} \frac{1}{\left(t-u_{1}\right)^{1-(n+1) \delta}} \frac{1}{u_{2}^{2 \varepsilon \vee 2 \rho(\alpha /(1-\alpha))}}\left(u_{1}-u_{2}\right)^{\lambda} \\
& +\sum_{n \geq n_{0}} \frac{\left(V\left(\|B\|_{\alpha}\right) \Gamma(\delta)\right)^{n} \Gamma(\delta)}{\Gamma((n+1) \delta)}\left(t-u_{1}\right)^{(n+1) \delta-1} \frac{1}{u_{2}^{2 \varepsilon \vee 2 \rho(\alpha /(1-\alpha))}}\left(u_{1}-u_{2}\right)^{\lambda} \\
\leq & E\left(\|B\|_{\alpha}\right) \frac{1}{\left(t-u_{1}\right)^{1-\delta}} \frac{1}{u_{2}^{2 \varepsilon \vee 2 \rho(\alpha /(1-\alpha))}}\left(u_{1}-u_{2}\right)^{\lambda},
\end{aligned}
$$

where $V$ is a non-negative continuous function on $\left[0, \frac{1}{A_{T}}\right]$ for $A_{T} \longrightarrow 0$ for $T \longrightarrow 0$ and where $n_{0} \delta<1$, but $\left(n_{0}+1\right) \delta \geq 1$ for $n_{0} \in \mathbb{N}$.

Thus it follows for all $0 \leq u_{1}, u_{2}<t$ that

$$
\begin{aligned}
& E\left[\left\|D_{u_{1}} X_{t}-D_{u_{2}} X_{t}\right\|_{E}^{2}\right] \\
\leq & E\left(\|B\|_{\alpha}\right)\left(\frac{1}{\left(t-u_{1}\right)^{1-\delta}} \frac{1}{u_{2}^{2 \varepsilon \vee 2 \rho(\alpha /(1-\alpha))}}\left|u_{1}-u_{2}\right|^{\lambda}\right. \\
& \left.+\frac{1}{\left(t-u_{2}\right)^{1-\delta}} \frac{1}{u_{1}^{2 \varepsilon \vee 2 \rho(\alpha /(1-\alpha))}}\left|u_{1}-u_{2}\right|^{\lambda}\right)
\end{aligned}
$$


Now choose in (5.27) $\beta>0$ such that $\kappa:=1+2 \beta-\lambda<1$. Then

$$
\begin{aligned}
& \int_{0}^{t} \int_{0}^{t} \frac{1}{\left(t-u_{1}\right)^{1-\delta}} \frac{1}{u_{2}^{2 \varepsilon \vee 2 \rho(\alpha /(1-\alpha))}} \frac{1}{\left|u_{1}-u_{2}\right|^{\kappa}} d u_{1} d u_{2} \\
& =\int_{0}^{t} \int_{u_{2}}^{t} \frac{1}{\left(t-u_{1}\right)^{1-\delta}} \frac{1}{u_{2}^{2 \varepsilon \vee 2 \rho(\alpha /(1-\alpha))}} \frac{1}{\left|u_{1}-u_{2}\right|^{\kappa}} d u_{1} d u_{2} \\
& +\int_{0}^{t} \int_{0}^{u_{2}} \frac{1}{\left(t-u_{1}\right)^{1-\delta}} \frac{1}{u_{2}^{2 \varepsilon \vee 2 \rho(\alpha /(1-\alpha))}} \frac{1}{\left|u_{1}-u_{2}\right|^{\kappa}} d u_{1} d u_{2} \\
& \leq \int_{0}^{t} \frac{1}{u_{2}^{2 \varepsilon \vee 2 \rho(\alpha /(1-\alpha))}} \int_{u_{2}}^{t} \frac{1}{\left(t-u_{1}\right)^{1-\delta}} \frac{1}{\left|u_{1}-u_{2}\right|^{\kappa}} d u_{1} d u_{2} \\
& +\int_{0}^{t} \frac{1}{\left(t-u_{2}\right)^{1-\delta}} \int_{0}^{u_{2}} \frac{1}{u_{2}^{2 \varepsilon \vee 2 \rho(\alpha /(1-\alpha))}} \frac{1}{\left|u_{1}-u_{2}\right|^{\kappa}} d u_{1} d u_{2} \\
& =\frac{\Gamma(\delta) \Gamma(1-\kappa)}{\Gamma(\delta+1-\kappa)} \int_{0}^{t} \frac{1}{u_{2}^{2 \varepsilon \vee 2 \rho(\alpha /(1-\alpha))}} \frac{1}{\left(t-u_{2}\right)^{\kappa-\delta}} d u_{2} \\
& +\frac{\Gamma((1-2 \varepsilon) \vee 2 \rho(\alpha /(1-\alpha))) \Gamma(1-\kappa+\delta)}{\Gamma(1-2 \varepsilon \vee 2 \rho(\alpha /(1-\alpha))+1-\kappa+\delta)} \\
& \cdot \int_{0}^{t} \frac{1}{\left(t-u_{2}\right)^{1-\delta}} \frac{1}{u_{2}^{2 \varepsilon \vee 2 \rho(\alpha /(1-\alpha))+2 \beta-\lambda-\delta}} d u_{2} \\
& =\frac{\Gamma(\delta) \Gamma(1-\kappa)}{\Gamma(\delta+1-\kappa)} \frac{\Gamma(1-2 \varepsilon \vee 2 \rho(\alpha /(1-\alpha))) \Gamma(1-\kappa+\delta)}{\Gamma(1-2 \varepsilon \vee 2 \rho(\alpha /(1-\alpha))+1-\kappa+\delta))} \\
& \cdot \frac{1}{t^{2 \varepsilon \vee 2 \rho(\alpha /(1-\alpha))+2 \beta-\lambda-\delta}} \\
& +\frac{\Gamma(1-2 \varepsilon \vee 2 \rho(\alpha /(1-\alpha))) \Gamma(1-\kappa+\delta)}{\Gamma(1-2 \varepsilon \vee 2 \rho(\alpha /(1-\alpha))+1-\kappa+\delta)} \\
& \cdot \frac{\Gamma(\delta) \Gamma(1-(2 \varepsilon \vee 2 \rho(\alpha /(1-\alpha))+2 \beta-\lambda-\delta))}{\Gamma(\delta+1-(2 \varepsilon \vee 2 \rho(\alpha /(1-\alpha))+2 \beta-\lambda-\delta))} \frac{1}{t^{2 \varepsilon \vee 2 \rho(\alpha /(1-\alpha))+2 \beta-\lambda-2 \delta}} \\
& <\infty \text {, }
\end{aligned}
$$

since $2 \beta-\lambda<0$ and since $\varepsilon>$ and $\rho>0$ can be chosen arbitrarly small.

So we get the estimate (5.27), which completes the proof.

Let now $B^{n} \in C\left([0, T] ; C_{b}^{1}(H, H)\right) \cap C\left([0, T] ; C_{b}^{\alpha}(H, H)\right), n \geq 1$ be a sequence of functions and $B \in C\left([0, T] ; C_{b}^{\alpha}(H, H)\right)$ such that

$$
B^{n}(t, x) \longrightarrow B(t, x)
$$

for $n \longrightarrow \infty$ in $H$ for all $x$ and such that

$$
\left\|B^{n}\right\|_{\alpha} \leq K
$$

for a constant $K$ independent of $n$. See e.g. [11].

We also need the following Lemma:

Lemma 5.10 Suppose that $X_{t}^{n}, 0 \leq t \leq T, n \geq 1$ are the unique mild solutions to (5.1) with respect to the coefficients $B^{n}$ in (5.31) and (5.32). Let $X_{t}^{n, i}=\left\langle e_{i}, X_{t}^{n}\right\rangle$. Then there exists for all $i$ a subsequence $\left(n_{k}^{i}\right)_{k \geq 1}$ which only depends on (a sufficiently small) $T$ and $i$ such that for all $0 \leq t \leq T X_{t}^{n_{k}^{i}, i}$ converges in $L^{2}(\Omega)$ for $k \longrightarrow \infty$. 
Proof. We know that

$$
X_{t}^{n}=e^{t A} x+\int_{0}^{t} e^{(t-s) A} B^{n}\left(s, X_{s}\right) d s+\int_{0}^{t} e^{(t-s) A} Q^{1 / 2} d W_{s}, 0 \leq t \leq T .
$$

So

$$
X_{t}^{n, i}=\left\langle e_{i}, e^{t A} x\right\rangle+\int_{0}^{t}\left\langle e_{i}, e^{(t-s) A} B^{n}\left(s, X_{s}\right)\right\rangle d s+\int_{0}^{t}\left\langle e_{i}, e^{(t-s) A} Q^{1 / 2}, d W_{s}\right\rangle, 0 \leq t \leq T .
$$

Hence

$$
\begin{aligned}
& X_{t_{1}}^{n, i}-X_{t_{2}}^{n, i} \\
= & \left\langle e_{i}, e^{t_{1} A} x-e^{t_{2} A} x\right\rangle+\int_{0}^{t_{2}}\left\langle e_{i},\left(e^{\left(t_{1}-s\right) A}-e^{\left(t_{2}-s\right) A}\right) B^{n}\left(s, X_{s}^{n}\right)\right\rangle d s \\
& +\int_{t_{2}}^{t_{1}}\left\langle e_{i}, e^{\left(t_{1}-s\right) A} B^{n}\left(s, X_{s}^{n}\right)\right\rangle d s+\int_{0}^{t_{2}}\left\langle e_{i}, e^{\left(t_{1}-s\right) A}-e^{\left(t_{2}-s\right) A} Q^{1 / 2} d W_{s}\right\rangle \\
& +\int_{t_{2}}^{t_{1}}\left\langle e_{i}, e^{\left(t_{1}-s\right) A} Q^{1 / 2} d W_{s}\right\rangle
\end{aligned}
$$

for all $0 \leq t_{2} \leq t_{1} \leq T$.

Now let $f$ be an element of the Hida test function space $(\mathcal{S}) \subset L^{2}(\Omega)$. Denote by $(\mathcal{S})^{*}$ its topological dual (Hida distribution space). See [10] for further information on these spaces. Then $\left\langle\left(X_{t_{1}}^{n, i}-X_{t_{2}}^{n, i}\right), f\right\rangle_{(\mathcal{S})^{*},(\mathcal{S})}=E\left[\left(X_{t_{1}}^{n, i}-X_{t_{2}}^{n, i}\right) f\right]$, where $\langle\cdot, \cdot\rangle_{(\mathcal{S})^{*},(\mathcal{S})}$ is the dual pairing. So using (5.33) we get

$$
\begin{aligned}
& E\left[\left(X_{t_{1}}^{n, i}-X_{t_{2}}^{n, i}\right) f\right] \\
= & \left\langle e_{i}, e^{t_{1} A} x-e^{t_{2} A} x\right\rangle E[f]+\int_{0}^{t_{2}} E\left[\left\langle e_{i},\left(e^{\left(t_{1}-s\right) A}-e^{\left(t_{2}-s\right) A}\right) B^{n}\left(s, X_{s}^{n}\right)\right\rangle, f\right] d s \\
& +\int_{t_{2}}^{t_{1}} E\left[\left\langle e_{i}, e^{\left(t_{1}-s\right) A} B^{n}\left(s, X_{s}^{n}\right)\right\rangle, f\right] d s+E\left[\int_{0}^{t_{2}}\left\langle e_{i}, e^{\left(t_{1}-s\right) A}-e^{\left(t_{2}-s\right) A} Q^{1 / 2} d W_{s}, f\right]\right\rangle \\
& +E\left[\int_{t_{2}}^{t_{1}}\left\langle e_{i}, e^{\left(t_{1}-s\right) A} Q^{1 / 2} d W_{s}, f\right] .\right\rangle
\end{aligned}
$$

Thus it follows from (5.21) and (5.22)

$$
\begin{aligned}
& \left|E\left[\left(X_{t_{1}}^{n, i}-X_{t_{2}}^{n, i}\right) f\right]\right| \\
\leq & \left|\left\langle e_{i}, x\right\rangle\right|\left|e^{-t_{1} \alpha_{i}}-e^{-t_{2} \alpha_{i}}\right||E[f]|+\int_{0}^{t_{2}} E\left[\left|\left\langle e_{i}, B^{n}\left(s, X_{s}^{n}\right)\right\rangle f\right|\right]\left|\left(e^{-\left(t_{1}-s\right) \alpha_{i}}-e^{\left(t_{2}-s\right) \alpha_{i}}\right)\right| d s \\
& +\int_{t_{2}}^{t_{1}} E\left[\left|\left\langle e_{i}, B^{n}\left(s, X_{s}^{n}\right)\right\rangle f\right|\right] e^{-\left(t_{1}-s\right) \alpha_{i}} d s+\left(\int_{0}^{t_{2}}\left\|e^{\left(t_{1}-s\right) A}-e^{\left(t_{2}-s\right) A} Q^{1 / 2}\right\|_{H . S .}^{2} d s\right)^{1 / 2}\left(E\left[f^{2}\right]\right)^{1 / 2} \\
& +\left(\int_{t_{2}}^{t_{1}}\left\|e^{\left(t_{1}-s\right) A} Q^{1 / 2}\right\|_{H . S .}^{2} d s\right)^{1 / 2}\left(E\left[f^{2}\right]\right)^{1 / 2} \\
\leq & C_{i, T}\left|t_{1}-t_{2}\right|\left\|B^{n}\right\| E[|f|]+C\left(\int_{0}^{t_{2}} \frac{1}{\left(t_{2}-s\right)^{1-\delta}}\left|t_{1}-t_{2}\right|^{\vartheta} d s\right)^{1 / 2}\left(E\left[f^{2}\right]\right)^{1 / 2} \\
& +C\left(\int_{t_{2}}^{t_{1}} \frac{1}{\left(t_{1}-s\right)^{1-\delta}} d s\right)^{1 / 2}\left(E\left[f^{2}\right]\right)^{1 / 2} \\
\leq & C(i, T, \vartheta, \delta, K, f)\left|t_{1}-t_{2}\right|^{\vartheta \wedge(\delta / 2)} .
\end{aligned}
$$


So

$$
\sup _{n \geq 1} m^{T}\left(\left\langle\left(X^{n, i}, f\right\rangle_{(\mathcal{S})^{*},(\mathcal{S})}, \delta\right) \longrightarrow 0 \text { for } \delta \searrow 0,\right.
$$

where $m^{T}$ is the modulus of continuity given by

$$
m^{T}(g, \delta):=\max _{\substack{|t-s| \leq \delta \\ 0 \leq t, s \leq T}}|g(t)-g(s)| .
$$

So $\left\langle\left(X^{n, i}, f\right\rangle_{(\mathcal{S})^{*},(\mathcal{S})}\right.$ is relatively compact in $C([0, T])$ for all $f \in(\mathcal{S})$. Since $(\mathcal{S})^{*}$ is the dual of a countably Hilbertian nuclear space $(\mathcal{S})$, we can apply a result of I. Mitoma [14] and find that there exists for all $i$ a subsequence $\left(n_{k}^{i}\right)_{k \geq 1}$ which only depends on (a sufficiently small) $T$ and $i$ such that $X^{n_{k}^{i}, i}$ converges in $C\left([0, T] ;(\mathcal{S})^{*}\right)$.

On the other hand it follows from Lemma 5.9 that there exists (for fixed $t$ ) a $C<\infty$ and a $0<\beta<\frac{1}{2}$ such that

$$
E\left[\int_{0}^{t}\left\|D_{u} X_{t}^{n}\right\|_{E}^{2} d u\right] \leq L_{1}\left(\left\|B^{n}\right\|_{\alpha}^{2}\right) \leq C<\infty
$$

and

$$
\begin{aligned}
& E\left[\int_{0}^{t} \int_{0}^{t} \frac{\left\|D_{u_{1}} X_{t}^{n}-D_{u_{2}} X_{t}^{n}\right\|_{E}^{2}}{\left|u_{1}-u_{2}\right|^{1+2 \beta}} d u_{1} d u_{2}\right] \\
\leq & L_{2}\left(\left\|B^{n}\right\|_{\alpha}^{2}\right) \leq C<\infty
\end{aligned}
$$

for all $n \geq 1$, provided that $T$ is sufficiently small.

Then, if we apply Theorem 5.14 in connection with Remark 5.15 in the Appendix to the sequence $X_{t}^{n_{k}^{i}, i}$ we see that for all $t$ and $i$ there exists a subsequence $m_{l}=m_{l}^{t, i}, l \geq 1$ of $n_{k}^{i}, k \geq 1$ and a $\widetilde{X}_{t}^{i} \in L^{2}(\Omega)$ such that

$$
X_{t}^{n_{m_{l}}^{i}, i} \longrightarrow \tilde{X}_{t}^{i} \text { for } l \longrightarrow \infty
$$

in $L^{2}(\Omega)$.

We claim that

$$
X_{t}^{n_{k}^{i}, i} \longrightarrow \widetilde{X}_{t}^{i} \text { for } k \longrightarrow \infty \text { in } L^{2}(\Omega)
$$

for all $t, i$. To see this assume that there exists for some $t, i$ a $\varepsilon>0$ and a subsequence $\varphi_{l}, l \geq 1$ such that

$$
\left\|X_{t}^{n_{\varphi_{l}}^{i}, i}-\widetilde{X}_{t}^{i}\right\|_{L^{2}(\Omega)} \geq \varepsilon
$$

On the other hand we know by Theorem 5.14 that there exists a subsequence $\phi_{r}, r \geq 1$ of such that

$$
X_{t}^{n_{\varphi_{\phi_{r}}}^{i}, i} \longrightarrow \widetilde{Y}_{t}^{i} \text { for } r \longrightarrow \infty \text { in } L^{2}(\Omega) .
$$

But since

$$
X_{t}^{n_{k}^{i}, i} \longrightarrow \widetilde{X}_{t}^{i} \text { for } k \longrightarrow \infty \text { in }(\mathcal{S})^{*}
$$

because of (5.34), we see that

$$
\tilde{Y}_{t}^{i}=\tilde{X}_{t}^{i}
$$


But this leads to the contradiction

$$
\left\|X_{t}^{n_{\varphi_{\phi_{r}}, i}^{i}}-\widetilde{X}_{t}^{i}\right\|_{L^{2}(\Omega)} \geq \varepsilon
$$

This completes the proof.

We are coming to the main result of this section

Theorem 5.11 Assume that the functions $B:[0, T] \times H \longrightarrow H$ and $B^{n}:[0, T] \times H \longrightarrow$ $H, n \geq 1$ satisfy the conditions (5.31) and (5.32). Then there exists a Malliavin differentiable unique mild solution $X_{t}, 0 \leq t \leq T$ to the stochastic differential equation

$$
d X_{t}=A X_{t} d t+B\left(t, X_{t}\right) d t+Q^{1 / 2} d W_{t}, X_{0}=x .
$$

Proof. Let $X_{t}^{n}, n \geq 1$ be the mild solutions associated with the coefficients $B^{n}$ and denote by $X_{t}^{n, i}$ the $i$-th component of $X_{t}^{n}$. Then it follows from Lemma 5.10 that there exists for all $i$ a subsequence $\left(n_{k}^{i}\right)_{k \geq 1}$ which only depends on (a sufficiently small) $T$ and $i$ such that for all $0 \leq t \leq T$

$$
X_{t}^{n_{k}^{i}, i} \longrightarrow X_{t}^{i} \text { in } L^{2}(\Omega) \text { for } k \longrightarrow \infty
$$

for some $X_{t}^{i} \in L^{2}(\Omega), 0 \leq t \leq T, i \geq 1$.

Now let us denote by $\left(\varphi_{n}\right)_{n \geq 1}$ the diagonal sequence of the sequences $\left(n_{k}^{1}\right)_{k \geq 1},\left(n_{k}^{2}\right)_{k \geq 1},\left(n_{k}^{3}\right)_{k \geq 1}, \ldots$

So

$$
X_{t}^{\varphi_{n}, i} \longrightarrow X_{t}^{i} \text { for } n \longrightarrow \infty
$$

in $L^{2}(\Omega)$ for all $t, i$.

We now want to show that

$$
X_{t}^{\varphi_{n}} \longrightarrow X_{t} \text { for } n \longrightarrow \infty
$$

in $L^{2}(\Omega ; H)$ for all $t$, where $X_{t}=\sum_{k \geq 1} X_{t}^{k} e_{k}$. For this purpose choose a $\epsilon>0$. By a weak compactness argument we also see from Lemma 5.8 that

$$
E\left[\left(\sum_{k \geq 1}\left|X_{t}^{k}\right|^{2} \alpha_{k}^{1-\delta}\right)\right]<\infty .
$$

This implies

$$
\begin{aligned}
& \sup _{n \geq 1} \sum_{k \geq m} E\left[\left|X_{t}^{\varphi_{n}, k}-X_{t}^{k}\right|^{2}\right] \\
\leq & \sup _{n \geq 1} E\left[\sum_{k \geq m}\left|X_{t}^{\varphi_{n}, k}-X_{t}^{k}\right| \frac{1}{\alpha_{k}^{(1-\delta) / 2}}\left|X_{t}^{\varphi_{n}, k}-X_{t}^{k}\right| \alpha_{k}^{(1-\delta) / 2}\right] \\
\leq & \sup _{n \geq 1} E\left[\left(\sum_{k \geq m}\left|X_{t}^{\varphi_{n}, k}-X_{t}^{k}\right|^{2} \frac{1}{\alpha_{k}^{1-\delta}}\right)^{1 / 2}\left(\sum_{k \geq m}\left|X_{t}^{\varphi_{n}, k}-X_{t}^{k}\right|^{2} \alpha_{k}^{1-\delta}\right)^{1 / 2}\right] \\
\leq & \sup _{n \geq 1}\left(E\left[\left(\sum_{k \geq m}\left|X_{t}^{\varphi_{n}, k}-X_{t}^{k}\right|^{2} \frac{1}{\alpha_{k}^{1-\delta}}\right)\right]\right)^{1 / 2}\left(E\left[\left(\sum_{k \geq m}\left|X_{t}^{\varphi_{n}, k}-X_{t}^{k}\right|^{2} \alpha_{k}^{1-\delta}\right)\right]\right)^{1 / 2} \\
& C\left(\sum_{k \geq m} \frac{1}{\alpha_{k}^{1-\delta}}\right)^{1 / 2} \\
< & \epsilon
\end{aligned}
$$


for $m \geq m_{0}$. Choosing a $n_{0}$ such that for all $n \geq n_{0}$

$$
\sum_{k \geq 1}^{m_{0}-1} E\left[\left|X_{t}^{\varphi_{n}, k}-X_{t}^{k}\right|^{2}\right]<\epsilon
$$

we find that

$$
E\left[\left\|X_{t}^{\varphi_{n}, k}-X_{t}\right\|^{2}\right]<2 \epsilon
$$

for all $n \geq n_{0}$. So (5.36) holds.

Finally it follows from dominated convergence that (measurability/continuous modification of $(\omega, t) \longmapsto X_{t}(\omega)$ can be shown)

$$
\begin{aligned}
& E\left[\| \int_{0}^{t} e^{(t-s) A}\left(B^{n}\left(s, X_{s}^{\varphi_{n}}\right)-B\left(s, X_{s}\right) d s \|^{2}\right]\right. \\
\leq \quad 2 & \sup _{0 \leq s \leq T}\left\|e^{s A}\right\|^{2}\left(E\left[\int_{0}^{t}\left\|B^{n}\left(s, X_{s}^{\varphi_{n}}\right)-B^{n}\left(s, X_{s}\right)\right\|^{2} d s\right]\right. \\
& \left.+E\left[\int_{0}^{t}\left\|B^{n}\left(s, X_{s}\right)-B\left(s, X_{s}\right)\right\|^{2} d s\right]\right) \\
\leq \quad & 2 \sup _{0 \leq s \leq T}\left\|e^{s A}\right\|^{2} K^{2}\left(\int_{0}^{t}\left(E\left[\left\|X_{s}^{n}-X_{s}\right\|^{2}\right]\right)^{\alpha} d s\right. \\
& \left.+E\left[\int_{0}^{t}\left\|B^{n}\left(s, X_{s}\right)-B\left(s, X_{s}\right)\right\|^{2} d s\right]\right) \\
\longrightarrow & 0 \text { for } n \longrightarrow \infty .
\end{aligned}
$$

From this we see that $X_{t}$ is a mild solution to (5.35). Uniqueness was shown in Section 5.1.

Remark 5.12 Another approach based on the so called $S$-transform to verify $X_{t}$ as a unique solution to SDE's is discussed in [16], [15].

Example 5.13 Consider the equation

$$
d X(t, \xi)=(\Delta X(t, \xi)+B(t, X(t, \cdot))(\xi)) d t+\sigma(-\Delta)^{-\gamma / 2} d W(t, \xi)
$$

for $t \geq 0$ and $\xi \in[0,2 \pi]$, with periodic boundary conditions. In this case we have $H=$ $L^{2}(0,2 \pi)$ and $A=\Delta$. We let $Q=(-\Delta)^{-\gamma}$ with $0<\gamma<\frac{1}{3}, \theta=\frac{1}{2}$ in Section 5.1.1 and $M=(-\Delta)^{\lambda}$ for a sufficiently small $\lambda>0$, then the conditions of Section 5.1 .1 and the conditions of Theorem 5.11.

Proof. Let us show that (5.19) holds. We have

$$
\begin{aligned}
& \left\|e^{\left(t-u_{1}\right) A} Q^{1 / 2}-e^{\left(t-u_{2}\right) A} Q^{1 / 2}\right\|_{E}^{2} \\
= & \sum_{n=1}^{\infty}\left(e^{-\left(t-u_{1}\right) \alpha_{n}} \alpha_{n}^{-\gamma / 2}-e^{-\left(t-u_{2}\right) \alpha_{n}} \alpha_{n}^{-\gamma / 2}\right)^{2} \alpha_{n}^{2 \lambda} \\
= & \sum_{n=1}^{\infty}\left(1-e^{-\left(u_{1}-u_{2}\right) \alpha_{n}}\right)^{2} e^{-2\left(t-u_{1}\right) \alpha_{n}} \alpha_{n}^{2 \lambda-\gamma} .
\end{aligned}
$$


Now, for $\epsilon, \nu \in(0,1)$ we can find positive constants $C_{\epsilon}$ and $C_{\nu}$ such that

$$
\left(1-e^{-a}\right) \leq C_{\epsilon} a^{\epsilon} \quad \text { and } \quad e^{-2 a} \leq C_{\nu} a^{-\nu}
$$

for every $a \geq 0$. Thus, the above is bounded by

$$
\begin{gathered}
C_{\epsilon}\left|u_{1}-u_{2}\right|^{2 \epsilon} \sum_{n=1}^{\infty} \alpha_{n}^{2 \epsilon+2 \lambda-\gamma} e^{-2\left(t-u_{1}\right) \alpha_{n}} . \\
\leq C_{\epsilon} C_{\nu}\left|u_{1}-u_{2}\right|^{2 \epsilon}\left|t-u_{1}\right|^{-\nu} \sum_{n=1}^{\infty} \alpha_{n}^{2 \epsilon+2 \lambda-\gamma+\nu} .
\end{gathered}
$$

Rewriting this condition in the Fourier basis on the interval $[0,2 \pi]$, we get the condition

$$
\sum_{k \in \mathbb{Z}} k^{4 \epsilon+4 \lambda-2 \gamma+2 \nu}<\infty .
$$

Let $\nu=1-\delta$ and choose $\epsilon$ and $\lambda$ small to get (5.19). Inequality (5.18) is proved similary.

To see (5.20) we write

$$
\int_{H}\left\|e^{\left(u_{1}-u_{2}\right) A} y-y\right\|^{2} N_{Q_{s}}(d y)=E\left[\left\|e^{\left(u_{1}-u_{2}\right) A} \sqrt{Q_{s}} W_{1}-\sqrt{Q_{s}} W_{1}\right\|^{2}\right]
$$

where $W=\left(W_{t}\right)_{t \geq 0}$ is a cylindrical Brownian motion, $W_{t}=\sum_{n=1}^{\infty} W_{t}^{n} e_{n}$. We get

$$
\left(e^{\left(u_{1}-u_{2}\right) A} \sqrt{Q_{s}}-\sqrt{Q_{s}}\right) W_{1}=\sum_{n=1}^{\infty}\left(e^{-\left(u_{1}-u_{2}\right) \alpha_{n}}-1\right)\left(\int_{0}^{s} q_{n} e^{-2 u \alpha_{n}} d u\right)^{1 / 2} W_{1}^{n},
$$

so that

$$
\begin{aligned}
E\left[\left\|e^{\left(u_{1}-u_{2}\right) A} \sqrt{Q_{s}} W_{1}-\sqrt{Q_{s}} W_{1}\right\|^{2}\right] & =\sum_{n=1}^{\infty}\left(e^{-\left(u_{1}-u_{2}\right) \alpha_{n}}-1\right)^{2} \int_{0}^{s} q_{n} e^{-2 u \alpha_{n}} d u \\
& \leq C_{\epsilon} \sum_{n=1}^{\infty}\left(\left(u_{1}-u_{2}\right) \alpha\right)^{2 \epsilon} \frac{q_{n}\left(1-e^{-2 s \alpha_{n}}\right)}{2 \alpha_{n}} \\
& \leq C_{\epsilon}\left|u_{1}-u_{2}\right|^{2 \epsilon} \sum_{n=1}^{\infty} \alpha_{n}^{2 \epsilon-1-\gamma} .
\end{aligned}
$$

As before we rewrite this in the Fourier basis we get the condition

$$
\sum_{k \in \mathbb{Z}} k^{4 \epsilon-2-2 \gamma}<\infty
$$

which is satisfied for small $\epsilon$.

Finally we show that we have $\int_{0}^{T}\left\|\Lambda_{t}\right\|^{1+\theta} d t<\infty$ when $\theta=\frac{1}{2}$. We have

$$
\begin{aligned}
\left\|\Lambda_{t} x\right\|^{2} & =\left\|A^{(1+\gamma) / 2}\left(I-e^{2 t A}\right)^{-1 / 2} e^{t A} x\right\|^{2} \\
& =t^{-(1+\gamma)} \sum_{n=1}^{\infty}\left(t \alpha_{n}\right)^{1+\gamma}\left(1-e^{-2 t \alpha_{n}}\right)^{-1} e^{-2 t \alpha_{n}} .
\end{aligned}
$$


The mapping $s \mapsto \frac{s^{1+\gamma}}{e^{2 s}-1}$ is bounded on $(0, \infty)$, so that we get

$$
\left\|\Lambda_{t}\right\| \leq C t^{-(1+\gamma) / 2}
$$

and thus we get that $t \mapsto\left\|\Lambda_{t}\right\|^{3 / 2}$ is integrable on any interval $[0, T]$.

\subsection{Appendix}

The following result which is based on Malliavin calculus and which is essentially due to [4] provides a compactness criterion for subsets of $L^{2}(\Omega)$ of square integrable functionals of a cylindrical Wiener process $W_{t}, 0 \leq t \leq 1$ on the Hilbert space $H$. See e.g. [17], [13] or [7] for more information about Malliavin calculus.

Theorem 5.14 Assume that $L$ is a self-adjoint compact operator on $H^{*}$ with dense image. Denote by $D X \in L^{2}\left(\Omega ; L^{2}([0,1]) \otimes H^{*}\right)$ the Malliavin derivative of a square integrable $X$ in the domain of $D$ and by $\mathbf{D}_{1,2}$ the space of such functionals. Then for $0<\beta<1 / 2$ and $c>0$ the set

$$
\begin{aligned}
\mathcal{G}= & \left\{G \in \mathbf{D}_{1,2}:\|G\|_{L^{2}(\Omega)}+\left(\int_{0}^{1}\left\|L^{-1} D_{u} G\right\|_{L^{2}(\Omega)} d u\right)^{1 / 2}\right. \\
& \left.+\left(\int_{0}^{1} \int_{0}^{1} \frac{\left\|L^{-1}\left(D_{u_{1}} G-D_{u_{2}} G\right)\right\|_{L^{2}(\Omega)}^{2}}{\left|u_{1}-u_{2}\right|^{1+2 \beta}} d u_{1} d u_{2}\right)^{1 / 2} \leq c\right\}
\end{aligned}
$$

is relatively compact in $L^{2}(\Omega)$.

Remark 5.15 Denote by $J: H^{*} \longrightarrow H$ the standard isometric isomorphism for Hilbert spaces $H$. Then an example of $L$ which satisfies the conditions of Theorem 5.14 is given by

$$
L a:=\langle M J(a), \cdot\rangle, a \in H^{*},
$$

where $M=A^{\tau}$ for some sufficiently small $\tau>0$, where $A$ is the densely defined operator in Section 1. 


\section{References}

[1] Beesack, P. R.: Comparison theorems and integral inequalities. Proc. Amer. Math. Soc., 20, 61-66 (1969).

[2] Da Prato, G., Flandoli, F.: Pathwise uniqueness for a class of SDE in Hilbert spaces and applications. Journal of Functional Analysis, 259 (2010) 243-267.

[3] Da Prato, G., Flandoli, F., Priola, E., Röckner, M.: Strong uniqueness for stochastic evolution equations in Hilbert spaces with unbounded measurable drift. Preprint: arXiv:1109.0363

[4] Da Prato, G., Malliavin, P., Nualart, D.: Compact families of Wiener functionals. C.R. Acad. Sci. Paris, t. 315, Série I, p. 1287-1291 (1992).

[5] Da Prato, G., Zabczyk, J.: Stochastic equations in infinite dimensions, Encyclopedia of Mathematics and its Applications, 44, Cambridge University Press, Cambridge, 1992.

[6] Da Prato, G., Zabczyk, J.: Second order partial differential equations in Hilbert spaces, London Mathematical Society Lecture Note Series, 293, Cambridge University Press, Cambridge, 2002.

[7] Di Nunno, G., Øksendal, B., Proske, F.: Malliavin Calculus for Lévy Processes with Applications to Finance. Universitext, Springer (2009).

[8] Flandoli, F.: Random Perturbation of PDE's and Fluid Dynamic Models. LNM 2015, Springer (2011).

[9] Gyöngy, I., Pardoux, E.: On quasi-linear stochastic partial differential equations. Probability Theory and Related Fields 94. 413-425 (1993).

[10] Hida, T., Kuo, H.-S., Potthoff, J., Streit, L.: White Noise- An Infinite Dimensional Calculus. Kluwer (1993).

[11] Krylov, N.V.: Lectures on Elliptic and Parabolic Equations in Hölder Spaces. Graduate Studies in Mathematics. Vol 12. American Mathematical Society (1997).

[12] Lassalle, R.: Causal Transference Plans and Their Monge-Kantorovich Problems. Preprint arXiv: 1303.6925

[13] Malliavin , P.: Stochastic Analysis. Grundlehren der Mathematischen Wissenschaften, Vol. 313, Springer-Verlag, Berlin (1997).

[14] Mitoma, I.: Tightness of probabilities on $C\left([0,1], S^{\prime}\right)$ and $D\left([0,1] ; S^{\prime}\right)$. Annals of Probability, 11(4), p. 989-999 (1983).

[15] Menoukeu-Pamen, O., Meyer-Brandis, T., Nilssen, T., Proske, F., Zhang, T.: A variational approach to the construction and Malliavin differentiability of strong solutions of SDE's. Mathematische Annalen, Vol. 357 Issue 2, pp 761-799, (2013).

[16] Meyer-Brandis, T., Proske, F.:Construction of strong solutions of SDE's via Malliavin calculus. J. of Funct. Anal., 258, 3922-3953 (2010).

[17] Nualart, D.: The Malliavin Calculus and Related Topics. Springer (1995). 
